\title{
O Primeiro Espaço de Banach Hereditariamente Indecomponível
}

\author{
Cintia Cristina da Silva
}

\author{
DISSERTAÇÃO APRESENTADA \\ AO \\ INSTITUTO DE MATEMÁTICA E ESTATÍSTICA \\ DA \\ UNIVERSIDADE DE SÃO PAULO \\ PARA \\ OBTENÇÃO DO GRAU DE MESTRE \\ EM \\ MATEMÁTICA PURA
Área de Concentração : Análise Matemática
Orientador : : Prof. Dr. Elói Medina Galego

Durante a elaboração deste trabalho a autora recebeu auxilio financeiro parcial do CNPq.

São Paulo, setembro de 2000 


\title{
O Primeiro Espaço de Banach Hereditariamente Indecomponível
}

\author{
Este exemplar corresponde à redação \\ final da dissertação devidamente corrigida \\ e defendida por Cintia Cristina da Silva \\ e aprovada pela comissão julgadora.
}

Banca examinadora:

- Prof. Dr. Elói Medina Galego (orientador) - IME-USP

- Prof. Dr. Chaim Samuel Hönig - IME-USP

- Prof. Dr. Raymundo Luiz de Alencar - IMECC-UNICAMP

São Paulo, setembro de 2000 . 
"Bem aventurado o homem que acha sabedoria,

e o homem que adquire conhecimento."

A minha mãe e irmãs, Kátia e Andréa. 


\title{
Resumo
}

O objetivo deste trabalho é estudar o primeiro espaço de Banach hereditariamente indecomponível construído por W. T. Gowers e B. Maurey.

Os espaços hereditariamente indecomponíveis tem sido usado para resolverem muitos problemas, que ficaram em aberto por muitos anos, tais como o problema do hiperlano de Banach.

\begin{abstract}
The purpose of this work is to present the first Banach space hereditarily indecomposable constructed by Gowers, W. T. and Maurey, B.

The hereditarily indecomposable spaces has been used to solve several long-standing problems like the Banach's hyperplane problem.
\end{abstract}




\section{Agradecimentos}

- A Deus, por ter tornado possível o desejo do meu coração e ter me dado forças nos momentos que mais precisei.

- Ao Professor Elói Medina Galego, pela sugestão do tema e pela paciente orientação.

- A minha mãe Lourdes e irmàs Kátia e Andréa, pelo amor incondicional e todo apoio.

$\diamond$ A todos professores e colegas de cursos que, diretamente ou indiretamente, contribuiram para realização deste trabalho, em especial à Professora Zara Issa Abud por todo apoio e motivação.

- Aos Professores W. T. Gowers, Thomas Schlumprecht e Manuel Gonzalez, pelos e-mail e materiais que deles recebemos esclarecendo várias dúvidas ao longo dessa dissertação.

$\diamond$ A todos meus amigos, por estarem ao meu lado em todos os momentos.

- A CNPq pelo apoio financeiro no início do programa. 


\section{Sumário}

$\begin{array}{ll}\text { Notações } & 8\end{array}$

$\begin{array}{ll}\text { Introdução } & 10\end{array}$

$\begin{array}{lll}0 & \text { Preliminares } & 12\end{array}$

1 Espaços Precursores 18

1.1 O espaço de Schreier . . . . . . . . . . . . . . . . . . 19

$1.2 \mathrm{O}$ espaço de Baernstein . . . . . . . . . . . . . . . . . . 24

1.3 O espaço de Tsirelson . . . . . . . . . . . . . . . . . . . . . . . . . 29

2 Funções 40

3 O espaço de Thomas Schlumprecht $\quad 52$

3.1 Conjuntos assintóticos . . . . . . . . . . . . . . . . 52

3.2 O espaço de Schlumprecht . . . . . . . . . . . . . . 57

4 O espaço de Gowers e Maurey $\quad 83$

5 Operadores sobre espaços hereditariamente indecomponíveis $\quad 114$ 
Referências Bibliográficas 


\section{Notações}

Sejam $X$ e $Y$ espaços de Banach, dizemos que $X$ tem uma cópia isomorfa de $Y$, denotaremos por $Y \hookrightarrow X$, se existe um operador linear contínuo injetor $\varphi: Y \rightarrow X$ com a imagem fechada e com inversa contínua.

Seja $X^{\prime}$ um espaço de Banach sobre $\mathbb{R}$ ou $\mathbb{C}$, indicaremos por $X^{*}$ o conjunto de todas as aplicações lineares contínuas de $X$ (o dual topológico de $X$ ).

Seja $X$ um espaço de Banach, indicaremos por $B(X)=\{x \in X:\|x\| \leq 1\}$ a bola fechada de centro em zero e raio um em $X$.

Sejam $X$ e $Y$ espaços normados, $L(X, Y)$ representará o conjunto das transformações lineares contínuas de $X$ em $Y$.

Seja $X^{\prime}$ um espaço de Banach, por $\operatorname{dim}\left(X^{\prime}\right)$ denotaremos a dimensão do espaço de Banach $X$, que pode ser finita ou infinita.

Sejam $X$ e $Y$ espaços de Banach e $T \in L(X, Y)$, por $K$ er $(T)$ denotaremos o núcleo de $T$ e por $\operatorname{Im}(T)$ denotaremos a imagem de $T$.

Por $c_{0}(\mathbb{N})$ denotaremos o espaço de Banach das sequências convergentes a zero, com a norma do supremo.

Por $l_{p}(\mathbb{N})$ denotaremos o espaço de Banach das sequências p-somáveis com a norma $\left\|\left(a_{n}\right)_{n}\right\|_{l_{p}}=\left[\sum_{i=1}^{\infty}\left|a_{n}\right|^{p}\right]^{1 / p}$.

Por $c_{00}$ denotaremos o espaço vetorial das sequências de escalares com apenas um número finito de coordenadas não nulas e por $\left(e_{n}\right)_{n}$ denotaremos a base vetorial unitária desse espaço. 
Sejam $E \subset \mathbb{N}$ e $\mathcal{S}$ o conjunto de todas as sequências de escalares, usaremos a letra $E$ para denotar a projeção de $\mathcal{S}$ em $\mathcal{S}$ definida por $E\left(\sum_{i=1}^{\infty} a_{i} e_{i}\right)=\sum_{i \in E} a_{i} e_{i}$.

Se $E, F \subset \mathbb{N}$ então escreveremos $E \leq F$ para indicar que $\max E \leq \min F$ e $n \leq E$ para indicar que $\{n\} \leq E$.

Para nós, um intervalo de inteiros será um subconjunto dos números naturais da forma $\{a, a+1, \ldots, b\}$ para algum $a, b \in \mathbb{N}$.

Por $\operatorname{supp}(x)$ denotaremos o suporte de um vetor $x=\sum_{i=1}^{\infty} a_{i} e_{i} \in c_{00}$, isto é, $\operatorname{supp}(x)=$ $\left\{i \in \mathbb{N}: a_{i} \neq 0\right\}$ e por $\operatorname{ran}(x)$ denotaremos o menor intervalo em $\mathbb{N}$ contendo o seu suporte.

Finalmente, escreveremos $x<y$ quando $\operatorname{ran}(x)<\operatorname{ran}(y)$. Se $x_{1}<\ldots<x_{n}$ diremos que $x_{1}, \ldots, x_{n}$ são sucessivos.

Escreveremos $a \vee b$ para indicar $\max \{a, b\}$, onde $a, b \in \mathbb{R}$.

Usaremos HI como abreviaçào de hereditariamente indecomponível.

O símbolo a indica o término de uma demonstração. 


\section{Introdução}

O principal objetivo desta dissertação é apresentar em detalhes o primeiro espaço de Banach $X$ (real e complexo) hereditariamente indecomponível, isto é. nenhum subespaço fechado de $X$ pode ser decomposto como soma direta de dois subespaços fechados de dimensão infinita.

Esse espaço foi construído em 1993 por W. T. Gowers e B. Maurey e foi obtido para solucionar o famoso problema da sequência básica incondicional, ou seja, $X$ é um exemplo de espaço de Banach que não possui nenhuma sequência básica incondicional.

Nós usaremos a teoria espectral para mostrar que todo operador linear contínuo definido num espaço hereditariamente indecomponivel complexo. é da forma $\lambda I+S$ onde $\lambda \in \mathbb{C}, I$ é o operador identidade nesse espaço e $S$ é estritamente singular.

Como consequência disto, $X$ não é isomorfo a nenhum de seus subespaços próprios, em particular, $X$ não é isomorfo a nenhum de seus hiperplanos.

Também mostraremos que os hiperplanos da versão real de $X$ tem a mesma propriedade e portanto resolve negativamente o famoso problema do hiperplano proposto por Banach.

O espaço $X$ e seus subespaços tem sido usado para a construção de outros contraexemplos, veja por exemplo [8] e [10].

Os espaços hereditariamente indecomponiveis tornaram-se importantes para a geometria de espaços de Banach devido ao teorema de dicotomia de Gowers, [13], "Todo espaço de Banach de dimensão infinita contém um subespaço com base incondicional ou um subespaço hereditariamente indecomponivel".

Finalmente, observamos que outros espaços hereditariamente indecomponíveis foram 
construídos, veja a bibliografia de [12], no entanto, ainda não se sabe se existem espaços de Banach hereditariamente indecomponiveis não separáveis. 


\section{Capítulo 0}

\section{Preliminares}

Neste capítulo. apresentaremos algumas definições e resultados sobre a teoria dos espaços de Banach que serão utilizados nos capítulos seguintes.

Definição 0.1 Uma sequência $\left(x_{n}\right)_{n}$ num espaço de Banach $E$ é uma base de Schauder de $E$ se, e somente se, para cada $x \in E$, existe uma única sequência de escalares $\left(a_{n}\right)_{n}$ tal que $x=\sum_{n=1}^{\infty} a_{n} x_{n}$.

Definição 0.2 Uma sequência $\left(x_{n}\right)_{n}$ que é uma base de Schauder do subespaço fechado gerado por $\left(x_{n}\right)_{n}$, isto é $\left[\left(x_{n}\right)_{n}\right]$, é chamada de sequência básica.

Teorema 0.3 (6, pág 47) Seja $\left(x_{n}\right)_{n}$ uma sequência de elementos não nulos no espaço de Banach E. Então $\left(x_{n}\right)_{n}$ é uma sequência básica se, e somente se, existe um número real positivo $k$ tal que para qualquer sequência de escalares $\left(a_{n}\right)_{n}$ e inteiros $m<n$, tem-se $\left\|\sum_{i=1}^{m} a_{i} x_{i}\right\| \leq k\left\|\sum_{i=1}^{n} a_{i} x_{i}\right\|$.

Definição 0.4 Uima base de Schauder $\left(x_{n}\right)_{n}$ é incondicional se sempre que a série $\sum_{n=1}^{\infty} a_{n} x_{n}$ converge, ela converge incondicionalmente.

Teorema 0.5 (1, pág 88-91) Para uma base de Schauder $\left(x_{n}\right)_{n}$, as seguintes propriedades são equivalentes: 
a) $\left(x_{n}\right)_{n}$ é uma base incondicional.

b) Existe uma constante $C \geq 1$ tal que se $A$ e $B$ são subconjuntos finitos dos naturais com $A \subset B$, entâo para qualquer sequência $\left(a_{n}\right)_{n}$ de escalares $\left\|\sum_{n \in A} a_{n} x_{n}\right\| \leq C\left\|\sum_{n \in B} a_{n} x_{n}\right\|$. c) Existe uma constante $C \geq 1$ tal que, para toda sequência de escalares $\left(a_{n}\right)_{n}$ e toda sequência de escalares $\left(\epsilon_{n}\right)_{n}$ de módulo no máximo 1 , nós temos a inequação $\left\|\sum_{n=1}^{\infty} \epsilon_{n} a_{n} x_{n}\right\| \leq$ $C\left\|\sum_{n=1}^{\infty} a_{n} x_{n}\right\|$

Uma base satisfazendo o ítem b) ou o ítem c) será chamada de C-incondicional.

Definição 0.6 Seja E um espaço de Banach com base de Schauder $\left(x_{n}\right)_{n}$. Para todo inteiro $n$ o funcional linear contínuo $x_{n}^{*}$ definido por $x_{n}^{*}\left(\sum_{i=1}^{\infty} a_{i} x_{i}\right)=a_{n}$ é chamado de funcional biortogonal.

Definição 0.7 Sejam $\left(x_{n}\right)_{n}$ uma sequência básica em $E,\left(p_{n}\right)_{n}$ uma sequência estritamente crescente de inteiros positivos e $\left(t_{n}\right)_{n}$ uma sequência de números reais. Uma sequência $\left(z_{n}\right)_{n}$ dada por $z_{n}=\sum_{i=p_{n}+1}^{p_{n+1}} t_{i} x_{i}$. com $z_{n} \neq 0, \forall n \in \mathbb{N}$ é chamada de base de blocos de $\left(x_{n}\right)_{n}$.

Definição 0.8 Duas bases de Schauder $\left(x_{n}\right)_{n}$ e $\left(y_{n}\right)_{n}$ de espaços de Banach E e F respectivamente são ditas equivalentes se $\sum_{n=1}^{\infty} a_{n} x_{n}$ converge se, e somente se, $\sum_{n=1}^{\infty} a_{n} y_{n}$ também
converge.

Teorema 0.9 (6, pág 53) Seja $\left(x_{n}\right)_{n}$ uma base do espaço de Banach E com funcionais biortogonais $\left(x_{n}^{*}\right)_{n}$. Se uma sequência $\left(y_{n}\right)_{n}$ em E satisfaz

a) $\inf _{n}\left\|y_{n}\right\|=\epsilon>0$.

b) $x_{i}^{*}\left(y_{n}\right) \stackrel{n \rightarrow+\infty}{\rightarrow} 0, \forall i \in \mathbb{N}$.

Então existe uma subsequência $\left(y_{p_{n}}\right)_{n}$ que é uma sequência básica e essa sequência é equivalente a uma base de bloco de $\left(x_{n}\right)_{n}$. 
Teorema 0.10 (20, pág 6) Seja E um espaço de Banach com base de Schauder $\left(x_{n}\right)_{n}$. Seja $Y$ um subespaço fechado de dimensão infinita de $E$. Então existe um subespaço $Z$ de $Y$ que tem uma base que é equivalente a uma base de bloco de $\left(x_{n}\right)_{n}$.

Definição 0.11 Seja E um espaço de Banach e $\left(x_{n}\right)_{n}$ uma base de E, a) $\left(x_{n}\right)_{n}$ é "shrinking" se para todo funcional linear contínuo $x^{*}$ e para todo $\epsilon>0$ existe $n \in \mathbb{N}$ tal que a norma de $x^{*}$ restrito ao espaço gerado por $x_{n}, x_{n+1}, \ldots$ é no máximo $\epsilon$. b) $\left(x_{n}\right)_{n}$ é limitadamente completa se dada qualquer sequência de escalares $\left(a_{n}\right)_{n}$ para a qual a soma parcial $\sum_{n=1}^{N} a_{n} x_{n}$ é limitada então a série $\sum_{n=1}^{\infty} a_{n} x_{n}$ converge.

Teorema 0.12 (20, pág 22) Seja E um espaço de Banach com base incondicional $\left(x_{n}\right)_{n}$. Então as seguintes condiçôes são equivalentes:

a) A base é limitadamente completa.

b) E não tem subespaço isomorfo a $c_{0}(\mathbb{N})$.

Teorema 0.13 (18, pág 637) Se E é um espaço de Banach com base incondicional, então $E$ é reflexivo a menos que ele contenha um subespaço isomorfo a $c_{0}(\mathbb{N})$ ou um subespaço isomorfo a $l_{1}(\mathbb{N})$.

Teorema 0.14 (3, pág 2) Sejam E um espaço de Banach com base de Schauder $\left(x_{n}\right)_{n}$ e $\delta>0$. Se $E$ contém um subespaço isomorfo a $l_{1}(\mathbb{N})$, então existe uma sequência $\left(u_{n}\right)_{n}$ normalizada de blocos de $\left(x_{n}\right)_{n}$ tal que $(1-\delta) \sum_{i=1}^{\infty}\left|a_{i}\right|<\left\|\sum_{i=1}^{\infty} a_{i} u_{i}\right\| \leq \sum_{i=1}^{\infty}\left|a_{i}\right|$ para toda sequência finita de escalares $\left(a_{i}\right)_{i}$ que não são todos nulos.

Definição 0.15 Sejam $E$ um espaço de Banach e $\left(x_{n}\right)_{n} \subset E$ uma sequência então:

a) $\left(x_{n}\right)_{n}$ é fracamente de Cauchy se a sequência $x^{*}\left(x_{n}\right)$ é convergente para cada $x^{*} \in E^{*}$.

b) $\left(x_{n}\right)_{n}$ é fracamente nula se $x^{*}\left(x_{n}\right) \rightarrow 0$ para cada $x^{*} \in E^{*}$.

Teorema 0.16 (4, pág 201) Sejam E um espaço de Banach e $\left(x_{n}\right)_{n}$ uma sequência limitada em E. Então existe uma subsequência $\left(x_{n_{k}}\right)_{k}$ de $\left(x_{n}\right)_{n}$ que é uma sequência fracamente de Cauchy ou existe uma subsequência $\left(x_{n_{k}}\right)_{k}$ de $\left(x_{n}\right)_{n}$ que é equivalente á base canônica de $l_{1}(\mathbb{N})$. 
Teorema 0.17 (Teorema da Aplicação Aberta, 15, pág 245) Seja fuma aplicação linear contínua de um espaço de Banach $E$ sobre um espaço de Banach $F$ então para todo conjunto aberto $l i \subset E, f(U)$ é aberto em $F$.

Teorema 0.18 (Teorema do gráfico fechado, 15, pág 251) Sejam E e $F$ espaços de Banach e $f: E \rightarrow F$ uma aplicação linear. $f$ é contínua se, e somente se, o seu gráfico é fechado.

Definição 0.19 Um subespaço $M$ de um espaço vetorial $V$ tem codimensão finita em $V$ se a dimensão do quociente de $V / M$ é finita, ou seja. não existe uma quantidade infinita de vetores linearmente independente fora de $M$.

Como estaremos trabalhando com espaços de Banach. todos os nossos subespaços de codimensão finita serão fechados.

Teorema 0.20 (7, pág 103) Seja E um espaço de Banach e $M$ um subespaço de $E$ de codimensão finita. Então para qualquer subespaço $Y$ de E: existe $Z$ um subespaço de dimensão finita tal que $Y=M \cap Y \oplus Z$.

Definição 0.21 Sejam $E$ e $F$ dois espaços de Banach e $T \in L(E, F)$, dizemos que $T$ é compacto se para cada sequência limitada $\left(x_{n}\right)_{n}$ em E. $T\left(x_{n}\right)_{n}$ admite uma subsequência convergente em norma.

Teorema 0.22 (20, pág 76) Sejam $E$ e $F$ espaços de Banach de dimensão infinita. Assuma que $T: E \rightarrow F$ é um operador tal que a restrição de $T$ a qualquer subespaço de $E$ de codimensão finita não é um isomorfismo. Então, para todo $\epsilon>0$ existe um subespaço fechado de dimensão infinita $Z$ de $E$ tal que $\left.T\right|_{Z}$ é compacto e $\left\|\left.T\right|_{Z}\right\| \leq \epsilon$.

Definição 0.23 Seja $T: E \rightarrow F$ um operador linear continuo para o qual $T(E)$ é fechada. Se a dimensão do núcleo de $T$ é finita ou a dimensão do quociente de $F / T(E)$ é finita. então definimos o indice de $T$. denotaremos $i(T)$. como sendo a diferença entre a dimensão do núcleo de $T$ e a dimensão do quociente de $F / T(E)$. 
Definição 0.24 Um operador $T: E \rightarrow E$ é estritamente singular quando a restrição de $T$ a qualquer subespaço fechado de $E$ de dimensão infinita não é um isomorfismo.

Teorema 0.25 (20, pág 78) Seja $T: E \rightarrow F$ um operador para o qual $i(T)$ está definido. Existe um número $\lambda(T)>0$ tal que se $S: E \rightarrow F$ satisfaz $\|S\|<\lambda(T)$ então $i(T+S)=i(T)$.

Teorema 0.26 (20, pág 79) Seja $T: E \rightarrow F$ um operador com imagem fechada para o qual a dimensão do núcleo de $T$ é finita. Seja $S: E \rightarrow F$ um operador estritamente singular. Então $i(T+S)=i(T)$.

Definição 0.27 resolvente $\rho(T)$ de $T \in L(E)$ é o conjunto de todos os $\lambda$ tais que a imagem de $\lambda-T$ é densa em $E$ e $\lambda-T$ tem inversa contínua. O espectro de $T e ́ o$ conjunto $S_{p}(T)$ complementar do resolvente.

Definição $0.28 \lambda$ é um autovalor aproximado de $T \in L(E)$ se $\forall \epsilon>0, \exists x,\|x\|=1$ tal que $\|\lambda x-T(x)\|<\epsilon$.

Teorema 0.29 (24, pág 282) O conjunto dos autovalores aproximados de $T$ é um subconjunto fechado do espectro e inclui todos os pontos da fronteira do espectro.

Se $T \in L(E)$, onde $E$ é um espaço de Banach complexo, então o conjunto dos autovalores aproximados de $T$ é não vazio.

Teorema 0.30 (24, pág 193) Sejam E e F espaços de Banach e $G$ o conjunto de todos os elememtos inversiveis de $L(E, F)$. Se $A \in G, B \in L(E, F)$ e $\|A-B\|<\frac{1}{\left\|A^{-1}\right\|}$, então $B \in G$.

Definição 0.31 Uma função $f$ real é concava em um intervalo, se para todo a e b deste intervalo, o segmento retilíneo que une $(a, f(a))$ com $(b, f(b))$ fica por baixo do gráfico de $f$. Dizemos que $f$ é convexa se $-f$ é concava.

Observação 0.32 É conhecido do cálculo diferencial que se $f$ tem derivada até segunda ordem positiva num intervalo $(a, b)$, então $f$ é convexa nesse intervalo. Também é um 
exercício de cálculo a Desigualdade de Jensen, isto é. seja $G$ uma função concava $e$ $\sum_{i=1}^{n} \lambda_{i}=1$, então $G\left(\sum_{i=1}^{n} \lambda_{i} x_{i}\right) \geq \sum_{i=1}^{n} \lambda_{i} G\left(x_{i}\right)$, veja [23, pág 292].

Observação 0.33 S'egue imediatamente da definição acima que são equivalentes:

a) $f$ é convexa.

b) O epigráfico de $f$, isto é, $A_{f}=\left\{(x . y): x \in D_{f}, f(x) \leq y\right\}$ é convexo.

c) $\forall\left(x_{1}, y_{1}\right),\left(x_{2}, y_{2}\right) \in D_{f} \times \mathbb{R}$ tais que $f\left(x_{1}\right)<y_{1}$ e $f\left(x_{2}\right)<y_{2}$, tem-se que $f\left(\lambda x_{1}+(1-\lambda) x_{2}\right) \leq \lambda y_{1}+(1-\lambda) y_{2}$. 


\section{Capítulo 1}

\section{Espaços Precursores}

Como já foi dito na introdução, o nosso principal objetivo é estudar o espaço de Banach (reflexivo) de Gowers e Maurey obtido pelo completado de um espaço normado em $c_{00}$. Esse espaço não contem nenhuma sequência básica incondicional, em particular, ele não possue nenhum subespaço isomorfo a $c_{0}(\mathbb{N})$, ou $l_{p}(\mathbb{N}), 1 \leq p<+\infty$, pois as bases canônicas desses espaços são incondicionais.

Neste capítulo apresentaremos alguns espaços de Banach $E$ obtidos também pelo completado de espaços normados em $c_{00}$. Eles podem ser considerados precursores do espaço de Thomas Schlumprecht (veja capítulo 3) e do espaço de Gowers e Maurey (veja capítulo 4) pela maneira com que suas normas são definidas.

Motivados pelo Teorema de James, veja Teorema 0.13, estaremos interessados, neste capítulo, em responder as seguintes perguntas:

1. E contém uma base incondicional ?

2. $E$ possue subespaços isomorfos a $c_{0}(\mathbb{N})$ ?

3. $E$ possue subespaços isomorfos a $l_{1}(\mathbb{N})$ ?

4. $E$ é reflexivo?

Mais concretamente, inicialmente mostraremos que o espaço de Schreier (S), contém uma base 1-incondicional, não possue subespaço isomorfo a $l_{1}(\mathbb{N})$. mas possue um subespaço isomorfo a $c_{0}(\mathbb{N})$, veja Proposição 1.3. Teorema 1.4 e Teorema 1.6.

A seguir provaremos que o espaço de Baernstein (B) contém uma base 1-incondicional, 
mas não contém subespaço isomorfo a $l_{1}(\mathbb{N})$ ou $c_{0}(\mathbb{N})$ e é reflexivo, veja Proposição $1 . \bar{\imath}$ e Teorema 1.10 .

Finalmente, apresentaremos o espaço de Tsirelson ( $\mathrm{T}$ ), que foi construido para mostrar a existência de um espaço de Banach reflexivo que não possue nem subespaço isomorfo a $c_{0}(\mathbb{N})$ ou $l_{p}(\mathbb{N}), 1 \leq p<+\infty$, veja Teorema 1.12 , Teorema 1.13 e Observação 1.14.

A construção do espaço de Tsirelson que nós usamos aqui é devida a T. Fiegel e W. B. Jonhson. Na verdade, ele é o dual do exemplo original de B. S. Tsirelson.

As Proposições e Teoremas estudados neste capítulo podem ser encontrados em [3].

\subsection{O espaço de Schreier}

Definição 1.1 Todo conjunto finito ordenado $E=\left\{n_{1}<n_{2}<\ldots<n_{k}\right\}$ de números naturais é chamado admissivel se $k \leq n_{1}$, ou seja, o número de elementos do conjunto é menor ou igual ao primeiro elemento do conjunto.

Observação 1.2 Todo subconjunto de um conjunto admissivel é admissivel.

De fato, seja $E$ um conjunto admissivel e $F$ um subconjunto de $E$ então $E=\left\{n_{1}<\right.$ $\left.n_{2}<\ldots<n_{k}\right\}$ onde $k \leq n_{1}$ e $F=\left\{n_{p}<\ldots<n_{q}\right\}$. Temos que $q \leq k \leq n_{1} \leq n_{p}$, portanto $q \leq n_{p}$ e $\operatorname{assim} F$ é admissivel.

Denotaremos por $\mathcal{N}$ a classe de todos os conjuntos admissiveis.

O espaço de Schreier, denotado por S, é definido como sendo o completado de $c_{00}$ com a norma $\|x\|_{S}=\sup _{E \in \mathcal{N}} \sum_{k \in E}\left|x_{k}\right|$, onde $x=\left(x_{k}\right)_{k}$.

Notemos que $\|x\|_{S}$ é norma em $c_{00}$, de fato sejam $x=\left(x_{k}\right)_{k}$ e $y=\left(y_{k}\right)_{k}$ elementos de $c_{00}$

$$
\begin{aligned}
& \text { 1) claramente }\|x\|_{S} \geq 0 . \\
& \|x\|_{S}=0 \Longleftrightarrow \sup _{E \in X^{\prime}} \sum_{k \in E}\left|x_{k}\right|=0 \Longleftrightarrow \sum_{k \in E}\left|x_{k}\right|=0, \forall E \in \mathcal{N} \Longleftrightarrow\left|x_{k}\right|=0 \quad \forall k \Longleftrightarrow x=0 . \\
& \text { 2) }\|\lambda x\|_{S}=\sup _{E \in X^{\prime}} \sum_{k \in E}\left|\lambda x_{k}\right|=|\lambda| \sup _{E \in \mathcal{N}} \sum_{k \in E}\left|x_{k}\right|=|\lambda|\|x\|_{S}, \forall \lambda \in \mathbb{R} .
\end{aligned}
$$


3) $\|x+y\|_{S}=\sup _{E \in \mathcal{N}} \sum_{k \in E}\left|x_{k}+y_{k}\right| \leq \sup _{E \in \mathcal{N}} \sum_{k \in E}\left(\left|x_{k}\right|+\left|y_{k}\right|\right)=\sup _{E \in \mathcal{N}}\left(\sum_{k \in E}\left|x_{k}\right|+\sum_{k \in E}\left|y_{k}\right|\right) \leq$
$\leq \sup _{E \in \mathcal{N}} \sum_{k \in E}\left|x_{k}\right|+\sup _{E \in \mathcal{X}} \sum_{k \in E}\left|y_{k}\right|=\|x\|_{S}+\|y\|_{S}$.

Proposição 1.3 Seja S o espaço de Schreier.

1) $\forall x=\sum_{k=1}^{\infty} x_{k} e_{k}, x \in c_{00}$ têm-se que $\|x\|_{S} \leq\|x\|_{l_{1}}$.

2) $\left\|e_{n}\right\|_{S}=1, \forall n$.

3) $\left(e_{n}\right)_{n}$ é uma base 1-incondicional para $S$.

4) $\forall x \in c_{00}$ tal que $\operatorname{supp}(x) \in \mathcal{N}$ temos que $\|x\|_{S}=\|x\|_{l_{1}}$.

\section{Demonstração:}

1) $x$ tem suporte finito, então $\|x\|_{S}=\sup _{E \in \mathcal{X}^{\prime}} \sum_{k \in E}\left|x_{k}\right| \leq \sum_{k \in \mathbb{N}}\left|x_{k}\right|=\|x\|_{l_{1}}$.

2) Já sabemos pelo ítem 1 que $\left\|e_{n}\right\|_{S} \leq\left\|e_{n}\right\|_{l_{1}}=1$.

Como $\mathrm{E}=\{n\}$ é admissivel, temos $\left\|e_{n}\right\|_{S}=\sup _{E^{\prime} \in \mathcal{N}} \sum_{k \in E^{\prime}}\left|e_{n}(k)\right| \geq \sum_{k \in E}\left|e_{n}(k)\right|=1$, onde $e_{n}(k)$ representa a coordenada $k$ de $e_{n}$. Portanto $\left\|e_{n}\right\|_{S}=1, \forall n$.

3) Vamos fazer esta demonstração em três partes.

I) $\left(e_{n}\right)_{n}$ é sequência básica.

Pelo Teorema 0.3 é suficiente mostrarmos que

$$
\left\|\sum_{i=1}^{m} a_{i} e_{i}\right\|_{S} \leq\left\|\sum_{i=1}^{n} a_{i} e_{i}\right\|_{S}
$$

para qualquer sequência de escalares $\left(a_{n}\right)_{n}$ e inteiros $m<n$.

Sejam $y=\sum_{i=1}^{m} a_{i} e_{i}, z=\sum_{i=1}^{n} a_{i} e_{i}$ e E um conjunto admissível qualquer. Consideremos $I=\{1,2, \ldots, m\}$ e $J=\{1,2, \ldots, m, m+1, \ldots, n\}$ se $E \cap I \neq \emptyset$, então $\sum_{k \in I \cap E}\left|a_{k}\right| \leq \sum_{k \in J \cap E}\left|a_{k}\right| \leq$ $\|z\|_{S}$. Como a desigualdade vale para qualquer conjunto admissível temos $\|y\|_{S} \leq\|z\|_{S}$, ou seja $\left\|\sum_{i=1}^{m} a_{i} e_{i}\right\|_{S} \leq\left\|\sum_{i=1}^{n} a_{i} e_{i}\right\|_{S}, \forall\left(a_{n}\right)_{n}$ e inteiros $m<n$.

II) $\left(e_{n}\right)_{n}$ gera $\mathrm{S}$.

Como $e_{n} \in S, \forall n \in \mathbb{N}$, claramente $\left[\left(e_{n}\right)_{n}\right] \subset S$. 
$S \subset\left[\left(e_{n}\right)_{n}\right]$, pois dado $y \in S$ existe uma sequência de elementos $y_{n}$ de $c_{00}$ tal que $y_{n} \stackrel{n \rightarrow+\infty}{\rightarrow} y$. Como os $y_{n} \in c_{00}$. eles podem ser escritos como combinação linear de $\left(e_{n}\right)_{n}$ que é base de $c_{00}$. Os $y_{n^{\prime} s}$ formam uma sequência de Cauchy em $\left[\left(e_{n}\right)_{n}\right]$ e portanto $\left(y_{n}\right)_{n}$ é convergente em $\left[\left(e_{n}\right)_{n}\right]$. Como o limite é único temos que $y \in\left[\left(e_{n}\right)_{n}\right]$, logo $S \subset\left[\left(e_{n}\right)_{n}\right]$. Consequentemente $S=\left[\left(e_{n}\right)_{n}\right]$.

III) $\left(e_{n}\right)_{n}$ é 1-incondicional.

Pelo Teorema 0.5 , basta verificarmos que

$$
\left\|\sum_{j \in A} a_{j} e_{j}\right\|_{S} \leq\left\|\sum_{j \in B} a_{j} e_{j}\right\|_{S}
$$

onde $A$ e $B$ são subconjuntos finitos dos naturais com $A \subset B$.

Seja $E$ um conjunto admissível qualquer. Se $A \cap E \neq \emptyset$. então $\sum_{j \in A \cap E}\left|a_{j}\right| \leq \sum_{j \in B \cap E}\left|a_{j}\right| \leq$ $\left\|\sum_{j \in B} a_{j} e_{j}\right\|_{s}$. Como a desigualdade vale para qualquer conjunto admissível temos $\left\|\sum_{j \in A} a_{j} e_{j}\right\|_{S} \leq\left\|\sum_{j \in B} a_{j} e_{j}\right\|_{S}$, onde $A$ e $B$ são subconjuntos finitos dos naturais com $A \subset B$.

Por I), II) e III) temos que $\left(e_{n}\right)_{n}$ é base 1-incondicional para $\mathrm{S}$.

$$
\begin{aligned}
& \text { 4) Sejam } x=\sum_{i=1}^{\infty} a_{i} e_{i} \text { e } L=\operatorname{supp}(x) \in \mathcal{N} \text {, temos que } \sum_{i \in L}\left|a_{i}\right|=\|x\|_{l_{1}} \text { mas, } \sup _{E \in \mathcal{N}_{i \in E}}\left|a_{i}\right| \geq \\
& \sum_{i \in L}\left|a_{i}\right|=\|x\|_{l_{1}} \text {. Logo }\|x\|_{S} \geq\|x\|_{l_{1}} \text { e como por 1) }\|x\|_{S} \leq\|x\|_{l_{1}} \text { temos o resultado. }
\end{aligned}
$$

Teorema $1.4 l_{1}(\mathbb{N}) \leftrightarrow S$.

\section{Demonstração:}

Se $l_{1}(\mathbb{N}) \hookrightarrow S$, tomando $\delta=\frac{1}{10}$, teriamos pelo Teorema 0.14 que existe uma sequência normalizada de blocos $\left(u_{n-1}\right)_{n}$ de $\left(e_{n}\right)_{n}$ para a qual

$$
\frac{9}{10} \sum_{i=0}^{\infty}\left|a_{i}\right| \leq\left\|\sum_{i=0}^{\infty} a_{i} u_{i}\right\|_{S}
$$

para toda sequência finita de escalares $\left(a_{i-1}\right)_{i}$.

Logo $\frac{18}{10} \leq\left\|u_{0}+\frac{1}{n}\left(u_{1}+\ldots+u_{n}\right)\right\|_{S}, \forall n \geq 1$. E então basta usarmos a Proposição abaixo para obtermos um absurdo. 
Proposição 1.5 Para cada sequéncia normalizada de blocos consecutivos $\left(u_{n-1}\right)_{n}$ de $\left(e_{n}\right)_{n}$ temos

$$
\varlimsup_{n}\left\|u_{0}+\frac{1}{n}\left(u_{1}+\ldots+u_{n}\right)\right\|_{S} \leq 1
$$

\section{Demonstração:}

Para cada $n \geq 1$ consideremos a quantidade $\left\|u_{0}+\frac{1}{n}\left(u_{1}+\ldots+u_{n}\right)\right\|_{S}$, e seja $E \in \mathcal{N}$ tal que $\left\|u_{0}+\frac{1}{n}\left(u_{1}+\ldots+u_{n}\right)\right\|_{S}=\sum_{k \in E}\left|u_{0}(k)+\frac{1}{n}\left(u_{1}(k)+\ldots+u_{n}(k)\right)\right|$, onde $u_{n}(k)$ representa a coordenada $k$ de $u_{n}$.

Se $E$ não encontra $\operatorname{supp}\left(u_{0}\right)$. isto é, $E$ é disjunto do $\operatorname{supp}\left(u_{0}\right)$ então $u_{0}(k)=0, \forall k \in E$ e $\frac{1}{n}\left(u_{1}(k)+\ldots+u_{n}(k)\right)$ é no máximo 1 .

Se $E$ encontra $\operatorname{supp}\left(u_{0}\right)$ seja $|E|$ a cardinalidade de $E .|E|$ independe de $n$, pois só devemos ter $|E| \leq n_{1}$ onde $n_{1}$ é a primeira coordenada do $\operatorname{supp}\left(u_{0}\right)$ que $E$ encontra, e assim

$$
\lim _{n} \sum_{k \in E}\left|\frac{1}{n}\left(u_{1}(k)+\ldots+u_{n}(k)\right)\right|=0
$$

pois sejam $u_{1}, u_{2}, \ldots, u_{p}$ os vetores que encontram $E, p \leq n$ então

$$
\lim _{n \rightarrow+\infty} \frac{u_{1}(k)+\ldots+u_{p}(k)}{n} \leq \lim _{n \rightarrow+\infty} \frac{p}{n}=0
$$

E assim temos o resultado.

Teorema $1.6 c_{0}(\mathbb{N}) \hookrightarrow S$.

\section{Demonstração:}

Vamos produzir uma sequência $\left(u_{n}\right)_{n}$ em $\mathrm{S}$ equivalente a base canônica de $c_{0}(\mathbb{N})$, definindo:

$u_{1}=e_{1} ; u_{2}=\frac{e_{2}+e_{3}}{2} ; \ldots$

$$
u_{n}=2^{n-1}\left(\sum_{k=2^{n-1}}^{2^{n}-1} e_{k}\right), n \geq 3
$$

Podemos observar que

1 ) os suportes de $\left(u_{n}\right)_{n}$ são conjuntos admissiveis. 
2) $\left(u_{n}\right)_{n}$ é uma sequência de blocos de $\left(e_{n}\right)_{n}$.

3) $\left\|u_{n}\right\|_{S}=1 \forall n$, pois pelo ítem 1 e pela Proposição 1.3 ítem $4\left\|u_{n}\right\|_{S}=\left\|u_{n}\right\|_{l_{1}}=1$.

Seja $\left(a_{i}\right)_{i}$ uma sequência finita de escalares, logo

$$
\left|a_{n}\right| \leq\left\|\sum_{i=1}^{k} a_{i} u_{i}\right\|_{S}, \forall n, 1 \leq n \leq k
$$

pois se $N_{n}$ é o suporte de $u_{n}$. então $N_{n}$ é admissivel e portanto

$$
\left\|\sum_{i=1}^{k} a_{i} u_{i}\right\|_{S}=\sup _{E \in \mathcal{N}} \sum_{l \in E}\left|b_{l}\right| \geq \sum_{l \in N_{n}}\left|b_{l}\right|=\left|a_{n}\right|
$$

onde $b_{l}$ é da forma $\frac{a_{j}}{2^{j-1}}, \forall j, 1 \leq j \leq k$.

Logo

$$
\left\|\sum_{i=1}^{k} a_{i} u_{i}\right\|_{S} \geq \max _{1 \leq n \leq k}\left|a_{n}\right|
$$

Por outro lado, pela Proposição 1.3

$$
\left\|\sum_{i=1}^{k} a_{i} u_{i}\right\|_{S} \leq\left\|\sum_{i=1}^{k}\left|a_{i}\right| u_{i}\right\|_{S} \leq\left\|\sum_{i=1}^{k}\left(\max _{1 \leq l \leq k}\left|a_{l}\right|\right) u_{i}\right\|_{S}=\max _{1 \leq l \leq k}\left|a_{l}\right|\left\|\sum_{i=1}^{k} u_{i}\right\|_{S}
$$

e então é suficiente mostrarmos que $\left\|\sum_{i=1}^{k} u_{i}\right\|_{S}=1$.

Para isto seja $\bar{u}_{k}=\sum_{i=1}^{k} u_{i}$ e considere $E \in \mathcal{N}$ tal que $\left\|\bar{u}_{k}\right\|_{S}=\sum_{j \in E}\left|\bar{u}_{k}(j)\right|$.

Sem perda de generalidade, $E$ pode ser tomado como um segmento de números naturais $E=\{l, l+1, l+2, \ldots \ldots, 2 l-1\}$.

Desde que $\bar{u}_{k}=\sum_{i=1}^{2^{k}-1} a_{i} e_{i}$, onde $a_{i}$ toma valores $1, \frac{1}{2}, \frac{1}{4}$, etc, existe $k_{0}$ tal que $2^{k_{0}} \leq l<$ $2^{k_{0}+1}$ e $\left\|\bar{u}_{k}\right\|_{S}=\sum_{j \in E}\left|\bar{u}_{k}(j)\right|=\frac{2^{k_{0}+1}-l}{2^{k_{0}}}+\frac{2 l-2^{k_{0}+1}}{2^{k_{0}+1}}=2^{-k_{0}}\left(2^{k_{0}+1}-l\right)+2^{-k_{0}-1}\left(2 l-2^{k_{0}+1}\right)=$ 1.

Portanto $\left\|\bar{u}_{k}\right\|_{S}=1$ e $\left\|\sum_{i=1}^{k} a_{i} u_{i}\right\|_{S} \leq \max _{1 \leq l \leq k}\left|a_{l}\right|$ como queriamos.

Logo $\left(u_{n}\right)_{n}$ é equivalente a base canônica de $c_{0}(\mathbb{N})$ e portanto $c_{0}(\mathbb{N}) \hookrightarrow S$. 


\subsection{O espaço de Baernstein}

Para todo $x \in c_{00}$, definimos

$$
\|x\|_{B}=\sup \left\{\left(\sum_{k=1}^{n}\left\|E_{k} x\right\|_{l_{1}}^{2}\right)^{1 / 2}: E_{k} \in \mathcal{N}, E_{1}<E_{2}<\ldots<E_{n}, n=1,2, \ldots\right\}
$$

isto é, o supremo é tomado sobre todos subconjuntos finitos consecutivos $E_{k} \in \mathbb{N}$ tais que $\left|E_{k}\right| \leq \min \left(E_{k}\right)$.

O espaço de Baernstein, denotado por B, é definido como sendo o completado de $c_{00}$ com a norma $\|\cdot\|_{B}$.

Notemos que $\|\cdot\|_{B}$ é norma em $c_{00}$, de fato sejam $x$ e $y$ elementos de $c_{00}$.

1) claramente $\|x\|_{B} \geq 0$.

$\|x\|_{B}=0 \Longleftrightarrow \sup \left\{\left(\sum_{k=1}^{n}\left\|E_{k} x\right\|_{l_{1}}^{2}\right)^{1 / 2}, E_{1}<\ldots<E_{n}\right\}=0 \Longleftrightarrow\left(\sum_{k=1}^{n}\left\|E_{k} x\right\|_{l_{1}}^{2}\right)^{1 / 2}=0 \quad \forall E_{1}<$ $\ldots<E_{n} \Longleftrightarrow \sum_{k=1}^{n}\left\|E_{k} x\right\|_{l_{1}}^{2}=0 \Longleftrightarrow\left\|E_{k} x\right\|_{l_{1}}=0, \quad 1 \leq k \leq n \Longleftrightarrow E_{k} x=0 \quad 1 \leq k \leq n \Longleftrightarrow$ $x=0$.

$$
\begin{aligned}
& \text { 2) }\|\lambda x\|_{B}=\sup \left\{\left(\sum_{k=1}^{n}\left\|E_{k} \lambda x\right\|_{l_{1}}^{2}\right)^{1 / 2}, E_{1}<\ldots<E_{n}\right\}=\sup \left\{|\lambda|\left(\sum_{k=1}^{n}\left\|E_{k} x\right\|_{l_{1}}^{2}\right)^{1 / 2}, E_{1}<\right. \\
& \left.\ldots<E_{n}\right\}=|\lambda| \sup \left\{\left(\sum_{k=1}^{n}\left\|E_{k} x\right\|_{l_{1}}^{2}\right)^{1 / 2}, E_{1}<\ldots<E_{n}\right\}=|\lambda|\|x\|_{B}, \forall \lambda \in \mathbb{R} . \\
& \text { 3) }\left(\sum_{k=1}^{n}\left\|E_{k}(x+y)\right\|_{l_{1}}^{2}\right)^{1 / 2}=\left(\sum_{k=1}^{n}\left\|E_{k} x+E_{k} y\right\|_{l_{1}}^{2}\right)^{1 / 2} \leq\left(\sum_{k=1}^{n}\left(\left\|E_{k} x\right\|_{l_{1}}+\left\|E_{k} y\right\|_{l_{1}}\right)^{2}\right)^{1 / 2} \leq \\
& \left(\sum_{k=1}^{n}\left\|E_{k} x\right\|_{l_{1}}^{2}\right)^{1 / 2}+\left(\sum_{k=1}^{n}\left\|E_{k} y\right\|_{l_{1}}^{2}\right)^{1 / 2} \leq \sup \left\{\left(\sum_{k=1}^{n}\left\|E_{k} x\right\|_{l_{1}}^{2}\right)^{1 / 2}, E_{1}<\ldots<E_{n}\right\}+ \\
& \sup \left\{\left(\sum_{k=1}^{n}\left\|E_{k} y\right\|_{l_{1}}^{2}\right)^{1 / 2}, E_{1}<\ldots<E_{n}\right\}
\end{aligned}
$$

Como a desigualdade vale para qualquer elemento da forma $\left(\sum_{k=1}^{n}\left\|E_{k}(x+y)\right\|_{l_{1}}^{2}\right)^{1 / 2}$ temos que $\sup \left\{\left(\sum_{k=1}^{n}\left\|E_{k}(x+y)\right\|_{l_{1}}^{2}\right)^{1 / 2}, E_{1}<\ldots<E_{n}\right\} \leq \sup \left\{\left(\sum_{k=1}^{n}\left\|E_{k} x\right\|_{l_{1}}^{2}\right)^{1 / 2}, E_{1}<\ldots<E_{n}\right\}+$ $\sup \left\{\left(\sum_{k=1}^{n}\left\|E_{k} y\right\|_{l_{1}}^{2}\right)^{1 / 2}, E_{1}<\ldots<E_{n}\right\}$, ou seja, $\|x+y\|_{B} \leq\|x\|_{B}+\|y\|_{B}$. 
Proposição 1.7 Seja B o espaço de Baernstein.

a) $\left\|e_{n}\right\|_{B}=1, \forall n \in \mathbb{N}$.

b) $\left(e_{n}\right)_{n}$ é uma base 1-incondicional para $B$.

\section{Demonstração:}

a) $\left\|e_{n}\right\|_{B}=\sup \left\{\left(\sum_{k=1}^{N}\left\|E_{k} e_{n}\right\|_{l_{1}}^{2}\right)^{1 / 2}, E_{1}<\ldots<E_{N}\right\}$ e $e_{n}=\{0, \ldots, 0, \underbrace{1}_{n}, 0, \ldots\}$. Então seja qual for a sequência de conjuntos admíssiveis consecutivos considerada, nós teremos que no máximo um deles vai conter a coordenada não nula de $e_{n}$ ou talvez nenhum deles intercepte $e_{n}$ na coordenada não nula. Sendo assim temos duas possibilidades $\left\|E_{k} e_{n}\right\|_{l_{1}}=$ 1 ou $\left\|E_{k} e_{n}\right\|_{l_{1}}=0$. Portanto $\left\|e_{n}\right\|_{B}=1$ para qualquer $n \in \mathbb{N}$.

b) Vamos fazer a demonstração em três partes.

1) $\left(e_{n}\right)_{n}$ é sequência básica.

Pelo Teorema 0.3 é suficiente mostrarmos que

$$
\left\|\sum_{i=1}^{m} a_{i} e_{i}\right\|_{B} \leq\left\|\sum_{i=1}^{n} a_{i} e_{i}\right\|_{B}
$$

para qualquer sequência de escalares $\left(a_{n}\right)_{n}$ e inteiros $m<n$.

Consideremos $y=\sum_{i=1}^{m} a_{i} e_{i}, z=\sum_{i=1}^{n} a_{i} e_{i}$ e $\left\{E_{k}\right\}_{k=1}^{l}$ uma sequência qualquer de conjuntos admissíveis consecutivos. Então temos

$$
\left(\sum_{k=1}^{l}\left\|E_{k} y\right\|_{l_{1}}^{2}\right)^{1 / 2} \leq\left(\sum_{k=1}^{l}\left\|E_{k} z\right\|_{l_{1}}^{2}\right)^{1 / 2} \leq \sup \left\{\left(\sum_{k=1}^{l}\left\|E_{k} z\right\|_{l_{1}}^{2}\right)^{1 / 2}, E_{1}<E_{2}<\ldots\right\}=\|z\|_{B} .
$$

Como essa desigualdade vale para qualquer sequência de conjuntos admissíveis consecutivos temos que $\|y\|_{B} \leq\|z\|_{B}$.

2) $\left(e_{n}\right)_{n}$ gera $\mathrm{B}$.

Demonstração análoga a feita para o espaço de Schreier.

3) $\left(e_{n}\right)_{n}$ é 1-incondicional para B.

Pelo Teorema 0.5 , basta verificarmos que

$$
\left\|\sum_{i \in A} a_{i} e_{i}\right\|_{B} \leq\left\|\sum_{i \in B} a_{i} e_{i}\right\|_{B}
$$


onde $A$ e $B$ são subconjuntos finitos dos naturais com $A \subset B$.

Consideremos $y=\sum_{i \in A} a_{i} e_{i}, z=\sum_{i \in B} a_{i} e_{i}$ e $\left\{E_{k}\right\}_{k=1}^{l}$ uma sequência qualquer de conjuntos admissíveis consecutivos. Então temos

$$
\left(\sum_{k=1}^{l}\left\|E_{k} y\right\|_{l_{1}}^{2}\right)^{1 / 2} \leq\left(\sum_{k=1}^{l}\left\|E_{k} z\right\|_{l_{1}}^{2}\right)^{1 / 2} \leq \sup \left\{\left(\sum_{k=1}^{l}\left\|E_{k} z\right\|_{l_{1}}^{2}\right)^{1 / 2}, E_{1}<E_{2}<\ldots\right\}=\|z\|_{B} .
$$

Como essa desigualdade vale para qualquer sequência de conjuntos admissíveis consecutivos temos que $\|y\|_{B} \leq\|z\|_{B}$.

Por 1$), 2$ ) e 3 ) temos que $\left(e_{n}\right)_{n}$ é base 1-incondicional para B.

Para demonstrarmos o próximo Teorema precisamos da Observação abaixo e da Proposição 1.9 .

Observação 1.8 Seja $\left(a_{n}\right)_{n}$ uma sequência de números reais tais que $\left(\sum_{k=1}^{\infty}\left\|E_{k}\left(\sum_{n=1}^{\infty} a_{n} e_{n}\right)\right\|_{l_{1}}^{2}\right)^{1 / 2} \leq 1$ para toda sequência $\left\{E_{k}\right\}_{k=1}^{\infty}$ de subconjuntos admissíveis consecutivos dos naturais. Então $x=\sum_{n=1}^{\infty} a_{n} e_{n}$ é convergente em B.

De fato, basta demonstrarmos que $S_{n}=a_{1} e_{1}+\ldots+a_{n} \epsilon_{n}$ é de Cauchy em B.

Suponhamos que $S_{n}$ não é de Cauchy, então existe $\epsilon>0$ tal que para qualquer $r \in \mathbb{N}$. existe $p<q, p, q \geq r$ com $\left\|S_{p}-S_{q}\right\|_{B}=\left\|a_{p+1} e_{p+1}+\ldots+a_{q} e_{q}\right\|_{B} \geq \epsilon$, ou seja, existe $E_{1}<\ldots<E_{n}, E_{i} \in \mathcal{N}$ satisfazendo $\sum_{i=1}^{n}\left\|E_{i}\left(a_{p+1} e_{p+1}+\ldots+a_{q} e_{q}\right)\right\|_{l_{1}}^{2} \geq \epsilon^{2}$.

Seja $N \in \mathbb{N} \operatorname{com} \epsilon^{2} N>1$. Tomemos $m_{1}=1$, como $S_{n}$ não é Cauchy existe $p_{1}, q_{1} \geq m_{1}$ tal que $\sum_{i=1}^{n_{1}}\left\|E_{i}\left(a_{p_{1}+1} e_{p_{1}+1}+\ldots+a_{q_{1}} e_{q_{1}}\right)\right\|_{l_{1}}^{2} \geq \epsilon^{2}$. Seja $m_{2}=\max \left(E_{n_{1}}\right)+1$ logo existe $p_{2}, q_{2} \geq m_{2}$ tal que $\sum_{i=n_{1}+1}^{n_{2}}\left\|E_{i}\left(a_{p_{2}+1} e_{p_{2}+1}+\ldots+a_{q_{2}} e_{q_{2}}\right)\right\|_{l_{1}}^{2} \geq \epsilon^{2}$.

Analogamente conseguimos $E_{1}<E_{2}<\ldots<E_{n_{N}}$ tais que $\sum_{i=1}^{n_{N}} \| E_{i}\left(a_{p_{1}+1} e_{p_{1}+1}+\ldots+\right.$ $\left.a_{q_{N}} e_{q_{N}}\right)\left\|_{l_{1}}^{2}=\sum_{i=1}^{n_{1}}\right\| E_{i}\left(a_{p_{1}+1} \epsilon_{p_{1}+1}+\ldots+a_{q_{N}} e_{q_{N}}\right)\left\|_{l_{1}}^{2}+\ldots+\sum_{i=n_{N-1}+1}^{n_{N}}\right\| E_{i}\left(a_{p_{1}+1} e_{p_{1}+1}+\ldots+\right.$ 
$\left.a_{q_{N}} e_{q_{N}}\right) \|_{l_{1}}^{2} \geq N \epsilon^{2}>1$.

Absurdo, pois por hipótese $\left(\sum_{k=1}^{\infty}\left\|E_{k}\left(\sum_{n=1}^{\infty} a_{n} e_{n}\right)\right\|_{l_{1}}^{2}\right)^{1 / 2} \leq 1$ para toda sequência $\left\{E_{k}\right\}_{k=1}^{\infty}$ de subconjuntos admissiveis consecutivos dos naturais.

Proposição 1.9 Para toda sequência de blocos normalizada consecutiva $\left(u_{n-1}\right)_{n}$ de $\left(e_{n}\right)_{n}$, temos

$$
\varlimsup_{n}\left\|u_{0}+\frac{1}{n}\left(u_{1}+u_{2}+\ldots+u_{n}\right)\right\|_{B} \leq \sqrt{2}
$$

\section{Demonstração:}

Fixemos $n \geq 1$ e consideremos uma sequência de subconjuntos admissíveis consecutivos dos naturais, digamos $E_{1}<E_{2}<\ldots$.

Se $\operatorname{supp}\left(u_{0}\right)<E_{1}$. entào

$\left(\sum_{k=1}^{\infty}\left\|E_{k}\left(u_{0}+\frac{1}{n}\left(u_{1}+\ldots+u_{n}\right)\right)\right\|_{l_{1}}^{2}\right)^{1 / 2}=\left(\sum_{k=1}^{\infty}\left\|E_{k}\left(\frac{1}{n}\left(u_{1}+\ldots+u_{n}\right)\right)\right\|_{l_{1}}^{2}\right)^{1 / 2}=\frac{1}{n}\left(\sum_{k=1}^{\infty} \| E_{k}\left(u_{1}+\right.\right.$
$\left.\left.\ldots+u_{n}\right) \|_{l_{1}}^{2}\right)^{1 / 2} \leq \frac{1}{n}\left\|u_{1}+\ldots+u_{n}\right\|_{B} \leq \frac{1}{n}\left(\left\|u_{1}\right\|_{B}+\ldots+\left\|u_{n}\right\|_{B}\right) \leq \frac{n}{n}=1$.

Caso contrário, sejam $k_{1}$ o maior índice $k$ para o qual $\min E_{k} \leq \max \operatorname{supp}\left(u_{0}\right)$ e $f\left(u_{0}\right)=\max \operatorname{supp}\left(u_{0}\right)$. Entào $\left|E_{k_{1}}\right| \leq \min E_{k_{1}} \leq f\left(u_{0}\right)$ e portanto,

$\sum_{k=1}^{k_{1}}\left\|E_{k}\left(u_{0}+\frac{1}{n}\left(u_{1}+\ldots+u_{n}\right)\right)\right\|_{i_{1}}^{2}=\sum_{k=1}^{k_{1}-1}\left\|E_{k}\left(u_{0}+\frac{1}{n}\left(u_{1}+\ldots+u_{n}\right)\right)\right\|_{l_{1}}^{2}+\| E_{k_{1}}\left(u_{0}+\frac{1}{n}\left(u_{1}+\ldots+\right.\right.$ $\left.\left.u_{n}\right)\right)\left\|_{l_{1}}^{2}=\sum_{k=1}^{k_{1}-1}\right\| E_{k}\left(u_{0}\right)\left\|_{l_{1}}^{2}+\right\| E_{k_{1}}\left(u_{0}+\frac{1}{n}\left(u_{1}+\ldots+u_{n}\right)\right) \|_{l_{1}}^{2} \leq\left(\sum_{k=1}^{k_{1}-1}\left\|E_{k}\left(u_{0}\right)\right\|_{l_{1}}+\| E_{k_{1}}\left(u_{0}+\right.\right.$ $\left.\left.\frac{1}{n}\left(u_{1}+\ldots+u_{n}\right)\right) \|_{l_{1}}\right)^{2} \leq\left(\sum_{k=1}^{k_{1}-1}\left\|E_{k}\left(u_{0}\right)\right\|_{l_{1}}+\left\|E_{k_{1}}\left(u_{0}\right)\right\|_{l_{1}}+\left\|E_{k_{1}}\left(\frac{1}{n}\left(u_{1}+\ldots+u_{n}\right)\right)\right\|_{l_{1}}\right)^{2}=$ $\left(\sum_{k=1}^{k_{1}}\left\|E_{k}\left(u_{0}\right)\right\|_{l_{1}}+\left\|E_{k_{1}}\left(\frac{1}{n}\left(u_{1}+\ldots+u_{n}\right)\right)\right\|_{l_{1}}\right)^{2} \leq\left(\left\|u_{0}\right\|_{B}+\left\|E_{k_{1}}\left(\frac{1}{n}\left(u_{1}+\ldots+u_{n}\right)\right)\right\|_{B}\right)^{2} \leq$ $\left(1+\frac{1}{n} \sum_{i=1}^{n}\left\|E_{k_{1}}\left(u_{i}\right)\right\|_{B}\right)^{2} \leq\left(1+\frac{1}{n} f\left(u_{0}\right)\right)^{2}$.

Observação: $\left\|E_{k_{1}}\left(u_{1}\right)\right\|_{B}+\ldots+\left\|E_{k_{1}}\left(u_{n}\right)\right\|_{B} \leq f\left(u_{0}\right)$. De fato, seja $A=\left\{i: E_{k_{1}} \cap \operatorname{supp}\left(u_{i}\right) \neq\right.$ $\emptyset$ ฤ. Temos $|A| \leq\left|E_{k_{1}}\right| \leq f\left(u_{0}\right)$ e $\left\|E_{k_{1}}\left(u_{j}\right)\right\|_{B} \leq\left\|u_{j}\right\|_{B} \leq 1$.

Logo $\left\|E_{k_{1}}\left(u_{1}\right)\right\|_{B}+\ldots+\left\|E_{k_{1}}\left(u_{n}\right)\right\|_{B} \leq|A|\left\|u_{j}\right\|_{B} \leq f\left(u_{0}\right)$. 


$$
\begin{aligned}
& \text { Portanto }\left(\sum_{k=1}^{\infty}\left\|E_{k}\left(u_{0}+\frac{1}{n}\left(u_{1}+\ldots+u_{n}\right)\right)\right\|_{l_{1}}^{2}\right)^{1 / 2}=\left(\sum_{k=1}^{k_{1}}\left\|E_{k}\left(u_{0}+\frac{1}{n}\left(u_{1}+\ldots+u_{n}\right)\right)\right\|_{l_{1}}^{2}+\right. \\
& \sum_{k=k_{1}+1}^{\infty} \| E_{k}\left(\frac{1}{n}\left(u_{1}+\ldots+u_{n}\right) \|_{l_{1}}^{2}\right)^{1 / 2} \leq\left(\left(1+\frac{1}{n} f\left(u_{0}\right)\right)^{2}+1\right)^{1 / 2} .
\end{aligned}
$$$$
\text { Assim }\left\|u_{0}+\frac{1}{n}\left(u_{1}+\ldots+u_{n}\right)\right\|_{B} \leq\left(\left(1+\frac{1}{n} f\left(u_{0}\right)\right)^{2}+1\right)^{1 / 2} .
$$

Consequentemente $\varlimsup_{n}\left\|u_{0}+\frac{1}{n}\left(u_{1}+\ldots+u_{n}\right)\right\|_{B} \leq \sqrt{2}$.

Teorema 1.10 Para o espaço de Baernstein B temos

a) $l_{1}(\mathbb{N}) \leftrightarrow B$.

b) $c_{0}(\mathbb{N}) \leftrightarrow B$.

c) B é reflexivo.

\section{Demonstração:}

a) Análoga a demonstração do Teorema 1.4 e usando a Proposição acima.

b) Pelo Teorema 0.12 , basta mostrarmos que $\left(\epsilon_{n}\right)_{n}$ é limitadamente completa. Para isso precisamos mostrar que para qualquer sequência de escalares $x=\left(x_{i}\right)_{i}$ se o $\sup _{n}\left\|\sum_{i=1}^{n} x_{i} e_{i}\right\|_{B} \leq 1$, entào $x$ é convergente em B.

Para este fim, seja $\left\{E_{k}\right\}_{k=1}^{\infty}$ uma sequência de subconjuntos admissiveis consecutivos dos naturais. Para cada $\kappa^{\circ} \in \mathbb{N}$,

$$
\begin{aligned}
& \left(\sum_{k=1}^{K}\left\|E_{k} x\right\|_{l_{1}}^{2}\right)^{1 / 2} \leq\|\underbrace{\left(\cup_{k=1}^{K} E_{k}\right) x}_{y}\|_{B}=\sup \left\{\left(\sum_{i=1}^{n}\left\|E_{i} y\right\|_{l_{1}}^{2}\right)^{1 / 2}, E_{1}<E_{2}<\ldots\right\} \leq \\
& \sup _{n}\left\|\sum_{i=1}^{n} x_{i} e_{i}\right\|_{B} \leq 1 . A \operatorname{ssim}\left(\sum_{k=1}^{\infty}\left\|E_{k} x\right\|_{l_{1}}^{2}\right)^{1 / 2} \leq 1 .
\end{aligned}
$$

Como $\left\{E_{k}\right\}_{k=1}^{\infty}$ é uma sequência qualquer temos $\sup \left\{\left(\sum_{k=1}^{\infty}\left\|E_{k} x\right\|_{l_{1}}^{2}\right)^{1 / 2}, E_{1}<E_{2}<\right.$ $<\ldots\} \leq 1$. Portanto pela Observação 1.8 temos que $x$ é convergente em $\mathrm{B}$.

c) Segue imediatamente dos ítens a e b e do Teorema 0.13 , pois como vimos B tem uma base incondicional. 


\subsection{O espaço de Tsirelson}

Vamos definir indutivamente uma sequência de normas $\left(\|\cdot\|_{m}\right)_{m}$ em $c_{00}$ como segue.

Seja $x=\sum_{n=1}^{\infty} a_{n} e_{n} \in c_{00}$, definimos $\|x\|_{0}=\max _{n}\left|a_{n}\right| \mathrm{e}$ $\|x\|_{m+1}=\max \left\{\|x\|_{m}, \frac{1}{2} \max \left[\sum_{j=1}^{k}\left\|E_{j} x\right\|_{m}\right]\right\}$, para $m \geq 0$

onde o segundo máximo é tomado sobre todas as escolhas de subconjuntos finitos $\left\{E_{j}\right\}_{j=1}^{k}$ de $\mathbb{N}, k \in \mathbb{N}$ tal que $k \leq E_{1}<E_{2}<\ldots<E_{k}$.

Notemos que $\|x\|_{m}$ é norma em $c_{00}$. De fato, sejam $x$ e $y$ elementos de $c_{00}$.

Já sabemos que $\|x\|_{0}=\max _{n}\left|a_{n}\right|$ é norma em $c_{00}$. Vamos assumir que $\|x\|_{m}$ é norma em $c_{00}$ e provarmos que $\|x\|_{m+1}^{n}$ é norma em $c_{00}$.

1) claramente $\|x\|_{m+1} \geq 0$.

$\|x\|_{m+1}=0 \Longleftrightarrow \max \left\{\|x\|_{m}, \frac{1}{2} \max \left[\sum_{j=1}^{k}\left\|E_{j} x\right\|_{m}\right]\right\}=0 \Longleftrightarrow x=0$.

2) $\|\lambda x\|_{m+1}=\max \left\{\|\lambda x\|_{m}, \frac{1}{2} \max \left[\sum_{j=1}^{k}\left\|E_{j} \lambda x\right\|_{m}\right]\right\}=\max \left\{|\lambda|\|x\|_{m}\right.$,

$\left.|\lambda| \frac{1}{2} \max \left[\sum_{j=1}^{k}\left\|E_{j} x\right\|_{m}\right]\right\}=|\lambda| \max \left\{\|x\|_{m}, \frac{1}{2} \max \left[\sum_{j=1}^{k}\left\|E_{j} x\right\|_{m}\right]\right\}=|\lambda|\|x\|_{m+1}, \forall \lambda \in \mathbb{R}$.

3) $\|x+y\|_{m+1}=\max \left\{\|x+y\|_{m}, \frac{1}{2} \max \left[\sum_{j=1}^{k}\left\|E_{j}(x+y)\right\|_{m}\right]\right\}$.

caso 1:

$\|x+y\|_{m+1}=\|x+y\|_{m} \leq\|x\|_{m}+\|y\|_{m} \leq\|x\|_{m+1}+\|y\|_{m+1}$.

caso 2:

$\|x+y\|_{m+1}=\frac{1}{2} \max \left[\sum_{j=1}^{k}\left\|E_{j}(x+y)\right\|_{m}\right] \leq \frac{1}{2} \max \left[\sum_{j=1}^{k}\left\|E_{j} x\right\|_{m}+\sum_{j=1}^{k}\left\|E_{j} y\right\|_{m}\right] \leq$

$\frac{1}{2} \max \left[\sum_{j=1}^{k}\left\|E_{j} x\right\|_{m}\right]+\frac{1}{2} \max \left[\sum_{j=1}^{k}\left\|E_{j} y\right\|_{m}\right] \leq\|x\|_{m+1}+\|y\|_{m+1}$.

Portanto $\|x+y\|_{m+1} \leq\|x\|_{m+1}+\|y\|_{m+1}$. 
Vamos destacar agora duas propriedades de $\|x\|_{m}$.

1) $\left(\|\cdot\|_{m}\right)_{m}$ é crescente com $m$.

De fato, pela definição de norma temos $\|x\|_{m+1} \geq\|x\|_{m}, \forall m \geq 0$.

2) $\|x\|_{m} \leq\|x\|_{\iota_{1}}$ para todo $x \in c_{00}$ e para todo $m \geq 0$.

De fato, $\|x\|_{0}=\max _{n}\left|a_{n}\right| \leq \sum_{n=1}^{\infty}\left|a_{n}\right|=\|x\|_{l_{1}}$.

Vamos assumir agora que $\|x\|_{m} \leq\|x\|_{l_{1}}$ e provarmos que $\|x\|_{m+1} \leq\|x\|_{l_{1}}$.

caso 1:

$$
\|x\|_{m+1}=\max \left\{\|x\|_{m}, \frac{1}{2} \max \left[\sum_{j=1}^{k}\left\|E_{j} x\right\|_{m}\right]\right\}
$$

$\|x\|_{m+1}=\|x\|_{m} \leq\|x\|_{l_{1}}$.

caso 2:

$\|x\|_{m+1}=\frac{1}{2} \max \left[\sum_{j=1}^{k}\left\|E_{j} x\right\|_{m}\right] \leq \frac{1}{2} \max \left[\sum_{j=1}^{k}\left\|E_{j} x\right\|_{l_{1}}\right] \leq \frac{1}{2}\|x\|_{l_{1}} \leq\|x\|_{l_{1}}$.

Portanto $\|x\|_{m+1} \leq\|x\|_{l_{1}}$, com isto temos que $\|x\|_{m} \leq\|x\|_{l_{1}}, \forall m \geq 0$.

De 1) e 2) segue que, para cada $x \in c_{00}, \lim _{m \rightarrow+\infty}\|x\|_{m}$ existe e é majorado pela $\|x\|_{l_{1}}$. Nós denotaremos $\lim _{m \rightarrow+\infty}\|x\|_{m}$ por $\|x\|$, e vamos verificar que $\|x\|$ é norma em $c_{00}$. De fato, sejam $x$ e $y$ elementos de $c_{00}$.

1) claramente $\|x\| \geq 0$.

$\|x\|=0 \Longleftrightarrow \lim _{m}\|x\|_{m}=0 \Longleftrightarrow\|x\|_{m}=0, \quad \forall m \Longleftrightarrow x=0$.

2) $\|\lambda x\|=\lim _{m}\|\lambda x\|_{m}=\lim _{m}|\lambda|\|x\|_{m}=|\lambda| \lim _{m}\|x\|_{m}=|\lambda|\|x\|, \forall \lambda \in \mathbb{R}$.

3) $\|x+y\|=\lim _{m}\|x+y\|_{m} \leq \lim _{m}\|x\|_{m}+\lim _{m}\|y\|_{m}=\|x\|+\|y\|$.

$\mathrm{O}$ espaço de Tsirelson, denotado por $\mathrm{T}$, é definido como sendo o completado de $c_{00}$ com a norma acima.

Antes de obtermos as propriedades citadas no início deste capítulo precisamos da seguinte Proposição.

Proposição 1.11 Para o espaço de Tsirelson temos:

1) A sequência $\left(e_{n}\right)_{n}$ forma uma base de Schauder normalizada 1-incondicional para o espaço $T$. 
2) Para qualquer $x \in c_{00}$. e qualquer $m \geq 0$ temos

$$
\begin{aligned}
& \|x\|_{m+1}=\sup \left\{\frac{1}{2} \sum_{j=1}^{k}\left\|E_{j} x\right\|_{m}: k \leq E_{1}<E_{2}<\ldots<E_{k}, k=1,2, \ldots\right\} \\
& \text { ou }\|x\|_{m+1}=\|x\|_{0} .
\end{aligned}
$$

3) Para cada $x=\sum_{n=1}^{\infty} a_{n} e_{n} \in T$, temos $\|x\|=\max \left\{\max _{n}\left|a_{n}\right|, \frac{1}{2} s u p \sum_{j=1}^{k}\left\|E_{j} x\right\|\right\}$, onde o supremo é tomado sobre todas as sequências de subconjuntos finitos $\left\{E_{j}\right\}_{j=1}^{k}$ dos naturais, onde $k \in \mathbb{N}$ e tal que $k \leq E_{1}<E_{2}<\ldots<E_{k}$.

\section{Demonstração:}

1) Vamos dividir esta demonstração em quatro partes:

a) $\left\|e_{n}\right\|=1, \forall n$.

De fato, $\left\|e_{n}\right\|_{0} \leq\left\|e_{n}\right\|_{1} \leq \ldots$ e como $\left\|e_{n}\right\|_{0}=1$ temos que $1 \leq\left\|e_{n}\right\|_{m}, \forall m \geq 0$. Pela segunda propriedade da norma temos que $\left\|e_{n}\right\|_{m} \leq\left\|e_{n}\right\|_{l_{1}}=1, \forall m \geq 0$.

Logo $\left\|e_{n}\right\|_{m}=1 . \forall m \geq 0$ e portanto $\left\|e_{n}\right\|=\lim _{m \rightarrow+\infty}\left\|e_{n}\right\|_{m}=1, \forall n$.

b) $\left(e_{n}\right)_{n}$ é sequència básica.

Pelo Teorema 0.3 é suficiente mostrarmos que

$$
\left\|\sum_{n=1}^{l} a_{n} e_{n}\right\| \leq\left\|\sum_{n=1}^{j} a_{n} e_{n}\right\|
$$

para qualquer sequência de escalares $\left(a_{n}\right)_{n}$ e inteiros $l<j$.

$\left\|\sum_{n=1}^{l} a_{n} e_{n}\right\|_{0}=\max _{1 \leq n \leq l}\left|a_{n}\right| \leq \max _{1 \leq n \leq j}\left|a_{n}\right|=\left\|\sum_{n=1}^{j} a_{n} e_{n}\right\|_{0}$.

Suponhamos que $\left\|\sum_{n=1}^{l} a_{n} e_{n}\right\|_{m} \leq\left\|\sum_{n=1}^{j} a_{n} e_{n}\right\|_{m}, \forall\left(a_{n}\right)_{n}$ e $l<j$. Vamos provar que $\left\|\sum_{n=1}^{l} a_{n} e_{n}\right\|_{m+1} \leq\left\|\sum_{n=1}^{j} a_{n} e_{n}\right\|_{m+1}$.

$\left\|\sum_{n=1}^{l} a_{n} e_{n}\right\|_{m+1}=\max \left\{\left\|\sum_{n=1}^{l} a_{n} e_{n}\right\|_{m}, \frac{1}{2} \max \left[\sum_{j=1}^{k}\left\|E_{j}\left(\sum_{n=1}^{l} a_{n} e_{n}\right)\right\|_{m}\right]\right\} \leq \max \left\{\left\|\sum_{n=1}^{j} a_{n} e_{n}\right\|_{m}\right.$, $\left.\frac{1}{2} \max \left[\sum_{j=1}^{k}\left\|E_{j}\left(\sum_{n=1}^{j} a_{n} e_{n}\right)\right\|_{m}\right]\right\}=\left\|\sum_{n=1}^{j} a_{n} e_{n}\right\|_{m+1}$ pela hipótese de indução. 
Portanto $\left\|\sum_{n=1}^{l} a_{n} e_{n}\right\|_{m} \leq\left\|\sum_{n=1}^{j} a_{n} e_{n}\right\|_{m}, \forall m, \forall\left(a_{n}\right)_{n}$ e $l<j$. Assim $\lim _{m \rightarrow+\infty}\left\|\sum_{n=1}^{l} a_{n} e_{n}\right\|_{m} \leq$ $\lim _{m \rightarrow+\infty}\left\|\sum_{n=1}^{j} a_{n} e_{n}\right\|_{m}$, ou seja, $\left\|\sum_{n=1}^{l} a_{n} e_{n}\right\| \leq\left\|\sum_{n=1}^{j} a_{n} e_{n}\right\|$ para qualquer $\left(a_{n}\right)_{n}$ e $l<j$.

c) $\left(e_{n}\right)_{n}$ gera $\mathrm{T}$.

Demonstração análoga a feita para o espaço de Schreier.

d) $\left(e_{n}\right)_{n}$ é 1-incondicional para T.

Pelo Teorema 0.5 , basta verificarmos que

$$
\left\|\sum_{n \in A} a_{n} e_{n}\right\| \leq\left\|\sum_{n \in B} a_{n} e_{n}\right\|
$$

onde $A$ e $B$ são subconjuntos finitos dos naturais com $A \subset B$.

$\left\|\sum_{n \in A} a_{n} e_{n}\right\|_{0}=\max _{n \in A}\left|a_{n}\right| \leq \max _{n \in B}\left|a_{n}\right|=\left\|\sum_{n \in B} a_{n} e_{n}\right\|_{0}$.

Suponhamos que $\left\|\sum_{n \in A} a_{n} e_{n}\right\|_{m} \leq\left\|\sum_{n \in B} a_{n} e_{n}\right\|_{m}$, onde $A$ e $B$ são subconjuntos finitos dos naturais com $A \subset B$. Vamos provar que $\left\|\sum_{n \in A} a_{n} e_{n}\right\|_{m+1} \leq\left\|\sum_{n \in B} a_{n} e_{n}\right\|_{m+1}$.

$\left\|\sum_{n \in A} a_{n} e_{n}\right\|_{m+1}=\max \left\{\left\|\sum_{n \in A} a_{n} e_{n}\right\|_{m}, \frac{1}{2} \max \left[\sum_{j=1}^{k}\left\|E_{j}\left(\sum_{n \in A} a_{n} e_{n}\right)\right\|_{m}\right]\right\} \leq \max \left\{\left\|\sum_{n \in B} a_{n} e_{n}\right\|_{m}\right.$, $\left.\frac{1}{2} \max \left[\sum_{j=1}^{k}\left\|E_{j}\left(\sum_{n \in B} a_{n} e_{n}\right)\right\|_{m}\right]\right\}=\left\|\sum_{n \in B} a_{n} e_{n}\right\|_{m+1}$ pela hipótese de indução.

Portanto $\left\|\sum_{n \in A} a_{n} e_{n}\right\|_{m} \leq\left\|\sum_{n \in B} a_{n} e_{n}\right\|_{m}, \forall m, A \subset B$. Assim $\lim _{m \rightarrow+\infty}\left\|\sum_{n \in A} a_{n} e_{n}\right\|_{m} \leq$ $\leq \lim _{m \rightarrow+\infty}\left\|\sum_{n \in B} a_{n} e_{n}\right\|_{m}$, ou seja, $\left\|\sum_{n \in A} a_{n} e_{n}\right\| \leq\left\|\sum_{n \in B} a_{n} e_{n}\right\|$, onde $A \subset B$.

Portanto $\left(e_{n}\right)_{n}$ é base de Schauder normalizada 1-incondicional para T.

2) Sejam $x \in c_{00}$ e $m \geq 0$. Suponhamos que $\|x\|_{m+1}>\sup \left\{\frac{1}{2} \sum_{j=1}^{k}\left\|E_{j} x\right\|_{m}: k \leq\right.$ $\left.E_{1}<E_{2}<\ldots<E_{k}, k=1,2, \ldots\right\}$.

Pela definição de $\|x\|_{m+1}$ temos que $\|x\|_{m+1}=\|x\|_{m}$. Logo $\|x\|_{m}>$ $\sup \left\{\frac{1}{2} \sum_{j=1}^{k}\left\|E_{j} x\right\|_{m}: k \leq E_{1}<\ldots<E_{k}, k=1,2, \ldots\right\} \geq \sup \left\{\frac{1}{2} \sum_{j=1}^{k}\left\|E_{j} x\right\|_{m-1}: k \leq\right.$ 
$\left.E_{1}<E_{2}<\ldots<E_{k}, k=1.2, \ldots\right\}$.

Pela definição de $\|x\|_{m}$ temos que $\|x\|_{m}=\|x\|_{m-1}$. Continuando este raciocínio teremos que $\|x\|_{m+1}=\|x\|_{0}$.

3) Vamos verificar primeiro que essa igualdade vale para $x \in c_{00}$.

Para $x \in c_{00}$ temos que $\|x\|_{m+1}=\max \left\{\|x\|_{0}, \sup \left\{\frac{1}{2} \sum_{j=1}^{k}\left\|E_{j} x\right\|_{m}: k \leq E_{1}<E_{2}<\right.\right.$ $\left.\left.\ldots<E_{k}, k=1,2, \ldots\right\}\right\}, \forall m \geq 0$.

De fato, se $\|x\|_{m+1}>\sup \left\{\frac{1}{2} \sum_{j=1}^{k}\left\|E_{j} x\right\|_{m}: k \leq E_{1}<E_{2}<\ldots<E_{k}, k=1,2, \ldots\right\}$ então pelo ítem anterior temos que $\|x\|_{m+1}=\|x\|_{0}$.

Se $\|x\|_{m+1}>\|x\|_{0}$, então necessariamente $\|x\|_{m+1}=\sup \left\{\frac{1}{2} \sum_{j=1}^{k}\left\|E_{j} x\right\|_{m}: k \leq E_{1}<\right.$ $\left.E_{2}<\ldots<E_{k}, k=1,2, \ldots\right\}$ pois caso contrário teríamos $\|x\|_{m+1}>\sup \left\{\frac{1}{2} \sum_{j=1}^{k}\left\|E_{j} x\right\|_{m}\right.$ : $\left.k \leq E_{1}<E_{2}<\ldots<E_{k}, k=1,2, \ldots\right\}$, mas como antes temos que isso implica que $\|x\|_{m+1}=\|x\|_{0}$ : absurdo.

Como a função máximo é continua passando o limite temos:

$\lim _{m \rightarrow+\infty}\|x\|_{m+1}=\lim _{m \rightarrow+\infty} \max \left\{\|x\|_{0}, \sup \left\{\frac{1}{2} \sum_{j=1}^{k}\left\|E_{j} x\right\|_{m}: k \leq E_{1}<\ldots<E_{k}, k=1, ..\right\}\right\}$.

Pela definição de \|.|| temos:

$\|x\|=\max \left\{\|x\|_{0}, \sup \lim _{m \rightarrow+\infty}\left\{\frac{1}{2} \sum_{j=1}^{k}\left\|E_{j} x\right\|_{m}: k \leq E_{1}<E_{2}<\ldots<E_{k}, k=1,2, \ldots\right\}\right\}$.

Portanto, $\|x\|=\max \left\{\|x\|_{0}, \frac{1}{2} \sup \left\{\sum_{j=1}^{k}\left\|E_{j} x\right\|: k \leq E_{1}<E_{2}<\ldots<E_{k}, k=1,2, \ldots\right\}\right\}$, para $x \in c_{00}$.

Seja $x=\sum_{n=1}^{\infty} a_{n} e_{n} \in T$. Vamos fazer agora duas Observações relativas a norma $\|$.$\| .$

Observação 1: $\max _{n}\left|a_{n}\right| \leq\|x\|$.

Existe $\max _{n}\left|a_{n}\right|$ porque $\left(a_{n}\right)_{n} \in c_{0}(\mathbb{N})$. Consideremos $S_{N}=\sum_{i=1}^{N} a_{i} e_{i}$. 
Seja $n_{0} \in \mathbb{N}$ tal que $\max _{n}\left|a_{n}\right|=\left|a_{n_{0}}\right|$. Logo $\forall N, N \geq n_{0}$ temos $\max _{n}\left|a_{n}\right|=\left\|S_{N}\right\|_{0} \leq$ $\left\|S_{N}\right\|$. Fazendo $N \rightarrow+\infty$ temos que $\left\|S_{N}\right\| \rightarrow\|x\|$, portanto $\max _{n}\left|a_{n}\right|^{n} \leq\|x\|$.

Observação 2: $\forall k \in \mathbb{N}$ e $k \leq E_{1}<E_{2}<\ldots<E_{k}$ temos $\frac{1}{2} \sum_{j=1}^{k}\left\|E_{j} x\right\| \leq\|x\|$.

Seja $n_{k}>\max \left(E_{k}\right)$. Logo $\forall N, N \geq n_{k}$ temos $\frac{1}{2} \sum_{j=1}^{k}\left\|E_{j} x\right\| \leq \frac{1}{2} \sum_{j=1}^{k}\left\|E_{j}\left(S_{N}\right)\right\| \leq\left\|S_{N}\right\|$ pelo o que já vimos. Fazendo $N \rightarrow+\infty$ temos que $\left\|S_{N}\right\| \rightarrow\|x\|$, portanto $\frac{1}{2} \sum_{j=1}^{k}\left\|E_{j} x\right\| \leq$ $\|x\|$.

Vamos definir agora sobre $T$ a norma $\||x|\|=\max \left\{\max _{n}\left|a_{n}\right|, \frac{1}{2} \sup \sum_{j=1}^{k}\left\|E_{j} x\right\|: k \leq E_{1}<\ldots<E_{k}, k=1,2, \ldots\right\}, \forall x \in T$.

$(T,\|||\|$.$) é Banach. De fato, seja x^{n}=\left(x_{k}^{n}\right)_{k} \in T$ uma sequência de Cauchy. Portanto dado $\epsilon>0$, existe $n_{0} \in \mathbb{N}$ tal que para $n, m \geq n_{0}$ temos $\left\|\left|x^{n}-x^{m}\right|\right\|<\epsilon$, isto é, $\max \left\{\max _{k}\left|x_{k}^{n}-x_{k}^{m}\right|, \frac{1}{2} \sup \sum_{j=1}^{l}\left\|E_{j}\left(x^{n}-x^{m}\right)\right\|: l \leq E_{1}<E_{2}<\ldots<E_{l}, l=1,2, \ldots\right\}<\epsilon$.

Então, em particular, temos $\left|x_{k}^{n}-x_{k}^{m}\right|<\epsilon \forall k$. Assim para cada $k$ a sequência $\left(x_{k}^{n}\right)_{n}$ é Cauchy em $\mathbb{R}^{n}$, portanto tem um limite que indicaremos por $x_{k}$.

Vamos demonstrar que $x=\left(x_{k}\right)_{k} \in T$ e que $\left\|\left|x^{n}-x\right|\right\| \rightarrow 0$.

1. $\max _{k}\left|x_{k}^{n}-x_{k}^{m}\right|<\epsilon, \forall m, n \geq n_{0}$. Fazendo $m \rightarrow+\infty$ temos $\max _{k}\left|x_{k}^{n}-x_{k}\right|<\epsilon, \forall n \geq$ $n_{0}$.

2. $\frac{1}{2} \sup \sum_{j=1}^{l}\left\|E_{j}\left(x^{n}-x^{m}\right)\right\|<\epsilon \forall m, n \geq n_{0}$.

Temos que $\sum_{j=1}^{l}\left\|E_{j}\left(x^{n}-x^{m}\right)\right\|=\sum_{j=1}^{l} \lim _{r}\left\|E_{j}\left(x^{n}-x^{m}\right)\right\|_{r}$. Vamos verificar que $\forall r, \forall E_{j}$ $\lim _{m \rightarrow+\infty}\left\|E_{j}\left(x^{n}-x^{m}\right)\right\|_{r}=\left\|E_{j}\left(x^{n}-x\right)\right\|_{r}$.

Para $r=0$, $\lim _{m \rightarrow+\infty}\left\|E_{j}\left(x^{n}-x^{m}\right)\right\|_{0}=\lim _{m \rightarrow+\infty} \max _{k \in E_{j}}\left|x_{k}^{n}-x_{k}^{m}\right|=\max _{k \in E_{j}}\left|x_{k}^{n}-x_{k}\right|=\left\|E_{j}\left(x^{n}-x\right)\right\|_{0}$.

Suponhamos que $\left({ }^{*}\right)$ é verdadeiro até $r-1$. Vamos provar que é verdade para $r$. 
$\left\|E_{j}\left(x^{n}-x^{m}\right)\right\|_{r}=\max \left\{\left\|E_{j}\left(x^{n}-x^{m}\right)\right\|_{r-1}, \frac{1}{2} \max \left[\sum_{\theta=1}^{\gamma}\left\|E_{\theta} E_{j}\left(x^{n}-x^{m}\right)\right\|_{r-1}\right]\right\}$

$\lim _{m \rightarrow+\infty}\left\|E_{j}\left(x^{n}-x^{m}\right)\right\|_{r}=\max \left\{\lim _{m \rightarrow+\infty}\left\|E_{j}\left(x^{n}-x^{m}\right)\right\|_{r-1}, \frac{1}{2} \max \left[\sum_{\theta=1}^{\gamma} \lim _{m \rightarrow+\infty}\left\|E_{\theta} E_{j}\left(x^{n}-x^{m}\right)\right\|_{r-1}\right]\right\}$

$\lim _{m \rightarrow+\infty}\left\|E_{j}\left(x^{n}-x^{m}\right)\right\|_{r}=\max \left\{\left\|E_{j}\left(x^{n}-x\right)\right\|_{r-1}, \frac{1}{2} \max \left[\sum_{\theta=1}^{\gamma}\left\|E_{\theta} E_{j}\left(x^{n}-x\right)\right\|_{r-1}\right]\right\}=\| E_{j}\left(x^{n}-\right.$ $x) \|_{r}$.

Logo $\lim _{m \rightarrow+\infty} \sum_{j=1}^{l}\left\|E_{j}\left(x^{n}-x^{m}\right)\right\|=\lim _{m \rightarrow+\infty} \sum_{j=1}^{l} \lim _{r}\left\|E_{j}\left(x^{n}-x^{m}\right)\right\|_{r}=\sum_{j=1}^{l} \lim _{r} \| E_{j}\left(x^{n}-\right.$ $x)\left\|_{r}=\sum_{j=1}^{l}\right\| E_{j}\left(x^{n}-x\right) \|$.

Portanto fazendo $m \rightarrow+\infty$ em $\frac{1}{2} \sup \sum_{j=1}^{l}\left\|E_{j}\left(x^{n}-x^{m}\right)\right\|<\epsilon, \quad \forall m, n \geq n_{0}$ obtemos $\frac{1}{2} \sup \sum_{j=1}^{l}\left\|E_{j}\left(x^{n}-x\right)\right\|<\epsilon \quad \forall n \geq n_{0}$.

$\operatorname{Logo} \max \left\{\max _{k}\left|x_{k}^{n}-x_{k}\right|, \frac{1}{2} \sup \sum_{j=1}^{l}\left\|E_{j}\left(x^{n}-x\right)\right\|\right\}<\epsilon$, ou seja, $\left\|\left|x^{n}-x\right|\right\|<\epsilon \quad \forall n \geq n_{0}$.

Resta mostrar que $x=\left(x_{k}\right)_{k} \in T$, para isto vamos verificar que $P_{i}(x)$ é Cauchy na norma $\|$.$\| , onde P_{i}(x)$ representa a projeção de $x$ no intervalo $[1, i]$.

Seja $\|E(x)\|=\left\|P_{i}(x)-P_{m}(x)\right\|$ onde $E=\{i+1, \ldots . m\}, i<m$. Temos que $\|E(x)\|=\left\|E\left(x-x^{n}\right)+E\left(x^{n}\right)\right\| \leq\left\|E\left(x-x^{n}\right)\right\|+\left\|E\left(x^{n}\right)\right\| \leq 2\left\|\left|x-x^{n}\right|\right\|+\left\|E\left(x^{n}\right)\right\| \leq$ $2\left\|\left|x-x^{n}\right|\right\|+\sum_{l=i+1}^{m}\left|x_{l}^{n}\right|$, pois $E\left(x^{n}\right) \in c_{00} \operatorname{logo}\left\|E\left(x^{n}\right)\right\| \leq\left\|E\left(x^{n}\right)\right\|_{l_{1}}$.

Dado $\epsilon>0$ fixemos $n_{0}, n_{0} \in \mathbb{N}$, tal que para $n \geq n_{0}$ temos $\left\|\left|x-x^{n}\right|\right\|<\frac{\epsilon}{4}$. Então $\left\|P_{i}(x)-P_{m}(x)\right\| \leq 2\left\|\left|x-x^{n_{0}}\right|\right\|+\sum_{l=i}^{m}\left|x_{l}^{n_{0}}\right|$. Dado o mesmo $\epsilon>0, \exists k_{0} \in \mathbb{N}$ tal que $i, m \geq k_{0}$ temos $\sum_{l=i+1}^{m}\left|x_{l}^{n_{0}}\right|<\frac{\epsilon}{2}$. Logo $\left\|P_{i}(x)-P_{m}(x)\right\|<\epsilon \forall i, m \geq k_{0}$. Portanto $\left(P_{i}(x)\right)_{i}$ é Cauchy em $(T,\|\cdot\|), \operatorname{assim}\left(P_{i}(x)\right)_{i}$ é convergente em $(T,\|\cdot\|)$.

Então $\left(P_{i}(x)\right)_{i} \stackrel{i \rightarrow+\infty}{\rightarrow} y \in T$, logo as coordenadas de $y$ coincidem com as coordenadas de $x, \operatorname{assim} y=x$ e como $y \in T$ temos que $x \in T$. 
Como $x \in T$ e $\left\|\left|x-x^{n}\right|\right\|<\epsilon, \forall n \geq n_{0}$, temos que $x^{n}=\left(x_{k}^{n}\right)_{k}$ é convergente para um elemento de $T$, sendo assim $(T,\||\cdot|\|)$ é Banach.

Vamos verificar que $P_{n}(x) \stackrel{n \rightarrow+\infty}{\rightarrow} x$ na norma $\|$.$\| e que P_{n}(x) \stackrel{n \rightarrow+\infty}{\rightarrow} x$ na norma $\|||$.$\| .$

Como $P_{n}(x) \rightarrow x$ em $(T,\|\cdot\|)$ temos que $\left\|P_{n}(x)\right\| \rightarrow\|x\|$. Pelas Observações 1 e 2 temos $\left\|\left|P_{n}(x)-x\right|\right\| \leq\left\|P_{n}(x)-x\right\| \rightarrow 0$.

$P_{n}(x) \in c_{00}$ e como foi visto anteriormente sabemos que $\|x\|=\max \left\{\max _{n}\left|a_{n}\right|, \frac{1}{2} \sup \sum_{j=1}^{k}\left\|E_{j} x\right\|, k \leq E_{1}<E_{2}<\ldots<E_{k}\right\}=\||x|\|, \forall x \in c_{00}$.

Portanto $\left\|P_{n}(x)\right\|=\left\|\left|P_{n}(x)\right|\right\|$, mas como $\left\|P_{n}(x)\right\| \rightarrow\|x\|$ e $\left\|\left|P_{n}(x)\right|\right\| \rightarrow\||x|\|$ temos que $\|x\|=\||x|\|, \forall x \in T$.

Com isso verificamos que:

$\|x\|=\max \left\{\max _{n}\left|a_{n}\right|, \frac{1}{2} \sup \sum_{j=1}^{k}\left\|E_{j} x\right\|, k \leq E_{1}<E_{2}<\ldots<E_{k}\right\}$, para qualquer $x \in T$.

Teorema $1.12 l_{1}(\mathbb{N}) \nrightarrow T$.

\section{Demonstração:}

Suponhamos que $l_{1}(\mathbb{N}) \hookrightarrow T$. Logo pelo Teorema 0.14 , com $\delta=\frac{1}{9}$, existe uma base de blocos normalizada $\left(y_{i-1}\right)_{i}$ de $\left(e_{n}\right)_{n}$ tal que para toda escolha de escalares $\left(b_{i-1}\right)_{i}$,

$$
\frac{8}{9} \sum_{i=0}^{\infty}\left|b_{i}\right| \leq\left\|\sum_{i=0}^{\infty} b_{i} y_{i}\right\| \leq \sum_{i=0}^{\infty}\left|b_{i}\right|
$$

Disto segue que $\left\|y_{0}+\frac{1}{r} \sum_{i=1}^{r} y_{i}\right\| \geq \frac{8}{9} \sum_{i=0}^{r}\left|b_{i}\right|$, onde $b_{0}=1$ e $b_{i}=\frac{1}{r}, \forall i, 1 \leq i \leq r$ e como $\sum_{i=0}^{r}\left|b_{i}\right|=2$ temos $\left\|y_{0}+\frac{1}{r} \sum_{i=1}^{r} y_{i}\right\| \geq \frac{16}{9}$, para todo $r=1,2, \ldots$

Agora consideremos $k \leq E_{1}<E_{2}<\ldots<E_{k}$ e $n_{0}=\max \operatorname{supp}\left(y_{0}\right)$.

Se $k>n_{0}$, então

$\sum_{j=1}^{k}\left\|E_{j}\left(y_{0}+\frac{1}{r} \sum_{i=1}^{r} y_{i}\right)\right\|=\sum_{j=1}^{k}\left\|E_{j}\left(\frac{1}{r} \sum_{i=1}^{r} y_{i}\right)\right\| \leq \sup \sum_{j=1}^{k}\left\|E_{j}\left(\frac{1}{r} \sum_{i=1}^{r} y_{i}\right)\right\| \leq$ 
$2\left\|\frac{1}{r} \sum_{i=1}^{r} y_{i}\right\| \leq \frac{2}{r} \sum_{i=1}^{r}\left\|y_{i}\right\|=2 . \log \frac{1}{2} \sup \sum_{j=1}^{k}\left\|E_{j}\left(y_{0}+\frac{1}{r} \sum_{i=1}^{r} y_{i}\right)\right\| \leq 1$

Se $k \leq n_{0}$, consideremos os conjuntos:

$A=\left\{i:\left\|E_{j} y_{i}\right\| \neq 0\right.$ para pelo menos 2 valores de $\left.j\right\}$,

$B=\left\{i:\left\|E_{j} y_{i}\right\| \neq 0\right.$ para no máximo 1 valor de $\left.j\right\}$.

Como $A$ tem no máximo $k$ elementos, temos

$\sum_{j=1}^{k}\left\|E_{j}\left(y_{0}+\frac{1}{r} \sum_{i=1}^{r} y_{i}\right)\right\|=\sum_{j=1}^{k}\left\|E_{j} y_{0}+\frac{1}{r} \sum_{i=1}^{r} E_{j} y_{i}\right\| \leq \sum_{j=1}^{k}\left\|E_{j} y_{0}\right\|+\sum_{j=1}^{k}\left\|\frac{1}{r} \sum_{i=1}^{r} E_{j} y_{i}\right\| \leq$

$\sup \sum_{j=1}^{k}\left\|E_{j} y_{0}\right\|+\frac{1}{r}\left(\sum_{j=1}^{k} \sum_{i=1}^{r}\left\|E_{j} y_{i}\right\|\right) \leq 2\left\|y_{0}\right\|+\frac{1}{r}\left(\sum_{i=1}^{r} \sum_{j=1}^{k}\left\|E_{j} y_{i}\right\|\right)=2\left\|y_{0}\right\|+$

$\frac{1}{r}\left(\left(\sum_{i \in A} \sum_{i \in B}\right) \sum_{j=1}^{k}\left\|E_{j} y_{i}\right\|\right)=2\left\|y_{0}\right\|+\frac{1}{r}\left[\sum_{i \in A} \sum_{j=1}^{k}\left\|E_{j} y_{i}\right\|+\sum_{i \in B} \sum_{j=1}^{k}\left\|E_{j} y_{i}\right\|\right]=2\left\|y_{0}\right\|+$

$\frac{1}{r} \sum_{i \in A} \sum_{j=1}^{k}\left\|E_{j} y_{i}\right\|+\frac{1}{r} \sum_{i \in B} \sum_{j=1}^{k}\left\|E_{j} y_{i}\right\| \leq 2\left\|y_{0}\right\|+\frac{1}{r} \sum_{i \in A} \sup \sum_{j=1}^{k}\left\|E_{j} y_{i}\right\|+\frac{1}{r} \sum_{i \in B} \sum_{j=1}^{k}\left\|E_{j} y_{i}\right\| \leq$

$2\left\|y_{0}\right\|+\frac{2}{r} \sum_{i \in A}\left\|y_{i}\right\|+\frac{1}{r} \sum_{i \in B} \sum_{j=1}^{k}\left\|E_{j} y_{i}\right\| \leq 2+\frac{2|A|}{r}+\frac{|B|}{r}$, pois pela definição do conjunto $B$, para cada $i$, no máximo a norma de uma projeção $E_{j}$ é diferente de zero e $\left\|E_{j} y_{i}\right\| \leq$ $\left\|y_{i}\right\|=1, \log 0 \sum_{i \in B} \sum_{j=1}^{k}\left\|E_{j} y_{i}\right\| \leq|B|$. Continuando temos que

$2+\frac{2|A|}{r}+\frac{|B|}{r} \leq 2+\frac{2 k}{r}+\frac{r-k}{r}=2+\frac{2 k}{r}+1-\frac{k}{r}=3+\frac{k}{r} \leq 3+\frac{n_{0}}{r}$.

Se $r \geq 2 n_{0}$ então $\frac{1}{r} \leq \frac{1}{2 n_{0}}$ e teremos $3+\frac{n_{0}}{r} \leq 3+\frac{n_{0}}{2 n_{0}}=\frac{7}{2}$. Então

$\sum_{j=1}^{k}\left\|E_{j}\left(y_{0}+\frac{1}{r} \sum_{i=1}^{r} y_{i}\right)\right\| \leq \frac{7}{2}, \log \frac{1}{2} \sup \sum_{j=1}^{k}\left\|E_{j}\left(y_{0}+\frac{1}{r} \sum_{i=1}^{r} y_{i}\right)\right\| \leq \frac{7}{4}$.

Pelo ítem 3 da Proposição 1.11 temos que $\left\|y_{i}\right\|_{0} \leq\left\|y_{i}\right\|=1$, para cada $i$. Logo cada coordenada de $y_{i}$ tem módulo no máximo 1 . Como os $y_{i^{\prime} s}$ são consecutivos segue que $\left\|y_{0}+\frac{1}{r} \sum_{i=1}^{r} y_{i}\right\|_{0} \leq 1$.

Por definição $\left\|y_{0}+\frac{1}{r} \sum_{i=1}^{r} y_{i}\right\|=\max \left\{\left\|y_{0}+\frac{1}{r} \sum_{i=1}^{r} y_{i}\right\|_{0}, \frac{1}{2} \sup \sum_{j=1}^{k}\left\|E_{j}\left(y_{0}+\frac{1}{r} \sum_{i=1}^{r} y_{i}\right)\right\|\right\}$ e por (2), (3), e (4) temos $\left\|y_{0}+\frac{1}{r} \sum_{i=1}^{r} y_{i}\right\| \leq \frac{7}{4}$ o que contradiz (1). 
Teorema $1.13 c_{0}(\mathbb{N}) \nrightarrow T$ e $l_{p}(\mathbb{N}) \hookrightarrow T, \forall 1<p<\infty$.

\section{Demonstração:}

Suponhamos, por absurdo, que exista $\left(y_{n}\right)_{n}$ uma sequência básica em $T$ equivalente a base canônica de $c_{0}(\mathbb{N})$ ou de $l_{p}(\mathbb{N}), 1<p<\infty$. Logo $\left(y_{n}\right)_{n}$ é fraca nula, e é limitada inferiormente e superiormente.

Pelo Teorema $0.9\left(y_{n}\right)_{n}$ deve ter uma subsequência que é uma sequência básica e essa sequência básica é equivalente a uma base de blocos $\left(x_{n}\right)_{n}$ de $\left(e_{n}\right)_{n}$.

Passando para uma subsequência podemos assumir que $\operatorname{supp}\left(x_{i}\right)>i, \forall i$.

Como as bases canônicas de $c_{0}(\mathbb{N})$ e $l_{p}(\mathbb{N}), 1<p<\infty$. tem a propriedade de serem equivalentes a qualquer subsequência estritamente crescente dessas bases, pois

1) $\operatorname{Em} l_{p}(\mathbb{N})$

Sejam $n_{1}<n_{2}<n_{3}<\ldots$ e $\left(a_{n}\right)_{n}$

$\sum_{i=1}^{\infty} a_{i} e_{i}$ é convergente $\Longleftrightarrow \sum_{i=1}^{\infty}\left|a_{i}\right|<\infty \Longleftrightarrow \sum_{i=1}^{\infty} a_{i} e_{n_{i}}$ é convergente.

2) $\operatorname{Em} c_{0}(\mathbb{N})$

$\sum_{i=1}^{\infty} a_{i} e_{i}$ é convergente $\Longleftrightarrow \lim _{n \rightarrow+\infty} a_{n}=0 \Longleftrightarrow \sum_{i=1}^{\infty} a_{i} e_{n_{i}}$ é convergente.

Segue que existe $M>0$ tal que para qualquer $m \in \mathbb{N}$, quaisquer escalares $a_{1}, \ldots a_{m}$ e qualquer escolha de $n_{1}<n_{2}<\ldots<n_{m}$, temos $\left\|\sum_{k=1}^{m} a_{k} y_{k}\right\| \geq M\left\|\sum_{k=1}^{m} a_{k} y_{n_{k}}\right\|$.

Logo $\left\|\sum_{k=1}^{m} a_{k} y_{k}\right\| \geq M\left\|\sum_{k=1}^{m} a_{k} y_{n_{m-1+k}}\right\| \geq M M^{\prime}\left\|\sum_{k=1}^{m} a_{k} x_{m-1+k}\right\| \geq$ $\geq \frac{1}{2} M M^{\prime} \sum_{j=1}^{l}\left\|E_{j}\left(\sum_{k=1}^{m} a_{k} x_{m-1+k}\right)\right\|=\frac{1}{2} M M^{\prime} \sum_{j=1}^{l}\left\|\sum_{k=1}^{m} a_{k} E_{j}\left(x_{m-1+k}\right)\right\|$, onde $M^{\prime}$ é a constante de equivalência de $\left(x_{n}\right)_{n}$ e $\left(y_{n_{i}}\right)_{i}$.

Tomemos $l=m$ e os intervalos $E_{j}=\operatorname{supp}\left(x_{m-1+j}\right), \forall j, 1 \leq j \leq m$. Logo $m \leq$ $E_{1}<\ldots<E_{m}$ e $\left\|\sum_{k=1}^{m} a_{k} y_{k}\right\| \geq \frac{1}{2} M M^{\prime} N \sum_{k=1}^{m}\left|a_{k}\right|$, onde $N$ é a constante que limita $\left(x_{n}\right)_{n}$ superiormente.

Portanto $\frac{1}{2} M M^{\prime} N \sum_{k=1}^{m}\left|a_{k}\right| \leq\left\|\sum_{k=1}^{m} a_{k} y_{k}\right\| \leq M^{\prime \prime} \sum_{k=1}^{m}\left|a_{k}\right|$, onde $M^{\prime \prime}$ é a constante que 
limita $\left\|y_{k}\right\|, k \in \mathbb{N}$. Logo $\left(y_{n}\right)_{n}$ é equivalente a base canônica de $l_{1}(\mathbb{N})$; absurdo, pois $l_{1}(\mathbb{N}) \nrightarrow T$.

Observação $1.14 T$ é reflexivo.

De fato, como $T$ tem base incondicional, pelo Teorema acima e o Teorema 0.13 segue que $T$ é reflexivo. 


\section{Capítulo 2}

\section{Funções}

Antes de apresentarmos o espaço de Banach de Thomas Schlumprecht (capítulo 3) e o espaço de Gowers e Maurey (capítulo 4) será necessário estudarmos algumas propriedades de funções reais a valores reais.

Essencialmente destacamos um subconjunto $\mathcal{F}$ destas funções contendo as funções $f(x)=\log _{2}(x+1)$ e $\sqrt{f(x)}$. A função $f(x)$ será usada tanto na definição da norma do espaço de Thomas Schlumprecht como na definição da norma do espaço de Gowers e Maurey.

Na observação 2.10 construiremos funções que serão utilizadas em Lemas dos capítulos 3 e 4 .

Este capítulo está baseado em [11] e começaremos definindo $\mathcal{F}$.

Denotaremos por $\mathcal{F}$ o conjunto das funções $f:[1,+\infty) \rightarrow[1,+\infty)$, satisfazendo as seguintes condições:

1) $f(1)=1$ e $f(x)<x$ para todo $x>1$.

2) $f$ é estritamente crescente e $\lim _{x \rightarrow+\infty} f(x)=+\infty$.

3) $\lim _{x \rightarrow+\infty} x^{-q} f(x)=0$ para todo $q>0$.

4) A função $\frac{x}{f(x)}$ é concava.

5) $f(x y) \leq f(x) f(y)$ para todo $x, y \geq 1$.

Observação 2.1 A função $f(x)=\log _{2}(x+1) \in \mathcal{F}$. 
De fato,

1) $f(1)=\log _{2}(1+1)=\log _{2}(2)=1$.

Verificaremos agora que $f(x)=\log _{2}(x+1)<x, \forall x>1$.

Seja $d(x)=x-\log _{2}(x+1)$, então $d(1)=1-1=0$.

Basta mostrarmos que $d$ é crescente e teremos que $\log _{2}(x+1)<x$.

$d^{\prime}(x)=1-\log _{2} e \frac{1}{x+1}$.

$d^{\prime}(x)>0, \forall x>1 \Longleftrightarrow 1>\log _{2} e \frac{1}{x+1}, \forall x>1$, ou seja, $x+1>\log _{2} e, \forall x>1$. O que é verdade porque $\log _{2} e<2$, pois $2^{2}>e$.

2) $f$ é estritamente crescente.

De fato, sejam $x<y, f(x)=\log _{2}(x+1)$ e $f(y)=\log _{2}(y+1)$.

$\log _{2}(x+1)<\log _{2}(y+1) \Longleftrightarrow x+1<y+1 \Longleftrightarrow x<y$.

Claramente $\lim _{x \rightarrow+\infty} f(x)=+\infty$.

3) $\lim _{x \rightarrow+\infty} \frac{f(x)}{x^{q}}=\lim _{x \rightarrow+\infty} \frac{\log _{2}(x+1)}{x^{q}}$ que vai tanto no numerador quanto no denominador para $+\infty$. Então vamos usar a regra de L'Hospital.

$\lim _{x \rightarrow+\infty} \frac{\log _{2} e \frac{1}{x+1}}{q x^{q-1}}=\lim _{x \rightarrow+\infty} \frac{1}{q} \log _{2} e \frac{1}{x+1} \frac{1}{x^{q-1}}$.

Quando $q=1$ temos $\lim _{x \rightarrow+\infty} \log _{2} e \frac{1}{x+1}=0$.

Quando $q>1$ temos que $q-1>0$ então $\lim _{x \rightarrow+\infty} \frac{1}{q} \log _{2} e \frac{1}{x+1} \frac{1}{x^{q-1}}=0$.

Quando $q<1$ temos $\lim _{x \rightarrow+\infty} \frac{1}{q} \log _{2} e \frac{x^{1-q}}{x+1}$ que vai para o infinito tanto no numerador quanto no denominador, então vamos usar novamente a regra de L'Hospital.

$\lim _{x \rightarrow+\infty} \frac{1}{q} \log _{2} e \frac{(1-q) x^{-q}}{1}=\lim _{x \rightarrow+\infty} \frac{1}{q} \log _{2} e \frac{(1-q)}{x^{q}}=0$.

4) Vamos mostrar que $D(x)=\frac{x}{\log _{2}(x+1)}$ é concava e para isto demonstraremos que $D^{\prime \prime}(x)<0, \forall x \geq 1$.

$$
D^{\prime}(x)=\frac{\log _{2}(x+1)-x\left(\frac{1}{x+1} \log _{2} e\right)}{\left(\log _{2}(x+1)\right)^{2}}
$$




$$
D^{n}(x)=\underbrace{\frac{\log _{2} e \log _{2}(x+1)}{(x+1)\left(\log _{2}(x+1)\right)^{4}}}_{>0}\left(\frac{x}{x+1} \log _{2}(x+1)-2 \log _{2}(x+1)+\frac{2 x}{x+1} \log _{2} e\right)
$$

Seja $F(x)=\frac{x}{x+1} \log _{2}(x+1)-2 \log _{2}(x+1)+\frac{2 x}{x+1} \log _{2} e$.

$F(1)=\frac{1}{2}-2+\log _{2} e<0 \Longleftrightarrow \log _{2} e<\frac{3}{2} \Longleftrightarrow e<2^{3 / 2}$, o que é verdade.

$$
F^{\prime}(x)=\frac{\log _{2}(x+1)-x \log _{2} e}{(x+1)^{2}}=\underbrace{\frac{1}{(x+1)^{2}}}_{>0}\left(\log _{2}(x+1)-x \log _{2} e\right) .
$$

Agora vamos considerar $G(x)=\log _{2}(x+1)-x \log _{2} e$.

$G(1)=1-\log _{2} e<0 \Longleftrightarrow \log _{2} e>1$, o que é verdade.

$G^{\prime}(x)=\log _{2} e \frac{1}{x+1}-\log _{2} e=\log _{2} e\left(\frac{1}{x+1}-1\right)=\log _{2} e\left(\frac{-x}{x+1}\right)<0$, pois $\frac{\log _{2} e}{x+1}>0$ e $-x<0$.

Assim temos que $G$ é estritamente decrescente e como $G(1)<0$ temos que $G$ é negativa, $\forall x \geq 1$.

Com isto temos que $F^{\prime}(x)<0$. Portanto $F$ é estritamente decrescente e como $F(1)<0$ temos que $F$ é negativa, $\forall x \geq 1$.

Logo $D^{\prime \prime}(x)<0, \forall x \geq 1$ e portanto $D$ é concava.

5)Vamos mostrar que $\log _{2}(x y+1) \leq \log _{2}(x+1) \log _{2}(y+1), \forall x, y \geq 1$

Fixemos $y$ e consideremos a função,

$D(x)=\log _{2}(x y+1)-\log _{2}(x+1) \log _{2}(y+1)$. Temos que mostrar que $D(x) \leq 0, \forall x \geq 1$. $D(1)=\log _{2}(y+1)-\log _{2}(y+1)=0$.

$D^{\prime}(x)=\log _{2} e \frac{y}{x y+1}-\log _{2} e \frac{1}{x+1} \log _{2}(y+1)=\underbrace{\log _{2} e}_{>0}\left(\frac{y}{x y+1}-\frac{1}{x+1} \log _{2}(y+1)\right)$.

$D^{\prime}(x) \leq 0, \forall x \geq 1 \Longleftrightarrow \frac{y}{x y+1}-\frac{1}{x+1} \log _{2}(y+1) \leq 0, \forall x \geq 1$

$\frac{y}{x y+1}-\frac{1}{x+1} \log _{2}(y+1) \leq 0 \Longleftrightarrow \frac{y x+y}{x y+1} \leq \log _{2}(y+1) \Longleftrightarrow \frac{y x+1-1+y}{x y+1} \leq \log _{2}(y+1) \Longleftrightarrow$ $1+\frac{y-1}{x y+1}-\log _{2}(y+1) \leq 0$.

Consideremos a função $F(x)=1+\frac{y-1}{x y+1}-\log _{2}(y+1)$. Vamos verificar que $F$ é negativa. 
$F(1)=1+\frac{y-1}{y+1}-\log _{2}(y+1)$

Precisamos verificar que a função $H(y)=1+\frac{y-1}{y+1}-\log _{2}(y+1) \leq 0, \forall y \geq 1$.

$H(1)=1-\log _{2} 2=1-1=0$

$H^{\prime}(y)=\frac{(y+1)-(y-1)}{(y+1)^{2}}-\log _{2} e \frac{1}{(y+1)}=\underbrace{\frac{1}{(y+1)}}_{>0}\left(\frac{2}{y+1}-\log _{2} e\right)$.

$H^{\prime}(y) \leq 0 \Longleftrightarrow \frac{2}{y+1}-\log _{2} e \leq 0 \Longleftrightarrow \frac{2}{y+1} \leq \log _{2} e$.

Sabemos que $\frac{2}{y+1} \leq 1<\log _{2} e, \log H^{\prime}(y) \leq 0$ e assim $H$ é decrescente, e como $H(1)=0$ segue $H(y) \leq 0, \forall y \geq 1$. Sendo assim temos que $F(1) \leq 0$.

$F^{\prime}(x)=\frac{-(y-1) y}{(x y+1)^{2}} \leq 0$. Portanto $F$ é decrescente e $F(x) \leq 0, \forall x \geq 1$.

Como $F(x) \leq 0$ temos que $D^{\prime}(x) \leq 0$, logo $D$ é decrescente, e como $D(1)=0$ segue que $D \leq 0, \forall x>1$.

Desde que $f(x)=\log _{2}(x+1)$ satisfaz 1),2), 3). 4), e 5) concluímos que $f \in \mathcal{F}$.

Observação 2.2 A função $\sqrt{f(x)} \in \mathcal{F}$.

De fato,

1) Claramente $\sqrt{f(1)}=1$.

Temos que $f(x)<x$ para todo $x>1$, logo $\sqrt{f(x)}<\sqrt{x} \leq x$, para todo $x>1$ pois $\sqrt{x} \leq x \Longleftrightarrow x \leq x^{2} \Longleftrightarrow 1 \leq x$ que é o caso em que estamos trabalhando.

2) Como $f$ é estritamente crescente e tende ao infinito temos que $\sqrt{f(x)}$ também satisfaz essas duas propriedades.

3) $\lim _{x \rightarrow+\infty} \frac{\sqrt{f(x)}}{x^{q}}=\lim _{x \rightarrow+\infty}\left(\frac{f(x)}{x^{2 q}}\right)^{1 / 2}=0, \forall q>0$, pois $\lim _{x \rightarrow+\infty} \frac{f(x)}{x^{2 q}}=0, \forall q>0$.

4) Vamos verificar que $F(x)=\frac{x}{\sqrt{\log _{2}(x+1)}}$ é concava, para isto demonstraremos que $F^{\prime \prime}(x)<0, \forall x \geq 1$.

$F^{\prime}(x)=\frac{\sqrt{\log _{2}(x+1)}-x\left(\frac{\log _{2} e}{2 \sqrt{\log _{2}(x+1)(x+1)}}\right)}{\log _{2}(x+1)}=\frac{2(x+1) \log _{2}(x+1)-x \log _{2} e}{2(x+1)\left(\log _{2}(x+1)\right)^{3 / 2}}$ 


$$
\begin{aligned}
& F^{\prime \prime}(x)=\frac{-4(x+1)\left(\log _{2}(x+1)\right)^{3 / 2} \log _{2} e+2 x\left(\log _{2}(x+1)\right)^{3 / 2} \log _{2} e+3 x\left(\log _{2}(x+1)\right)^{3 / 2}\left(\log _{2} e\right)^{2}}{\left(2(x+1)\left(\log _{2}(x+1)\right)^{3 / 2}\right)^{2}} \\
& F^{\prime \prime}(x)=\underbrace{\frac{\log _{2} e\left(\log _{2}(x+1)\right)^{1 / 2}}{2(x+1)\left(\log _{2}(x+1)\right)^{3 / 2}}}_{>0}\left(-2 x \log _{2}(x+1)-4 \log _{2}(x+1)+3 x \log _{2} e\right)
\end{aligned}
$$

Consideremos $D(x)=-2 x \log _{2}(x+1)-4 \log _{2}(x+1)+3 x \log _{2} e$.

$D(1)=-2-4+3 \log _{2} e<0$, pois $3 \log _{2} e<6$.

$D^{\prime}(x)=-2 \log _{2}(x+1)-\frac{2 x \log _{2} e}{x+1}-\frac{4 \log _{2} e}{(x+1)^{2}}+3 \log _{2} e$.

$D^{\prime}(x)=\underbrace{\frac{1}{x+1}}_{>0}\left(-2 x \log _{2}(x+1)-2 \log _{2}(x+1)+x \log _{2} e-\log _{2} e\right)$.

Consideremos $H(x)=-2 x \log _{2}(x+1)-2 \log _{2}(x+1)+x \log _{2} e-\log _{2} e$.

$H(1)=-2-2=-4$.

$H^{\prime}(x)=-2 \log _{2}(x+1)-\frac{2 x \log _{2} e}{x+1}-\frac{2 \log _{2} e}{x+1}+\log _{2} e$.

$H^{\prime}(x)=\frac{-2 x \log _{2}(x+1)-2 \log _{2}(x+1)-x \log _{2} e-\log _{2} e}{x+1}$.

Como o numerador da fração é negativo e o denominador é positivo temos que $H^{\prime}(x)<$ 0 . Portanto $H$ é negativa, $\forall x \geq 1$, e consequentemente $D$ é negativa, $\forall x \geq 1$. Logo $F$ " é negativa $\forall x \geq 1$ e assim temos que $F$ é concava.

5) Como $f(x y) \leq f(x) f(y)$ para qualquer $x, y \geq 1$ segue que $\sqrt{f(x y)} \leq \sqrt{f(x) f(y)}=$ $\sqrt{f(x)} \sqrt{f(y)}, \forall x, y \geq 1$.

Por 1), 2), 3), 4) e 5) temos que $\sqrt{f(x)} \in \mathcal{F}$.

Observação $2.3 \frac{x}{f(x)}$ é estritamente crescente.

De fato, seja $x<y, x, y \geq 1$

$\frac{x}{f(x)}<\frac{y}{f(y)} \Longleftrightarrow x f(y)<y f(x) \Longleftrightarrow f(y)<\frac{y}{x} f(x)$, mas $f(y)=f\left(\frac{y}{x} x\right) \leq f\left(\frac{y}{x}\right) f(x)<\frac{y}{x} f(x)$. 
Analogamente temos que $\frac{x}{\sqrt{f(x)}}$ é estritamente crescente.

A seguir introduziremos os conceitos de função submultiplicativa, envoltória submultiplicativa e envoltória concava que serão utilizados para construirmos as funções da Observação 2.10 .

Definição 2.4 Dizemos que uma função $f:[1,+\infty) \rightarrow[1,+\infty)$ é submultiplicativa se $f(x y) \leq f(x) f(y), \forall x, y \geq 1$.

Definição 2.5 Seja $f:[1,+\infty) \rightarrow[1,+\infty)$. A envoltória submultiplicativa de $f$ é dada por $F(x)=\inf \left\{f\left(x_{1}\right) f\left(x_{2}\right) \ldots f\left(x_{k}\right): k \in \mathbb{N}, x_{i} \geq 1, x_{1} \ldots x_{k} \geq x\right\}$.

Estudaremos agora algumas propriedades da envoltória submultiplicativa $F$.

1) $F$ é não decrescente. De fato, se tomarmos $x_{1}<x_{2}$ temos

$F\left(x_{1}\right)=\inf \left\{f\left(x_{11}\right) f\left(x_{12}\right) \ldots f\left(x_{1 k}\right): k \in \mathbb{N}, x_{1 i} \geq 1, x_{11} \ldots x_{1 k} \geq x_{1}\right\}$

$F\left(x_{2}\right)=\inf \left\{f\left(x_{21}\right) f\left(x_{22}\right) \ldots f\left(x_{2 q}\right): q \in \mathbb{N}, x_{2 i} \geq 1, x_{21} \ldots x_{2 q} \geq x_{2}\right\}$

Como $A=\left\{f\left(x_{11}\right) f\left(x_{12}\right) \ldots f\left(x_{1 k}\right): k \in \mathbb{N}, x_{1 i} \geq 1, x_{11} \ldots x_{1 k} \geq x_{1}\right\} \supset\left\{f\left(x_{21}\right) f\left(x_{22}\right) \ldots\right.$ ..f $\left.f\left(x_{2 q}\right): q \in \mathbb{N}, x_{2 i} \geq 1, x_{21} \ldots x_{2 q} \geq x_{2}\right\}=B$, segue que inf $A \leq \inf B$.

2) $F(x) \leq f(x), \forall x \geq 1$.

$F(x)=\inf \left\{f\left(x_{1}\right) f\left(x_{2}\right) \ldots f\left(x_{k}\right): k \in \mathbb{N}, x_{i} \geq 1, x_{1} \ldots x_{k} \geq x\right\}$ e como $x \geq x$ temos que $F(x) \leq f(x)$.

3) $F$ é submultiplicativa.

Sejam $x, y \geq 1, F(x)=\inf \left\{f\left(x_{1}\right) f\left(x_{2}\right) \ldots f\left(x_{k}\right): k \in \mathbb{N}, x_{i} \geq 1, x_{1} \ldots x_{k} \geq x\right\}$ e $F(y)=\inf \left\{f\left(y_{1}\right) f\left(y_{2}\right) \ldots f\left(y_{l}\right): l \in \mathbb{N}, y_{i} \geq 1, y_{1} \ldots y_{l} \geq y\right\}$.

Dado $\epsilon>0$ existe $x_{1} \ldots x_{k}$ tal que $x_{1} \ldots x_{k} \geq x$ e $f\left(x_{1}\right) f\left(x_{2}\right) \ldots f\left(x_{k}\right)<F(x)+\epsilon$.

Do mesmo modo, dado $\epsilon>0$ existe $y_{1} \ldots y_{l}$ tal que $y_{1} \ldots y_{l} \geq y$ e $f\left(y_{1}\right) f\left(y_{2}\right) \ldots f\left(y_{l}\right)<$ $F(y)+\epsilon$.

Por (1) e (2) temos $x_{1} \ldots x_{k} y_{1} \ldots y_{l} \geq x y, x_{i} \geq 1, y_{i} \geq 1, k, l \in \mathbb{N}$, logo $F(x y) \leq f\left(x_{1}\right) f\left(x_{2}\right) \ldots f\left(x_{k}\right) f\left(y_{1}\right) f\left(y_{2}\right) \ldots f\left(y_{l}\right)<(F(x)+\epsilon)(F(y)+\epsilon)$, ou seja, $F(x y)<F(x) F(y)+\epsilon(F(x)+F(y)+\epsilon)$. 
Fazendo $\epsilon \rightarrow 0$ temos o desejado, isto é $F(x y) \leq F(x) F(y)$.

4) Se $g:[1,+\infty) \rightarrow[1,+\infty)$ é não decrescente submultiplicativa e dominada por $f$ então $g$ é também dominada por $F$, isto é, $F$ é a maior função submultiplicativa não decrescente menor do que $f$.

De fato, sejam $x \geq 1$ e $x_{1} \ldots x_{k} \geq x$ então $g(x) \leq g\left(x_{1} \ldots x_{k}\right) \leq g\left(x_{1}\right) \ldots g\left(x_{k}\right) \leq f\left(x_{1}\right) \ldots f\left(x_{k}\right)$.

Logo $g(x) \leq F(x)$, pois $g(x)$ é menor do que qualquer elemento de $\left\{f\left(x_{1}\right) f\left(x_{2}\right) . . f\left(x_{k}\right)\right.$ : $\left.k \in \mathbb{N}, x_{i} \geq 1, x_{1} \ldots x_{k} \geq x\right\}$. Consequentemente $g(x)$ é menor do que o ínfimo deste conjunto.

Observação 2.6 Se A é um convexo de $\mathbb{R}^{2}$ tal que $\{y:(x, y) \in A\}$ é limitado inferiormente para todo $x$ na projeção de $A$ no eixo $0 x$, segue da Observação 0.33 que a função $f$ definida por $f(x)=\inf \{y:(x, y) \in A\}$ é convexa.

De fato, sejam $x_{1}, x_{2}$ na projeção de A no eixo $0 x, 0 \leq \lambda \leq 1$ e $y_{1}, y_{2} \in \mathbb{R}$ com $f\left(x_{1}\right)<y_{1}$ e $f\left(x_{2}\right)<y_{2}$. Então existem $y_{1}^{\prime}$ e $y_{2}^{\prime}$ tais que $\left(x_{1}, y_{1}^{\prime}\right) \in A$ e $\left(x_{2}, y_{2}^{\prime}\right) \in A$.

Portanto $f\left(\lambda x_{1}+(1-\lambda) x_{2}\right)=\inf \left\{y:\left(\lambda x_{1}+(1-\lambda) x_{2}, y\right) \in A\right\} \leq \lambda y_{1}^{\prime}+(1-\lambda) y_{2}^{\prime}<$ $\lambda y_{1}+(1-\lambda) y_{2}$.

Observação 2.7 Sejam $f:[1,+\infty) \rightarrow[1,+\infty)$ e $A_{f}$ o epigráfico de $f$. Pela Observação anterior a função $F$ definida por $F(x)=$ in $f\left\{y:(x, y) \in\right.$ convexificado de $\left.A_{f}\right\}$ é convexa e é chamada de envoltória convexa de $f$.

Segue imediatamente da definição acima que $F(x)=\left\{\lambda_{1} f\left(x_{1}\right)+\lambda_{2} f\left(x_{2}\right)+\ldots+\lambda_{n} f\left(x_{n}\right)\right.$ : $\left.\sum_{i=1}^{n} \lambda_{i}=1, x=\sum_{i=1}^{n} \lambda_{i} x_{i}\right\}$

Sejam $g:[1,+\infty) \rightarrow[1,+\infty)$ e $F$ a envoltória convexa de $g$. A envoltória concava de $g$ é a função concava definida por $G(x)=-F(x)$, ou seja $G(x)=\sup \left\{\lambda_{1} g\left(x_{1}\right)+\lambda_{2} g\left(x_{2}\right)+\right.$ $\left.\ldots+\lambda_{n} g\left(x_{n}\right): \sum_{i=1}^{n} \lambda_{i}=1, x=\sum_{i=1}^{n} \lambda_{i} x_{i}\right\}$.

A envoltória concava de $g$ é a menor função concava $G:[1,+\infty) \rightarrow[1,+\infty)$ dominando $g$. De fato,

1) Demonstraremos que a envoltória concava de $g$ domina $g$.

Como $x=1 x$, temos que $g(x) \leq G(x)$. 
2) Demonstraremos agora que a envoltória concava de $g$ é a menor função concava dominando $g$, ou seja. se $f$ é concava e $g(x) \leq f(x), \forall x \geq 1$ então $G(x) \leq f(x) \forall x \geq 1$.

Seja $x=\lambda_{1} x_{1}+\lambda_{2} x_{2}+\ldots+\lambda_{n} x_{n}, \sum_{i=1}^{n} \lambda_{i}=1$,

$\lambda_{1} g\left(x_{1}\right)+\lambda_{2} g\left(x_{2}\right)+\ldots+\lambda_{n} g\left(x_{n}\right) \leq \lambda_{1} f\left(x_{1}\right)+\lambda_{2} f\left(x_{2}\right)+\ldots+\lambda_{n} f\left(x_{n}\right) \leq f(x)$.

Portanto $G(x) \leq f(x)$, pois qualquer elemento de $\left\{\lambda_{1} g\left(x_{1}\right)+\lambda_{2} g\left(x_{2}\right)+\ldots+\lambda_{n} g\left(x_{n}\right)\right.$ : $\left.\sum_{i=1}^{n} \lambda_{i}=1, x=\sum_{i=1}^{n} \lambda_{i} x_{i}\right\}$ é menor do que $f(x)$, consequentemente o supremo deste conjunto também é menor do que $f(x)$.

Definição 2.8 Dizemos que uma função $f:[1,+\infty) \rightarrow[1,+\infty)$ é supermultiplicativa se $f(x y) \geq f(x) f(y) . \forall x, y \geq 1$.

Lema 2.9 Se $g:[1,+\infty) \rightarrow[1,+\infty)$ é uma função supermultiplicativa, então sua envoltória concava também é supermultiplicativa.

\section{Demonstração:}

Sejam $\epsilon>0 . x_{1}, x_{2} \geq 1$ e $G$ a envoltória concava de $g$. Primeiro mostraremos que $\left(G\left(x_{1}\right)-\epsilon\right) \times\left(G\left(x_{2}\right)-\epsilon\right) \leq G\left(x_{1} x_{2}\right)$. Existe $\lambda_{i}, \mu_{i}, y_{i}$ e $z_{i} \in \mathbb{R}, i=1, \ldots, n$ tais que $\sum_{i=1}^{n} \lambda_{i}=1, \sum_{i=1}^{n} \mu_{i}=1, x_{1}=\lambda_{1} y_{1}+\lambda_{2} y_{2}+\ldots+\lambda_{n} y_{n}, x_{2}=\mu_{1} z_{1}+\mu_{2} z_{2}+\ldots+\mu_{n} z_{n}$, $\lambda_{1} g\left(y_{1}\right)+\lambda_{2} g\left(y_{2}\right)+\ldots+\lambda_{n} g\left(y_{n}\right) \geq G\left(x_{1}\right)-\epsilon$ e $\mu_{1} g\left(z_{1}\right)+\mu_{2} g\left(z_{2}\right)+\ldots+\mu_{n} g\left(z_{n}\right) \geq G\left(x_{2}\right)-\epsilon$.

Então,

$\left(G\left(x_{1}\right)-\epsilon\right)\left(G\left(x_{2}\right)-\epsilon\right) \leq \lambda_{1} \mu_{1} g\left(y_{1}\right) g\left(z_{1}\right)+\ldots+\lambda_{1} \mu_{n} g\left(y_{1}\right) g\left(z_{n}\right)+\lambda_{2} \mu_{1} g\left(y_{2}\right) g\left(z_{1}\right)+\ldots+$ $\lambda_{2} \mu_{n} g\left(y_{2}\right) g\left(z_{n}\right)+\ldots .+\lambda_{n} \mu_{1} g\left(y_{n}\right) g\left(z_{1}\right)+\ldots+\lambda_{n} \mu_{n} g\left(y_{n}\right) g\left(z_{n}\right) \leq \lambda_{1} \mu_{1} g\left(y_{1} z_{1}\right)+\ldots+\lambda_{1} \mu_{n} g\left(y_{1} z_{n}\right)+$ $\lambda_{2} \mu_{1} g\left(y_{2} z_{1}\right)+\ldots+\lambda_{2} \mu_{n} g\left(y_{2} z_{n}\right)+\ldots .+\lambda_{n} \mu_{1} g\left(y_{n} z_{1}\right)+\ldots+\lambda_{n} \mu_{n} g\left(y_{n} z_{n}\right)=\lambda_{1}\left(\mu_{1} g\left(y_{1} z_{1}\right)+\right.$ $\left.\ldots+\mu_{n} g\left(y_{1} z_{n}\right)\right)+\lambda_{2}\left(\mu_{1} g\left(y_{2} z_{1}\right)+\ldots+\mu_{n} g\left(y_{2} z_{n}\right)\right)+\ldots .+\lambda_{n}\left(\mu_{1} g\left(y_{n} z_{1}\right)+\ldots+\mu_{n} g\left(y_{n} z_{n}\right)\right) \leq$ $\lambda_{1} G\left(y_{1} x_{2}\right)+\lambda_{2} G\left(y_{2} x_{2}\right)+\ldots .+\lambda_{n} G\left(y_{n} x_{2}\right) \leq G\left(x_{1} x_{2}\right)$, pois $\mu_{1} y_{n} z_{1}+\mu_{2} y_{n} z_{2}+\ldots+\mu_{n} y_{n} z_{n}=y_{n}\left(\mu_{1} z_{1}+\mu_{2} z_{2}+\ldots .+\mu_{n} z_{n}\right)=y_{n} x_{2}, \lambda_{1} y_{1} x_{2}+\lambda_{2} y_{2} x_{2}+$ $\ldots .+\lambda_{n} y_{n} x_{2}=x_{2}\left(\lambda_{1} y_{1}+\lambda_{2} y_{2}+\ldots .+\lambda_{n} y_{n}\right)=x_{1} x_{2}$ e $G$ é concava.

Portanto $\left(G\left(x_{1}\right)-\epsilon\right)\left(G\left(x_{2}\right)-\epsilon\right) \leq G\left(x_{1} x_{2}\right)$. Fazendo $\epsilon \rightarrow 0$ temos $G\left(x_{1}\right) G\left(x_{2}\right) \leq$ $G\left(x_{1} x_{2}\right)$. 
Passaremos agora a construir algumas funções e estudar suas propriedades utilizando as definições anteriores.

Estas funções que construiremos serão utilizadas em alguns Lemas dos capítulos posteriores, por exemplo, $\phi$ será usada nos Lemas 4.12 e 4.13 e $h$ e $H$ serão usadas no Lema 4.12 .

Observação 2.10 Sejam $K_{0}$ um subconjunto dos naturais e $f(x)=\log _{2}(x+1)$. Definimos

a) $\phi:[1,+\infty) \rightarrow[1,+\infty)$ por

$$
\phi(x)= \begin{cases}\left(\log _{2}(x+1)\right)^{1 / 2}, & x \in K_{0} \\ \log _{2}(x+1), & x \notin K_{0}\end{cases}
$$

b) Seja $h$ a envoltória submultiplicativa de $\phi$, logo $h \leq \phi$.

c) Seja $H(x)=\frac{x}{h(x)}$ (podemos dividir por $h$, porque $h$ é o ínfimo de um conjunto cujos elementos são maiores ou iguais a 1 ).

Como h é submultiplicativa temos que $H$ é supermultiplicativa.

De fato, se $x_{1}, x_{2} \geq 1$ temos que $h\left(x_{1}\right) h\left(x_{2}\right) \geq h\left(x_{1} x_{2}\right)$ e portanto $H\left(x_{1} x_{2}\right)=$ $\frac{x_{1} x_{2}}{h\left(x_{1} x_{2}\right)} \geq \frac{x_{1}}{h\left(x_{1}\right)} \frac{x_{2}}{h\left(x_{2}\right)}=H\left(x_{1}\right) H\left(x_{2}\right)$.

d) Seja $G$ a envoltória concava de $H$, logo pela definição de envoltória concava temos $H \leq G \quad(I I)$.

Pelo Lema 2.9 G também é supermultiplicativa.

e) Seja $g(x)=\frac{x}{G(x)}$, então $g$ é submultiplicativa. De fato, como $G$ é supermultiplicativa temos que $g(x y)=\frac{x y}{G(x y)} \leq \frac{x y}{G(x) G(y)}=\frac{x}{G(x)} \frac{y}{G(y)}=g(x) g(y)$.

Lema 2.11 Sejam $g$ e $\phi$ as funções definidas acima então $\left(\log _{2}(x+1)\right)^{1 / 2} \leq g(x) \leq$ $\phi(x) \leq \log _{2}(x+1)$.

\section{Demonstração}

1) $\left(\log _{2}(x+1)\right)^{1 / 2} \leq g(x)$. 
De fato, pela definição de $\phi$ temos que $\left(\log _{2}(x+1)\right)^{1 / 2} \leq \phi(x)$. Como $h$ é a maior função submultiplicativa que é dominada por $\phi$ temos $\left(\log _{2}(x+1)\right)^{1 / 2} \leq h(x)$.

$\operatorname{Assim} \frac{x}{h(x)} \leq \frac{x}{\left(\log _{2}(x+1)\right)^{1 / 2}}, \operatorname{logo} H(x) \leq \frac{x}{\left(\log _{2}(x+1)\right)^{1 / 2}}$.

Como $G$ é a envoltória concava de $H$ e $\frac{x}{\left(\log _{2}(x+1)\right)^{1 / 2}}$ é concava, segue que $G(x) \leq$ $\frac{x}{\left(\log _{2}(x+1)\right)^{1 / 2}}, \log \frac{x}{G(x)} \geq\left(\log _{2}(x+1)\right)^{1 / 2}$. Portanto $\left(\log _{2}(x+1)\right)^{1 / 2} \leq g(x)$.

2) $g(x) \leq \phi(x)$.

De fato, $g(x)=\frac{x}{G(x)} \leq \frac{x}{H(x)}$ por (II) e $\frac{x}{H(x)}=h(x) \leq \phi(x)$ por (I).

3) $\phi(x) \leq \log _{2}(x+1)$.

Segue da definição de $\phi$.

Observação 2.12 A função $g \in \mathcal{F}$.

De fato,

1) Pelo Lema anterior temos que $\left(\log _{2}(x+1)\right)^{1 / 2} \leq g(x) \leq \log _{2}(x+1)$. Então $\left(\log _{2}(1+1)\right)^{1 / 2} \leq g(1) \leq \log _{2}(1+1)$. ou seja, $1 \leq g(x) \leq 1$. Portanto $g(1)=1$. Como $g(x) \leq \log _{2}(x+1)<x, \forall x>1$ temos que $g(x)<x, \forall x>1$.

2) $g(x) \geq\left(\log _{2}(x+1)\right)^{1 / 2}$ e como $\left(\log _{2}(x+1)\right)^{1 / 2} \stackrel{x \rightarrow+\infty}{\rightarrow}+\infty$ temos que $g(x) \stackrel{x \rightarrow+\infty}{\rightarrow}+\infty$.

Seja $x_{1}<x_{2}$ queremos provar que $g\left(x_{1}\right)<g\left(x_{2}\right)$, ou seja, $g\left(x_{1}\right)-g\left(x_{2}\right)<0$. Sabemos que $f\left(x_{1}\right)<f\left(x_{2}\right), g\left(x_{1}\right) \leq f\left(x_{1}\right)$ e $g\left(x_{2}\right) \leq f\left(x_{2}\right)$ logo $g\left(x_{1}\right)-g\left(x_{2}\right) \leq f\left(x_{1}\right)-f\left(x_{2}\right)<0$.

Portanto $g\left(x_{1}\right)<g\left(x_{2}\right)$ para $x_{1}<x_{2}$, logo $g(x)$ é estritamente crescente.

3) $\frac{(f(x))^{1 / 2}}{x^{q}} \leq \frac{g(x)}{x^{q}} \leq \frac{f(x)}{x^{q}} \operatorname{logo} \lim _{x \rightarrow+\infty} \frac{(f(x))^{1 / 2}}{x^{q}} \leq \lim _{x \rightarrow+\infty} \frac{g(x)}{x^{q}} \leq \lim _{x \rightarrow+\infty} \frac{f(x)}{x^{q}}$. Como $\lim _{x \rightarrow+\infty} \frac{(f(x))^{1 / 2}}{x^{q}}=0, \forall q>0$ e $\lim _{x \rightarrow+\infty} \frac{f(x)}{x^{q}}=0, \forall q>0$ segue que $0 \leq \lim _{x \rightarrow+\infty} \frac{g(x)}{x^{q}} \leq 0, \forall q>0$, ou seja $\lim _{x \rightarrow+\infty} \frac{g(x)}{x^{q}}=0, \forall q>0$.

4) $\frac{x}{g(x)}=\frac{x}{\frac{x}{G(x)}}=x \frac{G(x)}{x}=G(x)$ que é concava.

5) $g$ é submultiplicativa.

Por estas propriedades temos que $\frac{x}{g(x)}$ é estritamente crescente. 
Observação 2.13 Sabemos que $G$ está definida no intervalo $[1,+\infty)$. Vamos agora extender $G$ para $\mathbb{R}_{+}$, colocando $G(x)=x$ para $0 \leq x \leq 1$. Seja $\bar{G}$ essa extensão, então vamos demonstrar que $\bar{G}$ é concava, supermultiplicativa e crescente.

1) $\bar{G}$ é supermultiplicativa.

De fato, se $x_{1}$ e $x_{2}$ estão no intervalo $[0,1]$ então $\bar{G}\left(x_{1} x_{2}\right)=x_{1} x_{2}=\bar{G}\left(x_{1}\right) \bar{G}\left(x_{2}\right)$.

Se $x_{1}$ e $x_{2}$ estão no intervalo $[1,+\infty)$ então, $\bar{G}\left(x_{1} x_{2}\right)=G\left(x_{1} x_{2}\right) \geq G\left(x_{1}\right) G\left(x_{2}\right)=$ $\bar{G}\left(x_{1}\right) \bar{G}\left(x_{2}\right)$.

Se $0<x_{1} \leq 1 \leq x_{2}$, vamos estudar dois casos.

caso 1: $x_{1} x_{2} \leq 1$

$\bar{G}\left(x_{1} x_{2}\right)=x_{1} x_{2}, \bar{G}\left(x_{1}\right)=x_{1}, \bar{G}\left(x_{2}\right)=G\left(x_{2}\right)$.

$x_{1} x_{2} \geq x_{1} G\left(x_{2}\right) \Longleftrightarrow x_{2} \geq G\left(x_{2}\right)$ o que é verdade, pois $g \in \mathcal{F}, g(x)=\frac{x}{G(x)} \geq 1 \operatorname{logo}$ $G(x) \leq x$. Portanto $\bar{G}\left(x_{1} x_{2}\right) \geq \bar{G}\left(x_{1}\right) \bar{G}\left(x_{2}\right)$.

caso 2: $x_{1} x_{2}>1$

$\bar{G}\left(x_{1} x_{2}\right)=G\left(x_{1} x_{2}\right)=\frac{x_{1} x_{2}}{g\left(x_{1} x_{2}\right)}, \bar{G}\left(x_{1}\right)=x_{1}$ e $\bar{G}\left(x_{2}\right)=G\left(x_{2}\right)=\frac{x_{2}}{g\left(x_{2}\right)}$.

Temos que mostrar que $\frac{x_{1} x_{2}}{g\left(x_{1} x_{2}\right)} \geq \frac{x_{1} x_{2}}{g\left(x_{2}\right)}$, isto é, $g\left(x_{2}\right) \geq g\left(x_{1} x_{2}\right)$. Mas $x_{1} x_{2} \leq x_{2} \mathrm{e}$ como $g$ é crescente temos $g\left(x_{1} x_{2}\right) \leq g\left(x_{2}\right)$. Portanto $\bar{G}\left(x_{1} x_{2}\right) \geq \bar{G}\left(x_{1}\right) \bar{G}\left(x_{2}\right)$.

Assim temos que $\bar{G}$ é supermultiplicativa.

2) $\bar{G}$ é concava.

De fato, se $x_{1}$ e $x_{2}$ estão no intervalo $[1,+\infty)$ então temos, $\bar{G}\left(\lambda x_{1}+(1-\lambda) x_{2}\right)=G\left(\lambda x_{1}+(1-\lambda) x_{2}\right) \geq \lambda G\left(x_{1}\right)+(1-\lambda) G\left(x_{2}\right)=\lambda \bar{G}\left(x_{1}\right)+(1-\lambda) \bar{G}\left(x_{2}\right)$.

Se $x_{1}$ e $x_{2}$ estão no intervalo $[0,1]$ então, $\bar{G}\left(\lambda x_{1}+(1-\lambda) x_{2}\right)=\lambda x_{1}+(1-\lambda) x_{2}=\lambda \bar{G}\left(x_{1}\right)+(1-\lambda) \bar{G}\left(x_{2}\right)$.

Se $0<x_{1} \leq 1<x_{2}$, quero mostrar que $\bar{G}\left(\lambda x_{1}+(1-\lambda) x_{2}\right) \geq \lambda \bar{G}\left(x_{1}\right)+(1-\lambda) \bar{G}\left(x_{2}\right)$. caso 1: $\lambda x_{1}+(1-\lambda) x_{2} \leq 1$ $\bar{G}\left(\lambda x_{1}+(1-\lambda) x_{2}\right)=\lambda x_{1}+(1-\lambda) x_{2}, \bar{G}\left(x_{1}\right)=x_{1}, \bar{G}\left(x_{2}\right)=G\left(x_{2}\right)$. $\lambda x_{1}+(1-\lambda) x_{2} \geq \lambda x_{1}+(1-\lambda) G\left(x_{2}\right) \Longleftrightarrow(1-\lambda) x_{2} \geq(1-\lambda) G\left(x_{2}\right) \Longleftrightarrow x_{2} \geq G\left(x_{2}\right), o$ que é verdade pois $g(x)=\frac{x}{G(x)} \geq 1 \operatorname{logo} G(x) \leq x$.

Portanto $\bar{G}\left(\lambda x_{1}+(1-\lambda) x_{2}\right) \geq \lambda \bar{G}\left(x_{1}\right)+(1-\lambda) \bar{G}\left(x_{2}\right)$. caso 2: $\lambda x_{1}+(1-\lambda) x_{2}>1$ 
$\bar{G}\left(\lambda x_{1}+(1-\lambda) x_{2}\right)=G\left(\lambda x_{1}+(1-\lambda) x_{2}\right)=\frac{\lambda x_{1}+(1-\lambda) x_{2}}{g\left(\lambda x_{1}+(1-\lambda) x_{2}\right)}, \bar{G}\left(x_{1}\right)=x_{1}, \bar{G}\left(x_{2}\right)=G\left(x_{2}\right)$.

Temos que mostrar que $\bar{G}\left(\lambda x_{1}+(1-\lambda) x_{2}\right) \geq \lambda \bar{G}\left(x_{1}\right)+(1-\lambda) \bar{G}\left(x_{2}\right)$.

Sejam $x_{3}=\lambda x_{1}+(1-\lambda) x_{2}, y_{1}=r(x)$ e $y_{2}=s(x)$ as retas passando respectivamente pelos pontos $\left(x_{1}, x_{1}\right),\left(x_{2}, G\left(x_{2}\right)\right)$ e $(1,1),\left(x_{2}, G\left(x_{2}\right)\right)$. Como $G$ é concava, temos que $s\left(x_{3}\right)<G\left(x_{3}\right)$ e como $x_{1}<1$, temos $r\left(x_{3}\right)<s\left(x_{3}\right)$ logo $r\left(x_{3}\right)<G\left(x_{3}\right)$. Ou seja, $\lambda x_{1}+(1-\lambda) G\left(x_{2}\right)<G\left(\lambda x_{1}+(1-\lambda) x_{2}\right), \operatorname{assim} \lambda \bar{G}\left(x_{1}\right)+(1-\lambda) \bar{G}\left(x_{2}\right)<\bar{G}\left(\lambda x_{1}+(1-\lambda) x_{2}\right)$.

Portanto $\bar{G}\left(\lambda x_{1}+(1-\lambda) x_{2}\right) \geq \lambda \bar{G}\left(x_{1}\right)+(1-\lambda) \bar{G}\left(x_{2}\right)$. Assim temos que $\bar{G}$ é concava.

3) $\bar{G}$ é crescente.

De fato, se $x_{1}$ e $x_{2}$ estão em $[0,1]$ então sejam $x_{1}<x_{2} \operatorname{logo} G\left(x_{1}\right)<G\left(x_{2}\right)$, pois $G\left(x_{1}\right)=x_{1}<x_{2}=G\left(x_{2}\right)$.

Se $x_{1}$ e $x_{2}$ estão em $[1,+\infty)$ então temos que $\bar{G}=G$ e $G$ é crescente.

Se $0 \leq x_{1} \leq 1 \leq x_{2}, \bar{G}\left(x_{1}\right)=x_{1}, \bar{G}\left(x_{2}\right)=G\left(x_{2}\right)=\frac{x_{2}}{g\left(x_{2}\right)}$.

$x_{1}<\frac{x_{2}}{g\left(x_{2}\right)} \Longleftrightarrow x_{1} g\left(x_{2}\right)<x_{2}$ mas $x_{1} g\left(x_{2}\right)<x_{1} x_{2}<x_{2}$.

Portanto temos que se $x_{1}<x_{2}$ então $\bar{G}\left(x_{1}\right)<\bar{G}\left(x_{2}\right)$, ou seja, $\bar{G}$ é crescente. 


\section{Capítulo 3}

\section{O espaço de Thomas Schlumprecht}

Inicialmente apresentaremos uma condição suficiente para que um espaço normado separável possa ser renormado de tal maneira que ele não contenha nenhuma sequência básica $C$-incondicional onde $C \in \mathbb{R}, C>1$ é pré-fixado (Veja Teorema 3.3).

Esta condição foi obtida por Gowers e Maurey, [11], e eles próprios mostraram que o espaço de Thomas Schlumprecht a satisfaz (Veja Teorema 3.22 e Corolário 3.23).

No entanto, neste capítulo, estudaremos o espaço de Thomas Schlumprecht não só por ter a propriedade acima citada, mas também por ter inspirado a construção do espaço de Gowers e Maurey que será apresentada no próximo capítulo.

A teoria apresentada neste capítulo pode ser encontrada em [11].

\subsection{Conjuntos assintóticos}

Antes de provarmos o Teorema 3.3 precisaremos de duas definições.

Seja $X$ um espaço normado e seja $S(X)$ sua esfera unitária, isto é $S(x)=\{x \in$ $X:\|x\|=1\}$.

Definição 3.1 Um subconjunto $A \subset S(X)$ é assintótico se $A \cap S(Y) \neq \emptyset$ para todo subespaço de dimensão infinita $Y$ de $\mathrm{X}$. 
Definição 3.2 Dizemos que uma sequência $\left(A_{n}\right)_{n}$ de subconjuntos de $S(X)$ e uma sequência de subconjuntos $\left(A_{n}^{*}\right)_{n}$ de $B\left(X^{*}\right)$ formam um sistema biortogonal assintótico com constante $\delta$ se as seguintes condições são satisfeitas :

a) Para todo $n \in \mathbb{N}$, o conjunto $A_{n}$ é assintótico.

b) Para todo $n \in \mathbb{N}$ e todo $x \in A_{n}$, existe $x^{*} \in A_{n}^{*}$ tal que $x^{*}(x)>1-\delta$.

c) Para todo $n, m \in \mathbb{N}, n \neq m$, todo $x \in A_{n}$, e todo $x^{*} \in A_{m}^{*}$ tem-se $\left|x^{*}(x)\right|<\delta$.

Teorema 3.3 Sejam $0<\delta<1 / 100$ e X um espaço normado separável contendo um sistema biortogonal assintótico com constante $\delta$. Então existe uma norma equivalente em $X$ tal que nenhuma sequência é $1 / \sqrt{100 \delta}$-incondicional.

\section{Demonstração:}

Seja $\|\cdot\|$ a norma original de $X$, e sejam $\left(A_{n}\right)_{n}$ e $\left(A_{n}^{*}\right)_{n}$ um sistema biortogonal assintótico com constante $\delta$. Para cada $n$ vamos construir um subconjunto enumerável $Z_{n}^{*}$ de $A_{n}^{*}$, tal que para todo $x \in A_{n}$ existe $x^{*} \in Z_{n}^{*}$ com $x^{*}(x)>1-2 \delta$, da seguinte maneira.

$X$ é separável e os $A_{n^{\prime} s}$ são subconjuntos de $X$, logo os $A_{n^{\prime} s}$ também são separáveis. Seja $\left\{d_{i}\right\}_{i}$ denso em $A_{n}$. Para cada $i$ existe $x_{i}^{*} \in A_{n}^{*}$ tal que $x_{i}^{*}\left(d_{i}\right)>1-\delta$. Consideremos $Z_{n}^{*}=\left\{x_{i}^{*}\right\}_{i}, \operatorname{logo} Z_{n}^{*} \subset A_{n}^{*}$ e é enumerável.

Temos também que $\forall x \in A_{n}, \exists x^{*} \in Z_{n}^{*}$ tal que $x^{*}(x)>1-2 \delta$, pois se $x \in A_{n}$, como $\left\{d_{i}\right\}_{i}$ é denso em $A_{n}$ existe $\left(d_{i}\right)_{j}$ tal que $\left\|x-d_{i},\right\|<\delta$.

Consideremos $x_{i}^{*} \in Z_{n}^{*}$ e calculemos o valor de $x_{i}^{*}(x)$. $\left|x_{i}^{*}(x)-x_{i}^{*}\left(d_{i_{j}}\right)\right|=\left|x_{i}^{*}\left(x-d_{i_{j}}\right)\right| \leq\left\|x_{i}^{*}\right\|\left\|x-d_{i_{j}}\right\| \leq\left\|x-d_{i},\right\|<\delta$, pois $\left\|x_{i}^{*}\right\| \leq 1$.

Portanto temos $-\delta<x_{i}^{*}(x)-x_{i}^{*}\left(d_{i,}\right)<\delta$, logo $x_{i}^{*}\left(d_{i,}\right)-\delta<x_{i}^{*}(x)<x_{i}^{*}\left(d_{i j}\right)+\delta$.

$\operatorname{Assim} x_{i}^{*}(x)>x_{i}^{*}\left(d_{i},\right)-\delta>1-\delta-\delta=1-2 \delta$, como queriamos.

Sejam $Z^{*}=\bigcup_{n=1}^{\infty} Z_{n}^{*}$ e $\sigma$ uma função injetora,

$$
\sigma: \tau \longrightarrow \mathbb{N}
$$

onde $\tau$ é a coleção de sequências finitas de $Z^{*}$ ( $\sigma$ existe porque $\tau$ é enumerável ).

Agora definiremos uma coleção de funcionais lineares continuos que chamaremos de funcionais especiais. Uma sequência especial de funcionais de comprimento $r$ é uma 
sequência da forma $z_{1}^{*}, z_{2}^{*}, \ldots, z_{r}^{*}$ onde $z_{1}^{*} \in Z_{1}^{*}$ e para $1 \leq i<r, z_{i+1}^{*} \in Z_{\sigma\left(z_{1}^{*}, \ldots, z_{i}^{*}\right)}^{*}$.

Um funcional especial de comprimento $r$ é simplesmente a soma de uma sequência especial de comprimento $r$. Denotaremos por $\Gamma_{r}$ a coleção dos funcionais especiais de comprimento $r$.

Vamos definir agora uma nova norma em $X$ da seguinte forma. Seja $r=\left\lfloor\delta^{-1 / 2}\right\rfloor$, ou seja $r$ será igual a parte inteira de $\delta^{-1 / 2}$, e definamos $\|||$.$\| por$

$$
\||x|\|=\max \left\{\|x\|, r \max \left\{\left|z^{*}(x)\right|: z^{*} \in \Gamma_{r}\right\}\right\} .
$$

As normas $\|$.$\| e \|||$.$\| são equivalentes. De fato, para cada x \in X$, temos que $\||x|\|=$ $\|x\|$ ou $\||x|\|=r \max \left\{\left|z^{*}(x)\right|: z^{*} \in \Gamma_{r}\right\}$.

Mas $r \max \left\{\left|z^{*}(x)\right|: z^{*} \in \Gamma_{r}\right\} \leq r . r\|x\|=r^{2}\|x\|$, pois $\left|z^{*}(x)\right| \leq\left\|z^{*}\right\|\|x\| \leq r\|x\|$.

Logo $\||x|\| \leq r^{2}\|x\|$, pois $r \geq 1$ ( na verdade $r \geq 10$ ) e pela definição da norma temos $\||x|\| \geq\|x\|$ portanto $\|x\| \leq\||x|\| \leq r^{2}\|x\|$.

Seja $\left(x_{i}\right)_{i}$ uma sequência qualquer de vetores linearmente independentes em $X$. Vamos mostrar que essa sequência não é $(r-2) / 5$-incondicional na norma $\| \mid$.|||

Faremos isto construindo uma sequencia de vetores $z_{1}, \ldots, z_{r}$ gerados por $\left(x_{i}\right)_{i}$ e tais que os vetores que geram $z_{1}$ não geram os demais $z_{i^{\prime}}$ e assim por diante, com a propriedade de que $(r-2)\left\||| \sum_{i=1}^{r}(-1)^{i} z_{i}|\|<5\||\left|\sum_{i=1}^{r} z_{i}\right|\right\|$.

Com isto teremos provado o teorema, pois $\left(x_{i}\right)_{i}$ não será $(r-2) / 5$-incondicional uma vez que $\left\|\left|\sum \varepsilon_{n} a_{n} x_{n}\right|\right\| \geq(r-2) / 5\left\|\left|\sum a_{n} x_{n}\right|\right\| \mid, \forall\left(a_{n}\right)_{n}$ e $\forall\left(\varepsilon_{n}\right)_{n}$ de módulo no máximo um, e como veremos abaixo $(r-2) / 5>1 / \sqrt{100 \delta} \operatorname{logo}\left(x_{i}\right)_{i}$ não será $1 / \sqrt{100 \delta}$-incondicional.

Primeiro vamos verificar que $(r-2) / 5>1 / \sqrt{100 \delta}$.

Sabemos que $\frac{1}{\sqrt{\delta}}<r+1, \log \frac{1}{10 \sqrt{\delta}}<\frac{r+1}{10}$. Então basta demonstrarmos que $\frac{r+1}{10}<\frac{r-2}{5}$ e teremos o desejado.

$\frac{r+1}{10}<\frac{r-2}{5} \Longleftrightarrow 5 r+5<10 r-20 \Longleftrightarrow r>5$, o que é verdadeiro.

Passaremos, agora, a construção dos $z_{i^{\prime} s}$. Seja $X_{1}$ o subespaço algébrico gerado por $\left(x_{i}\right)_{i}$ em $X$. Como $A_{1}$ é assintótico, podemos achar $z_{1} \in A_{1} \cap X_{1}$. Isto implica que $z_{1}$ tem norma 1 e é combinação linear de um número finito de $x_{i^{\prime} s}$. 
Em seguida escolhamos, como provamos acima, $z_{1}^{*} \in Z_{1}^{*}$ tal que $z_{1}^{*}\left(z_{1}\right)>1-2 \delta$. Agora seja $X_{2}$ o subespaço algébrico gerado por todos os $x_{i^{\prime} s}$ não usados na combinação linear de $z_{1}$. Como $A_{\sigma\left(z_{1}^{*}\right)}$ é assintótico podemos achar $z_{2} \in A_{\sigma\left(z_{1}^{*}\right)} \cap X_{2}$ de norma 1 . Novamente escolhamos $z_{2}^{*} \in Z_{\sigma\left(z_{1}^{*}\right)}^{*}$ tal que $z_{2}^{*}\left(z_{2}\right)>1-2 \delta$. Continuando este processo, nós obtemos as sequências $z_{1}, \ldots, z_{r}$ e $z_{1}^{*}, \ldots, z_{r}^{*}$ com as seguintes propriedades:

1) $\left\|z_{i}\right\|=1$ para cada $\mathrm{i}$.

2) $z_{1}^{*} \in Z_{1}^{*}$ e $z_{i+1}^{*} \in Z_{\sigma\left(z_{1}^{*}, \ldots, z_{i}^{*}\right)}^{*}$ para $1 \leq i<r$, isto é, $z_{1}^{*}, \ldots, z_{r}^{*}$ é uma sequência especial de funcionais de comprimento $r$.

3) $z_{i}^{*}\left(z_{i}\right)>1-2 \delta$ para cada i.

4) Como $\sigma$ é injetora, os $z_{i^{\prime} s}^{*}$ pertencem a diferentes $A_{n^{\prime} s}^{*}$ e portanto $\left|z_{i}^{*}\left(z_{j}\right)\right|<\delta<2 \delta$, quando $i \neq j$.

Estimaremos agora em a) o valor de $\left\|\left|\sum_{i=1}^{r} z_{i}\right|\right\|$ e depois em b) o valor de $\left\|\left|\sum_{i=1}^{r}(-1)^{i} z_{i}\right|\right\|$.

a) $\left\|\left|\sum_{i=1}^{r} z_{i}\right|\right\| \geq \max \left\{\left|z^{*}\left(\sum_{i=1}^{r} z_{i}\right)\right|: z^{*} \in \Gamma_{r}\right\} \geq r\left|z^{*}\left(\sum_{i=1}^{r} z_{i}\right)\right| \geq r z^{*}\left(\sum_{i=1}^{r} z_{i}\right)$.

Como $z_{1}^{*}, \ldots, z_{r}^{*}$ é uma sequência especial de funcionais de comprimento $r$, podemos tomar $z^{*}=\sum_{i=1}^{r} z_{i}^{*} \operatorname{logo}$

$\left\|\left|\sum_{i=1}^{r} z_{i}\right|\right\| \geq r\left(\sum_{i=1}^{r} z_{i}^{*}\right)\left(\sum_{i=1}^{r} z_{i}\right)=r\left(\sum_{i=1}^{r} z_{i}^{*}\left(\sum_{i=1}^{r} z_{i}\right)\right)>r(r(1-2 \delta)-2 \delta r(r-1))=r\left(r-2 \delta r^{2}\right) \geq$ $r(r-2)$, pois $r-2 \delta r^{2} \geq r-2 \Longleftrightarrow \delta r^{2} \leq 1 \Longleftrightarrow r^{2} \leq 1 / \delta \Longleftrightarrow r \leq 1 / \sqrt{\delta}$, o que é verdadeiro.

b) Seja $\left(w_{i}^{*}\right)_{i=1}^{r}$ uma sequência especial de comprimento $r$ qualquer e seja $t$ o maior dos índices $\mathrm{i}$ tal que $w_{i}^{*}=z_{i}^{*}$ ou $t$ igual a zero se $w_{1}^{*} \neq z_{1}^{*}$. Então, $\left|\sum_{i=1}^{r}(-1)^{i} w_{i}^{*}\left(z_{i}\right)\right| \leq\left|\sum_{i=1}^{t}(-1)^{i} w_{i}^{*}\left(z_{i}\right)\right|+\left|w_{t+1}^{*}\left(z_{t+1}\right)\right|+\sum_{i=t+2}^{r}\left|w_{i}^{*}\left(z_{i}\right)\right|$.

A seguir obteremos uma majoração para cada um dos três somandos do segundo membro da desigualdade.

I) Como $\sigma$ é injetora, $w_{i}^{*}$ e $z_{j}^{*}$ são escolhidos de diferentes $A_{n}^{*}$ quando $i \neq j$ ou $i=j>t+1$. Pela propriedade (c) da definição de conjuntos assintóticos temos $\left|w_{i}^{*}\left(z_{j}\right)\right|<\delta<2 \delta$. Em particular, $\sum_{i=t+2}^{r}\left|w_{i}^{*}\left(z_{i}\right)\right|<2 \delta(r-(t+2))<2 \delta r$. 
II) Quando $i \leq t$ nós sabemos que $\left.1-2 \delta<w_{i}^{*}\left(z_{i}\right) \leq 1 \quad{ }^{*}\right)$. Explicitando a somatória abaixo temos,

$\left|\sum_{i=1}^{t}(-1)^{i} w_{i}^{*}\left(z_{i}\right)\right|=\left|-w_{1}^{*}\left(z_{1}\right)+w_{2}^{*}\left(z_{2}\right)-w_{3}^{*}\left(z_{3}\right)+\ldots+(-1)^{t} w_{t}^{*}\left(z_{t}\right)\right|$ então usando $\left(^{*}\right)$ obtemos $-w_{i}^{*}\left(z_{i}\right)+w_{j}^{*}\left(z_{j}\right) \leq 2 \delta-1+1=2 \delta$.

Não sabemos se o número de somandos é par ou ímpar, por isso vamos estudar os dois casos.

1) o número de somandos, t, é par

$\left|\sum_{i=1}^{t}(-1)^{i} w_{i}^{*}\left(z_{i}\right)\right| \leq t / 2.2 \delta=\delta t<1+t \delta$.

2) o número de somandos, t, é impar

$\left|\sum_{i=1}^{t-1}(-1)^{i} w_{i}^{*}\left(z_{i}\right)\right|+\left|w_{t}^{*}\left(z_{t}\right)\right| \leq 1+(t-1) / 2.2 \delta \leq 1+t \delta$.

De 1) e 2) segue que $\left|\sum_{i=1}^{t}(-1)^{i} w_{i}^{*}\left(z_{i}\right)\right| \leq 1+\delta t$.

III) $\left|w_{t+1}^{*}\left(z_{t+1}\right)\right| \leq\left\|w_{t+1}^{*}\right\|\left\|z_{t+1}\right\| \leq 1$.

Por I), II) e III) temos $\left|\sum_{i=1}^{r}(-1)^{i} w_{i}^{*}\left(z_{i}\right)\right| \leq 1+\delta t+1+2 \delta r \leq 1+\delta r+1+2 \delta r=2+3 \delta r$.

Temos que $\sum_{i \neq j}\left|w_{i}^{*}\left(z_{j}\right)\right| \leq 2 \delta r(r-1)$, pois temos $r w_{i^{\prime} s}^{*}$ e $r z_{j^{\prime} s}$ e $\left|w_{i}^{*}\left(z_{j}\right)\right|<\delta<2 \delta$.

Pela desigualdade triangular temos $\left\|\sum_{i=1}^{r}(-1)^{i} z_{i}\right\| \leq \sum_{i=1}^{r}\left\|(-1)^{i} z_{i}\right\|=$ $\sum_{i=1}^{r}\left|(-1)^{i}\right|\left\|z_{i}\right\|=r$, pois $\left\|z_{i}\right\|=1 \quad \forall i$.

Pela definição de ||$|| \mid$. e pelas estimativas acima temos $\left\|\left|\sum_{i=1}^{r}(-1)^{i} z_{i}\right|\right\|=\left\|\sum_{i=1}^{r}(-1)^{i} z_{i}\right\| \leq r$ ou $\left\|\left|\sum_{i=1}^{r}(-1)^{i} z_{i}\right|\right\|=r \max \left\{\left|\left(\sum_{i=1}^{r} w_{i}^{*}\right)\left(\sum_{i=1}^{r}(-1)^{i} z_{i}\right)\right|:\right.$ $\left.\left(\sum_{i=1}^{r} w_{i}^{*}\right) \in \Gamma_{r}\right\} \leq r \max \left\{\left|\sum_{i=1}^{r}(-1)^{i} w_{i}^{*}\left(z_{i}\right)\right|+\sum_{i \neq j}\left|w_{i}^{*}\left(z_{j}\right)\right|:\left(\sum_{i=1}^{r} w_{i}^{*}\right) \in \Gamma_{r}\right\} \leq$ $r(2+3 \delta r+2 \delta r(r-1))$.

Nos dois casos temos que 
$\left\|\left|\sum_{i=1}^{r}(-1)^{i} z_{i}\right|\right\| \leq r(2+3 \delta r+2 \delta r(r-1))=r\left(2+3 \delta r+2 \delta r^{2}-2 \delta r\right)=r\left(2+\delta r+2 \delta r^{2}\right)$.

Como $r \leq 1 / \sqrt{\delta}$ temos que $\delta r<1 / 10<1$ e $\delta r^{2}<1$, logo $\left\|1 \sum_{i=1}^{r}(-1)^{i} z_{i}\right\|<$ $r(2+1+2)<5 r$.

Sendo assim $(r-2)\left\|\left|\sum_{i=1}^{r}(-1)^{i} z_{i}\right|\right\|<5 r(r-2)$ e como $\left\|\left|\sum_{i=1}^{r} z_{i}\right|\right\| \geq r(r-2)$ segue que $(r-2)\left\||| \sum_{i=1}^{r}(-1)^{i} z_{i}|\|<5\||\left|\sum_{i=1}^{r} z_{i}\right|\right\|$.

\subsection{O espaço de Schlumprecht}

Depois de termos apresentado no Teorema 3.3 uma condição suficiente para que um espaço de Banach separável possa ser renormado de forma a não conter nenhuma sequência básica $\mathrm{C}$-incondicional com $C \in \mathbb{R}, C>1$, pré-fixado apresentaremos um espaço de Banach que satisfaz esta condição.

Esse espaço foi construido em 1991 por Schlumprecht, [22], e é uma construção " tipo Tsirelson" por ser definido indutivamente de maneira análoga ao espaço $T$ de Tsirelson, capítulo 1 , como veremos a seguir.

Consideremos $\mathcal{F}$ a classe de funções definida no capítulo anterior e $f(x)=\log _{2}(x+1)$.

Em $c_{00}$ definimos por indução em $k \in \mathbb{N}$ a norma $|\cdot|_{k}$.

Para $x \in c_{00}$, seja $|x|_{0}=\max _{n \in \mathbb{N}}\left|x_{n}\right|$. Assumindo que $|x|_{k}$ está definida para $k \in \mathbb{N}$ colocamos,

$$
|x|_{k+1}=\max _{l \in \mathbb{N}} \frac{1}{f(l)} \sum_{i=1}^{l}\left|E_{i}(x)\right|_{k},
$$

onde o máximo é tomado sobre todas as sequências de subconjuntos $E_{i} \subset \mathbb{N}$ tais que $E_{1}<E_{2}<\ldots<E_{l}$.

Claramente $|x|_{0}$ é norma em $c_{00}$.

Agora assumiremos que $|.|_{k}$ é norma em $c_{00}$ e provaremos que $|\cdot|_{k+1}$ também é norma em $c_{00}$. De fato. sejam $x$ e $y$ elementos de $c_{00}$. 


$$
\begin{aligned}
& \text { 1) }|x|_{k+1} \geq 0 \text {. pois }|x|_{k+1}=\max _{l \in \mathbb{N}} \frac{1}{f(l)} \sum_{i=1}^{l}\left|E_{i}(x)\right|_{k} \geq 0 . \\
& |x|_{k+1}=0 \Longleftrightarrow \max _{l \in \mathbb{N}} \frac{1}{f(l)} \sum_{i=1}^{l}\left|E_{i}(x)\right|_{k}=0 \Longleftrightarrow\left|E_{i}(x)\right|_{k}=0 \quad \forall i \Longleftrightarrow x=0 . \\
& \text { 2) }|\lambda x|_{k+1}=\max _{l \in \mathbb{N}} \frac{1}{f(l)} \sum_{i=1}^{l}\left|E_{i}(\lambda x)\right|_{k}=\max _{l \in \mathbb{N}} \frac{1}{f(l)} \sum_{i=1}^{l}\left|\lambda E_{i}(x)\right|_{k}=\max _{l \in \mathbb{N}} \frac{1}{f(l)} \sum_{i=1}^{l}|\lambda|\left|E_{i}(x)\right|_{k}= \\
& |\lambda|_{l \in \mathbb{N}} \frac{1}{f(l)} \sum_{i=1}^{l}\left|E_{i}(x)\right|_{k}=|\lambda||x|_{k+1}, \forall \lambda \in \mathbb{R} . \\
& 3)|x+y|_{k+1}=\max _{l \in \mathbb{N}} \frac{1}{f(l)} \sum_{i=1}^{l}\left|E_{i}(x+y)\right|_{k}=\max _{l \in \mathbb{N}} \frac{1}{f(l)} \sum_{i=1}^{l}\left|E_{i}(x)+E_{i}(y)\right|_{k} \leq \\
& \max _{l \in \mathbb{N}} \frac{1}{f(l)} \sum_{i=1}^{l}\left(\left|E_{i}(x)\right|_{k}+\left|E_{i}(y)\right|_{k}\right) \leq \max _{l \in \mathbb{N}} \frac{1}{f(l)} \sum_{i=1}^{l}\left|E_{i}(x)\right|_{k}+\max _{l \in \mathbb{N}} \frac{1}{f(l)} \sum_{i=1}^{l}\left|E_{i}(y)\right|_{k}=|x|_{k+1}+ \\
& |y|_{k+1} .
\end{aligned}
$$

Por 1), 2) e 3) temos que $|x|_{k+1}$ é norma em $c_{00}$ para todo $k \in \mathbb{N}$.

Estudaremos agora algumas propriedades de $|\cdot|_{k}$.

1) $\left(|x|_{k}\right)_{k}$ é crescente para qualquer $x \in c_{00}$.

$$
\begin{aligned}
\text { De fato, }|x|_{k+1} & =\max _{l \in \mathbb{N}} \frac{1}{f(l)} \sum_{i=1}^{l}\left|E_{i}(x)\right|_{k}, \text { fazendo } l=1 \text { e } E_{1}=\operatorname{ran}(x) \text { temos que } \\
|x|_{k+1} \geq \frac{1}{f(1)}\left|E_{1}(x)\right|_{k} & =|x|_{k} .
\end{aligned}
$$

Portanto temos que $|x|_{k+1} \geq|x|_{k}$ para qualquer $x \in c_{00}$.

2) $|x|_{k} \leq\|x\|_{l_{1}}$ para qualquer $x \in c_{00}$ e $k \in \mathbb{N}$.

De fato, $|x|_{0}=\max _{n \in \mathbb{N}}\left|x_{n}\right| \leq \sum_{i=1}^{\infty}\left|x_{n}\right|=\|x\|_{l_{1}}$.

Suponhamos que $|x|_{k} \leq\|x\|_{l_{1}}$. Vamos provar que $|x|_{k+1} \leq\|x\|_{l_{1}}$. $|x|_{k+1}=\max _{l \in \mathbb{N}} \frac{1}{f(l)} \sum_{i=1}^{l}\left|E_{i}(x)\right|_{k} \leq \max _{l \in \mathbb{N}} \frac{1}{f(l)} \sum_{i=1}^{l}\left\|E_{i}(x)\right\|_{l_{1}} \leq \max _{l \in \mathbb{N}} \sum_{i=1}^{l}\left\|E_{i}(x)\right\|_{l_{1}} \leq\|x\|_{l_{1}}$.

Finalmente definimos, para $x \in c_{00},\|x\|=\max _{k \in \mathbb{N}}|x|_{k}$. É claro que $\|\cdot\|$ é uma norma em $c_{00}$. O completado de $c_{00}$ com essa norma é o espaço de Thomas Schlumprecht que 
denotaremos por TS.

Apresentaremos agora alguns resultados relativos a norma do espaço ( Observação 3.4 e Proposição 3.5) que serão úteis na verificação dos Lemas obtidos por Gowers e Maurey para o espaço de Thomas Schlumprecht.

Esses Lemas permitirão demonstrar o Teorema abaixo:

Teorema Seja $\delta \in \mathbb{R}, 0<\delta<1$. Então existe no espaço de Thomas Schlumprecht um sistema biortogonal assintótico com constante $\delta$.

Observação 3.4 máximo de $\left(|x|_{k}\right)_{k}$ é assumido .

De fato, por definição $|x|_{k+1}=\max _{l \in \mathbb{N}} \frac{1}{f(l)} \sum_{i=1}^{l}\left|E_{i}(x)\right|_{k}$ para qualquer $x \in c_{00}$ e $k \in \mathbb{N}$.

Então o máximo da definição de $|x|_{k+1}$ é assumido para $l=1$ e neste caso teremos $|x|_{k+1}=|x|_{k}$ ou o máximo é assumido para $l \geq 2$.

Suponha que para algum $x \in c_{00}$ o máximo de $\left(|x|_{k}\right)_{k}$ não exista, logo $|x|_{1}<|x|_{2}<$ $|x|_{3}<\ldots$ e portanto o máximo na definição de $|x|_{k+1}$ não é assumido quando $l=1$, para qualquer $k$. Afirmação, neste caso, $|x|_{k+1} \leq \frac{1}{f(2)^{k}}\|x\|_{l_{1}}$.

Faremos a demonstração por indução em $k$.

Para $k=0$, $|x|_{1}=\max _{l \in \mathbb{N}} \frac{1}{f(l)} \sum_{i=1}^{l}\left|E_{i}(x)\right|_{0} \leq \max _{l \in \mathbb{N}} \sum_{i=1}^{l}\left|E_{i}(x)\right|_{0} \leq \max _{l \in \mathbb{N}} \sum_{i=1}^{l}\left\|E_{i}(x)\right\|_{l_{1}} \leq\|x\|_{l_{1}}$.

Portanto $|x|_{1} \leq \frac{1}{f(2)^{0}}\|x\|_{l_{1}}$.

Para $k=1$,

$|x|_{2}=\max _{l \in \mathbb{N}} \frac{1}{f(l)} \sum_{i=1}^{l}\left|E_{i}(x)\right|_{1} \leq \max _{l \in \mathbb{N}} \frac{1}{f(2)} \sum_{i=1}^{l}\left|E_{i}(x)\right|_{1}=\frac{1}{f(2)} \max _{l \in \mathbb{N}} \sum_{i=1}^{l}\left|E_{i}(x)\right|_{1} \leq$ $\frac{1}{f(2)} \max _{l \in \mathbb{N}} \sum_{i=1}^{l}\left\|E_{i}(x)\right\|_{l_{1}} \leq \frac{1}{f(2)}\|x\|_{l_{1}}$. Portanto, $|x|_{2} \leq \frac{1}{f(2)}\|x\|_{l_{1}}$.

Agora suponhamos que $|x|_{k} \leq \frac{1}{f(2)^{k}}\|x\|_{l_{1}}$, vamos provar que vale para $k+1$.

$|x|_{k+1}=\max _{l \in \mathbb{N}} \frac{1}{f(l)} \sum_{i=1}^{l}\left|E_{i}(x)\right|_{k} \leq \max _{l \in \mathbb{N}} \frac{1}{f(2)} \sum_{i=1}^{l}\left|E_{i}(x)\right|_{k}=\frac{1}{f(2)} \max _{l \in \mathbb{N}} \sum_{i=1}^{l}\left|E_{i}(x)\right|_{k} \leq$ 
$\leq \frac{1}{f(2)} \max _{l \in \mathbb{N}} \sum_{i=1}^{l} \frac{1}{f(2)^{k}}\left\|E_{i}(x)\right\|_{l_{1}}=\frac{1}{f(2)^{k+1}} \max _{l \in \mathbb{N}} \sum_{i=1}^{l}\left\|E_{i}(x)\right\|_{l_{1}} \leq \frac{1}{f(2)^{k+1}}\|x\|_{l_{1}}$.

Portanto $|x|_{k} \leq \frac{1}{f(2)^{k}}\|x\|_{l_{1}}$ para qualquer $k$ e $x \in c_{00}$

Mas $\left(^{*}\right)$ implica que $|x|_{k} \stackrel{k \rightarrow+\infty}{\rightarrow} 0$, absurdo, pois $\left(|x|_{k}\right)_{k}$ é uma sequência positiva e estritamente crescente.

Proposição 3.5 Seja TS o espaço de Thomas Schlumprecht.

a) $\left(e_{i}\right)_{i}$ é base 1-incondicional para TS.

b) Para $x \in T S$ tem-se

$$
\|x\|=\max \left\{|x|_{0}, \sup _{l \geq 2} \frac{1}{f(l)} \sum_{i=1}^{l}\left\|E_{i}(x)\right\|\right\},
$$

onde o máximo é tomado sobre todas as sequências de subconjuntos $E_{i} \subset \mathbb{N}$ tais que $E_{1}<E_{2}<\ldots<E_{l}$ e $|x|_{0}=\sup _{n \in \mathbb{N}}\left|x_{n}\right|$ para $x=\sum_{i=1}^{\infty} x_{i} e_{i} \in T S$.

\section{Demonstração:}

a) Faremos a demonstração em 4 partes.

1) $\left\|e_{i}\right\|=1, \forall i$.

De fato, pela propriedade 1) da norma temos $1=\left|e_{i}\right|_{0} \leq\left|e_{i}\right|_{1} \leq \ldots$, ou seja, $1 \leq\left|e_{i}\right|_{k}$, $\forall k$ e pela propriedade 2) da norma temos $\left|e_{i}\right|_{k} \leq\left\|e_{i}\right\|_{l_{1}}=1, \forall k$. Portanto $\left|e_{i}\right|_{k}=1, \forall k$, consequentemente $\left\|e_{i}\right\|=1, \forall i$.

2) $\left(e_{i}\right)_{i}$ é sequência básica.

Pelo Teorema 0.3 é suficiente mostrarmos que

$$
\left\|\sum_{i=1}^{m} a_{i} e_{i}\right\| \leq\left\|\sum_{i=1}^{n} a_{i} e_{i}\right\|
$$

para qualquer sequência de escalares $\left(a_{n}\right)_{n}$ e inteiros $m<n$.

$\left|\sum_{i=1}^{m} a_{i} e_{i}\right|_{0}=\max _{1 \leq i \leq m}\left|a_{i}\right| \leq \max _{1 \leq i \leq n}\left|a_{i}\right|=\left|\sum_{i=1}^{n} a_{i} e_{i}\right|_{0}$.

Suponhamos que $\left|\sum_{i=1}^{m_{m}} a_{i} e_{i}\right|_{k} \leq\left|\sum_{i=1}^{n} a_{i} e_{i}\right|_{k}, \forall\left(a_{n}\right)_{n}$ e $m<n$. Provaremos que 
$\left|\sum_{i=1}^{m} a_{i} e_{i}\right|_{k+1} \leq\left|\sum_{i=1}^{n} a_{i} e_{i}\right|_{k+1}$

$\left|\sum_{i=1}^{m} a_{i} e_{i}\right|_{k+1}=\max \frac{1}{f(l)} \sum_{j=1}^{l}\left|E_{j}\left(\sum_{i=1}^{m} a_{i} e_{i}\right)\right|_{k} \leq \max \frac{1}{f(l)} \sum_{j=1}^{l}\left|E_{j}\left(\sum_{i=1}^{n} a_{i} e_{i}\right)\right|_{k}$ pela hipótese de indução, mas max $\frac{1}{f(l)} \sum_{j=1}^{l}\left|E_{j}\left(\sum_{i=1}^{n} a_{i} e_{i}\right)\right|_{k}=\left|\sum_{i=1}^{n} a_{i} e_{i}\right|_{k+1}$.

Portanto $\left|\sum_{i=1}^{m} a_{i} e_{i}\right|_{k} \leq\left|\sum_{i=1}^{n} a_{i} e_{i}\right|_{k} \forall k, \forall\left(a_{n}\right)_{n}$ e $m<n$.

$\operatorname{Assim} \max _{k}\left|\sum_{i=1}^{m} a_{i} e_{i}\right|_{k} \leq \max _{k}\left|\sum_{i=1}^{n} a_{i} e_{i}\right|_{k}$, ou seja, $\left\|\sum_{i=1}^{m} a_{i} e_{i}\right\| \leq\left\|\sum_{i=1}^{n} a_{i} e_{i}\right\|$.

3) $\left(e_{i}\right)_{i}$ gera $T S$.

Demonstração análoga a do espaço de Schreier.

4) $\left(e_{i}\right)_{i}$ é 1-incondicional para TS.

Pelo Teorema 0.5 , basta verificarmos que

$$
\left\|\sum_{i \in A} a_{i} e_{i}\right\| \leq\left\|\sum_{i \in B} a_{i} e_{i}\right\|
$$

onde $A$ e $B$ são subconjuntos finitos dos naturais com $A \subset B$.

$\left|\sum_{i \in A} a_{i} e_{i}\right|_{0}=\max _{i \in A}\left|a_{i}\right| \leq \max _{i \in B}\left|a_{i}\right|=\left|\sum_{i \in B} a_{i} e_{i}\right|_{0}$.

Suponhamos que $\left|\sum_{i \in A} a_{i} e_{i}\right|_{k} \leq\left|\sum_{i \in B} a_{i} e_{i}\right|_{k}$, onde $A$ e $B$ são subconjuntos finitos dos naturais com $A \subset B$. Vamos demonstrar que $\left|\sum_{i \in A} a_{i} e_{i}\right|_{k+1} \leq\left|\sum_{i \in B} a_{i} e_{i}\right|_{k+1}$.

$\left|\sum_{i \in A} a_{i} e_{i}\right|_{k+1}=\max \frac{1}{f(l)} \sum_{j=1}^{l}\left|E_{j}\left(\sum_{i \in A} a_{i} e_{i}\right)\right|_{k} \leq \max \frac{1}{f(l)} \sum_{j=1}^{l}\left|E_{j}\left(\sum_{i \in B} a_{i} e_{i}\right)\right|_{k}$ pela hipótese de indução, mas max $\frac{1}{f(l)} \sum_{j=1}^{l}\left|E_{j}\left(\sum_{i \in B} a_{i} e_{i}\right)\right|_{k}=\left|\sum_{i \in B} a_{i} e_{i}\right|_{k+1}$.

Portanto $\left|\sum_{i \in A} a_{i} e_{i}\right|_{k} \leq\left|\sum_{i \in B} a_{i} e_{i}\right|_{k}, \forall k$, onde $A$ e $B$ são subconjuntos finitos dos naturais $\operatorname{com} A \subset B$. Logo $\max _{k}\left|\sum_{i \in A} a_{i} e_{i}\right|_{k} \leq \max _{k}\left|\sum_{i \in B} a_{i} e_{i}\right|_{k}$, ou seja, $\left\|\sum_{i \in A} a_{i} e_{i}\right\| \leq\left\|\sum_{i \in B} a_{i} e_{i}\right\|$, onde $A$ e $B$ são subconjuntos finitos dos naturais $\operatorname{com} A \subset B$. 
Segue de 1), 2), 3) e 4) que $\left(e_{i}\right)_{i}$ é base 1-incondicional para TS.

b) Vamos primeiro fazer a demonstração para $x \in c_{00}$.

Se $\|x\|=|x|_{0}$, segue que para todo $l \geq 2$ e subconjuntos finitos $E_{1}, E_{2}, \ldots, E_{l} \operatorname{dos} \mathbb{N}$ $\operatorname{com} E_{1}<E_{2}<\ldots<E_{l}, \frac{1}{f(l)} \sum_{i=1}^{l}\left\|E_{i}(x)\right\|=\max _{k \geq 0} \frac{1}{f(l)} \sum_{i=1}^{l}\left|E_{i}(x)\right|_{k} \leq$ $\max _{k \geq 0} \max _{l \geq 2} \frac{1}{f(l)} \sum_{i=1}^{l}\left|E_{i}(x)\right|_{k} \leq \max _{k \geq 0}|x|_{k+1}=\max _{k \geq 1}|x|_{k} \leq\|x\|$.

Portanto para qualquer $l \geq 2, \frac{1}{f(l)} \sum_{i=1}^{l}\left\|E_{i}(x)\right\| \leq\|x\|$ logo $\sup _{l \geq 2} \frac{1}{f(l)} \sum_{i=1}^{l}\left\|E_{i}(x)\right\| \leq\|x\|$.

Se $\|x\|=|x|_{k}>|x|_{k-1} \geq|x|_{0}$, para algum $k \geq 1$. existem $l \in \mathbb{N}, l \geq 2$, subconjuntos finitos do $\mathbb{N}, E_{1}, E_{2}, \ldots, E_{l}$ com $E_{1}<E_{2}<\ldots<E_{l}$ tal que $\|x\|=|x|_{k}=$ $\frac{1}{f(l)} \sum_{i=1}^{l}\left|E_{i}(x)\right|_{k-1} \leq \frac{1}{f(l)} \sum_{i=1}^{l}\left\|E_{i}(x)\right\| \leq \sup _{\bar{l} \geq 2} \frac{1}{f(\bar{l})} \sum_{i=1}^{i}\left\|\bar{E}_{i}(x)\right\|$.

Temos também que existe $l^{\prime} \in \mathbb{N}, l^{\prime} \geq 2$, subconjuntos finitos do $\mathbb{N}, E_{1}^{\prime}, E_{2}^{\prime}, \ldots, E_{l^{\prime}}^{\prime}$ com $E_{1}^{\prime}<E_{2}^{\prime}<\ldots<E_{l^{\prime}}^{\prime}$ e um $k^{\prime} \in \mathbb{N}$ tal que $\sup _{\bar{l} \geq 2} \frac{1}{f(\bar{l})} \sum_{i=1}^{i}\left\|\bar{E}_{i}(x)\right\|=\frac{1}{f\left(l^{\prime}\right)} \sum_{i=1}^{l^{\prime}}\left\|E_{i}^{\prime}(x)\right\|=$ $\frac{1}{f\left(l^{\prime}\right)} \sum_{i=1}^{l^{\prime}}\left|E_{i}^{\prime}(x)\right|_{k^{\prime}} \leq|x|_{k^{\prime}+1} \leq|x|_{k}=\|x\|$. Portanto $\|x\|=\sup _{\bar{l} \geq 2} \frac{1}{f(\bar{l})} \sum_{i=1}^{i}\left\|\bar{E}_{i}(x)\right\|$.

Logo $\|x\|=\max \left\{|x|_{0}, \sup _{l \geq 2} \frac{1}{f(l)} \sum_{i=1}^{l}\left\|E_{i}(x)\right\|\right\}$ para $x \in c_{00}$.

A demonstração de que o ítem b) desta Proposição vale para todo $x \in T S$ é análoga a demonstração feita para o espaço de Tsirelson.

Observação 3.6 Como a base $\left(e_{i}\right)_{i}$ de $c_{00}$ é 1-incondicional nesta norma, não há diferença se nós assumirmos que todas as sequências $E_{1}<\ldots<E_{N}$ são sequências de intervalos.

De fato, consideremos $\left\{E_{j}\right\}_{j=1}^{n}$ subconjuntos dos naturais. Se $E_{j}$ não é um intervalo, então vamos substituí-lo pelo menor intervalo que o contém, vamos chamar este intervalo de $\overline{E_{j}}$. Então $E_{j} \subset \overline{E_{j}}$ para qualquer $j, 1 \leq j \leq n$.

Como $\left(e_{i}\right)_{i}$ é 1-incondicional na norma de Schlumprecht temos $\left\|E_{j}(x)\right\| \leq\left\|\overline{E_{j}}(x)\right\|$. 
Sendo assim $\frac{1}{f(n)} \sum_{j=1}^{n}\left\|E_{j}(x)\right\| \leq \frac{1}{f(n)} \sum_{j=1}^{n}\left\|\overline{E_{j}}(x)\right\|$, e como para formar a norma do espaço consideramos $\sup _{n \geq 2} \frac{1}{f(n)} \sum_{i=1}^{n}\left\|E_{i}(x)\right\|$ podemos tomar todos os subconjuntos como sendo intervalos.

Nós agora adotaremos a convenção de que todas as sequências mencionadas são sequências de intervalos.

Para os próximos dois Lemas necessitamos de algumas definições introduzidas por Gowers e Maurey.

Definição 3.7 Seja $X$ o conjunto dos espaços normados da forma $X=\left(c_{00},\|\|.\right)$ tal que $\left(e_{i}\right)_{i}$ é uma base normalizada de $X$. Se $f \in \mathcal{F}, X \in X$ e todo $x \in X$ satisfaz a desigualdade

$$
\|x\| \geq \sup \left\{f(N)^{-1} \sum_{i=1}^{N}\left\|E_{\mathrm{i}}(x)\right\|: N \in \mathbb{N}, E_{1}<\ldots<E_{N}\right\}
$$

dizemos que $X$ satifaz uma $f$-estimativa inferior.

Note que esta definição implica que $\|E x\| \leq\|x\|$ para todo intervalo $E$ e vetor $x$, pois basta fazermos $N=1$ e $E_{1}=E$ onde $E$ é o intervalo desejado.

Definição 3.8 Dado um vetor $x \in X$ e um espaço $X \in X$, dizemos que $x$ é um $l_{1+}^{n}$ average com constante $C$ se $\|x\|=1$ e $x=\sum_{i=1}^{n} x_{i}$ para alguma sequência $x_{1}<\ldots .<x_{n}$ de elementos não nulos de $X$ tal que $\left\|x_{i}\right\| \leq C n^{-1}$ para cada $i$.

Definição 3.9 Um $l_{1+}^{n}$-vetor é qualquer multiplo positivo de um $l_{1+}^{n}$-average. Em outras palavras, um vetor $x$ é um $l_{1+}^{n}$-vetor com constante $C$ se ele pode ser escrito como $x=$ $x_{1}+\ldots+x_{n}$, onde $x_{1}<\ldots<x_{n}, x_{i}$ não nulos, $e\left\|x_{i}\right\| \leq C n^{-1}\|x\|$ para todo $i$.

Definição 3.10 Um subespaço de bloco de um espaço $X \in X$ é um subespaço gerado por uma base de bloco.

Lema 3.11 Sejam $f \in \mathcal{F}$ e $X \in X$ satisfazendo uma $f$-estimativa inferior. Então, para todo $n \in \mathbb{N}$ e todo $C>1$, todo subespaço de bloco $Y$ de $X$ contém um $l_{1+}^{n}$-averege com constante $C$. 


\section{Demonstração:}

Suponhamos que o resultado é falso. Seja $k$ um inteiro tal que $k \log C>\log f\left(n^{k}\right) . k$ existe porque $k \log C>\log f\left(n^{k}\right) \Longleftrightarrow \log C^{k}>\log f\left(n^{k}\right) \Longleftrightarrow C^{k}>f\left(n^{k}\right) \Longleftrightarrow \frac{f\left(n^{k}\right)}{C^{k}}<1$.

Pela propriedade 3 ) de $\mathcal{F}$ temos que $\lim _{x \rightarrow+\infty} \frac{f(x)}{x^{q}}=0, \forall q>0$. Seja $q$ tal que $C=n^{q}$, logo $\lim _{k \rightarrow+\infty} \frac{f\left(n^{k}\right)}{\left(n^{q}\right)^{k}}=0$ e portanto para $k$ suficientemente grande temos $\frac{f\left(n^{k}\right)}{\left(n^{q}\right)^{k}}<1$.

Sejam $N=n^{k}, x_{1}<\ldots<x_{N}$ uma sequência de vetores sucessivos de norma $1 \mathrm{em}$ $Y$ e $x=\sum_{i=1}^{N} x_{i}$. Para todo $0 \leq i \leq k$ e todo $1 \leq j \leq n^{k-i}$, seja $x(i, j)=\sum_{t=(j-1) n^{i}+1}^{j n^{i}} x_{t}$. Assim $x(0, j)=x_{j}, x(k, 1)=x$ e para $1 \leq i \leq k$ cada $x(i, j)$ é a soma de $n$ sucessivos $x(i-1, j)^{\prime} s$. Vamos verificar esta afirmação. Seja $1 \leq r \leq k$.

Para $i=r$, temos $1 \leq j \leq n^{k-r}$.

$x(r, 1)=\sum_{t=1}^{n^{r}} x_{t}=\sum_{t=1}^{n^{r-1}} x_{t}+\sum_{t=n^{r-1}+1}^{2 n^{r-1}} x_{t}+\ldots+\sum_{t=(n-1) n^{r-1}+1}^{n n^{r-1}} x_{t}$, mas

$x(r-1,1)=\sum_{t=1}^{n^{r-1}} x_{t}, x(r-1,2)=\sum_{t=n^{r-1}+1}^{2 n^{r-1}} x_{t}, \ldots, x(r-1, n)=\sum_{t=(n-1) n^{r-1}+1}^{n n^{r-1}} x_{t}$, onde os $x(r-1, j)^{\prime} s$ foram obtidos da fórmula $x(i, j)=\sum_{t=(j-1) n^{\prime}+1}^{j n^{\prime}} x_{t}$.

Portanto $x(r, 1)=x(r-1,1)+x(r-1.2)+\ldots .+x(r-1, n)$.

$x(r, 2)=\sum_{t=n^{r}+1}^{2 n^{r}} x_{t}=\sum_{t=n^{r}+1}^{n^{r}+n^{r-1}} x_{t}+\sum_{t=n^{r}+n^{r-1}+1}^{n^{r}+2 n^{r-1}} x_{t}+\ldots .+\sum_{t=2 n^{r}-n^{r-1}+1}^{2 n^{r}} x_{t}$, mas $x(r-1, n+1)=\sum_{t=n^{r}+1}^{n^{r}+n^{r-1}} x_{t}, x(r-1, n+2)=\sum_{t=n^{r}-n^{r-1}+1}^{n^{r}+2 n^{r-1}} x_{t}, \ldots, x(r-1,2 n)=\sum_{t=2 n^{r}-n^{r-1}+1}^{2 n^{r}} x_{t}$.

Portanto $x(r, 2)=x(r-1, n+1)+x(r-1, n+2)+\ldots+x(r-1,2 n)$.

Calculando para um $j$ qualquer tal que $1 \leq j \leq n^{k-r}$ temos, $x(r, j)=\sum_{t=(j-1) n^{r}+1}^{j n^{r}} x_{t}=\sum_{t=(j-1) n^{r}+1}^{(j-1) n^{r}+n^{r-1}} x_{t}+\sum_{t=(j-1) n^{r}+n^{r-1}+1}^{(j-1) n^{r}+2 n^{r-1}} x_{t}+\ldots .+\sum_{t=(j-1) n^{r}+(n-1) n^{r-1}+1}^{(j-1) n^{r}+n n^{r-1}} x_{t}$,
$x(r-1,(j-1) n+1)=\sum_{t=(j-1) n^{r}+1}^{(j-1) n^{r}+n^{r-1}} x_{t}, x(r-1,(j-1) n+2)=\sum_{t=(j-1) n^{r}+n^{r-1}+1}^{(j-1) n^{r}+2 n^{r-1}} x_{t}, \ldots .$. 


$$
x(r-1, j n)=\sum_{t=j n^{r}-n^{r-1}+1}^{j n^{r}} x_{t} .
$$

Portanto $x(r, j)=x(r-1,(j-1) n+1)+x(r-1,(j-1) n+2)+\ldots+x(r-1, j n)$.

Logo para $1 \leq i \leq k$ temos que $x(i, j)$ é a soma de $n$ sucessivos $x(i-1, j)^{\prime} s$.

Pelo que supomos nenhum $x(i, j)$ é um $l_{1+}^{n}$-vetor com constante $C$, pois a soma dos n sucessivos $x(i-1, j)^{\prime}$ s não é um $l_{1+}^{n}$-average com constante $C$. Então temos que $\|x(i-1, j)\| \geq C n^{-1}\|x(i, j)\|$, logo $\|x(i, j)\| \leq\|x(i-1, j)\| C^{-1} n$.

Vamos provar agora por indução que $\|x(i, j)\| \leq C^{-i} n^{i}$, em particular, $\|x\| \leq$ $C^{-k} n^{k}=C^{-k} N$.

Para $i=1,\|x(1, j)\| \leq\|x(0, j)\| C^{-1} n=\left\|x_{j}\right\| C^{-1} n$ logo $\|x(1, j)\| \leq C^{-1} n$.

Para $i=2,\|x(2, j)\| \leq\|x(1, j)\| C^{-1} n \leq C^{-1} n C^{-1} n$ logo $\|x(2, j)\| \leq C^{-2} n^{2}$.

Suponhamos que para $r$ vale $\|x(r, j)\| \leq C^{-r} n^{r}$. Vamos provar que vale para $r+1$. $\|x(r+1, j)\| \leq\|x(r, j)\| C^{-1} n \leq C^{-r} n^{r} C^{-1} n \operatorname{logo}\|x(r+1, j)\| \leq C^{-r-1} n^{r+1}$.

Portanto $\|x(i, j)\| \leq C^{-i} n^{i}$ para $i, 1 \leq i \leq k$.

$X$ satisfaz uma $f$-estimativa inferior então $\|x\| \geq \sup \left\{f(N)^{-1} \sum_{i=1}^{N}\left\|E_{i}(x)\right\|: N \in\right.$ $\left.\mathbb{N}, E_{1}<\ldots<E_{N}\right\}$. Considerando $E_{i}=\operatorname{ran}\left(x_{i}\right)$ temos $\|x\| \geq f(N)^{-1}\left(\left\|E_{1}\left(x_{1}\right)\right\|+\ldots+\right.$ $\left.\left\|E_{N}\left(x_{N}\right)\right\|\right)=f(N)^{-1}\left(\left\|x_{1}\right\|+\ldots+\left\|x_{N}\right\|\right)=f(N)^{-1} N$. Portanto $\|x\| \geq f(N)^{-1} N$.

Logo $f(N)^{-1} N \leq\|x\| \leq C^{-k} N$, ou seja, $f(N)^{-1} N \leq C^{-k} N$ então $f(N)^{-1} \leq C^{-k}$. Contradição, pois pela escolha de $k$ temos que $C^{k}>f(N) \operatorname{logo} C^{-k}<f(N)^{-1}$.

Observação 3.12 Pelo Lema acima temos que se $n \in \mathbb{N}$ e $C>1$ considerando $k$ um inteiro tal que $k \log C>\log f\left(n^{k}\right)$ e tomando $N=n^{k}$ temos que se $x_{1}<x_{2}<\ldots<x_{N}$ é uma sequência de sucessivos vetores de norma 1 de $X$, então existe $\left(x_{11}, \ldots, x_{1 n}\right)$ tal que $\sum_{i=1}^{n} x_{1 i}$ é um $l_{1+}^{n}$-average com constante $C$.

Lema 3.13 Sejam $M, N \in \mathbb{N}, C \geq 1, f \in \mathcal{F}, X \in X$ satisfazendo uma $f$ - estimativa inferior, $x \in X$ um $l_{1+}^{N}$-vetor com constante $C$, e $E_{1}<\ldots<E_{M}$ uma sequência de 
intervalos. Então $\sum_{j=1}^{M}\left\|E_{j} x\right\| \leq C(1+2 M / N)\|x\|$.

\section{Demonstração:}

Por conveniência, vamos normalizar $x$ tal que $\|x\|=N$ e $x=\sum_{i=1}^{N} x_{i}$, onde $x_{1}<$ $\ldots<x_{N}$ e $\left\|x_{i}\right\| \leq C$. Dado $j$, seja $A_{j}=\left\{1 \leq i \leq N: \operatorname{supp}\left(x_{i}\right) \subset E_{j}\right\}$ e $B_{j}=\{1 \leq i \leq$ $\left.N: E_{j}\left(x_{i}\right) \neq 0\right\}$, temos que $A_{j} \subset B_{j}, 1 \leq j \leq M$.

Pela desigualdade triangular temos, $\left\|E_{j} x\right\|=\left\|E_{j}\left(x_{1}+x_{2}+\ldots+x_{N}\right)\right\|=\left\|E_{j}\left(x_{1}\right)+\ldots+E_{j}\left(x_{N}\right)\right\|=\left\|\sum_{i \in B} E_{j}\left(x_{i}\right)\right\| \leq \sum_{i \in B}\left\|E_{j}\left(x_{i}\right)\right\| \leq$ $\sum_{i \in B_{j}}\left\|x_{i}\right\| \leq C\left(\left|B_{j}\right|\right) \leq C\left(\left|A_{j}\right|+2\right)$, pois temos que $\left|A_{j}\right|=\left|B_{j}\right|$ ou $\left|B_{j}\right|=\left|A_{j}\right|+1$ ou $\left|B_{j}\right|=\left|A_{j}\right|+2$.

Visto que $\sum_{j=1}^{M}\left|A_{j}\right|=\left(\left|A_{1}\right|+\ldots+\left|A_{M}\right|\right) \leq N$, obtemos $\sum_{j=1}^{M}\left\|E_{j} x\right\| \leq C(N+2 M)$ o que nos dá o resultado pela nossa normalização.

Para continuarmos será preciso introduzir mais duas definições.

Definição 3.14 Se $f \in \mathcal{F}$, seja $M_{f}: \mathbb{R} \rightarrow \mathbb{R}$ definida por $M_{f}=f^{-1}\left(36 x^{2}\right)$. Dizemos que uma sequência $x_{1}<\ldots<x_{N}$ é uma sequência rapidamente crescente de $l_{1+\text {-average, ou }}$ $R I S$, para $f$ de comprimento $N$ com constante $1+\epsilon$ se $x_{k}$ é um $l_{1+}^{n_{k}}$-average com constante $1+\epsilon$ para cada $k, n_{1} \geq 2(1+\epsilon) M_{f}\left(N / \epsilon^{\prime}\right) / \epsilon^{\prime} f^{\prime}(1)$ e $\frac{\epsilon^{\prime}}{2} f\left(n_{k}\right)^{1 / 2} \geq\left|\operatorname{ran}\left(x_{k-1}\right)\right|$ para cada $k=2, \ldots, N$.

Aqui $f^{\prime}(1)$ é a derivada a direita de $f$ em 1 e $\epsilon^{\prime}$ é a notação usual para $\min \{\epsilon, 1\}$.

Um ponto importante é que os $n_{k^{\prime} s}$ aumentam bastante, a velocidade depende do tamanho do ran dos $x_{j^{\prime} s}$ anteriores. Algumas vezes será conveniente chamar um vetor de um vetor-RIS se ele é um multiplo não nulo da soma de uma RIS.

Definição 3.15 Um funcional $x^{*}$ é uma $(M, g)$-forma se $\left\|x^{*}\right\| \leq 1$ e $x^{*}=\sum_{j=1}^{M} x_{j}^{*}$ para alguma sequência $x_{1}^{*}<\ldots<x_{M}^{*}$ de funcionais sucessivos tal que $\left\|x_{j}^{*}\right\| \leq g(M)^{-1}$ para cada $j$. 
Lema 3.16 Sejam $f, g \in \mathcal{F}, g \geq f^{1 / 2}, X \in X$ satisfazendo uma $f$-estimativa inferior, $\epsilon>0, x_{1}, \ldots, x_{N}$ uma RIS em $X$ para $f$ com constante $1+\epsilon, x=\sum_{i=1}^{N} x_{i}, M \geq M_{f}\left(N / \epsilon^{\prime}\right)$, $x^{*}$ uma $(M, g)$-forma e E um intervalo qualquer. Então $\left|x^{*}(E x)\right| \leq 1+\epsilon+\epsilon^{\prime}$.

\section{Demonstração:}

Temos que $x^{*}(E x)=\left(E x^{*}\right)(x)$, pois sejam $E_{1}=\operatorname{supp}\left(x_{1}^{*}\right), E_{2}=\operatorname{supp}\left(x_{2}^{*}\right), \ldots, E_{M}=$ $\operatorname{supp}\left(x_{M}^{*}\right), I_{1}=\operatorname{supp}\left(x_{1}\right), I_{2}=\operatorname{supp}\left(x_{2}\right), \ldots, I_{N}=\operatorname{supp}\left(x_{N}\right)$ e consideremos $F=\cup E_{\mathbf{i}}$, $1 \leq i \leq M$ e $I=\cup I_{i}, 1 \leq i \leq N$.

Então $x^{*}=\left(a_{1}, a_{2}, \ldots, a_{n}, \ldots\right)$ onde $a_{i} \neq 0$ se $i \in F$ e $x=\left(b_{1}, b_{2}, \ldots, b_{n}, \ldots.\right)$ onde $b_{i} \neq 0$ se $i \in I$. Logo $x^{*}(x)=\sum_{i=1}^{\infty} a_{i} b_{i}$, então $x^{*}(E x)=\sum_{i \in E} a_{i} b_{i}=\left(E x^{*}\right)(x)$.

Se $x^{*}$ é uma (M,g)-forma então $E x^{*}$ também é, para qualquer intervalo $E$, pois $\left\|E x^{*}\right\| \leq\left\|x^{*}\right\| \leq 1, E x^{*}=E\left(\sum_{j=1}^{M} x_{j}^{*}\right)=\sum_{j=1}^{M} E x_{j}^{*}$, onde como $x_{1}^{*}<\ldots<x_{M}^{*}$ também temos $E x_{1}^{*}<\ldots<E x_{M}^{*}$ e $\left\|E x_{j}^{*}\right\| \leq\left\|x_{j}^{*}\right\| \leq g(M)^{-1}$ para cada $j$.

Para cada $i$ seja $n_{i}$ o máximo tal que $x_{i}$ é um $l_{1+}^{n_{i}}$-average com constante $1+\epsilon$. Por hipótese $x^{*}=\sum_{j=1}^{M} x_{j}^{*}$, onde $x_{1}^{*}<\ldots<x_{M}^{*}$. Consideremos $E_{j}=\operatorname{ran}\left(x_{j}^{*}\right)$, vamos obter três estimativas para $\left|x^{*}\left(x_{i}\right)\right|$.

1) Como $\left\|x^{*}\right\| \leq 1$ temos que $\left|x^{*}\left(x_{i}\right)\right| \leq\left\|x^{*}\right\|\left\|x_{i}\right\| \leq 1$, pois $\left\|x_{i}\right\|=1$.

2) Sabendo que $\left\|x_{j}^{*}\right\| \leq g(M)^{-1} \leq f(M)^{-1 / 2}$ temos que $\left|x^{*}\left(x_{i}\right)\right|=\left|\sum_{j=1}^{M} x_{j}^{*}\left(x_{i}\right)\right| \leq$ $\sum_{j=1}^{M}\left|x_{j}^{*}\left(x_{i}\right)\right|=\sum_{j=1}^{M}\left|\left(E_{j} x_{j}^{*}\right)\left(x_{i}\right)\right|=\sum_{j=1}^{M}\left|x_{j}^{*}\left(E_{j} x_{i}\right)\right| \leq \sum_{j=1}^{M}\left\|x_{j}^{*}\right\|\left\|E_{j} x_{i}\right\| \leq \sum_{j=1}^{M} f(M)^{-1 / 2}\left\|E_{j} x_{i}\right\|=$ $f(M)^{-1 / 2} \sum_{j=1}^{M}\left\|E_{j} x_{i}\right\|$.

Pelo Lema 3.13 temos $\sum_{j=1}^{M}\left\|E_{j} x_{i}\right\| \leq(1+\epsilon)\left(1+2 M n_{i}^{-1}\right)$, portanto $\left|x^{*}\left(x_{i}\right)\right| \leq f(M)^{-1 / 2}(1+$ $\epsilon)\left(1+2 M n_{i}^{-1}\right)$. 
3) Pela nossa hipótese sobre $X$ temos $f(n)\|x\| \geq \sum_{i=1}^{n}\left\|F_{i}(x)\right\|, \forall n \in \mathbb{N}$, onde os $F_{i^{\prime} s}$ são intervalos de inteiros consecutivos. Consideremos $A=\left\{j: E_{j}\left(x_{i}\right) \neq 0\right\} \operatorname{logo}$ $\sum_{j=1}^{M}\left\|E_{j}\left(x_{i}\right)\right\|=\sum_{j \in A}\left\|E_{j}\left(x_{i}\right)\right\| \leq f(|A|) \leq f\left(\left|\operatorname{ran}\left(x_{i}\right)\right|\right.$, pois $|A| \leq\left|\operatorname{supp}\left(x_{i}\right)\right| \leq\left|\operatorname{ran}\left(x_{i}\right)\right|$.

Seja $t$ o maior índice tal que $n_{t} \leq M$ e consideremos $i \leq t-1$. Sabemos que $\left|\operatorname{supp}\left(x_{j}\right)\right| \geq n_{j}$, já que $x_{j}$ é um $l_{1+}^{n_{j}}$-average, logo $\left|\operatorname{ran}\left(x_{j}\right)\right| \geq n_{j}$.

Pela definição de RIS temos que $\left|\operatorname{ran}\left(x_{k-1}\right)\right| \leq \frac{\epsilon^{\prime}}{2} f\left(n_{k}\right)^{1 / 2}$, logo $\left|\operatorname{ran}\left(x_{t-2}\right)\right| \leq \frac{\epsilon^{\prime}}{2} f\left(n_{t-1}\right)^{1 / 2} \leq \frac{1}{2} f\left(n_{t-1}\right) \leq \frac{1}{2} f\left(\left|\operatorname{ran}\left(x_{t-1}\right)\right|\right)$, então $f\left(\left|\operatorname{ran}\left(x_{t-2}\right)\right|\right) \leq$ $\left|\operatorname{ran}\left(x_{t-2}\right)\right| \leq \frac{1}{2} f\left(\left|\operatorname{ran}\left(x_{t-1}\right)\right|\right)$. $\left|\operatorname{ran}\left(x_{t-3}\right)\right| \leq \frac{\epsilon}{2} f\left(n_{t-2}\right)^{1 / 2} \leq \frac{1}{2} f\left(n_{t-2}\right) \leq \frac{1}{2} f\left(\left|\operatorname{ran}\left(x_{t-2}\right)\right|\right) \leq \frac{1}{2^{2}} f\left(\left|\operatorname{ran}\left(x_{t-1}\right)\right|\right)$ então $f\left(\left|\operatorname{ran}\left(x_{t-3}\right)\right|\right) \leq\left|\operatorname{ran}\left(x_{t-3}\right)\right| \leq \frac{1}{2^{2}} f\left(\left|\operatorname{ran}\left(x_{t-1}\right)\right|\right)$.

Generalizando temos $f\left(\left|\operatorname{ran}\left(x_{i}\right)\right|\right) \leq 2^{i-t+1} f\left(\left|\operatorname{ran}\left(x_{t-1}\right)\right|\right)$. (II)

Temos também que $f\left(\left|\operatorname{ran}\left(x_{t-1}\right)\right|\right) \leq\left|\operatorname{ran}\left(x_{t-1}\right)\right| \leq \frac{\epsilon^{\prime}}{2} f\left(n_{t}\right)^{1 / 2} \leq \frac{\epsilon^{\prime}}{2} f(M)^{1 / 2}$.

Consequentemente $\left|x^{*}\left(x_{i}\right)\right| \leq f(M)^{-1 / 2} \sum_{j=1}^{M}\left\|E_{j} x_{i}\right\| \stackrel{(I)}{\leq} f(M)^{-1 / 2} f\left(\left|\operatorname{ran}\left(x_{i}\right)\right|\right) \stackrel{(I I)}{\leq}$ $f(M)^{-1 / 2} 2^{i-t+1} f\left(\left|\operatorname{ran}\left(x_{t-1}\right)\right|\right) \stackrel{(I I I)}{\leq} f(M)^{-1 / 2} 2^{i-t+1} \frac{\epsilon^{\prime}}{2} f(M)^{1 / 2}=2^{i-t+1} \frac{\epsilon^{\prime}}{2}$ para $i \leq t-1$. Por 1), 2) e 3) temos, $\left|x^{*}(x)\right|=\left|x^{*}\left(\sum_{i=1}^{N} x_{i}\right)\right| \leq \sum_{i=1}^{N}\left|x^{*}\left(x_{i}\right)\right|=\sum_{i=1}^{t-1}\left|x^{*}\left(x_{i}\right)\right|+\left|x^{*}\left(x_{t}\right)\right|+\sum_{j=t+1}^{N}\left|x^{*}\left(x_{j}\right)\right| \leq \sum_{i=1}^{t-1} 2^{i-t+1} \frac{\epsilon^{\prime}}{2}+$
$+1+\sum_{j=t+1}^{N} f(M)^{-1 / 2}(1+\epsilon)\left(1+2 M n_{j}^{-1}\right)=\frac{\epsilon^{\prime}}{2} 2^{-t} 2 \sum_{i=1}^{t-1} 2^{i}+1+f(M)^{-1 / 2}(1+\epsilon) \sum_{j=t+1}^{N}\left(1+2 \frac{M}{n_{j}}\right)$.

Como $n_{t}$ é o máximo tal que $n_{t} \leq M$ temos que $n_{t+1} \geq M, \operatorname{logo} \frac{M}{n_{j}} \leq 1, \forall j \geq t+1$.

Então prosseguindo, $\frac{\epsilon^{\prime}}{2} 2^{-t} 2 \sum_{i=1}^{t-1} 2^{i}+1+f(M)^{-1 / 2}(1+\epsilon) \sum_{j=t+1}^{N}\left(1+2 \frac{M}{n_{j}}\right) \leq \epsilon^{\prime} \underbrace{2^{-t} \sum_{i=1}^{t-1} 2^{i}}_{\leq 1}+1+f(M)^{-1 / 2}(1+\epsilon) 3(N-t) \leq$ $\epsilon^{\prime}+1+3(1+\epsilon)(N-t) f(M)^{-1 / 2}$.

Sabemos por hipótese que $M \geq M_{f}\left(N / \epsilon^{\prime}\right)$. ou seja $M \geq f^{-1}\left(36 \frac{N^{2}}{\left(\epsilon^{\prime}\right)^{2}}\right)$, consequentemente $f(M)^{-1 / 2} \leq \frac{\epsilon^{\prime}}{6 N}$ 
Substituindo na inequação acima obtemos $\epsilon^{\prime}+1+3(1+\epsilon)(N-t) f(M)^{-1 / 2} \leq \epsilon^{\prime}+$ $1+3(1+\epsilon) N\left(\frac{\epsilon^{\prime}}{6 N}\right)=1+\epsilon^{\prime}+\left(\epsilon^{\prime} / 2\right)(1+\epsilon) \leq 1+\epsilon^{\prime}+\epsilon$.

Portanto $\left|x^{*}(x)\right| \leq 1+\epsilon+\epsilon^{\prime}, \log 0\left|x^{*}(E x)\right| \leq 1+\epsilon+\epsilon^{\prime}$.

Corolário 3.17 Sejam $f, X, \epsilon, M, x_{1}, \ldots, x_{N}$ e $x$ como no Lema 3.16, e sejam $E_{1}<\ldots<$ $E_{M}$. Então $f(M)^{-1} \sum_{i=1}^{M}\left\|E_{\mathrm{i}} x\right\| \leq 1+\epsilon+\epsilon^{\prime}$.

\section{Demonstração:}

Primeiro vamos definir $x_{i}^{*}$ como sendo o funcional suporte de $E_{i} x, i=1,2, \ldots, M$.

Se $E_{i} x=0$, coloquemos $x_{i}^{*}=0$. Caso contrário seja $S_{i}^{*}:\left\langle\frac{E_{i} x}{\left\|E_{i} x\right\|}\right\rangle \rightarrow \mathbb{R}$ tal que $S_{i}^{*}\left(\alpha \frac{E_{i} x}{\left\|E_{i} x\right\|}\right)=\alpha$. $S_{i}^{*}$ é um funcional linear contínuo, pois consideremos $z=\alpha \frac{E_{i} x}{\left\|E_{i} x\right\|} \mathrm{e}$ $y=\beta \frac{E_{i} x}{\left\|E_{i} x\right\|}, \alpha \neq \beta$ e $\gamma \in \mathbb{R}$.

$S_{i}^{*}(z+y)=S_{i}^{*}\left(\frac{E_{i} x}{\left\|E_{\mathbf{t}} x\right\|}(\alpha+\beta)\right)=\alpha+\beta=S_{i}^{*}(z)+S_{i}^{*}(y)$.

$S_{i}^{*}(\gamma z)=S_{i}^{*}\left(\gamma \alpha \frac{E_{i} x}{\left\|E_{i} x\right\|}\right)=\gamma \alpha=\gamma S_{i}^{*}(z)$.

$\sup _{\|z\| \leq 1}\left\|S_{i}^{*}(z)\right\|=\sup _{\|z\| \leq 1}|\alpha| \leq 1$, pois $\|z\|=\left\|\alpha \frac{E_{i x}}{\left\|E_{i} x\right\|}\right\|=|\alpha| \leq 1$.

Portanto $S_{i}^{*}$ é um funcional linear contínuo com $\left\|S_{i}^{*}\right\|=1$.

Escrevendo $E_{i}=\left\{n_{i}, \ldots, n_{i}+m_{i}\right\}$, pelo Teorema de Hahn-Banach (veja [15]) existe

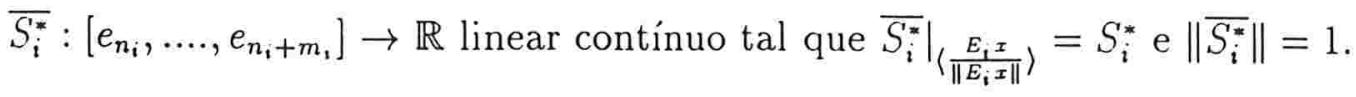

Agora vamos definir $x_{i}^{*}: X \rightarrow \mathbb{R}$ pondo $x_{i}^{*}(y)=\overline{S_{i}^{*}}\left(E_{i} y\right)$, para qualquer $y \in X$. Logo $x_{i}^{*}$ é um funcional linear contínuo tal que:

1) $x_{i}^{*}(x)=\overline{S_{i}^{*}}\left(E_{i} x\right)=\left\|E_{i} x\right\| \overline{S_{i}^{*}}\left(\frac{E_{i} x}{\left\|E_{i} x\right\|}\right)=\left\|E_{i} x\right\| S_{i}^{*}\left(\frac{E_{i} x}{\left\|E_{i} x\right\|}\right)=\left\|E_{i} x\right\|$.

2) $\operatorname{supp}\left(x_{i}^{*}\right) \subset E_{i}$.

3) $\left\|x_{i}^{*}\right\| \leq 1$, pois considere $y \in X$ com $\|y\| \leq 1$, logo $\left|x_{i}^{*}(y)\right|=\left|\overline{S_{i}^{*}}\left(E_{i} y\right)\right| \leq\left\|\overline{S_{i}^{*}}\right\|\left\|E_{i} y\right\| \leq$ 1.

4) Consideremos $x^{*}=f(M)^{-1} \sum_{i=1}^{M} x_{i}^{*}$, $x^{*}$ é uma (M, f)-forma. De fato, seja $y \in X$ com $\|y\| \leq 1$. Vamos calcular $\left|x^{*}(y)\right|$.

$\left|x^{*}(y)\right|=\left|f(M)^{-1} \sum_{i=1}^{M} x_{i}^{*}(y)\right| \leq f(M)^{-1} \sum_{i=1}^{M}\left|x_{i}^{*}(y)\right|=f(M)^{-1} \sum_{i=1}^{M}\left|\overline{S_{i}^{*}}\left(E_{i} y\right)\right| \leq$ 
$f(M)^{-1} \sum_{i=1}^{M}\left\|\overline{S_{i}^{*}}\right\|\left\|E_{i} y\right\|=f(M)^{-1} \sum_{i=1}^{M}\left\|E_{i} y\right\| \leq\|y\|$.

Portanto $\left\|x^{*}\right\| \leq 1$. Como $E_{1}<E_{2}<\ldots<E_{M}$ temos $x_{1}^{*}<x_{2}^{*}<\ldots<x_{M}^{*}$.

Temos também que $\left\|f(M)^{-1} x_{i}^{*}\right\|=f(M)^{-1}\left\|x_{i}^{*}\right\| \leq f(M)^{-1}$. Portanto $x^{*}$ é uma $(\mathrm{M}, \mathrm{f})$ forma.

Então temos $f(M)^{-1} \sum_{i=1}^{M}\left\|E_{i} x\right\|=f(M)^{-1} \sum_{i=1}^{M} x_{i}^{*}(x)=x^{*}(x)$, logo basta aplicar o Lema 3.16 com $f=g$ e teremos $f(M)^{-1} \sum_{i=1}^{M}\left\|E_{i} x\right\| \leq 1+\epsilon+\epsilon^{\prime}$.

Para continuarmos precisaremos da noção de comprimento de um intervalo E associado a uma RIS.

Definição 3.18 Sejam $x_{1}<\ldots<x_{N}$ uma sequência rapidamente crescente para $f$ com constante $1+\epsilon$ para alguma $f \in \mathcal{F}$ e algum $\epsilon>0$. Para cada $i$, seja $n_{i}$ o máximo tal que $x_{i}$ é um $l_{1+}^{n_{i}}$-average com constante $1+\epsilon$, vamos escrever isto como $x_{i}=x_{i 1}+\ldots+x_{i n_{i}}$ onde $\left\|x_{i j}\right\| \leq(1+\epsilon) n_{i}^{-1}$ para cada $j$.

Dado um intervalo $E \subset \mathbb{N}$, sejam $i=i_{E}$ e $j=j_{E}$ respectivamente o mínimo e o máximo tal que Ex $x_{i}$ e Ex $x_{j}$ são não nulos, e sejam $r=r_{E}$ e $s=s_{E}$ respectivamente o mínimo e o máximo tal que Ex $x_{i r}$ e Ex js são não nulos. Definamos o comprimento $\lambda(E)$ do intervalo $E$ por $j_{E}-i_{E}+\left(s_{E} / n_{j_{E}}\right)-\left(r_{E} / n_{i_{E}}\right)$.

Observação 3.19 Se $E_{1}<\ldots<E_{M}$ e $E=\cup_{i=1}^{M} E_{i}$ então $\sum_{i=1}^{M} \lambda\left(E_{i}\right) \leq \lambda(E)$.

De fato,

$\sum_{i=1}^{M} \lambda\left(E_{i}\right)=j_{E_{1}}-i_{E_{1}}+\left(s_{E_{1}} / n_{j_{E_{1}}}\right)-\left(r_{E_{1}} / n_{i_{E_{1}}}\right)+j_{E_{2}}-i_{E_{2}}+\left(s_{E_{2}} / n_{j_{E_{2}}}\right)-\left(r_{E_{2}} / n_{i_{E_{2}}}\right)+\ldots+j_{E_{M}}-$ $i_{E_{M}}+\left(s_{E_{M}} / n_{j_{E_{M}}}\right)-\left(r_{E_{M}} / n_{i_{E_{M}}}\right)=j_{E_{M}}-i_{E_{1}}+\left(s_{E_{M}} / n_{j_{E_{M}}}\right)-\left(r_{E_{1}} / n_{i_{E_{1}}}\right)+\sum_{k=1}^{M-1}\left(j_{E_{k}}-\right.$ $\left.i_{E_{k+1}}\right)+\sum_{k=1}^{M-1}\left(s_{E_{k}} / n_{j_{E_{k}}}-r_{E_{k+1}} / n_{i_{E_{k+1}}}\right)=\lambda(E)+\sum_{k=1}^{M-1}\left(j_{E_{k}}-i_{E_{k+1}}\right)+\sum_{k=1}^{M-1}\left(s_{E_{k}} / n_{j_{E_{k}}}+\right.$ $\left.r_{E_{k+1}} / n_{i_{E_{k+1}}}\right)$.

Seja $F=\left\{1 \leq k \leq M-1:\left(j_{E_{k}}-i_{E_{k+1}}\right) \neq 0\right\}$ então 


$$
\begin{aligned}
& \sum_{i=1}^{M} \lambda\left(E_{i}\right)=\lambda(E)+\sum_{k \notin F} \underbrace{\left(j_{E_{k}}-i_{E_{k+1}}\right)}_{=0}+\sum_{k \notin F} \underbrace{\left(s_{E_{k}} / n_{j_{E_{k}}}-r_{E_{k+1}} / n_{i_{E_{k+1}}}\right)}_{<0}+\sum_{k \in F} \underbrace{\left(j_{E_{k}}-i_{E_{k+1}}\right)}_{\leq-1}+ \\
& \sum_{k \in F} \underbrace{\left(s_{E_{k}} / n_{j_{E_{k}}}-r_{E_{k+1}} / n_{i_{E_{k+1}}}\right)}_{<1} . \\
& \quad \text { Portanto } \sum_{i=1}^{M} \lambda\left(E_{i}\right) \leq \lambda(E)+R-|F|+|F| \leq \lambda(E), \text { onde } R \text { é um número negativo. }
\end{aligned}
$$

Obviamente esta definição depende completamente da RIS, mas sempre estará claro no contexto que RIS estaremos considerando.

Lema 3.20 Dada uma RIS para $f$ com constante $1+\epsilon$, para alguma $f \in \mathcal{F}, 0<\epsilon \leq \frac{1}{2} e$ E um intervalo com $\lambda(E) \geq 1$ temos que $\|E x\| \geq \frac{1}{4}$, onde $x$ é a soma da RIS.

\section{Demonstração:}

Sejam $x_{1}, \ldots, x_{n}$ a RIS , $C=\left\{i: \operatorname{supp}\left(x_{i}\right) \cap E \neq \emptyset\right\}$ e $x=\sum_{i=1}^{n} x_{i}$.

Se $C=0$ então $\lambda(E)=0$.

Se $C=1$ então $0 \leq \lambda(E)<1$.

Se $C \geq 3$ então existe $x_{i} \subset E$ e $\lambda(E)>1$. Como $\|E x\| \geq\left\|x_{i}\right\|=1$ temos o resultado.

Se $C=2$, podemos ter,

1) $\lambda(E)>1$, os dois $x_{i^{\prime} s}$ estão contidos em $E$.

Neste caso temos $\|E x\| \geq\left\|x_{i}\right\|=1$, ou seja, $\|E x\| \geq \frac{1}{4}$.

2) $\lambda(E) \geq 1$, mas apenas um dos $x_{i^{\prime} s}$ está contido em $E$.

Análogo ao caso acima.

3) $\lambda(E) \geq 1$, mas não existe $j$ tal que $x_{j} \subset E$.

Lembrando sempre que os $x_{i^{\prime} s}$ são consecutivos temos,

$\lambda(E)=1+\frac{s_{E}}{n_{J_{E}}}-\frac{r_{E}}{n_{i_{E}}} \geq 1 \operatorname{logo} \frac{s_{E}}{n_{J_{E}}} \geq \frac{r_{E}}{n_{\mathrm{i}_{E}}}$.

caso 1: Se $\frac{r_{E}}{n_{i_{E}}}<\frac{1}{2}$

$1=\left\|x_{i_{E}}\right\| \leq\left\|x_{i_{E} 1}\right\|+\left\|x_{i_{E} 2}\right\|+\ldots+\left\|x_{i_{E} r_{E}}\right\|+\left\|x_{i_{E} r_{E}+1}+\ldots+x_{i_{E} n^{n_{E}}}\right\|$.

$1 \leq r_{E} \frac{(1+\varepsilon)}{n_{i_{E}}}+\left\|x_{i_{E} r_{E}+1}+\ldots+x_{i_{E} n_{n_{E}}}\right\|$. Como $\frac{r_{E}}{n_{i_{E}}}<\frac{1}{2}$ temos, 
$1 \leq \frac{(1+\epsilon)}{2}+\left\|x_{i_{E} r_{E}+1}+\ldots+x_{i_{E} n_{E}}\right\|$, ou seja, $1-\frac{(1+\epsilon)}{2} \leq\left\|x_{i_{E} r_{E}+1}+\ldots+x_{i_{E} n_{i_{E}}}\right\| \leq\|E x\|$.

Logo $\|E x\| \geq 1-\frac{(1+\epsilon)}{2}$. Basta então demonstrarmos que $1-\frac{(1+\epsilon)}{2} \geq \frac{1}{4}$.

$1-\frac{(1+\epsilon)}{2} \geq \frac{1}{4} \Longleftrightarrow \frac{(1-\epsilon)}{2} \geq \frac{1}{4} \Longleftrightarrow-4 \epsilon \geq-2 \Longleftrightarrow \epsilon \leq \frac{1}{2}$, que é o caso.

caso 2: Se $\frac{r_{E}}{n_{i_{E}}} \geq \frac{1}{2}$

Como $\frac{r_{E}}{n_{i_{E}}} \geq \frac{1}{2}$ temos que $\frac{s_{E}}{n_{J_{E}}} \geq \frac{1}{2}$, sabemos também que $\frac{s_{E}}{n_{J_{E}}}+\frac{n_{J_{E}}-s_{E}}{n_{\jmath_{E}}}=1$ logo $\frac{n_{J_{E}}-s_{E}}{n_{j_{E}}} \leq \frac{1}{2}$.

$1=\left\|x_{j_{E}}\right\| \leq\left\|x_{j_{E} 1}+\ldots+x_{j_{E} s_{E}}\right\|+\left\|x_{j_{E} s_{E}+1}\right\|+\ldots+\left\|x_{j_{E} \jmath_{E}}\right\|$.

$1 \leq\left\|x_{j_{E} 1}+\ldots+x_{j_{E} s_{E}}\right\|+\left(n_{j_{E}}-s_{E}\right) \frac{(1+\varepsilon)}{n_{j_{E}}}$. Como $\frac{n_{J_{E}}-s_{E}}{n_{j_{E}}} \leq \frac{1}{2}$ temos,

$1-\frac{(1+\epsilon)}{2} \leq\left\|x_{j_{E} 1}+\ldots+x_{j_{E} s_{E}}\right\| \leq\|E x\|$.

Portanto $\|E x\| \geq 1-\frac{(1+\epsilon)}{2}$ que já vimos que é maior ou igual a $\frac{1}{4}$ quando $\epsilon \leq \frac{1}{2}$.

Vamos observar também que $\|E x\|>\frac{1}{4}$ quando $0<\epsilon<\frac{1}{2}$ já que $1-\frac{(1+\epsilon)}{2}=\frac{1}{4}$ se, e somente se, $\epsilon=\frac{1}{2}$.

Lema 3.21 Sejam $f, g \in \mathcal{F}$ com $g \geq \sqrt{f}, X \in X$ satisfazendo uma $f$-estimativa inferior, $\epsilon>0, x_{1}<\ldots<x_{N}$ uma RIS em $X$ para $f$ com constante $1+\epsilon$, e $x=\sum_{i=1}^{N} x_{i}$. Suponha que $\|E x\| \leq \sup \left\{\left|x^{*}(E x)\right|: M \geq 2, x^{*}\right.$ é uma $(M, g)$-forma $\}$ para todo intervalo $E$ de comprimento maior ou igual a 1. Então $\|x\| \leq\left(1+\epsilon+\epsilon^{\prime}\right) N g(N)^{-1}$.

\section{Demonstração:}

Sejam $I=\left\{1 \leq i \leq N: x_{i} \subset E\right\} ; J=\left\{j \in \mathbb{N}: r_{E}+1 \leq j \leq n_{i_{E}}\right\}$ e $K=\{k \in \mathbb{N}:$ $\left.1 \leq k \leq s_{E}\right\}$.

Cada $x_{i}$ é um $l_{1+}^{n_{i}}$-average com constante $1+\epsilon$, ou seja, $x_{i}=x_{i 1}+\ldots+x_{i n_{i}}$ onde $\left\|x_{i j}\right\| \leq(1+\epsilon) n_{i}^{-1}$, para cada $j, 1 \leq j \leq n_{i}$, logo $\left\|x_{i}\right\|=\left\|\sum_{j=1}^{n_{i}} x_{i j}\right\| \leq \sum_{j=1}^{n_{i}}\left\|x_{i j}\right\| \leq$ $n_{i}(1+\epsilon) n_{i}^{-1}=1+\epsilon$. Como $i_{E} \geq 1$ temos $n_{i E} \geq n_{1}$ o que implica que $\frac{(1+\epsilon)}{n_{i E}} \leq \frac{(1+\epsilon)}{n_{1}}$. Vamos calcular $\|E x\|$.

$\|E x\|=\left\|\sum_{i \in I} x_{i}+\sum_{j \in J} x_{i_{E} j}+\sum_{k \in K} x_{j_{E} k}+x_{i_{E^{r}} E}\right\| \leq\left\|\sum_{i \in I} x_{i}\right\|+\left\|\sum_{j \in J} x_{i_{E} j}\right\|+\left\|\sum_{k \in K} x_{j_{E} k}\right\|+\left\|x_{i_{E} r_{E}}\right\| \leq$ $|I|(1+\epsilon)+|J| \frac{(1+\epsilon)}{n_{i_{E}}}+\left|K^{\prime}\right| \frac{(1+\epsilon)}{n_{j_{E}}}+\frac{(1+\epsilon)}{n_{i_{E}}} \leq(1+\epsilon) \lambda(E)+\frac{(1+\epsilon)}{n_{1}}=(1+\epsilon)\left(\lambda(E)+n_{1}^{-1}\right)$.

Se $\lambda(E) \geq \frac{(1+\epsilon)}{\epsilon^{\prime} n_{1}}$ então teremos que $(1+\epsilon) n_{1}^{-1} \leq \lambda(E) \epsilon^{\prime}$, logo obtemos que $\|E x\| \leq$ 
$\left(1+\epsilon+\epsilon^{\prime}\right) \lambda(E)$

Vamos definir $G$ por $G(x)=x$ quando $0 \leq x \leq 1$ e por $G(x)=x g(x)^{-1}$ quando $x \geq 1$. Como verificamos, no capítulo $2, G$ é concava e crescente sobre $[1,+\infty)$ e satisfaz $G(x y) \geq G(x) G(y)$ para todo $x, y$ no mesmo intervalo, e estas propriedades também são verdadeiras para todo $\mathbb{R}_{+}$.

Devemos mostrar que se $\lambda(E) \geq(1+\epsilon) / \epsilon^{\prime} n_{1}$, então $\|E x\| \leq\left(1+\epsilon+\epsilon^{\prime}\right) G(\lambda(E))$. Pela observação que fizemos sabemos que o resultado é verdadeiro quando $\lambda(E) \leq 1$.

Vamos supor então que $E$ é o menor intervalo de comprimento $\lambda(E) \geq(1+\epsilon) / \epsilon^{\prime} n_{1}$ para o qual temos $\|E x\| \geq\left(1+\epsilon+\epsilon^{\prime}\right) G(\lambda(E))$.

Sabemos que $\lambda(E) \geq 1$ e que existe alguma (M, g)-forma $x^{*}=\sum_{i=1}^{M} x_{i}^{*}$ tal que $\|E x\| \leq$ $\left|x^{*}(E x)\right|$, pois o supremo de $\left\{\left|x^{*}(E x)\right|: M \geq 2, x^{*}\right.$ é uma $(\mathrm{M}, \mathrm{g})$-forma $\}$ é assumido. Vejamos porque.

Temos que $x$ é a soma de uma RIS. seja $m$ um inteiro tal que $\operatorname{supp}(x) \subset[1, m]$. Seja $x^{*}$ uma $(M, g)$-forma $\operatorname{logo} x^{*}=\sum_{i=1}^{M} x_{i}^{*}$. então existe $y^{*}$ uma $(N, g)$-forma com $N \leq m \mathrm{e}$ $x^{*}(x)=y^{*}(x)$.

caso 1: $M \leq m$, nada a fazer.

caso 2: $\quad M \geq m$

Então $x^{*}(x)=\sum_{i=1}^{M} x_{i}^{*}(x)$. Seja $y^{*}$ definida por $y^{*}=\left.x^{*}\right|_{\left[e_{1}, \ldots, e_{m}\right]}$, logo $\left\|y^{*}\right\| \leq 1 \mathrm{e}$ $y^{*}(x)=\sum_{i \in A} x_{i}^{*}(x)$, onde $A=\left\{i: \operatorname{supp}\left(x_{i}^{*}\right) \cap[1, m] \neq 0\right\}$ e como $\left\|x_{i}^{*}\right\| \leq g(M)^{-1} \leq g(|A|)^{-1}$, segue que $y^{*}$ é uma $(|A|, g)$-forma.

Seja $D=\{(N, g)$-formas com $N \leq m\}$. Para cada p partição de $[1, m]=E_{1} \cup E_{2} \cup$ $\ldots \cup E_{N}$, consideremos $D_{p}$ o conjunto de funcionais $x^{*}$ satisfazendo 1 . $\left\|x^{*}\right\| \leq 1$ e 2 . $\left\|E_{\mathrm{i}}\left(x^{*}\right)\right\| \leq \frac{1}{g(N)}, \forall i, i=1, \ldots, N$.

Consideremos $G=\left\{x^{*}:\left\|x^{*}\right\| \leq 1\right\}$. Como as $E_{i^{\prime} s}$ são contínuas, segue que $F_{i}=$ $\left\{x^{*}:\left\|E_{\mathrm{i}} x^{*}\right\| \leq \frac{1}{g(N)}\right\}$ é fechado, $\forall i, i=1 \ldots N$.

Portanto $G \cap\left(\cap_{i=1}^{N} F_{i}\right)$ é compacto. Mas $x^{*} \in D \Longleftrightarrow x^{*} \in D_{p}$ para algum alguma 
p. Portanto $D=\cup D_{p}$, e como as partições de $[1, m]$ são em número finito segue que $D$ é compacto.

Se $M \geq M_{f}\left(N / \epsilon^{\prime}\right)$, então pelo Lema 3.16 temos $\left|x^{*}(E x)\right| \leq 1+\epsilon+\epsilon^{\prime}$. Como $\|E x\| \leq$ $\left|x^{*}(E x)\right|$ temos $\|E x\| \leq 1+\epsilon+\epsilon^{\prime} \leq\left(1+\epsilon+\epsilon^{\prime}\right) G(\lambda(E))$ já que $\lambda(E) \geq 1$, mas assumimos que $E$ satisfaz $\|E x\| \geq\left(1+\epsilon+\epsilon^{\prime}\right) G(\lambda(E))$ logo devemos ter $M \leq M_{f}\left(N / \epsilon^{\prime}\right) \leq \frac{n_{1} \epsilon^{\prime} f^{\prime}(1)}{2(1+\epsilon)}$, pela condição de RIS.

Colocando $E_{i}=E \cap \operatorname{ran}\left(x_{i}^{*}\right)$ e escrevendo $x$ em função dessas projeções temos $x=$ $E_{1} x+E_{2} x+\ldots+E_{M} x+y$ onde $y$ representa os pedaços de $x$ que não foram incluídos nas projeções. Então $E x=E_{1} x+E_{2} x+\ldots+E_{M} x+E y$ e $x^{*}(E x)=x^{*}\left(\sum_{i=1}^{M} E_{i} x+E y\right)=$ $\sum_{i=1}^{M} x^{*}\left(E_{i} x\right)+x^{*}(E y)$, onde $x^{*}(E y)=0$

$$
\begin{aligned}
& \text { Portanto }\|E x\| \leq\left|x^{*}(E x)\right|=\left|\sum_{i=1}^{M} x^{*}\left(E_{i} x\right)\right|=\left|\sum_{i=1}^{M} \sum_{j=1}^{M} x_{j}^{*}\left(E_{i} x\right)\right|=\left|\sum_{i=1}^{M} x_{i}^{*}\left(E_{i} x\right)\right| \leq \\
& \sum_{i=1}^{M}\left|x_{i}^{*}\left(E_{i} x\right)\right| \leq \sum_{i=1}^{M}\left\|x_{i}^{*}\right\|\left\|E_{i} x\right\| \leq g(M)^{-1} \sum_{i=1}^{M}\left\|E_{i} x\right\| .
\end{aligned}
$$

Seja $\lambda_{i}=\lambda\left(E_{i}\right)$ para cada $i$ e $\lambda(E)=\lambda$. Como $M \geq 2$ podemos assumir que nenhum dos intervalos $E_{i}$ são iguais a $E$, pois suponha $E_{1}=E$ então $\|E x\| \leq g(M)^{-1} \sum_{i=1}^{M}\left\|E_{i} x\right\|=$ $g(M)^{-1}\left\|E_{1} x\right\|=g(M)^{-1}\|E x\| \operatorname{logo} g(M) \leq 1$. Absurdo, pois $M \geq 2$ então $g(M)>1$.

Para cada $i$ devemos ter $\lambda_{i} \leq(1+\epsilon) / \epsilon^{\prime} n_{1}$ ou $\lambda_{i} \geq(1+\epsilon) / \epsilon^{\prime} n_{1}$ e neste caso, pela minimalidade de $E$, temos que $\left\|E_{i} x\right\| \leq\left(1+\epsilon+\epsilon^{\prime}\right) G\left(\lambda_{i}\right)$. Sejam $A$ o conjunto dos $i$ com a primeira propriedade, $B$ o complemento de $A$ e $k$ a cardinalidade de $A$.

É claro que $k<M$, pois se $k=M$ usando a definição de RIS teríamos $\|E x\| \leq$ $\leq g(M)^{-1} \sum_{i=1}^{M}\left\|E_{i} x\right\| \leq M\left(\lambda\left(E_{i}\right)(1+\epsilon)+\frac{(1+\epsilon)}{n_{1}}\right) \leq M\left((1+\epsilon) \frac{(1+\epsilon)}{\epsilon^{\prime} n_{1}}+\frac{(1+\epsilon)}{n_{1}}\right) \leq$ $\frac{n_{1} \epsilon^{\prime} f^{\prime}(1)}{2(1+\epsilon)}\left[\frac{(1+\epsilon)}{n_{1}}\left(\frac{(1+\epsilon)}{\epsilon^{\prime}}+1\right)\right]=\frac{\epsilon^{\prime} f^{\prime}(1)}{2}\left(\frac{1+\epsilon+\epsilon^{\prime}}{\epsilon^{\prime}}\right)=\frac{f^{\prime}(1)}{2}\left(1+\epsilon+\epsilon^{\prime}\right)<\left(1+\epsilon+\epsilon^{\prime}\right)$, mas sabemos que $E$ foi tomado tal que $\|E x\| \geq\left(1+\epsilon+\epsilon^{\prime}\right) G(\lambda(E)) \geq\left(1+\epsilon+\epsilon^{\prime}\right)$.

$\sum_{i \in B}\left\|E_{i} x\right\| \leq \sum_{i \in B}\left(1+\epsilon+\epsilon^{\prime}\right) G\left(\lambda_{i}\right)=\left(1+\epsilon+\epsilon^{\prime}\right) \sum_{i \in B} G\left(\lambda_{i}\right)$. $G$ é concava, não decrescente e $\sum_{i=1}^{M} \lambda_{i} \leq \lambda$, pela Observação 3.19. então utilizando a desigualdade de Jensen vamos 
mostrar que $\sum_{i \in B} G\left(\lambda_{i}\right) \leq(M-k) G\left(\frac{\lambda}{M-k}\right)$.

$\sum_{i \in B} \frac{1}{M-k} G\left(\lambda_{i}\right)=\frac{1}{M-k} G\left(\lambda_{1}\right)+\frac{1}{M-k} G\left(\lambda_{2}\right)+\ldots+\frac{1}{M-k} G\left(\lambda_{u}\right) \leq G\left(\frac{1}{M-k} \lambda_{1}+\right.$ $\left.\frac{1}{M-k} \lambda_{2}+\ldots+\frac{1}{M-k} \lambda_{u}\right)=G\left(\sum_{i \in B} \frac{1}{M-k} \lambda_{i}\right)=G\left(\frac{1}{M-k} \sum_{i \in B} \lambda_{i}\right) \leq G\left(\frac{\lambda}{M-k}\right)$. Logo $\sum_{i \in B} G\left(\lambda_{i}\right) \leq(M-k) G\left(\frac{\lambda}{M-k}\right)$. Portanto $\sum_{i \in B}\left\|E_{i} x\right\| \leq\left(1+\epsilon+\epsilon^{\prime}\right)(M-k) G\left(\frac{\lambda}{M-k}\right)$.

Consequentemente $\|E x\| \leq g(M)^{-1} \sum_{i=1}^{M}\left\|E_{i} x\right\| \leq M^{-1} G(M)\left(\sum_{i \in B}\left\|E_{i} x\right\|+\sum_{i \in A}\left\|E_{i} x\right\|\right) \leq$ $M^{-1} G(M)\left[\left(1+\epsilon+\epsilon^{\prime}\right)(M-k) G\left(\frac{\lambda}{M-k}\right)+\sum_{i \in A}\left\|E_{i} x\right\|\right]$

$\operatorname{Mas} \sum_{i \in A}\left\|E_{i} x\right\| \leq k\left(\lambda\left(E_{i}\right)(1+\epsilon)+\frac{(1+\epsilon)}{n_{1}}\right) \leq k\left(\frac{(1+\epsilon)(1+\epsilon)}{\epsilon^{\prime} n_{1}}+\frac{(1+\epsilon)}{n_{1}}\right)=$ $\frac{(1+\epsilon)}{n_{1}}\left(\frac{k(1+\epsilon)}{\epsilon^{\prime}}+1\right)=\frac{(1+\epsilon)}{n_{1}}\left(\frac{k(1+\epsilon)+\epsilon^{\prime}}{\epsilon^{\prime}}\right)=\frac{(1+\epsilon)}{\epsilon^{\prime} n_{1}}\left(k(1+\epsilon)+\epsilon^{\prime}\right) \leq \frac{(1+\epsilon)}{\epsilon^{\prime} n_{1}}\left(k(1+\epsilon)+k \epsilon^{\prime}\right)=$ $\frac{k(1+\epsilon)\left(1+\epsilon+\epsilon^{\prime}\right)}{\epsilon^{\prime} n_{1}}$.

Portanto $\|E x\| \leq M^{-1} G(M)\left[\left(1+\epsilon+\epsilon^{\prime}\right)(M-k) G\left(\frac{\lambda}{M-k}\right)+\frac{(1+\epsilon)\left(1+\epsilon+\epsilon^{\prime}\right) k}{\epsilon^{\prime} n_{1}}\right]=\frac{G(M)}{M}(1+\epsilon+$ $\left.\epsilon^{\prime}\right) M\left(1-\frac{k}{M}\right) G\left(\frac{\lambda}{M-k}\right)+\frac{G(M)}{M} \frac{(1+\epsilon)\left(1+\epsilon+\epsilon^{\prime}\right) k}{\epsilon^{\prime} n_{1}} \leq\left(1+\epsilon+\epsilon^{\prime}\right)\left[\left(1-\frac{k}{M}\right) G(M) G\left(\frac{\lambda}{M-k}\right)+\frac{(1+\epsilon) k}{\epsilon^{\prime} n_{1}}\right] \leq$ $\left(1+\epsilon+\epsilon^{\prime}\right)\left[\left(1-\frac{k}{M}\right) G\left(\left(1-\frac{k}{M}\right)^{-1} \lambda\right)+\frac{(1+\epsilon) k}{\epsilon^{\prime} n_{1}}\right]$.

Como $g \geq \sqrt{f}$ temos $g^{\prime}(1) \geq(\sqrt{f(1)})^{\prime}=\frac{1}{2 \sqrt{f(1)}} f^{\prime}(1)=\frac{f^{\prime}(1)}{2}>0$. Pela definição de RIS temos $n_{1} \geq 2(1+\epsilon) M_{f}\left(N / \epsilon^{\prime}\right) / \epsilon^{\prime} f^{\prime}(1), \log \frac{(1+\epsilon) k}{\epsilon^{\prime} n_{1}} \leq \frac{M_{f}\left(N / \epsilon^{\prime}\right)}{M}\left(\frac{(1+\epsilon) k}{\epsilon^{\prime} n_{1}}\right)=$ $\left(\frac{k}{M}\right)\left(\frac{(1+\epsilon) M_{f}\left(N / \epsilon^{\prime}\right)}{\epsilon^{\prime} n_{1}}\right) \leq\left(\frac{k}{M}\right)\left(\frac{(1+\epsilon) M_{f}\left(N / \epsilon^{\prime}\right)}{\epsilon^{\prime}\left(\frac{2(1+\epsilon) M_{f}\left(N / \epsilon^{\prime}\right)}{\epsilon^{\prime} f^{\prime}(1)}\right)}\right)=\left(\frac{k}{M}\right)\left(\frac{f^{\prime}(1)}{2}\right) \leq\left(\frac{k}{M}\right) g^{\prime}(1)$

Colocando $t=\frac{k}{M}$ em $\left(^{*}\right)$ e utilizando a majoração acima para $\frac{(1+\epsilon) k}{\epsilon^{\prime} n_{1}}$ obtemos $\left(1+\epsilon+\epsilon^{\prime}\right)\left[\left(1-\frac{k}{M}\right) G\left(\left(1-\frac{k}{M}\right)^{-1} \lambda\right)+\frac{(1+\epsilon) k}{\epsilon^{\prime} n_{1}}\right] \leq\left(1+\epsilon+\epsilon^{\prime}\right)\left((1-t) G\left(\frac{\lambda}{1-t}\right)+t g^{\prime}(1)\right)$.

Seja $G^{\prime}(1)$ a derivada a direita de $G$ em $1, G^{\prime}(1)=\lim _{x \rightarrow 1^{+}} \frac{G(x)-G(1)}{x-1}=\lim _{x \rightarrow 1^{+}} \frac{G(x)-1}{x-1}$.

Temos que $\lambda \geq 1$ e $0 \leq t<1$, logo como $G$ é concava temos $(1-t) G\left(\frac{\lambda}{1-t}\right)+t G(1) \leq$ $G(\lambda+t)$. Subtraindo $G^{\prime}(1) t$ dos dois lados da desigualdade temos $(1-t) G\left(\frac{\lambda}{1-t}\right)+t G(1)-$ $G^{\prime}(1) t \leq G(\lambda+t)-G^{\prime}(1) t$. Pelo Teorema do valor médio temos $\frac{G(\lambda+t)-G(\lambda)}{t} \leq G^{\prime}(1) \operatorname{logo}$ $(1-t) G\left(\frac{\lambda}{1-t}\right)+t\left(G(1)-G^{\prime}(1)\right) \leq G(\lambda)$ para todo $0 \leq t<1$ e $\lambda \geq 1$. 
Já que $G^{\prime}(x)=\frac{g(x)-x g^{\prime}(x)}{(g(x))^{2}}$ temos que $G(1)-G^{\prime}(1)=1-1+g^{\prime}(1)=g^{\prime}(1)$. Portanto $\left(1+\epsilon+\epsilon^{\prime}\right)\left((1-t) G\left(\frac{\lambda}{1-t}\right)+t g^{\prime}(1)\right) \leq\left(1+\epsilon+\epsilon^{\prime}\right) G(\lambda)$.

Contradizendo nossa suposição sobre o intervalo $E$ e provando o Lema, pois seja $E=\operatorname{ran}(x), \lambda(E)=N \geq 1$ então $\|x\|=\|E x\| \leq\left(1+\epsilon+\epsilon^{\prime}\right) N g(N)^{-1}$.

Teorema 3.22 TS admite um sistema biortogonal assintótico com constante $\theta, \theta \in(0,1 / 2)$.

\section{Demonstração}

Seja $\delta=\frac{\theta}{3-\theta}$, temos que $0<\delta<1$. Sejam $N_{1}<N_{2}<\ldots$ uma sequência de quadrados satisfazendo $f\left(N_{1}\right) / N_{1}<\delta / 2, f\left(N_{1}\right)>8 \delta^{-1}$ e $N_{j}>M_{f}\left(2 N_{j-1}\right)$ para todo $j>1$ e $A_{k}$ o conjunto dos vetores de norma 1 da forma $x=\sum_{i=1}^{N_{k}} x_{i}$ onde $x_{1}=\alpha x_{1}^{\prime}, \ldots, x_{N_{k}}=\alpha x_{N_{k}}^{\prime}$ com $x_{1}^{\prime}, \ldots, x_{N_{k}}^{\prime}$ sendo uma RIS com constante $1+\delta / 2$ e $\alpha \in \mathbb{R}$.

Vamos verificar que o espaço de Thomas Schlumprecht satisfaz uma $f$ - estimativa inferior.

Pela norma do espaço temos $\|x\| \geq \sup \left\{f(N)^{-1} \sum_{i=1}^{N}\left\|E_{i} x\right\|: N \geq 2, E_{1}<\ldots<E_{N}\right\}$. Resta mostrar que $\|E x\| \leq\|x\|, \forall E \subset \mathbb{N}$.

$$
\begin{aligned}
& \text { Vejamos, }\|E x\|=\|E x\|_{\infty} \vee \sup \left\{f(N)^{-1} \sum_{i=1}^{N}\left\|E_{i} E x\right\|: N \geq 2, E_{1}<\ldots<E_{N}\right\} \mathrm{e} \\
& \|x\|=\|x\|_{\infty} \vee \sup \left\{f(N)^{-1} \sum_{i=1}^{N}\left\|E_{i} x\right\|: N \geq 2, E_{1}<\ldots<E_{N}\right\} .
\end{aligned}
$$

Se $\|E x\|=\|E x\|_{\infty}$ temos que $\|E x\|_{\infty} \leq\|x\|_{\infty} \leq\|x\|$.

Se $\|E x\|=\sup \left\{f(N)^{-1} \sum_{i=1}^{N}\left\|E_{i} E x\right\|: N \geq 2, E_{1}<\ldots<E_{N}\right\}$ temos que $\sup \left\{f(N)^{-1} \sum_{i=1}^{N}\left\|E_{\mathrm{i}} E x\right\|: N \geq 2, E_{1}<\ldots<E_{N}\right\} \leq \sup \left\{f(N)^{-1} \sum_{i=1}^{N}\left\|E_{i} x\right\|: N \geq 2, E_{1}<\right.$ $\left.\ldots<E_{N}\right\} \leq\|x\|$. Portanto $\|E x\| \leq\|x\|, \forall E \subset \mathbb{N}$.

$$
\text { Logo }\|x\| \geq \sup \left\{f(N)^{-1} \sum_{i=1}^{N}\left\|E_{i} E x\right\|: N \in \mathbb{N}, E_{1}<\ldots<E_{N}\right\} \text {. }
$$

Como o espaço de Schlumprecht satisfaz uma $f$-estimativa inferior, temos que a constante de multiplicidade $\alpha$ da RIS é menor ou igual a $f\left(N_{k}\right) N_{k}^{-1}$. 
De fato, temos que $\|x\| \geq f(N)^{-1} \sum_{i=1}^{N}\left\|E_{i} x\right\|$ onde $x=\sum_{i=1}^{N_{k}} x_{i}$.

Seja $E_{i}=\operatorname{ran}\left(x_{i}\right)$ então $\|x\| \geq f\left(N_{k}\right)^{-1} \sum_{i=1}^{N_{k}}\left\|x_{i}\right\|=f\left(N_{k}\right)^{-1} \sum_{i=1}^{N_{k}}|\alpha|\left\|x_{i}^{\prime}\right\| \operatorname{logo}\|x\| \geq$ $f\left(N_{k}\right)^{-1} N_{k}|\alpha|$. Consequentemente $|\alpha| \leq f\left(N_{k}\right) N_{k}^{-1}$ já que $\|x\|=1$. Temos também que $\left\|x_{i}\right\|=|\alpha|\left\|x_{i}^{\prime}\right\|=|\alpha| \leq f\left(N_{k}\right) N_{k}^{-1}, i=1, \ldots, N_{k}$.

Seja $A_{k}^{*}$ o conjunto dos funcionais da forma $f\left(N_{k}\right)^{-1} \sum_{i=1}^{N_{k}} x_{i}^{*}$ onde $x_{1}^{*}<\ldots<x_{N_{k}}^{*}$ e $\left\|x_{i}^{*}\right\| \leq 1$ para cada $i, 1 \leq i \leq N_{k}$.

Se $k \leq j-1$ então $N_{j}>M_{f}\left(2 N_{k}\right)$, pois por hipótese $N_{j}>M_{f}\left(2 N_{j-1}\right)$ e $\left(N_{j}\right)_{j}$ é uma sequência estritamente crescente.

Observação: $y^{*} \in A_{j}^{*}$ é uma (M,f)-forma onde $M=N_{j}$. De fato, $y^{*}=\sum_{i=1}^{M} f(M)^{-1} x_{i}^{*}$, onde $x_{1}^{*}<\ldots<x_{M}^{*},\left\|x_{i}^{*}\right\| \leq 1$ para qualquer $i, 1 \leq i \leq M$. $\left\|f(M)^{-1} x_{i}^{*}\right\|=f(M)^{-1}\left\|x_{i}^{*}\right\| \leq f(M)^{-1}, \forall i$. Seja $z \in T S$ com $\|z\| \leq 1$. Vamos calcular $\left|y^{*}(z)\right|$.

$\left|y^{*}(z)\right|=\left|\sum_{i=1}^{M} f(M)^{-1} x_{i}^{*}(z)\right| \leq f(M)^{-1} \sum_{i=1}^{M}\left|x_{i}^{*}(z)\right|$. Consideremos $E_{i}=\operatorname{ran}\left(x_{i}^{*}\right)$ então

$f(M)^{-1} \sum_{i=1}^{M}\left|x_{i}^{*}(z)\right|=f(M)^{-1} \sum_{i=1}^{M}\left|\left(E_{i} x_{i}^{*}\right)(z)\right|=f(M)^{-1} \sum_{i=1}^{M}\left|x_{i}^{*}\left(E_{i} z\right)\right| \leq$

$\leq f(M)^{-1} \sum_{i=1}^{M}\left\|x_{i}^{*}\right\|\left\|E_{i} z\right\| \leq f(M)^{-1} \sum_{i=1}^{M}\left\|E_{i} z\right\| \leq\|z\|$. Portanto $\left\|y^{*}\right\| \leq 1$. Logo $y^{*} \in A_{j}^{*}$ é uma (M,f)-forma onde $M=N_{j}$.

Passaremos a verificar que se $x \in A_{k}$ e $y^{*} \in A_{j}^{*}, j \neq k$, então $\left|y^{*}(x)\right|<\delta$.

Para $j>k$ vamos aplicar o Lema $3.16 \operatorname{com} \epsilon=\frac{1}{2}, M=N_{j}, f=g, y^{*} \in A_{j}^{*}$, que já vimos que é uma ( $\mathrm{M}, \mathrm{f})$-forma, e sabendo que $1+\delta / 2<1+1 / 2=1+\epsilon$.

No Lema $3.16 x=\sum_{i=1}^{N} x_{i}$ onde $x_{1}, \ldots, x_{N}$ é uma RIS. No nosso contexto $x=\sum_{i=1}^{N_{k}} x_{i}$ onde $x_{1}, \ldots, x_{N_{k}}$ é multiplo de uma RIS então temos $\left|y^{*}(x)\right|=|\alpha|\left|y^{*}\left(\frac{x}{\alpha}\right)\right| \leq \frac{f\left(N_{k}\right)}{N_{k}}\left(1+\epsilon+\epsilon^{\prime}\right)=$ $2 \frac{f\left(N_{k}\right)}{N_{k}}$. Portanto $\left|y^{*}(x)\right| \leq 2 \frac{f\left(N_{k}\right)}{N_{k}}$.

Como $\frac{x}{f(x)}$ é não decrescente temos que $\frac{f\left(x_{2}\right)}{x_{2}}<\frac{f\left(x_{1}\right)}{x_{1}}$ para $x_{1}<x_{2}$. 
Por construção $N_{1}<N_{2}<\ldots . \operatorname{logo} \ldots<\frac{f\left(N_{3}\right)}{N_{3}}<\frac{f\left(N_{2}\right)}{N_{2}}<\frac{f\left(N_{1}\right)}{N_{1}}<\frac{\delta}{2}$, ou seja $\frac{f\left(N_{k}\right)}{N_{k}}<\frac{\delta}{2}$.

Por este resultado temos que $\left|y^{*}(x)\right| \leq 2 \frac{f\left(N_{k}\right)}{N_{k}}<2 \frac{\delta}{2}=\delta$. Portanto $\left|y^{*}(x)\right|<\delta$ para qualquer $y^{*} \in A_{j}^{*}$ e $x \in A_{k}, j>k$.

Se $j<k$ vamos aplicar o Lema 3.21 para $y=\frac{x}{\alpha}$, que é a soma da RIS $x_{1}^{\prime}, \ldots, x_{N_{k}}^{\prime}$, com $\epsilon=\frac{1}{2}$ e $f=g$, mas para isto vamos primeiro verificar que $\|E y\| \leq \sup \left\{\left|x^{*}(E y)\right|\right.$ : $M \geq 2, x^{*}$ é uma (M,f)-forma $\}$ para qualquer intervalo $E$ tal que $\lambda(E) \geq 1$.

Pela norma do espaço temos que $\|E y\|=\|E y\|_{\infty}$ ou $\|E y\|=\sup \left\{f(N)^{-1} \sum_{i=1}^{N}\left\|E_{i}(E y)\right\|\right.$, $N \geq 2\}$. Verificaremos que para $\lambda(E) \geq 1,\|E y\|=\sup \left\{f(N)^{-1} \sum_{i=1}^{N}\left\|E_{i}(E y)\right\|, N \geq 2\right\}$.

Seja $E$ um intervalo tal que $\lambda(E) \geq 1$. Temos que $y=\sum_{i=1}^{N_{k}} x_{i}^{\prime}$ onde $x_{1}^{\prime}, \ldots, x_{N_{k}}^{\prime}$ é uma RIS, logo cada $x_{i}^{\prime}$ é um $l_{1+}^{n_{i}}$ - average.

Pela definição de $l_{1+}^{n_{1}}$ - average temos que $x_{i}^{\prime}=x_{i 1}+x_{i 2}+\ldots+x_{i n_{i}}$ onde $\left\|x_{i j}\right\| \leq \frac{1+\epsilon}{n_{i}} \leq$ $\frac{1+\epsilon}{n_{1}}$. Pela definição de RIS sabemos que $n_{1} \geq \frac{2(1+\epsilon) M_{f}\left(N_{k} / \epsilon^{\prime}\right)}{\epsilon^{\prime} f^{\prime}(1)}$. Seja $M_{f}\left(N_{k} / \epsilon^{\prime}\right)=x_{0}$ então $f\left(x_{0}\right)=36\left(N_{k} / \epsilon^{\prime}\right)^{2} \geq 36\left(N_{k}\right)^{2}>N_{k}$, já que $\left(\epsilon^{\prime}\right)^{2} \leq 1$. Portanto $\log _{2}\left(x_{0}+1\right)>N_{k}$, logo $x_{0}>2^{N_{k}}-1$.

$f^{\prime}(1) \leq 1$ e $\epsilon^{\prime} \leq 1$ consequentemente $n_{1} \geq \frac{2(1+\epsilon) x_{0}}{1.1}>2(1+\epsilon)\left(2^{N_{k}}-1\right)$. Com isto temos que $\frac{1+\epsilon}{n_{1}}<\frac{1+\epsilon}{2(1+\epsilon)\left(2^{N_{k}}-1\right)}=\frac{1}{2\left(2^{N_{k}}-1\right)}<\frac{1}{4}$, pois $\frac{1}{2^{N_{k}-1}}<\frac{1}{2} \Longleftrightarrow 2<2^{N_{k}}-1$, o que é verdadeiro.

Portanto $\left\|x_{i j}\right\|<\frac{1}{4}$ e como $\left\|x_{i j}\right\|_{\infty} \leq\left\|x_{i j}\right\|$ segue que $\|E y\|_{\infty} \leq\|y\|_{\infty}<\frac{1}{4}$.

Mas já verificamos no Lema 3.20 que para intervalos com $\lambda(E) \geq 1$ e $0<\epsilon \leq \frac{1}{2}$ temos que $\|E y\| \geq \frac{1}{4}$.

Portanto $\|E y\|$ não é $\|E y\|_{\infty}$, ou seja, para $\lambda(E) \geq 1$ e $0<\epsilon \leq \frac{1}{2}$ não ocorre a norma do supremo. Sendo assim $\|E y\|=\sup \left\{f(N)^{-1} \sum_{i=1}^{N}\left\|E_{i}(E y)\right\|, N \geq 2\right\}$ para $\lambda(E) \geq 1$.

Então temos $\|E y\|-\epsilon<\frac{1}{f(n)} \sum_{i=1}^{n}\left\|E_{i} E y\right\|$. Consideremos $z_{i}^{*}$ o funcional suporte de $E_{i}(E y), \forall i, 1 \leq i \leq n$ e $x^{*}=f(n)^{-1} \sum_{i=1}^{n} z_{i}^{*}$. A definição de $z_{i}^{*}$ é análoga a feita na demonstração do Corolário 3.17 e como vimos nesta demonstração $x^{*}$ é uma $(M, f)$-forma, 
$M \geq 2$.

Logo $x^{*}(E y)=f(n)^{-1} \sum_{i=1}^{n} z_{i}^{*}(E y)=f(n)^{-1} \sum_{i=1}^{n}\left\|E_{i} E y\right\|>\|E y\|-\epsilon$. Fazendo $\epsilon \rightarrow 0$ temos $\|E y\| \leq x^{*}(E y)$.

Portanto $\|E y\| \leq \sup \left\{\left|x^{*}(E y)\right|: M \geq 2, x^{*}\right.$ é uma (M,f)-forma $\}$ para qualquer intervalo $E$ tal que $\lambda(E) \geq 1$.

Então aplicando o Lema $3.21 \operatorname{com} \epsilon=\frac{1}{2}$ e $f=g$ temos $\left\|\sum_{i \in A} x_{i}\right\|=|\alpha|\left\|\sum_{i \in A} \frac{x_{i}}{\alpha}\right\| \leq$ $\frac{2 f\left(N_{k}\right)}{N_{k}} \frac{|A|}{f(|A|)}=\frac{2|A| f\left(N_{k}\right)}{N_{k} f(|A|)}$ para qualquer subconjunto $A$ de $\left\{1,2, \ldots, N_{k}\right\}$.

Se $|A| \geq \sqrt{N_{k}}$ então $f(|A|) \geq f\left(\sqrt{N_{k}}\right)$ logo $\frac{1}{f(|A|)} \leq \frac{1}{f\left(\sqrt{N_{k}}\right)}$. Com isto segue que $\frac{2 f\left(N_{k}\right)|A|}{N_{k} f(|A|)} \leq \frac{2 f\left(N_{k}\right)|A|}{N_{k} f\left(\sqrt{N_{k}}\right)}$.

Vamos mostrar que $\frac{f\left(N_{k}\right)}{f\left(\sqrt{N_{k}}\right)} \leq 2$, ou seja, $\frac{\log _{2}\left(N_{k}+1\right)}{\log _{2}\left(\sqrt{N_{k}}+1\right)} \leq 2$.

$\frac{\log _{2}\left(N_{k}+1\right)}{\log _{2}\left(\sqrt{\left.N_{k}+1\right)}\right.} \leq 2 \Longleftrightarrow \log _{2}\left(N_{k}+1\right) \leq 2 \log _{2}\left(\sqrt{N_{k}}+1\right) \Longleftrightarrow \log _{2}\left(N_{k}+1\right) \leq \log _{2}\left(\sqrt{N_{k}}+1\right)^{2} \Longleftrightarrow$ $N_{k}+1 \leq\left(\sqrt{N_{k}}+1\right)^{2}$ o que é verdadeiro. Sendo assim $\frac{2 f\left(N_{k}\right)|A|}{N_{k} f(|A|)} \leq \frac{4|A|}{N_{k}}$.

Dividindo $x$ em pedaços sucessivos de tamanho $\sqrt{N_{k}}$, podemos verificar que $x$ é um $l_{1+}^{\sqrt{N_{k}}}$-average com constante 4 .

De fato, $x=\sum_{i=1}^{N_{k}} x_{i}$ considerando $y_{1}=\sum_{i=1}^{\sqrt{N_{k}}} x_{i}, y_{2}=\sum_{i=\sqrt{N_{k}}+1}^{\sqrt[2]{N_{k}}} x_{i}, \ldots, y \sqrt{N_{k}}=\sum_{i=\left(\sqrt{N_{k}}-1\right) \sqrt{N_{k}}+1}^{\sqrt{N_{k}} \sqrt{N_{k}}} x_{i}$ temos que $x=\sum_{i=1}^{\sqrt{N_{k}}} y_{i},\|x\|=1$ e $\left\|y_{i}\right\| \leq \frac{4 \sqrt{N_{k}}}{N_{k}}=\frac{4 \sqrt{N_{k} \sqrt{N_{k}}}}{N_{k} \sqrt{N_{k}}}=\frac{4 N_{k}}{N_{k} \sqrt{N_{k}}}=4\left(\sqrt{N_{k}}\right)^{-1}$.

Portanto pela definição de $l_{1+}^{n}$-average temos que $x=\sum_{i=1}^{\sqrt{N_{k}}} y_{i}$ é um $l_{1+}^{\sqrt{N_{k}}}$-average com constante 4. Vamos usar o Lema 3.13 para calcular $\left|y^{*}(x)\right|$. $\left|y^{*}(x)\right|=\left|f\left(N_{j}\right)^{-1} \sum_{i=1}^{N_{j}} x_{i}^{*}(x)\right| \leq f\left(N_{j}\right)^{-1} \sum_{i=1}^{N_{j}}\left|x_{i}^{*}(x)\right|$. Considerando $E_{i}=\operatorname{ran}\left(x_{i}^{*}\right)$ temos $f\left(N_{j}\right)^{-1} \sum_{i=1}^{N_{j}}\left|x_{i}^{*}(x)\right|=f\left(N_{j}\right)^{-1} \sum_{i=1}^{N_{j}}\left|\left(E_{i} x_{i}^{*}\right)(x)\right|=f\left(N_{j}\right)^{-1} \sum_{i=1}^{N_{j}}\left|x_{i}^{*}\left(E_{i} x\right)\right| \leq$ $\leq f\left(N_{j}\right)^{-1} \sum_{i=1}^{N_{j}}\left\|x_{i}^{*}\right\|\left\|E_{i} x\right\| \leq f\left(N_{j}\right)^{-1} \sum_{i=1}^{N_{j}}\left\|E_{i} x\right\| \leq f\left(N_{j}\right)^{-1} 4\left(1+\frac{2 N_{j}}{\sqrt{N_{k}}}\right)$.

Vamos provar que $\frac{2 N_{j}}{\sqrt{N_{k}}} \leq 1$ quando $j<k$. Para isto basta mostrar que $\frac{2 N_{j-1}}{\sqrt{N_{j}}} \leq 1$ já 
que $j<k$.

Por hipótese sabemos que $N_{j}>M_{f}\left(2 N_{j-1}\right) \operatorname{logo} f\left(N_{j}\right)>36\left(2 N_{j-1}\right)^{2}=144 N_{j-1}^{2}$, ou seja, $\log _{2}\left(N_{j}+1\right)>144 N_{j-1}^{2}$. Mas $4 N_{j} \geq N_{j}+1 \geq \log _{2}\left(N_{j}+1\right)$ portanto $4 N_{j}>144 N_{j-1}^{2}$, isto é, $2 \sqrt{N_{j}}>12 N_{j-1}$. Em particular, $2 \sqrt{N_{j}}>4 N_{j-1}$ e portanto $\sqrt{N_{j}}>2 N_{j-1}$. E com isto temos o resultado $\frac{2 N_{j-1}}{\sqrt{N,}}<1$.

$\operatorname{Assim}\left|y^{*}(x)\right| \leq 8 f\left(N_{j}\right)^{-1}<8 \frac{\delta}{8}=\delta$, pois $\frac{8}{\delta}<f\left(N_{1}\right)<f\left(N_{2}\right)<\ldots$

Portanto $\left|y^{*}(x)\right|<\delta$ para $x \in A_{k}$ e $y^{*} \in A_{j}^{*}, j<k$.

Verificaremos agora que se $x \in A_{k}$ e $y^{*} \in A_{k}^{*}$ então $y^{*}(x)>1-\delta$.

Novamente, aplicando o Lema 3.21 com $f=g$, sabendo que $0<\frac{\delta}{2}<\frac{1}{2}$, $\frac{x}{\alpha}$ é uma RIS com constante $1+\frac{\delta}{2}$ e que $\left\|E \frac{x}{\alpha}\right\| \leq \sup \left\{\left|x^{*}\left(E \frac{x}{\alpha}\right)\right|: M \geq 2, x^{*}\right.$ é uma (M,f)-forma \} , para $\lambda(E) \geq 1$, temos $\left\|\frac{x}{\alpha}\right\| \leq\left(1+\frac{\delta}{2}+\frac{\delta}{2}\right) N_{k} f\left(N_{k}\right)^{-1}=(1+\delta) N_{k} f\left(N_{k}\right)^{-1}$. Portanto $\|x\| \leq(1+\delta) N_{k} f\left(N_{k}\right)^{-1}|\alpha|=(1+\delta) N_{k} f\left(N_{k}\right)^{-1}\left\|x_{i}\right\|$, para todo $i$.

Seja $E_{i}=\operatorname{ran}\left(x_{i}\right), i=1, \ldots, N_{k}$ e consideremos $z_{i}^{*}$ o funcional suporte de $E_{i} x$, $i=1, \ldots, N_{k}$. A definição do funcional suporte é análoga a feita na demonstração do Corolário 3.17. Seja $x^{*}=f\left(N_{k}\right)^{-1} \sum_{i=1}^{N_{k}} z_{i}^{*}, x^{*}$ é um elemento de $A_{k}^{*}$. De fato, já sabemos da demonstração do Corolário 3.17 que $\left\|z_{i}^{*}\right\| \leq 1$ para todo $i$ e que $z_{1}^{*}<z_{2}^{*}<\ldots<z_{N_{k}}^{*}$. Portanto $x^{*} \in A_{k}^{*}$.

$x^{*}(x)=f\left(N_{k}\right)^{-1} \sum_{i=1}^{N_{k}} z_{i}^{*}(x)=f\left(N_{k}\right)^{-1} \sum_{i=1}^{N_{k}}\left\|x_{i}\right\| \geq \frac{N_{k}\|x\| f\left(N_{k}\right)}{f\left(N_{k}\right)(1+\delta) N_{k}}=\frac{\|x\|}{(1+\delta)}=\frac{1}{(1+\delta)}=$ $(1+\delta)^{-1}$. Temos que $(1+\delta)^{-1}>1-\delta$, pois $(1+\delta)^{-1}>1-\delta \Longleftrightarrow(1+\delta)(1-\delta)<$ $1 \Longleftrightarrow-\delta^{2}<0$ o que é verdade. Logo $x^{*}(x)>(1+\delta)^{-1}>1-\delta$, para $x \in A_{k}$ e $x^{*} \in A_{k}^{*}$.

$\operatorname{Sejam} B_{k}=\left\{\frac{x}{\|x\|}: \exists a \in A_{k},\|x-a\|<\delta\right\}$ e $B_{k}^{*}=A_{k}^{*}$.

Pela definição de $B_{k}$ temos que $A_{k} \subset B_{k}$.

Afirmação: $\left(B_{k}, B_{k}^{*}\right)$ é um sistema biortogonal assintótico com constante $\theta$ para TS.

De fato,

1) $B_{k}$ é assintótico para todo $k$.

Seja $Y$ um subespaço de dimensão infinita de $T S$, logo segue da Proposição 2.4, [15], que existe uma sequência $\left(z_{i}\right)_{i}$ normalizada tal que $\left\|z_{i}-z_{j}\right\| \geq 1$ para $j<i$. 
Como $l_{1}(\mathbb{N}) \nLeftarrow \bar{Y} \subset T S$ (pág 85, [22]), temos pelo Teorema 0.16 que existe uma subsequência $\left(z_{i_{n}}\right)_{n}$ de $\left(z_{i}\right)_{i}$ tal que $\left(z_{i_{n}}\right)_{n}$ é fraca de Cauchy.

Seja $y_{n}=\frac{z_{i_{n}}-z_{i_{n+1}}}{\left\|z_{i_{n}}-z_{i_{n+1}}\right\|}$, então claramente $\left(y_{n}\right)_{n}$ é fraca nula.

Consideremos $N_{k}$ e fixemos $n_{1} \geq 2\left(1+\frac{\delta}{2}\right) M_{f}\left(\frac{N_{k}}{\delta^{\prime}}\right) / \delta^{\prime} f^{\prime}(1)$ e $\frac{\delta}{2 n_{1} N_{k}}<\frac{1}{8}$. Tomemos $q$ um inteiro tal que $q \log \left(1+\frac{\delta}{2}\right)>\log f\left(n_{1}^{q}\right)$ e coloquemos $M_{1}=n_{1}^{q}$.

Seja $y_{11}=y_{1}$, como $c_{00}$ é denso em TS existe $c_{11} \in c_{00}$ tal que $\left\|y_{11}-c_{11}\right\|<\frac{\delta}{2 n_{1} N_{k}}$.

Logo $\left\|y_{11}\right\| \leq\left\|y_{11}-c_{11}\right\|+\left\|c_{11}\right\|$, ou seja, $1 \leq \frac{1}{8}+\left\|c_{11}\right\|$. Portanto $t_{11}=\left\|c_{11}\right\| \geq \frac{7}{8} \geq \frac{1}{2}$.

Seja $m_{1}$ tal que $\operatorname{supp}\left(c_{11}\right) \subset\left[1, m_{1}\right]$. Como $y_{n} \rightarrow 0$ fracamente, existe $y_{12} \in\left\{y_{n}\right\}_{n}$ tal que a j-essima coordenada de $y_{12}$ fica pequena $\forall j, 1 \leq j \leq m_{1}$, então podemos supor que $\left\|P_{m_{1}}\left(y_{12}\right)\right\|<\frac{\delta}{6 n_{1} N_{k}}$, onde $P_{m_{1}}$ é a projeção de $y_{12}$ sobre o intervalo [1, $\left.m_{1}\right]$.

Novamente existe $d_{12} \in c_{00}$ tal que $\left\|y_{12}-d_{12}\right\|<\frac{\delta}{6 n_{1} N_{k}}$.

Seja $c_{12}$ a projeção de $d_{12}$ no intervalo $\left[m_{1}+1,+\infty\right)$.

Podemos notar que $\left\|P_{m_{1}}\left(d_{12}\right)-P_{m_{1}}\left(y_{12}\right)\right\| \leq\left\|d_{12}-y_{12}\right\|<\frac{\delta}{6 n_{1} N_{k}}$. Logo $\left\|P_{m_{1}}\left(d_{12}\right)\right\| \leq\left\|P_{m_{1}}\left(d_{12}\right)-P_{m_{1}}\left(y_{12}\right)\right\|+\left\|P_{m_{1}}\left(y_{12}\right)\right\|<\frac{\delta}{6 n_{1} N_{k}}+\frac{\delta}{6 n_{1} N_{k}}=\frac{\delta}{3 n_{1} N_{k}} \leq \frac{1}{12}$.

Como $c_{12}=d_{12}-P_{m_{1}}\left(d_{12}\right)$, temos que $\left\|d_{12}\right\| \leq\left\|c_{12}\right\|+\left\|P_{m_{1}}\left(d_{12}\right)\right\|$, análogo ao que fizemos para $\left\|c_{11}\right\|$ temos que $\left\|d_{12}\right\| \geq \frac{23}{24}$. Portanto $\left\|c_{12}\right\| \geq \frac{23}{24}-\frac{1}{12} \geq \frac{1}{2}$. Coloquemos $t_{12}=\left\|c_{12}\right\|$.

Continuando este processo encontraremos $c_{11}, c_{12}, \ldots, c_{1 M_{1}} \in c_{00}, c_{11}<c_{12}<\ldots<c_{1 M_{1}}$ e $y_{11}, y_{12}, \ldots, y_{1 M_{1}} \in Y$ com $t_{1 i}=\left\|c_{1 i}\right\| \geq \frac{1}{2}, \forall i, 1 \leq i \leq M_{1}$ satisfazendo $\left\|y_{1 i}-c_{1 i}\right\|<$ $\frac{\delta}{2 n_{1} N_{k}}, \forall i, 1 \leq i \leq M_{1}$.

Pela observação 3.12 , possivelmente renumerando os índices, nós podemos supor que $\left(\frac{c_{11}}{t_{11}}+\ldots+\frac{c_{1 n_{1}}}{t_{1 n_{1}}}\right)$ é um $l_{1+}^{n_{1}}$ - average com constante $1+\frac{\delta}{2}$.

De maneira análoga é possível construir $N_{k} l_{1+}^{n_{l}}$ - average $\left(\frac{c_{11}}{t_{l 1}}+\ldots+\frac{c_{l_{l}}}{t_{l n_{l}}}\right)$ satisfazendo a definição de RIS.

Sejam $b=\frac{c_{11}}{t_{11}}+\ldots+\frac{c_{1 n_{1}}}{t_{1 n_{1}}}+\ldots+\frac{c_{N_{k} 1}}{t_{N_{k} 1}}+\ldots+\frac{c_{N_{k} n_{N_{k}}}}{t_{N_{k^{n}} N_{k}}}$ e $s=\|b\| \geq\left\|\frac{c_{11}}{t_{11}}+\ldots+\frac{c_{1 n_{1}}}{t_{1 n_{1}}}\right\|=1$.

Logo $a=\frac{b}{s} \in A_{k}$. Tomemos $x=\frac{1}{s}\left(\frac{y_{11}}{t_{11}}+\ldots+\frac{y_{1 n_{1}}}{t_{1 n_{1}}}+\ldots+\frac{y_{N_{k} 1}}{t_{N_{k} 1}}+\ldots+\frac{y_{N_{k} n_{N_{k}}}}{t_{N_{k} n_{N_{k}}}}\right) \in Y$. 
Logo $\|x-a\| \leq \frac{1}{s}\left(\left\|\frac{y_{11}}{t_{11}}-\frac{c_{11}}{t_{11}}\right\|+\ldots+\left\|\frac{y_{N_{k} n_{N_{k}}}}{t_{N_{k} n_{N_{k}}}}-\frac{c_{N_{k} n_{N_{k}}}}{t_{N_{k} n_{N_{k}}}}\right\|\right)<\frac{1}{s} 2\left[n_{1} \frac{\delta}{2 n_{1} N_{k}}+n_{2} \frac{\delta}{2 n_{2} N_{k}}+\ldots\right.$ $\left.+n_{N_{k}} \frac{\delta}{2 n_{N_{k}} N_{k}}\right]<\delta$. Portanto $\frac{x}{\|x\|}$ pertence a $B_{k}$ e pertence a $S(Y)$, ou seja $B_{k} \cap S(Y) \neq \emptyset$, consequentemente $B_{k}$ é assintótico.

2) Seja $b=\frac{x}{\|x\|} \in B_{k}$, logo existe $a \in A_{k}$ tal que $\|x-a\|<\delta$, em particular, $\|x\| \leq 1+\delta$. Portanto existe $a^{*} \in A_{k}^{*}$ tal que $a^{*}(a)>1-\delta$.

De $\left|a^{*}(x)-a^{*}(a)\right|<\delta$, segue que $1-2 \delta<a^{*}(a)-\delta<a^{*}(x)$ e portanto $1-\theta=$ $\frac{1-2 \delta}{1+\delta} \leq \frac{1-2 \delta}{\|x\|} \leq a^{*}(b)$. Portanto $a^{*}(b) \geq 1-\theta$.

3) Sejam $n \neq m, b=\frac{x}{\|x\|} \in B_{n}$ e $a^{*} \in A_{m}^{*}$.

Existe $a \in A_{n}$ tal que $\|x-a\|<\delta$ e $\left|a^{*}(a)\right|<\delta$, em particular, $\|x\|>1-\delta$.

Consequentemente $\left|a^{*}(x)\right| \leq\left|a^{*}(x)-a^{*}(a)\right|+\left|a^{*}(a)\right|<2 \delta$, logo $\left|a^{*}\left(\frac{x}{\|x\|}\right)\right| \leq \frac{2 \delta}{1-\delta}<\theta$.

Por 1), 2) e 3) temos que ( $\left.B_{k}, B_{k}^{*}\right)$ é um sistema biortogonal assintótico com constante $\theta$ para $T S$.

Corolário 3.23 Fixado $C \in \mathbb{R}, C>1$ o espaço TS pode ser renormado de forma a não conter nenhuma sequência básica $C$-incondicional.

\section{Demonstração}

Segue do Teorema acima e do Teorema 3.3. 


\section{Capítulo 4}

\section{O espaço de Gowers e Maurey}

Neste capítulo finalmente construiremos o espaço de Gowers e Maurey. Como já foi dito este espaço foi o primeiro exemplo de espaço de Banach hereditariamente indecomponível.

A sua construção é bem parecida com a construção do espaço de Thomas Schlumprecht feita no capítulo anterior.

Inicialmente fixaremos um, particular, subconjunto infinito $J$ dos naturais e no Lema 4.2 estudaremos todas as propriedades de $J$ usadas no decorrer do capítulo.

Em seguida fixada uma norma qualquer $\|$.$\| em c_{00}$ e dividindo $J$ em dois, particulares, subconjuntos infinitos disjuntos definiremos o que chamaremos funcionais especiais em $\left(c_{00},\|\cdot\|\right)$.

E assim, estaremos em condições de partindo de $c_{00}$ com a norma do supremo definir indutivamente o espaço de Gowers e Maurey.

Finalmente, com o auxilio de quatro Lemas provaremos que esse espaço é hereditariamente indecomponivel, e a partir desta propriedade verificaremos que ele também não possue nenhuma sequência básica incondicional.

A teoria apresentada neste capítulo pode ser encontrada em [11].

Como no capítulo anterior fixemos $f(x)=\log _{2}(x+1)$. 
Definição 4.1 Seja $J \subset \mathbb{N}$ o conjunto denotado por $\left\{j_{1}, j_{2}, \ldots\right\}$ onde, $j_{1}=10^{10^{10^{400}}}$, $j_{2}=10^{10^{10^{4}\left(\jmath_{1}\right)^{2}}}, \ldots, j_{n+1}=10^{10^{10^{4(j n)^{2}}}}$.

Lema 4.2 Para os elementos do conjunto J temos:

1) $\left(j_{n}\right)^{1 / 40} \in \mathbb{N}, \forall n$.

2) $j_{m}>j_{n} \Rightarrow \ln \ln \ln j_{m} \geq 4\left(j_{n}\right)^{2}$.

3) $f\left(j_{1}\right)>256$.

4) $j_{n} \geq n+2, \forall n$. Em particular, $j_{n-1} \geq n+1$ e portanto $\left(j_{n-1}\right)^{2} \geq n^{2}+1$.

5) $j_{n} \geq 10^{\left(10^{10}\right)\left(n^{2}+1\right)}, \forall n$.

6) $\left(j_{2 k}\right)^{1 / 40} \geq \frac{40 M_{f}(10 k)}{f^{\prime}(1)}, \forall k$.

7) $j_{2 k} \geq 16 k^{4}, \forall k$.

\section{Demonstração}

1) Vamos fazer a prova por indução.

$n=1,\left(j_{1}\right)^{1 / 40}=\left(10^{10^{10^{400}}}\right)^{1 / 40}=10^{10^{10^{10}}}$ que é inteiro.

$n=2,\left(j_{2}\right)^{1 / 40}=\left(10^{10^{\left.10^{4\left(\jmath_{1}\right.}\right)^{2}}}\right)^{1 / 40}=10^{10^{10^{\left(\jmath_{1}\right)^{2} / 10}}}$ que é inteiro, pois $\left(j_{1}\right)^{2}$ é divisivel por 10.

Suponha que $j_{n}$ é inteiro, vamos provar que $j_{n+1}$ é inteiro.

$\left(j_{n+1}\right)^{1 / 40}=\left(10^{10^{10^{4}\left(J_{n}\right)^{2}}}\right)^{1 / 40}=10^{10^{\left.10^{(j n}\right)^{2} / 10}}$ que é inteiro, pois $\left(j_{n}\right)^{2}$ é divisivel por 10.

Portanto $\left(j_{n}\right)^{1 / 40} \in \mathbb{N}, \forall n \in \mathbb{N}$.

2) De fato, $\ln \ln \ln j_{m} \geq \log \log \log j_{m} \geq \log \log \log j_{n+1}=4\left(j_{n}\right)^{2}$.

3) De fato, $f\left(j_{1}\right)=\log _{2}\left(1+10^{10^{10^{400}}}\right) \geq \log _{2}\left(10^{10^{10^{400}}}\right) \geq 10^{10^{400}}>256$.

4) Vamos fazer a prova por indução.

$n=1, j_{1} \geq 3$. Suponha que $i+2 \leq j_{i}, \forall i \leq n$. Vamos provar que $n+3 \leq j_{n+1}$.

Ora $j_{n+1}=10^{10^{\left.10^{4(j n}\right)^{2}}} \geq 4\left(j_{n}\right)^{2} \geq j_{n} j_{n} \geq(n+2)(n+2)=(n+2)^{2} \geq(n+2)+1=n+3$.

Portanto $j_{n} \geq n+2, \forall n$.

5) De fato,

$j_{n} \geq 10^{\left(10^{10}\right)\left(n^{2}+1\right)} \Longleftrightarrow \log j_{n} \geq \log 10^{\left(10^{10}\right)\left(n^{2}+1\right)} \Longleftrightarrow 10^{10^{4\left(j_{n-1}\right)^{2}}} \geq\left(10^{10}\right)\left(n^{2}+1\right) \Longleftrightarrow$ $\log 10^{10^{4\left(J_{n-1}\right)^{2}}} \geq \log \left(10^{10}\right)\left(n^{2}+1\right) \Longleftrightarrow 10^{4\left(j_{n-1}\right)^{2}} \geq 10 \log 10+\log \left(n^{2}+1\right) \Longleftrightarrow 10^{4\left(j_{n-1}\right)^{2}} \geq$ $10+\log \left(n^{2}+1\right)$. 
Mas $10^{4\left(j_{n-1}\right)^{2}}=\left(10^{4}\right)^{\left(j_{n-1}\right)^{2}} \geq(10+10)^{n^{2}+1} \geq 10^{n^{2}+1}+10^{n^{2}+1} \geq 10+\log \left(n^{2}+1\right)$. pois $\log x \leq 10^{x}$ e $(a+a)^{n} \geq a^{n}+a^{n}, \forall a \in \mathbb{R}_{+}$. Portanto $j_{n} \geq 10^{\left(10^{10}\right)\left(n^{2}+1\right)}, \forall n$.

6) $f^{\prime}(1)=\frac{1}{2} \log _{2} e, \log 0 \frac{1}{2} \leq f^{\prime}(1) \leq 1$.

Como $f^{\prime}(1) \geq \frac{1}{2}$, basta provarmos que $\left(j_{2 k}\right)^{1 / 40} \geq 80 M_{f}(10 k), \forall k$.

Seja $x_{0}$ tal que $M_{f}(10 k)=x_{0}, \log 0 f\left(x_{0}\right)=36(10 k)^{2}$, ou seja, $\log _{2}\left(x_{0}+1\right)=36(10 k)^{2}$ e portanto $x_{0}+1=2^{36 \cdot 5^{2} \cdot(2 k)^{2}}$.

Como $\left(80 x_{0}\right)^{40} \leq\left(80\left(x_{0}+1\right)\right)^{40}$ temos que provar que $\left(80.2^{36.5^{2} \cdot(2 k)^{2}}\right)^{40} \leq j_{2 k}$, isto é. $80^{40} 2^{36.5^{2} \cdot 40 .(2 k)^{2}} \leq j_{2 k}$.

Mas $(80)^{40} 2^{36.5^{2} \cdot 40 \cdot(2 k)^{2}} \leq\left(10^{10^{10}}\right) 10^{40^{4}(2 k)^{2}} \leq\left(10^{10^{10}}\right) 10^{\left(10^{10}\right)(2 k)^{2}} \leq 10^{\left(10^{10}\right)\left((2 k)^{2}+1\right)} \leq$ $j_{2 k}$ pelo ítem j. Portanto $\left(j_{2 k}\right)^{1 / 40} \geq \frac{40 M_{f}(10 k)}{f^{\prime}(1)} \forall k$.

7) Vamos fazer a prova por indução.

Para $k=1.16 \leq j_{2}$. Suponha que $16 k^{4} \leq j_{2 k}$ para $k \leq n$.

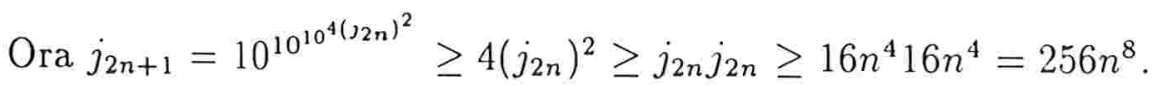

$256 n^{8} \geq 16(n+1)^{4} \Longleftrightarrow 16 n^{8} \geq(n+1)^{4} \Longleftrightarrow 4 n^{4} \geq(n+1)^{2} \Longleftrightarrow 4\left(n^{2} n^{2}\right) \geq(n+1)(n+1)$ o que é verdade pois $n^{2} \geq n+1$.

Definição 4.3 Sejam $K$ e $L$ os subconjuntos de $J$ dados por $K=\left\{j_{1}, j_{3}, j_{5} \ldots\right\}$ e $L=$ $\left\{j_{2}, j_{4}, j_{6}, \ldots\right\}, Q$ o subconjunto de $c_{00}$ consistindo de todas as sequências de coordenadas racionais no intervalo $[-1,1]$ e $\sigma$ uma função injetora do conjunto das sequências finitas de elementos sucessivos de $Q$ com imagem no conjunto $L$ com a propriedade de que se $z_{1}, \ldots, z_{s}$ é uma qualquer dessas sequências sucessivas, $S=\sigma\left(z_{1}, \ldots, z_{s}\right)$ e $z=\sum_{i=1}^{s} z_{i}$, então $\frac{1}{20} f\left(S^{1 / 40}\right)^{1 / 2} \geq|\operatorname{ran}(z)|$.

Definição 4.4 Seja $X=\left(c_{00},\|\|.\right)$ um espaço normado sobre as sequências de suporte finito. Para todo $m \in \mathbb{N}$, definimos $A_{m}^{*}(X)$ como sendo o conjunto dos funcionais lineares da forma $f(m)^{-1} \sum_{i=1}^{m} f_{i}$ tais que,

1) $f_{1}<f_{2}<\ldots<f_{m}$.

2) $\left\|f_{i}\right\| \leq 1$ para todo $i$. 
Observação 4.5 Seja $X=\left(c_{00},\|\cdot\|\right)$ satisfazendo uma $f$-estimativa inferior então os funcionais pertencentes a $A_{m}^{*}(X)$ tem norma menor ou igual a um.

De fato, consideremos $\theta \in A_{m}^{*}(X) \operatorname{logo} \theta=f(m)^{-1} \sum_{i=1}^{m} f_{i}$, onde $f_{1}<\ldots<f_{m}$ e $\left\|f_{i}\right\| \leq 1$ para todo $i$. Tomemos $x \in X$ com $\|x\| \leq 1$ e calculemos $|\theta(x)|$. $|\theta(x)|=\left|f(m)^{-1} \sum_{i=1}^{m} f_{i}(x)\right| \leq f(m)^{-1} \sum_{i=1}^{m}\left|f_{i}(x)\right|$.

Seja $E_{i}=\operatorname{ran}\left(f_{i}\right)$ então $f(m)^{-1} \sum_{i=1}^{m}\left|f_{i}(x)\right|=f(m)^{-1} \sum_{i=1}^{m}\left|E_{i} f_{i}(x)\right|=$ $f(m)^{-1} \sum_{i=1}^{m}\left|f_{i}\left(E_{i} x\right)\right| \leq f(m)^{-1} \sum_{i=1}^{m}\left\|f_{i}\right\|\left\|E_{i} x\right\| \leq f(m)^{-1} \sum_{i=1}^{m}\left\|E_{i} x\right\| \leq\|x\|$.

Portanto $\|\theta\| \leq 1, \forall \theta \in A_{m}^{*}\left(X^{*}\right)$.

Definição 4.6 Seja $X=\left(c_{00},\|\cdot\|\right)$. Se $k \in \mathbb{N}$, seja $\Gamma_{k}^{X}$ o conjunto das sequências $g_{1}<$ $g_{2}<\ldots<g_{k}$ tais que $g_{i} \in Q$ para cada $i, g_{1} \in A_{j_{2 k}}^{*}(X)$ e $g_{i+1} \in A_{\sigma\left(g_{1}, \ldots, g_{i}\right)}^{*}(X)$ para qualquer $i, 1 \leq i \leq k-1$. Estas sequências são chamadas de sequências especiais.

Definição 4.7 Seja $X^{\prime}=\left(c_{00},\|\cdot\|\right)$, para todo $k \in \mathbb{N}$ definimos $B_{k}^{*}(X)$ como sendo o conjunto dos funcionais lineares da forma $f(k)^{-1 / 2} \sum_{j=1}^{k} g_{j}$ tal que $\left(g_{1}, \ldots, g_{k}\right) \in \Gamma_{k}^{X}$. Estes funcionais são chamados de funcionais especiais.

Observação 4.8 Se $\theta \in\left\{g_{i}\right\}_{i=1}^{k}$ onde $\left(g_{1}, \ldots, g_{k}\right) \in \Gamma_{k}^{X}$ então podemos escrever $\theta=\left(a_{i}\right)_{i}$ onde $\left(a_{i}\right)_{i} \in Q$ e portanto se $x=\left(b_{i}\right)_{i} \in c_{00}$ também podemos escrever $\theta(x)=\sum_{i=1}^{\infty} a_{i} b_{i}$.

Definiremos agora uma norma em $c_{00}$ como sendo o limite de uma sequência de normas .

Seja $X_{0}=\left(c_{00},\|.\|_{0}\right)$ onde $\|x\|_{0}=\|x\|_{\infty}$ e para $N \geq 0$ definimos $\|x\|_{X_{N+1}}=\|x\|_{X_{N}} \vee \sup \left\{f(n)^{-1} \sum_{i=1}^{n}\left\|E_{i} x\right\|_{X_{N}}: n \geq 2, E_{1}<\ldots<E_{n}\right\} \vee \sup \{|\bar{g}(E x)|:$ $\left.k \in K, \bar{g} \in B_{k}^{*}\left(X_{N^{\prime}}\right), E \subset \mathbb{N}\right\}$.

Observação Se $\|x\|_{X_{N}} \leq\|x\|_{X_{N+1}}$, para todo $x \in c_{00}$, então $B_{k}^{*}\left(X_{N}\right) \subset B_{k}^{*}\left(X_{N+1}\right)$. 
A demonstraçào de que $\|x\|_{X_{N}}$ é norma em $c_{00}$ é análoga a demonstração feita para o espaço de Tsirelson.

Vamos estudar agora algumas propriedades de $\left(\|x\|_{X_{N}}\right)_{N}$.

1) $\left(\|x\|_{X_{N}}\right)_{N}$ é uma sequência crescente de normas.

De fato, pela definição de $\|x\|_{X_{N+1}}$ temos $\|x\|_{X_{N+1}} \geq\|x\|_{X_{N}}$ para qualquer $N \geq 0$.

2) $\left(\|x\|_{X_{N}}\right)_{N}$ é limitada pela norma $l_{1}$.

De fato, seja $x=\left(x_{n}\right)_{n} \in c_{00}$ temos que $\|x\|_{0}=\sup _{n \in \mathbb{N}}\left|x_{n}\right| \leq \sum_{n=1}^{\infty}\left|x_{n}\right|=\|x\|_{l_{1}}$.

Suponhamos que $\|x\|_{X_{:}} \leq\|x\|_{l_{1}}$. Vamos provar que $\|x\|_{X_{N+1}} \leq\|x\|_{l_{1}}$. caso 1:

$\|x\|_{X_{N+1}}=\|x\|_{X_{N}} \leq\|x\|_{l_{1}}$.

caso 2:

$\|x\|_{X_{N+1}}=\sup \left\{f(n)^{-1} \sum_{i=1}^{n}\left\|E_{1} x\right\|_{X_{N}}: n \geq 2, E_{1}<\ldots<E_{n}\right\} \leq \sup \left\{\sum_{i=1}^{n}\left\|E_{i} x\right\|_{l_{1}}: n \geq\right.$ $\left.2, E_{1}<\ldots<E_{n}\right\} \leq\|x\|_{l_{1}}$

caso 3 :

$\|x\|_{X_{N+1}}=\sup \left\{|\bar{g}(E x)|: k \subseteq K, \bar{g} \in B_{k}^{*}\left(X_{N}\right), E \subset \mathbb{N}\right\}=\sup \left\{\left|f(k)^{-1 / 2} \sum_{j=1}^{k} g_{j}(E x)\right|:\right.$ $\left.k \in K, \bar{g} \in B_{k}^{*}\left(X_{N}\right), E \subset \mathbb{N}\right\} \leq \sup \left\{\left|\sum_{j=1}^{k} g_{j}(E x)\right|: k \in K, \bar{g} \in B_{k}^{*}\left(X_{N}\right), E \subset \mathbb{N}\right\} \leq$ $\sup \left\{\left|g_{1}(E x)\right|+\left|g_{2}(E x)\right|+\ldots+\left|g_{k}(E x)\right|: k \in K, \bar{g} \in B_{k}^{*}\left(X_{N}\right), E \subset \mathbb{N}\right\} \leq \sup \left\{\sum_{i \in E_{1} \cap E}\left|x_{i}\right|+\right.$ $\left.\sum_{i \in E_{2} \cap E}\left|x_{i}\right|+\ldots+\sum_{i \in E_{k} \cap E}\left|x_{i}\right|: k \in K, \bar{g} \in B_{k}^{*}\left(X_{N}\right), E \subset \mathbb{N}\right\} \leq\|x\|_{l_{1}}$ onde $E_{i}=\operatorname{ran}\left(g_{i}\right) \mathrm{e}$ como $g_{1}<\ldots<g_{k}$ temos $\operatorname{ran}\left(g_{1}\right)<\ldots<\operatorname{ran}\left(g_{k}\right)$.

Logo $\|x\|_{X_{N+1}} \leq\|x\|_{l_{1}}$. Assim temos que $\|x\|_{X_{N}} \leq\|x\|_{l_{1}}, \forall N \geq 0$.

Por 1) e 2) temos que existe $\lim _{N \rightarrow+\infty}\|x\|_{X_{N}}$. Definamos então $\|x\|=\lim _{N \rightarrow+\infty}\|x\|_{X_{N}} \mathrm{e}$ $X=\left(c_{00},\|\cdot\|\right)$.

Definição 4.9 O completado de $X=\left(c_{00},\|\|.\right)$ é o espaço de Gowers e Maurey, denotado por $X_{G M}$. 
A seguir faremos uma Proposição que nos dará uma forma implícita para a norma do espaço $X$.

Proposição 4.10 Para $x \in c_{00}$ e qualquer $N \geq 0$ temos,

a) $\|x\|_{X_{N+1}}=\sup \left\{f(n)^{-1} \sum_{i=1}^{n}\left\|E_{i} x\right\|_{X_{N}}: n \geq 2, E_{1}<\ldots<E_{n}\right\} \vee \sup \{|\bar{g}(E x)|: k \in K, \bar{g} \in$ $\left.B_{k}^{*}\left(X_{N}\right), E \subset \mathbb{N}\right\}$ ou $\|x\|_{X_{N+1}}=\|x\|_{\infty}$.

b) $\|x\|=\|x\|_{\infty} \vee \sup \left\{f(n)^{-1} \sum_{i=1}^{n}\left\|E_{i} x\right\|: n \geq 2, E_{1}<\ldots<E_{n}\right\} \vee \sup \{|\bar{g}(E x)|: k \in K, \bar{g} \in$ $\left.B_{k}^{*}(X), E \subset \mathbb{N}\right\}$.

\section{Demonstração:}

a) Sejam $x \in c_{00}$ e $N \geq 0$. Suponhamos que $\|x\|_{X_{N+1}}>\sup \left\{f(n)^{-1} \sum_{i=1}^{n}\left\|E_{i} x\right\|_{X_{N}}\right.$ : $\left.n \geq 2, E_{1}<\ldots<E_{n}\right\} \vee \sup \left\{|\bar{g}(E x)|: k \in K, \bar{g} \in B_{k}^{*}\left(X_{N}\right), E \subset \mathbb{N}\right\}$.

Então pela definição de $\|x\|_{X_{N+1}}$ temos que $\|x\|_{X_{N+1}}=\|x\|_{X_{N}}$. Segue assim que $\|x\|_{X_{N}}>\sup \left\{f(n)^{-1} \sum_{i=1}^{n}\left\|E_{i} x\right\|_{X_{N}}: n \geq 2, E_{1}<\ldots<E_{n}\right\} \vee \sup \{|\bar{g}(E x)|: k \in K, \bar{g} \in$ $\left.B_{k}^{*}\left(X_{N}\right), E \subset \mathbb{N}\right\} \geq \sup \left\{f(n)^{-1} \sum_{i=1}^{n}\left\|E_{i} x\right\|_{X_{N-1}}: n \geq 2, E_{1}<\ldots<E_{n}\right\} \vee \sup \{|\bar{g}(E x)|:$

$\left.k \in K, \bar{g} \in B_{k}^{*}\left(X_{N-1}\right), E \subset \mathbb{N}\right\}$.

E assim pela definição de $\|x\|_{X_{N}}$ temos que $\|x\|_{X_{N}}=\|x\|_{X_{N-1}}$.

Continuando o raciocínio teremos que $\|x\|_{X_{N+1}}=\|x\|_{X_{0}}=\|x\|_{\infty}$.

b) Análogo ao que fizemos para o espaço de Tsirelson temos $\|x\|_{X_{N+1}}=\|x\|_{\infty} \mathrm{V}$ $\sup \left\{f(n)^{-1} \sum_{i=1}^{n}\left\|E_{i} x\right\|_{X_{N}}: n \geq 2, E_{1}<\ldots<E_{n}\right\} \vee \sup \left\{|\bar{g}(E x)|: k \in K, \bar{g} \in B_{k}^{*}\left(X_{N}\right), E \subset\right.$ $\mathbb{N}$ \}. Como a função máximo é contínua passando o limite temos

$\lim _{N \rightarrow+\infty}\|x\|_{X_{N+1}}=\lim _{N \rightarrow+\infty}\|x\|_{\infty} \vee \lim _{N \rightarrow+\infty} \sup \left\{f(n)^{-1} \sum_{i=1}^{n}\left\|E_{i} x\right\|_{X_{N}}: n \geq 2, E_{1}<\ldots<E_{n}\right\} \vee$ $\lim _{N \rightarrow+\infty} \sup \left\{|\bar{g}(E x)|: k \in K, \bar{g} \in B_{k}^{*}\left(X_{N}\right), E \subset \mathbb{N}\right\}$.

Logo pela definição de $\|$.$\| temos$ $\|x\|=\|x\|_{\infty} \vee \sup \left\{f(n)^{-1} \sum_{i=1}^{n}\left\|E_{i} x\right\|: n \geq 2, E_{1}<\ldots<E_{n}\right\} \vee \sup \{|\bar{g}(E x)|: k \in K, \bar{g} \in$ $\left.B_{k}^{*}\left(X^{\prime}\right), E \subset \mathbb{N}\right\}, \forall x \in c_{00}$. 
Observação 4.11 Note que se permitíssimos que os $E_{i^{\prime} s}$ fossem subconjuntos finitos quaisquer de $\mathbb{N}$ e não necessariamente intervalos, então a base vetorial $\left(e_{n}\right)_{n}$ do espaço seria incondicional.

De fato, vamos assumir que os $E_{i^{\prime} s}$ possam ser subconjuntos e consideremos $A$ e $B$ subconjuntos finitos dos naturais com $A \subset B$.

Usando a definição implicita do espaço normado $X$ temos

1) $\left\|\sum_{n \in B} a_{n} e_{n}\right\|=\left\|\sum_{n \in B} a_{n} e_{n}\right\|_{\infty} \vee \sup \left\{f(N)^{-1} \sum_{i=1}^{N}\left\|E_{i} \sum_{n \in B} a_{n} e_{n}\right\|: N \geq 2, E_{1}<\ldots<E_{N}\right\} \vee$ $\sup \left\{\left|\bar{g}\left(E \sum_{n \in B} a_{n} e_{n}\right)\right|: k \in K, \bar{g} \in B_{k}^{*}(X), E \subset \mathbb{N}\right\}$.

2) $\left\|\sum_{n \in A} a_{n} e_{n}\right\|=\left\|\sum_{n \in A} a_{n} e_{n}\right\|_{\infty} \vee \sup \left\{f(N)^{-1} \sum_{i=1}^{N}\left\|E_{i} \sum_{n \in A} a_{n} e_{n}\right\|: N \geq 2, E_{1}<\ldots<E_{N}\right\} \vee$ $\sup \left\{\left|\bar{g}\left(E \sum_{n \in A} a_{n} e_{n}\right)\right|: k \in K, \bar{g} \in B_{k}^{*}(X), E \subset \mathbb{N}\right\}$.

Se $\left\|\sum_{n \in A} a_{n} e_{n}\right\|=\left\|\sum_{n \in A} a_{n} e_{n}\right\|_{\infty}$ temos que $\left\|\sum_{n \in A} a_{n} e_{n}\right\|_{\infty} \leq\left\|\sum_{n \in B} a_{n} e_{n}\right\|_{\infty} \leq\left\|\sum_{n \in B} a_{n} e_{n}\right\|$.

Se $\left\|\sum_{n \in A} a_{n} e_{n}\right\|=\sup \left\{f(N)^{-1} \sum_{i=1}^{N}\left\|E_{i} \sum_{n \in A} a_{n} e_{n}\right\|: N \geq 2, E_{1}<\ldots<E_{N}\right\}$ temos que $\sup \left\{f(N)^{-1} \sum_{i=1}^{N}\left\|E_{i} \sum_{n \in A} a_{n} e_{n}\right\|: N \geq 2, E_{1}<\ldots<E_{N}\right\} \leq \sup \left\{f(N)^{-1} \sum_{i=1}^{N}\left\|E_{i} \sum_{n \in B} a_{n} e_{n}\right\|:\right.$ $\left.N \geq 2, E_{1}<\ldots<E_{N}\right\} \leq\left\|\sum_{n \in B} a_{n} e_{n}\right\|$.

Se $\left\|\sum_{n \in A} a_{n} e_{n}\right\|=\sup \left\{\left|\bar{g}\left(E \sum_{n \in A} a_{n} e_{n}\right)\right|: k \in K, \bar{g} \in B_{k}^{*}(X), E \subset \mathbb{N}\right\}$ temos que $\sup \left\{\left|\bar{g}\left(E \sum_{n \in A} a_{n} e_{n}\right)\right|: k \in K, \bar{g} \in B_{k}^{*}(X), E \subset \mathbb{N}\right\} \leq \sup \left\{\left|\bar{g}\left(E \sum_{n \in B} a_{n} e_{n}\right)\right|: k \in K, \bar{g} \in\right.$ $\left.B_{k}^{*}(X), E \subset \mathbb{N}\right\} \leq\left\|\sum_{n \in B} a_{n} e_{n}\right\|$

Portanto $\left\|\sum_{n \in A} a_{n} e_{n}\right\| \leq\left\|\sum_{n \in B} a_{n} e_{n}\right\|$ para subconjuntos finitos dos naturais com $A \subset B$.

Logo pelo Teorema 0.5 temos que a inequação acima implica que $\left(e_{n}\right)_{n}$ é base incondicional para o espaço. Por isso só consideraremos intervalos para formar a norma do espaço de Gowers e Maurey. 
Passaremos a enunciar os Lemas ( bastante técnicos ) necessários para demonstrarmos que o espaço é hereditariamente indecomponível. O leitor perceberá que a demonstração de cada Lema dependerá do Lema anterior.

As técnicas utilizadas no último Lema serão de grande importância para a demonstração do principal resultado desta dissertação, isto é, Teorema 4.17.

Lema 4.12 Se $N \in J \backslash K_{0}^{\prime}$ então $G(x)=x f^{-1}(x)$ para todo $x$ no intervalo $[\ln N$, exp $N]$, onde $K_{0} \subset K$ é um subconjunto dos naturais e $G$ é a função construída na Observação 2.10.

\section{Demonstração:}

Sejam $k, l \in K_{0} \cup\{1\}$ o maior e o menor número respectivamente tal que $k<N$ e $l>N$.

Temos que $(k !)^{4}<f^{-1}\left(f(l)^{1 / 2}\right)$. De fato, sabemos que $\ln \ln N<\exp \exp N$ e vamos verificar que 1) $(k !)^{4}<\ln \ln N$ e 2) $f^{-1}\left(f(l)^{1 / 2}\right)>\operatorname{expexp} N$.

1) $(k !)^{4}<\ln \ln N \Longleftrightarrow 4 \ln k !<\ln \ln \ln N$. Mas $4 k^{2} \leq \ln \ln \ln N$. logo basta demonstrarmos que $4 \ln k !<4 k^{2}$, isto é, $k !<e^{k^{2}}$. Por indução temos, $k=1,1<e, k=2.2<e^{4}$.

Suponha que é verdadeiro para $k \leq n$. Vamos provar que é verdadeiro para $n+1$. $(n+1) !=(n+1) n !<(n+1) e^{n^{2}}<e^{(n+1)^{2}}$, pois $e^{(n+1)^{2}}=e^{n^{2}+2 n+1}=e^{n^{2}} \cdot e^{2 n+1}$, e como vamos verificar $(n+1)<e^{2 n+1}$.

Temos que $n+1 \leq 2 n+1$ e $e>2$ portanto $n+1<e^{2 n+1}$. Portanto $k !<e^{k^{2}}$.

2) $e^{e^{N}}<f^{-1}\left(f(l)^{1 / 2}\right) \Longleftrightarrow f\left(e^{e^{N}}\right)<f(l)^{1 / 2}$, isto é, $\log _{2}\left(1+e^{e^{N}}\right)<\left(\log _{2}(1+l)\right)^{1 / 2}$. Como $e^{e^{e^{4 N^{2}}}} \leq l \leq l+1$ segue que $\log _{2}(e)^{e^{e^{4 N^{2}}}}<\log _{2}(l+1)$, ou seja, $e^{e^{4 N^{2}}} \leq$ $e^{e^{4 N^{2}}} \log _{2} e<\log _{2}(l+1)$ e portanto $f\left(e^{e^{N}}\right)<e^{e^{N}} \leq e^{e^{2 N^{2}}}<\left(\log _{2}(l+1)\right)^{1 / 2}$.

Seja $x$ com $(k !)^{4}<x<f^{-1}\left(f(l)^{1 / 2}\right)$, logo $f(x)<f(l)^{1 / 2}$. Como $(k !)^{4}<x<$ $f^{-1}\left(f(l)^{1 / 2}\right)$ temos que $x \notin K_{0}$, pois se $x \in K_{0}$ então $x \leq k$ ou $l \leq x$.

No primeiro caso teríamos $x \leq k \leq(k !)^{4}$, absurdo.

No segundo caso teríamos $f(l)^{1 / 2} \leq f(l) \leq f(x)$, absurdo. 
Sabemos pela Observação 2.10 que $h(x)=\inf A$, onde $A=\left\{\phi\left(x_{1}\right) \phi\left(x_{2}\right) \ldots \phi\left(x_{m}\right)\right.$ : $\left.x_{1} x_{2} \ldots x_{m} \geq x\right\}$. Como $\phi(x) \in A$, segue que $h(x) \leq \phi(x)=f(x)$ pela definição de $\phi$ dada na Observação 2.10. Como $\phi(1)=1$, podemos assumir que todos os $x_{i^{\prime} s}$, com $x_{1} x_{2} \ldots x_{m} \geq x$ são diferentes de 1 .

Observação 1: $h(x)=\inf B$, onde $B=\left\{\phi\left(x_{1}\right) \phi\left(x_{2}\right) \ldots \phi\left(x_{m}\right): x_{1} x_{2} \ldots x_{m} \geq x\right.$, e tal que existe no máximo um $\left.j \operatorname{com} x_{j} \notin K_{0}\right\}$. Pois mostraremos que dado $a \in A, \exists b \in B$, com $b \leq a$.

De fato, se $x_{1}$ e $x_{2}$. por exemplo. não pertencem a $K_{0}$, então $\phi\left(x_{1}\right) \phi\left(x_{2}\right) \phi\left(x_{3}\right) \ldots \phi\left(x_{m}\right)=$ $f\left(x_{1}\right) f\left(x_{2}\right) \phi\left(x_{3}\right) \ldots \phi\left(x_{m}\right) \geq f\left(x_{1} x_{2}\right) \phi\left(x_{3}\right) \ldots \phi\left(x_{m}\right)$.

caso 1: $x_{1} x_{2} \in K_{0}$.

Logo $\phi\left(x_{1} x_{2}\right)=f\left(x_{1} x_{2}\right)^{1 / 2}$ e portanto $f\left(x_{1} x_{2}\right) \phi\left(x_{3}\right) \ldots \phi\left(x_{m}\right) \geq f\left(x_{1} x_{2}\right)^{1 / 2} \phi\left(x_{3}\right) \ldots \phi\left(x_{m}\right)=$ $\phi\left(x_{1} x_{2}\right) \phi\left(x_{3}\right) \ldots \phi\left(x_{m}\right)$. isto é. $\phi\left(x_{1}\right) \phi\left(x_{2}\right) \phi\left(x_{3}\right) \ldots \phi\left(x_{m}\right) \geq \phi\left(x_{1} x_{2}\right) \phi\left(x_{3}\right) \ldots \phi\left(x_{m}\right)$.

caso 2: $x_{1} x_{2} \notin K_{0}$.

Logo $\phi\left(x_{1} x_{2}\right)=f\left(x_{1} x_{2}\right)$ e portanto $f\left(x_{1} x_{2}\right) \phi\left(x_{3}\right) \ldots \phi\left(x_{m}\right)=\phi\left(x_{1} x_{2}\right) \phi\left(x_{3}\right) \ldots \phi\left(x_{m}\right)$, isto é, $\phi\left(x_{1}\right) \phi\left(x_{2}\right) \phi\left(x_{3}\right) \ldots \phi\left(x_{m}\right) \geq \phi\left(x_{1} x_{2}\right) \phi\left(x_{3}\right) \ldots \phi\left(x_{m}\right)$.

Procedendo analogamente com outros pares de pontos que eventualmente não pertençam a $K_{0}^{\prime}$, pode se supor que $\phi\left(x_{1}\right) \phi\left(x_{2}\right) \phi\left(x_{3}\right) \ldots \phi\left(x_{m}\right) \geq \phi\left(x_{1}^{\prime}\right) \phi\left(x_{2}^{\prime}\right) \phi\left(x_{3}^{\prime}\right) \ldots \phi\left(x_{m}^{\prime}\right)$ com $x_{1}^{\prime} x_{2}^{\prime} x_{3}^{\prime} \ldots x_{m}^{\prime} \geq x$ e com no máximo um $\operatorname{dos} x_{i}^{\prime} \notin \Lambda_{0}^{\prime}$.

Observação 2: $h(x)=\inf C$, onde $C=\left\{\phi\left(x_{1}\right) \phi\left(x_{2}\right) \ldots \phi\left(x_{m}\right): x_{1} x_{2} \ldots x_{m} \geq x\right.$, e tal que existe no máximo um $j \operatorname{com} x_{j} \notin K_{0}$ e não existe $r, s, t$ tal que $\left.x_{r}=x_{s}=x_{t} \in K_{0}\right\}$. Pois mostraremos que dado $b \in B$, existe $c \in C$, com $c \leq b$.

Primeiro vamos verificar que $\forall p \in K, f(p)^{3 / 2}>f\left(p^{3}\right)$.

Consideremos $D(x)=\left(\log _{2}(x+1)\right)^{3 / 2}-\log _{2}\left(x^{3}+1\right)$. Vamos verificar que $D(x)>0$. $D(1048576)=\left(\log _{2}(1048576+1)\right)^{3 / 2}-\log _{2}\left((1048576)^{3}+1\right)>0$, pois $\left(\log _{2}(1048576+\right.$ $1))^{3 / 2}-\log _{2}\left((1048576)^{3}+1\right)>\left(\log _{2} 1048576\right)^{3 / 2}-\log _{2}\left((1048576)^{3}\right),\left(\log _{2} 1048576\right)^{3 / 2}>$ 89 e $\log _{2}\left((1048576)^{3}\right)=60$.

$D^{\prime}(x)=\frac{3 \log _{2} e}{x+1}\left(\frac{\left(\log _{2}(x+1)\right)^{1 / 2}}{2}-\frac{x^{2}}{x^{2}-x+1}\right)$. Seja $H(x)=\frac{\left(\log _{2}(x+1)\right)^{1 / 2}}{2}-\frac{x^{2}}{x^{2}-x+1}$.

$H(65535)>0$, pois $\frac{\left(\log _{2}(65535+1)\right)^{1 / 2}}{2}=2$ e $\frac{(65535)^{2}}{(65535)^{2}-65535+1}<1,1$.

$H^{\prime}(x)=\frac{\log _{2} e}{4(x+1)\left(\log _{2}(x+1)\right)^{i / 2}}+\frac{x^{2}}{\left(x^{2}-x+1\right)^{2}}-\frac{2 x}{\left(x^{2}-x+1\right)^{2}}$.

$H^{\prime}(x)>0 \Longleftrightarrow \frac{x^{2}-2=}{\left(x^{2}-x+1\right)^{2}}>0 \Longleftrightarrow x^{2}-2 x>0 \Longleftrightarrow x<0$ ou $x>2$. 
Como estamos trabalhando com valores maiores do que dois temos o resultado, ou seja, $H$ é uma função positiva logo $D$ é uma função positiva.

Então, se por exemplo, $x_{1}=x_{2}=x_{3} \in K_{0}$ temos $\phi\left(x_{1}\right) \phi\left(x_{2}\right) \phi\left(x_{3}\right) \phi\left(x_{4}\right) \ldots \phi\left(x_{m}\right)=$ $f\left(x_{1}\right)^{1 / 2} f\left(x_{1}\right)^{1 / 2} f\left(x_{1}\right)^{1 / 2} \phi\left(x_{4}\right) \ldots \phi\left(x_{m}\right)=f\left(x_{1}\right)^{3 / 2} \phi\left(x_{4}\right) \ldots \phi\left(x_{m}\right)>f\left(x_{1}^{3}\right) \phi\left(x_{4}\right) \ldots \phi\left(x_{m}\right)$.

Verificaremos agora que se $p \in J$ então $p^{3} \notin J$.

De fato, se $p$ e $p^{3}$ estão em $J$, então como $p<p^{3}$ temos que ter $\ln \ln \ln p^{3} \geq 4 p^{2}$. Ora $\ln \ln \ln p^{3} \leq \ln \ln p^{3} \leq \ln p^{3}$ e $\ln p^{3} \leq 4 p^{2}$ como veremos.

De fato, $\ln p^{3} \leq 4 p^{2} \Longleftrightarrow 3 \ln p \leq 4 p^{2}$, o que é verdade para $p \geq 1$.

Portanto não temos $\ln \ln \ln p^{3} \geq 4 p^{2}, \operatorname{logo} p^{3} \notin J$.

Em particular, como $x_{1} \in K_{0}$ temos que $x_{1}^{3} \notin K_{0}$. logo $\phi\left(x_{1}^{3}\right)=f\left(x_{1}^{3}\right)$. Consequentemente $\phi\left(x_{1}\right) \phi\left(x_{2}\right) \phi\left(x_{3}\right) \phi\left(x_{4}\right) \ldots \phi\left(x_{m}\right)>\phi\left(x_{1}^{3}\right) \phi\left(x_{4}\right) \ldots o\left(x_{m}\right)$.

Procedendo analogamente com outras eventuais triplas e também usando o que foi feito na Observação 1 teremos provado o desejado.

Observação $3: h(x)=\inf D$, onde $D=\left\{\phi\left(x_{1}\right) \dot{o}\left(x_{2}\right) \ldots \phi\left(x_{m}\right): x_{1} x_{2} \ldots x_{m} \geq x\right.$, e tal que existe no máximo um $j \operatorname{com} x_{j} \notin K_{0}$ e não existe $r, s, t$ tal que $x_{r}=x_{s}=x_{t} \in K_{0}$ e se $x_{j} \in K_{0}$ então $\left.x_{j} \leq k\right\}$.

Basta demonstrarmos que se $x_{1} x_{2} \ldots x_{m} \geq x$, com algum $x_{j} \in K_{0}^{\prime}$ e $k<x_{j}$ então $\phi(x)<\phi\left(x_{1}\right) \phi\left(x_{2}\right) \ldots \phi\left(x_{m}\right)$ e $\phi(x) \in D$.

De fato, como $x<f^{-1}\left(f(l)^{1 / 2}\right)$ segue que $f(x)<f(l)^{1 / 2}$ e como $k<x_{j}$, então pela escolha de $l$ concluímos que $l \leq x_{j}$, portanto $o(x)=f(x)<f(l)^{1 / 2} \leq f\left(x_{j}\right)^{1 / 2} \leq$ $\phi\left(x_{1}\right) \ldots \phi\left(x_{j}\right) \ldots \phi\left(x_{m}\right)$ e por outro lado como $x \notin K_{0}$ então $\phi(x) \in D$.

Sejam $x_{1}, x_{2}, \ldots, x_{m}$ elementos de $K_{0}, \operatorname{com} x_{j} \leq k, \forall j, 1 \leq j \leq m$ e tal que não existe $r, s, t \operatorname{com} x_{r}=x_{s}=x_{t} \in K_{0}$ então $x>x_{1} x_{2} \ldots x_{m}$.

De fato, temos $x_{1} x_{2} \ldots x_{m} \leq 2^{2} 3^{2} \ldots k^{2}=(k !)^{2}<(k !)^{4}<x$.

Da observação acima e da Observação 3 concluímos que $h(x)=\inf E$, onde $E=\left\{\phi\left(x_{1}\right) \phi\left(x_{2}\right) \ldots \phi\left(x_{m}\right): x_{1} x_{2} \ldots x_{m} \geq x\right.$, e tal que existe exatamente um $x_{j} \notin K_{0}$ e não existe $r, s, t$ tal que $x_{r}=x_{s}=x_{t} \in K_{0}$ e se $x_{j} \in K_{0}$. então $\left.x_{j} \leq k\right\}$.

Seja $\phi\left(x_{1}\right) \phi\left(x_{2}\right) \ldots \phi\left(x_{m}\right)$ um elemento de $E$ e, por exemplo, $x_{1}$ o único elemento que 
não pertence a $K_{0}$. Suponhamos que $m>1$.

Como $x_{2} \ldots x_{m} \leq(k !)^{2} \leq x^{1 / 2}$, temos que $x_{1} \geq x^{1 / 2}$ e portanto $\phi\left(x_{1}\right) \phi\left(x_{2}\right) \ldots \phi\left(x_{m}\right)=$ $f\left(x_{1}\right) \phi\left(x_{2}\right) \ldots \dot{\phi}\left(x_{m}\right) \geq f\left(x^{1 / 2}\right) f(\min K)^{1 / 2}$.

Como $f\left(j_{1}\right) \geq 256$, segue que $f(\min K)^{1 / 2} \geq 16$ e portanto $\phi\left(x_{1}\right) \phi\left(x_{2}\right) \ldots \phi\left(x_{m}\right) \geq$ $6 f\left(x^{1 / 2}\right)>f(x)=\phi(x)$.

De fato $6 f\left(x^{1 / 2}\right)>f(x)$, pois considere $D(x)=6 f\left(x^{1 / 2}\right)-f(x)$.

$D(1)=5>0$ e $D^{\prime}(x)=\log _{2} e\left(\frac{3}{x^{1 / 2}+x}-\frac{1}{x+1}\right)$. Vamos verificar que $D^{\prime}(x)>0$.

$\frac{3}{x^{1 / 2}+x}-\frac{1}{x+1}>0 \Longleftrightarrow 3 x+3>x^{1 / 2}+x \Longleftrightarrow 2 x>x^{1 / 2}-3$ o que é verdade pois $\sqrt{x}<x<2 x$ e como $\sqrt{x}-3<\sqrt{x}<2 x$ temos o que queríamos.

Portanto $D$ é uma função positiva, $\log$ o $6 f\left(x^{1 / 2}\right)>f(x)$.

Portanto qualquer elemento de $E$ é maior que $\phi(x)$ e como $x \notin K_{0}$ temos que $\phi(x) \in$ $E, \operatorname{logo} \inf E=\phi(x)=f(x)$.

Sabemos agora que $H(x)=\frac{x}{f(x)}, \forall x \in\left((k !)^{4}, f^{-1}\left(f(l)^{1 / 2}\right)\right)$ e, em particular, para todo $x$ no intervalo $[\ln \ln N, \exp \exp N]$. Temos que $[\ln N, \exp N] \subset[\ln \ln N, \exp \exp N]$. Consideremos $x_{0} \in[\ln N, \exp N]$.

Teremos $G\left(x_{0}\right)=\frac{x_{0}}{f\left(x_{0}\right)}$ se a reta tangente $r(x)$ ao gráfico de $\frac{x}{f(x)}$ no ponto $\left(x_{0}, \frac{x_{0}}{f\left(x_{0}\right)}\right)$ for pelo menos maior que $\frac{x}{\sqrt{f(x)}}$ para todo $x$ positivo fora do intervalo $[\ln \ln N, \exp \exp N]$.

De fato, como $H(x)=\frac{x}{f(x)}$ é concava no intervalo $[\ln \ln N, \operatorname{expexp} N]$ temos que $\sup \left\{\lambda_{1} H\left(x_{1}\right)+\lambda_{2} H\left(x_{2}\right)+\ldots+\lambda_{n} H\left(x_{n}\right): \lambda_{1} x_{1}+\ldots+\lambda_{n} x_{n}=x_{0}, \sum_{i=1}^{n} \lambda_{i}=1\right.$ e $x_{1}, x_{2}, \ldots, x_{m} \in$

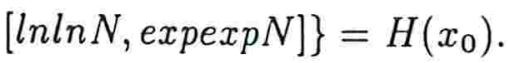

Por outro lado seja $x \in \mathbb{R}_{+}$, como $f(x)^{1 / 2}$ é submultiplicativa e $f(x)^{1 / 2} \leq \phi(x)$ segue pela definição de $h$ que $f(x)^{1 / 2} \leq h(x)$ e portanto $H(x)=\frac{x}{h(x)} \leq \frac{x}{f(x)^{1 / 2}}$.

Se $\frac{x}{f(x)^{1 / 2}} \leq r(x), \forall x$ fora do intervalo $[\ln \ln N, \exp \exp N]$, então $H(x) \leq r(x), \forall x$ fora do intervalo $[\ln \ln N, \exp \exp N]$ e portanto se $\lambda_{1} x_{1}+\ldots+\lambda_{n} x_{n}=x_{0}$ teremos $\lambda_{1} H\left(x_{1}\right)+$ $\lambda_{2} H\left(x_{2}\right)+\ldots+\lambda_{n} H\left(x_{n}\right) \leq \lambda_{1} r\left(x_{1}\right)+\lambda_{2} r\left(x_{2}\right)+\ldots+\lambda_{n} r\left(x_{n}\right)$.

$r$ é uma reta logo $r(x)=a x+b$. Temos que $\lambda_{1} x_{1}+\ldots+\lambda_{n} x_{n}=x_{0}$ e $\sum_{i=1}^{n} \lambda_{i}=1$. $\operatorname{logo}$ $\lambda_{1} r\left(x_{1}\right)+\lambda_{2} r\left(x_{2}\right)+\ldots+\lambda_{n} r\left(x_{n}\right)=\lambda_{1}\left(a x_{1}+b\right)+\lambda_{2}\left(a x_{2}+b\right)+\ldots+\lambda_{n}\left(a x_{n}+b\right)=$ $a\left(\lambda_{1} x_{1}+\lambda_{2} x_{2}+\ldots+\lambda_{n} x_{n}\right)+b\left(\lambda_{1}+\lambda_{2}+\ldots+\lambda_{n}\right)=a x_{0}+b=r\left(x_{0}\right)=H\left(x_{0}\right)$. 
Portanto $\sup \left\{\lambda_{1} H\left(x_{1}\right)+\lambda_{2} H\left(x_{2}\right)+\ldots+\lambda_{n} H\left(x_{n}\right): \lambda_{1} x_{1}+\ldots+\lambda_{n} x_{n}=x_{0}, \sum_{i=1}^{n} \lambda_{i}=\right.$
$1\}=H\left(x_{0}\right)=\frac{x_{0}}{f\left(x_{0}\right)}$, isto é, $G\left(x_{0}\right)=H\left(x_{0}\right)=\frac{x_{0}}{f\left(x_{0}\right)}$.

Cálculo de $r(x)$ :

$\left(\frac{x}{f(x)}\right)^{\prime}=\frac{f(x)-x f^{\prime}(x)}{f(x)^{2}}$, com $f^{\prime}(x)=\frac{\log _{2} e}{x+1}$. Logo, $r(x)=\frac{x_{0}}{f\left(x_{0}\right)}+\frac{f\left(x_{0}\right)-x_{0} f^{\prime}\left(x_{0}\right)}{f\left(x_{0}\right)^{2}}\left(x-x_{0}\right)$, isto é, $r(x)=\frac{x_{0}}{f\left(x_{0}\right)}+\frac{1}{f\left(x_{0}\right)}\left(1-\frac{x_{0} \log _{2} e}{\left(1+x_{0}\right) \log _{2}\left(x_{0}+1\right)}\right)\left(x-x_{0}\right)$.

Então $r(x)=\frac{x_{0}}{f\left(x_{0}\right)}+\frac{1}{f\left(x_{0}\right)}\left(1-\frac{x_{0}}{\left(1+x_{0}\right) \ln \left(x_{0}+1\right)}\right)\left(x-x_{0}\right)$.

Como o coeficiente angular é positivo a reta tangente é estritamente crescente.

Quando $x \geq 0$, tem-se $r(x) \geq r(0)$,

$r(0)=\frac{x_{0}}{f\left(x_{0}\right)}+\frac{1}{f\left(x_{0}\right)}\left(1-\frac{x_{0} \log _{2} e}{\left(1+x_{0}\right) \log _{2}\left(x_{0}+1\right)}\right)\left(-x_{0}\right)=\frac{x_{0}^{2}}{\log _{2}\left(x_{0}+1\right)\left(x_{0}+1\right) \ln \left(x_{0}+1\right)}=\frac{x_{0}^{2} \ln 2}{\left(x_{0}+1\right)\left(\ln \left(x_{0}+1\right)\right)^{2}}$.

$\operatorname{Mas} \frac{x_{0}^{2} \ln 2}{\left(x_{0}+1\right)\left(\ln \left(x_{0}+1\right)\right)^{2}} \geq \frac{x_{0}}{2\left(\log _{2}\left(x_{0}+1\right)\right)^{2}}$, pois $\frac{x_{0}^{2} \ln 2}{\left(x_{0}+1\right)\left(\ln \left(x_{0}+1\right)\right)^{2}} \geq \frac{x_{0}}{2\left(\log _{2}\left(x_{0}+1\right)\right)^{2}} \Longleftrightarrow$

$\frac{x_{0}^{2} \ln 2}{\left(x_{0}+1\right)\left(\ln \left(x_{0}+1\right)\right)^{2}} \geq \frac{x_{0}(\ln 2)^{2}}{2\left(\ln \left(x_{0}+1\right)\right)^{2}} \Longleftrightarrow \frac{x_{0}^{2}}{\left(x_{0}+1\right)} \geq \frac{x_{0} \ln 2}{2} \Longleftrightarrow \frac{2 x_{0}}{x_{0}+1} \geq \ln 2 \Longleftrightarrow e^{\frac{2 x_{0}}{x_{0}+1}} \geq 2$, o que é verdade pois $\frac{2 x_{0}}{x_{0}+1} \geq 1$.

Para $x_{0} \geq \ln N$ ( que é o caso) e $x \leq \ln \ln N \leq \ln N \leq x_{0}$ ( que é o caso), temos $\frac{x}{f(x)^{1 / 2}} \leq \frac{x_{0}}{2 f\left(x_{0}\right)^{2}}$.

De fato, como $x \leq \ln \ln N$ e $x_{0} \geq \ln N$ temos $x \leq \ln \ln N \leq \ln x_{0}$, isto é, $e^{x} \leq x_{0}$.

Afirmação: $f(x) \leq x^{1 / 4}, \forall x \geq 32^{4}$, em particular, $f(x) \leq\left(\frac{x}{2}\right)^{1 / 2}, \forall x \geq 32^{4}$, pois $x^{1 / 4} \leq\left(\frac{x}{2}\right)^{1 / 2} \Longleftrightarrow x^{1 / 2} \leq \frac{x}{2} \Longleftrightarrow 2 \leq x^{1 / 2}$, o que é verdade já que $x \geq 32^{4}$.

Seja $d(x)=x^{1 / 4}-f(x)$, vamos provar que $d(x)>0$. $d\left(32^{4}\right)=32-\log _{2}\left(32^{4}+1\right)>0 \Longleftrightarrow \log _{2}\left(32^{4}+1\right)<32 \Longleftrightarrow 32^{4}+1<2^{32}$, mas $1+32^{4}<32^{5}=\left(2^{5}\right)^{5}<2^{32}$. Portanto $d\left(32^{4}\right)>0$.

$d^{\prime}(x)=\frac{1}{4 x^{3 / 4}}-\frac{\log _{2} e}{x+1}>0 \Longleftrightarrow \frac{1}{4 x^{3 / 4}}>\frac{\log _{2} e}{x+1} \Longleftrightarrow \frac{1+x}{x^{3 / 4}}>4 \log _{2} e$. Como $4 \log _{2} e<8$, basta demonstrar que $\frac{1+x}{x^{3 / 4}}>8$, isto é, $1+x>8 x^{3 / 4}$. Seja $D(x)=1+x-8 x^{3 / 4}$.

$D\left(32^{4}\right)=1+32^{4}-8\left(32^{4}\right)^{3 / 4}=1+32^{4}-8\left(32^{3}\right)>0$.

$D^{\prime}(x)=1-\frac{6}{x^{1 / 4}}>0 \Longleftrightarrow 6<x^{1 / 4}$ o que é verdade porque $x \geq 32^{4} \operatorname{logo} x^{1 / 4}>32$.

Portanto $f(x) \leq x^{1 / 4}, \forall x \geq 32^{4}$.

Queremos provar que $\frac{x}{f(x)^{1 / 2}} \leq \frac{x_{0}}{2 f\left(x_{0}\right)^{2}}$. Vamos fazer $c=\frac{x_{0}}{2 f\left(x_{0}\right)^{2}}$. Quero provar que $\frac{x}{f(x)^{1 / 2}} \leq c$, isto é. $x \leq c f(x)^{1 / 2}$, isto é. $x^{2} \leq c^{2} f(x)$. 
Seja $D(x)=c^{2} f(x)-x^{2}$. Quero provar que $D(x) \geq 0$.

$D(1)=\left(\frac{x_{0}}{2 f\left(x_{0}\right)^{2}}\right)^{2}-1$.

$D(1) \geq 0 \Longleftrightarrow\left(\frac{x_{0}}{2 f\left(x_{0}\right)^{2}}\right)^{2} \geq 1 \Longleftrightarrow \frac{x_{0}}{2 f\left(x_{0}\right)^{2}} \geq 1$, isto é, $\frac{x_{0}}{2} \geq f\left(x_{0}\right)^{2} \Longleftrightarrow\left(\frac{x_{0}}{2}\right)^{1 / 2} \geq f\left(x_{0}\right)$, o que é verdade pela afirmação, já que $x_{0} \geq 32^{4}$.

$D^{\prime}(x)=c^{2} \frac{\log _{2} e}{x+1}-2 x$.

$D^{\prime}(x)>0 \Longleftrightarrow c^{2} \frac{\log _{2} e}{x+1}-2 x>0 \Longleftrightarrow c^{2} \frac{\log _{2} e}{x+1}>2 x \Longleftrightarrow c^{2} \log _{2} e>(x+1) 2 x$.

Como $\log _{2} e>1$, basta mostrar que $2 x(x+1)<c^{2}$, isto é. $2 x(x+1)<\left(\frac{x_{0}}{2 f\left(x_{0}\right)^{2}}\right)^{2}=$ $\left(\frac{x_{0}^{1 / 4}}{f\left(x_{0}\right)}\right)^{2}\left(\frac{x_{0}^{1 / 4}}{f\left(x_{0}\right)}\right)^{2} \frac{x_{0}}{4}$. Como $x_{0} \geq 32^{4}$, segue pela afirmação que $1 \leq \frac{x_{0}^{1 / 4}}{f\left(x_{0}\right)}$.

Logo basta provarmos que $2 x(x+1)<\frac{x_{0}}{4}$, isto é, $8 x(x+1)<x_{0}$.

Usando derivada é fácil verificar que $8 x<e^{\frac{x}{2}}, \forall x \geq 14$ e $x+1<e^{\frac{x}{2}}, \forall x \geq 6$.

Vamos separar a demonstração de que $8 x(x+1)<x_{0}$ em dois casos.

caso 1: $x<14$.

Então $8 x(x+1)<\sqrt{\ln N} \sqrt{\ln N}=\ln N \leq x_{0}$.

caso $2: x \geq 14$.

Então $8 x(x+1) \leq e^{\frac{x}{2}} e^{\frac{x}{2}}=e^{x} \leq x_{0}$. Portanto $8 x(x+1) \leq x_{0} . \forall x$.

Logo $2 x(x+1)<c^{2}$, portanto $\frac{x}{f(x)^{1 / 2}} \leq c$ como queríamos.

Quando $x \geq 2 x_{0}$, note que $2 x_{0} \leq 2 \exp N \leq \exp \exp N$, temos $r(x) \geq \frac{x}{4 f\left(x_{0}\right)}$.

De fato, considere $D(x)=r(x)-\frac{x}{4 f\left(x_{0}\right)}=\frac{x_{0}}{f\left(x_{0}\right)}+\frac{1}{f\left(x_{0}\right)}\left(1-\frac{x_{0}}{\left(1+x_{0}\right) \ln \left(x_{0}+1\right)}\right)\left(x-x_{0}\right)-\frac{x}{4 f\left(x_{0}\right)}$.

Quero provar que $D^{\prime}(x) \geq 0$.

$D^{\prime}(x)=\frac{1}{f\left(x_{0}\right)}-\frac{x_{0}}{f\left(x_{0}\right)\left(x_{0}+1\right) \ln \left(x_{0}+1\right)}-\frac{1}{4 f\left(x_{0}\right)}=\frac{3}{4 f\left(x_{0}\right)}-\frac{x_{0}}{f\left(x_{0}\right)\left(x_{0}+1\right) \ln \left(x_{0}+1\right)}>0 \Longleftrightarrow \frac{x_{0}}{\left(x_{0}+1\right) \ln \left(x_{0}+1\right)}<$ $\frac{3}{4}$. Temos que, $\frac{x_{0}}{\left(x_{0}+1\right)} \frac{1}{\ln \left(x_{0}+1\right)}<\frac{1}{\ln \left(x_{0}+1\right)}$ e $\frac{1}{\ln \left(x_{0}+1\right)}<\frac{3}{4} \Longleftrightarrow \frac{4}{3}<\ln \left(x_{0}+1\right)$ o que é verdade pois $\frac{4}{3}<\ln \ln N<\ln x_{0}<\ln \left(x_{0}+1\right)$.

$D\left(2 x_{0}\right)=\frac{x_{0}}{f\left(x_{0}\right)}+\frac{x_{0}}{f\left(x_{0}\right)}-\frac{x_{0}^{2}}{f\left(x_{0}\right)\left(x_{0}+1\right) \ln \left(x_{0}+1\right)}-\frac{2 x_{0}}{4 f\left(x_{0}\right)}=\frac{3 x_{0}}{2 f\left(x_{0}\right)}-\frac{x_{0}^{2}}{f\left(x_{0}\right)\left(x_{0}+1\right) \ln \left(x_{0}+1\right)}>0 \Longleftrightarrow$ $\frac{x_{0}}{\left(x_{0}+1\right) \ln \left(x_{0}+1\right)}<\frac{3}{2}$.

$\frac{x_{0}}{\left(x_{0}+1\right)} \frac{1}{\ln \left(x_{0}+1\right)}<\frac{1}{\ln \left(x_{0}+1\right)}<\frac{3}{2} \Longleftrightarrow \ln \left(x_{0}+1\right)>\frac{2}{3}$, mas $\frac{2}{3}<\ln \ln N<\ln x_{0}<\ln \left(x_{0}+1\right)$.

Para $x \geq \exp \operatorname{expN}$ ( que é o caso ) a condição $x_{0} \leq \operatorname{expN}$ ( que é o caso ) garante que $\frac{x}{f(x)^{1 / 2}} \leq \frac{x}{4 f\left(x_{0}\right)}$.

De fato, $\left(4 f\left(x_{0}\right)\right)^{2} \leq\left(f(x)^{1 / 2}\right)^{2}$, ou seja, $16\left(f\left(x_{0}\right)\right)^{2} \leq f(x)$ pois $16 f\left(x_{0}\right)^{2}=16\left(\log _{2}\left(x_{0}+\right.\right.$ 
$+1))^{2} \leq 16\left(\log _{2}\left(\epsilon^{N}+1\right)\right)^{2} \log$ basta mostrar que $16\left(\log _{2}\left(e^{N}+1\right)\right)^{2} \leq \log _{2}\left(e^{e^{N}}+1\right)$, pois $\log _{2}\left(e^{e^{N}}+1\right) \leq \log _{2}(x+1)=f(x)$.

Isto é, basta mostrar que $16\left(\log _{2}\left(e^{N}+1\right)\right)^{2} \leq \log _{2} 2^{e^{N}}$, pois $\log _{2} 2^{e^{N}} \leq \log _{2}\left(e^{e^{N}}+1\right)$. $16\left(\log _{2}\left(e^{N}+1\right)\right)^{2} \leq \log _{2} 2^{e^{N}} \Longleftrightarrow 16\left(\log _{2}\left(e^{N}+1\right)\right)^{2} \leq e^{N} \Longleftrightarrow \log _{2}\left(e^{N}+1\right) \leq \frac{e^{\frac{N}{2}}}{4}$.

Como $\log _{2}\left(e^{N}+1\right) \leq \log _{2} 4^{N}=2 N$, basta demonstrar que $2 N \leq \frac{e^{\frac{N}{2}}}{4}$, ou seja, $8 N \leq e^{\frac{N}{2}}$, mas já vimos anteriormente que $8 x \leq e^{\frac{x}{2}}$ para $x \geq 14$.

Portanto $8 N \leq e^{\frac{N}{2}}$ e assim temos o resultado $\frac{x}{f(x)^{1 / 2}} \leq \frac{x}{4 f\left(x_{0}\right)}$

Lema 4.13 Sejam $N \in L, n \in[\ln N, \exp N], 0<\epsilon \leq 1 / 2$ e $x_{1}, \ldots, x_{n}$ uma RIS com constante $1+\epsilon$. Então $\left\|\sum_{i=1}^{n} x_{i}\right\| \leq\left(1+\epsilon+\epsilon^{\prime}\right) n f(n)^{-1}$.

\section{Demonstração:}

Pela definição implicita do espaço normado $X$ temos que ele satisfaz uma $f$-estimativa inferior. (demonstração análoga a do espaço de Thomas Schlumprecht)

Seja $g$ a função definida na Observação 2.10 considerando $K_{0}=K$. Como $g \leq \phi$ temos

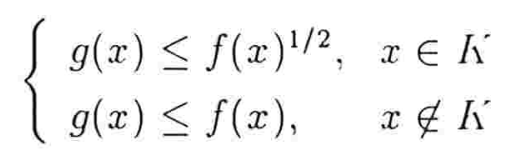

Sabemos também que $f(x)^{1 / 2} \leq g(x) \operatorname{logo} g(x)=f(x)^{1 / 2}$ quando $x \in K$.

Consideremos $k \in K, \bar{g} \in B_{k}^{*}(X) \operatorname{logo} \bar{g}=f(k)^{-1 / 2} \sum_{i=1}^{k} g_{i}$, onde $\left(g_{1}, \ldots, g_{k}\right) \in \Gamma_{k}^{X}$.

Como $k \in K$ temos que $f(k)^{-1 / 2}=g(k)^{-1} \operatorname{logo} \bar{g}=\sum_{i=1}^{k} g(k)^{-1} g_{i}$. Pela Observação 4.5 temos que $\left\|g_{i}\right\| \leq 1$, para qualquer $i, 1 \leq i \leq k$. Seja $x \in X$ com $\|x\| \leq 1$.

Pela definição de norma temos $|\bar{g}(E x)| \leq\|x\|, E \subset \mathbb{N}$. Tomando $E=\operatorname{ran}(x)$ segue $|\bar{g}(x)| \leq\|x\|, \log 0\|\bar{g}\| \leq 1, \forall x \in X$.

Portanto $\bar{g} \in B_{k}^{*}(X)$ quando $k \in K^{\prime}$ é uma $(k, g)$-forma $(k \geq 2)$.

Verificaremos agora que dado $x \in X, E x$ é normado por uma (M,g)-forma ou tem a norma do supremo. 
De fato, pela definição de norma temos 1) $\|E x\|=\|E x\|_{\infty} \quad$ ou $\quad$ 2) $\|E x\|=$ $\sup \left\{|\bar{g}(\bar{E} E x)|: k \in K^{\prime}, \bar{g} \in B_{k}^{*}(X), \bar{E} \subset \mathbb{N}\right\} \quad$ ou $\left.\quad 3\right)\|E x\|=\sup \left\{f(M)^{-1} \sum_{i=1}^{M}\left\|E_{i} E x\right\|\right.$ : $\left.2 \leq M \in \mathbb{N}, E_{1}<\ldots<E_{M}\right\}$.

Em 2) temos que como $\bar{g} \in B_{k}^{*}(X)$ com $k \in K$ é uma (k, g)-forma, $k \geq 2$ o $\sup \left\{|\bar{g}(\bar{E} E x)|: k \in K^{\prime}, \bar{g} \in B_{k}^{*}(X), \bar{E} \subset \mathbb{N}\right\} \leq \sup \left\{\left|x^{*}(\bar{E} E x)\right|: M \geq 2, x^{*}\right.$ é uma (M,g)forma $\}=\sup \left\{\left|\left(\bar{E} x^{*}\right)(E x)\right|: M \geq 2, x^{*}\right.$ é uma (M,g)-forma $\}$. Como $x^{*}$ é uma (M,g)forma temos que $\bar{E} x^{*}$ também é uma (M,g)-forma, logo $\|E x\| \leq \sup \left\{\left|x^{*}(E x)\right|: M \geq 2, x^{*}\right.$ é uma $(\mathrm{M}, \mathrm{g})$-forma $\}$.

Em 3) temos $\|E x\|-\epsilon<f(M)^{-1} \sum_{i=1}^{M}\left\|E_{i} E x\right\|$. Consideremos $\left(x_{i}^{*}\right)_{i=1}^{M}$ os funcionais suporte de $\left(E_{i} E x\right)_{i=1}^{M}$ e $x^{*}=f(M)^{-1} \sum_{i=1}^{M} x_{i}^{*}$, os funcionais foram definidos como no Corolário 3.17 e como já verificamos neste Corolário $x^{*}$ é uma (M,f)-forma. Logo $\left\|x^{*}\right\| \leq 1$, $\left\|f(M)^{-1} x_{i}^{*}\right\|=f(M)^{-1}\left\|x_{i}^{*}\right\| \leq f(M)^{-1} \leq g(M)^{-1}, \forall i$ e $x_{1}^{*}<\ldots<x_{M}^{*}$. Portanto $x^{*}$ é uma (M,g)-forma, $M \geq 2$.

Então $x^{*}(E x)=f(M)^{-1} \sum_{i=1}^{M} x_{i}^{*}(E x)=f(M)^{-1} \sum_{i=1}^{M}\left\|E_{i} E x\right\|>\|E x\|-\epsilon$. Portanto $\|E x\|-\epsilon<x^{*}(E x) \leq\left|x^{*}(E x)\right|$, onde $x^{*}$ é uma (M,g)-forma, $M \geq 2$. Fazendo $\epsilon \rightarrow 0$ temos $\|E x\| \leq\left|x^{*}(E x)\right|$.

Portanto $\|E x\| \leq \sup \left\{\left|x^{*}(E x)\right|: M \geq 2, x^{*}\right.$ é uma (M,g)-forma $\}$.

Consideremos $x=\sum_{i=1}^{n} x_{i}$ e $E$ um intervalo tal que $\lambda(E) \geq 1$. Como $\lambda(E) \geq 1$, temos pelo Lema 3.20 que $\|E x\| \geq \frac{1}{4}$ e análogo ao que fizemos, na página 77 , para o espaço de Schlumprecht temos $\|E x\|_{\infty}<\frac{1}{4}$.

Portanto não ocorre a norma do supremo para intervalos $\operatorname{com} \lambda(E) \geq 1$.

Como $g \in \mathcal{F},\|E x\| \leq \sup \left\{\left|x^{*}(E x)\right|: M \geq 2, x^{*}\right.$ é uma (M,g)-forma $\}$ e $g \geq f^{1 / 2}$ todas as hipóteses do Lema 3.21 estão satisfeitas. Portanto $\left\|\sum_{i=1}^{n} x_{i}\right\| \leq\left(1+\epsilon+\epsilon^{\prime}\right) n g(n)^{-1}=$ $\left(1+\epsilon+\epsilon^{\prime}\right) n \frac{G(n)}{n}=\left(1+\epsilon+\epsilon^{\prime}\right) G(n)$. 
Pelo Lema 4.12 temos $G(n)=n f(n)^{-1}$, logo $\left\|\sum_{i=1}^{n} x_{i}\right\| \leq\left(1+\epsilon+\epsilon^{\prime}\right) n f(n)^{-1}$.

Lema 4.14 Sejam $N \in L, 0<\epsilon<1 / 4, M=N^{\epsilon}$ e $x_{1}, \ldots, x_{N}$ uma RIS com constante $1+\epsilon$. Então $\sum_{i=1}^{N} x_{i}$ é um $l_{1+}^{M}$-vetor com constante $(1+4 \epsilon)$.

\section{Demonstração:}

Sejam $m=\frac{N}{M}=N^{1-\epsilon}, x=\sum_{i=1}^{N} x_{i}$ e para $j, 1 \leq j \leq M, y_{j}=\sum_{i=(j-1) m+1}^{j m} x_{i}$ então $y_{1}=\sum_{i=1}^{m} x_{i}, x_{1}<\ldots<x_{m} . y_{1}$ é a soma de uma RIS de comprimento $m$ com constante $(1+\epsilon)$.

$y_{j}=\sum_{i=(j-1) m+1}^{j m} x_{i}, j m-[(j-1) m+1]+1=j m-j m+m-1+1=m$. Portanto $y_{j}$ é a soma de uma RIS de comprimento $m$ com constante $(1+\epsilon)$.

Vamos usar o Lema 4.13, mas para isto precisamos verificar que $m \in[\ln N, \exp N]$, ou seja, que $\ln N \leq m \leq \exp N$. Temos $0<\epsilon<1 / 4$ então $3 / 4<1-\epsilon<1, m=N^{1-\epsilon}$.

1) $x^{1-\epsilon} \leq e^{x}$. Seja $d(x)=e^{x}-x^{1-\epsilon}$, vamos provar que $d(x) \geq 0$.

$d(0)=1, d^{\prime}(x)=e^{x}-(1-\epsilon) x^{-\epsilon}=e^{x}-\frac{(1-\epsilon)}{x^{\epsilon}}$.

$e^{x}-\frac{(1-\epsilon)}{x^{\epsilon}}>0 \Longleftrightarrow e^{x}>\frac{(1-\epsilon)}{x^{\epsilon}} \Longleftrightarrow \ln e^{x}>\ln \frac{(1-\epsilon)}{x^{\epsilon}} \Longleftrightarrow x \ln e>\ln (1-\epsilon)-\ln x^{\epsilon} \Longleftrightarrow$ $\underbrace{x}_{>0}>\underbrace{\ln (1-\epsilon)}_{<0}-\underbrace{\epsilon \ln x}_{>0}$ o que é verdade pois um número positivo é maior que um número negativo.

Portanto $d(x) \geq 0$. Logo $x^{1-\epsilon} \leq e^{x}$.

2) $x^{1-\epsilon} \geq \ln x$. Seja $d(x)=x^{1-\epsilon}-\ln x$, vamos provar que $d(x) \geq 0$.

$d(1)=1, d^{\prime}(x)=(1-\epsilon) x^{-\epsilon}-\frac{1}{x}=\frac{(1-\epsilon)}{x^{\epsilon}}-\frac{1}{x}$.

$\frac{(1-\epsilon)}{x^{\epsilon}}-\frac{1}{x}>0 \Longleftrightarrow \frac{x^{1-\epsilon}(1-\epsilon)-1}{x}>0 \Longleftrightarrow \frac{1}{x}\left(x^{1-\epsilon}(1-\epsilon)-1\right)>0$.

Seja $H(x)=x^{1-\epsilon}(1-\epsilon)-1$.

$H(16)=(16)^{1-\epsilon}(1-\epsilon)-1$. Temos que $(16)^{1-\epsilon}(1-\epsilon)>(16)^{3 / 4} \frac{3}{4}=6>0$.

$H^{\prime}(x)=(1-\epsilon)^{2} x^{-\epsilon}=\frac{(1-\epsilon)^{2}}{x^{\epsilon}}>0$. Portanto $H$ é positiva. $\log 0 \frac{(1-\epsilon)}{x^{\epsilon}}-\frac{1}{x}>0$.

Portanto $d(x) \geq 0$. Logo $x^{1-\epsilon} \geq \ln x$. 
Então pelo Lema $4.13\left\|y_{j}\right\| \leq\left(1+\epsilon+\epsilon^{\prime}\right) m f(m)^{-1}=(1+2 \epsilon) m f(m)^{-1}$ para qualquer $j$. Verificaremos agora que $x$ é um $l_{1+}^{M}$-vetor.

$\sum_{i=1}^{M} y_{i}=\sum_{i=1}^{M} \sum_{j=(i-1) m+1}^{i m} x_{j}=\sum_{j=1}^{m} x_{i}+\sum_{j=m+1}^{2 m} x_{i}+\sum_{j=2 m+1}^{3 m} x_{i}+\ldots+\sum_{j=(M-1) m+1}^{M m} x_{i}=\sum_{i=1}^{N} x_{i}=x$.

Portanto $x=\sum_{i=1}^{M} y_{i} . X$ satisfaz uma $f$-estimativa inferior, então consideremos $E_{i}=$ $\operatorname{ran}\left(x_{i}\right)$.

$\|x\| \geq f(N)^{-1} \sum_{i=1}^{N}\left\|E_{i} x\right\|=f(N)^{-1} \sum_{i=1}^{N}\left\|E_{i}\left(x_{1}+\ldots+x_{N}\right)\right\|=f(N)^{-1}\left(\left\|E_{1} x_{1}\right\|+\ldots+\right.$ $\left.\left\|E_{N}\left(x_{N}\right)\right\|\right)=f(N)^{-1}\left(\left\|x_{1}\right\|+\ldots+\left\|x_{N}\right\|\right)=f(N)^{-1} N$, pois $\left\|x_{i}\right\|=1, \forall i$.

Ora $\left\|y_{i}\right\| \leq \frac{(1+2 \epsilon) m}{f(m)}=\frac{(1+2 \epsilon) \frac{N}{M}}{f(m)}=\frac{(1+2 \epsilon) N}{M f(m)}=\frac{(1+2 \epsilon)}{f(m) M} N=\frac{(1+2 \epsilon) f(N)}{f(m) M} \frac{N}{f(N)}=\frac{\frac{(1+2 \epsilon) f(N)}{f(m)}}{M} \frac{N}{f(N)}$.

Portanto $\left\|y_{i}\right\| \leq \frac{\frac{(1+2 e) f(N)}{f(m)}}{M} \frac{N}{f(N)} \leq \frac{\frac{(1+2 e) f(N)}{f(m)}}{M}\|x\|$.

Logo $x$ é um $l_{1+}^{M}$ vetor com constante $C=\frac{(1+2 \epsilon) f(N)}{f(m)}$.

Temos que $m=N^{1-\epsilon}$, então vamos provar que $\frac{f(N)}{f(m)} \leq(1-\epsilon)^{-1}$.

$\frac{f(N)}{f(m)} \leq(1-\epsilon)^{-1} \Longleftrightarrow f(N)(1-\epsilon) \leq f(m) \Longleftrightarrow(1-\epsilon) \log _{2}(N+1) \leq \log _{2}\left(N^{1-\epsilon}+1\right) \Longleftrightarrow$ $\log _{2}(N+1)^{1-\epsilon} \leq \log _{2}\left(N^{1-\epsilon}+1\right) \Longleftrightarrow(N+1)^{1-\epsilon} \leq N^{1-\epsilon}+1 \Longleftrightarrow(N+1)^{1-\epsilon}-N^{1-\epsilon} \leq 1$.

Consideremos $h(x)=(N+x)^{1-\epsilon}$, então $h^{\prime}(x)=(1-\epsilon)(N+x)^{-\epsilon}$.

Pelo Teorema do Valor Médio temos que $\frac{h(1)-h(0)}{1-0}=h^{\prime}(c)$, onde $0<c<1$.

Sabemos que $h(1)=(N+1)^{1-\epsilon}$ e $h(0)=(N)^{1-\epsilon}$ então segue que $(N+1)^{1-\epsilon}-(N)^{1-\epsilon}=$ $(1-\epsilon)(N+c)^{-\epsilon}=(1-\epsilon)\left(\frac{1}{N+c}\right)^{\epsilon} \leq 1$. $1+4 \epsilon$.

Sendo assim temos que $C=\frac{(1+2 \epsilon) f(N)}{f(m)} \leq \frac{(1+2 \epsilon)}{(1+\epsilon)}$. Vamos verificar agora que $\frac{(1+2 \epsilon)}{(1+\epsilon)} \leq$ $\frac{(1+2 \epsilon)}{(1+\epsilon)} \leq 1+4 \epsilon \Longleftrightarrow(1+2 \epsilon) \leq(1+\epsilon)(1+4 \epsilon)$ o que é verdade pois $(1+\epsilon)(1+4 \epsilon)>$ $>(1+4 \epsilon)>(1+2 \epsilon)$. Portanto $x$ é um $l_{1+}^{M}$-vetor com constante $(1+4 \epsilon)$.

Lema 4.15 Sejam $k \in K$ e $x_{1}^{*}, \ldots, x_{k}^{*}$ uma sequência especial de comprimento $k$, onde cada $x_{i}^{*}$ é uma $\left(M_{i}, f\right)$-forma. Seja $x_{1}, \ldots, x_{k}$ uma sequência de vetores sucessivos tais que todo $x_{i}$ é um vetor RIS normalizado de comprimento $M_{i}$ e constante $1+\epsilon / 4, \epsilon=$ $1 / 10$ e ran $\left(x_{i}\right)=\operatorname{ran}\left(x_{i}^{*}\right)$. Assuma que $\left|\left(\sum_{i=1}^{k} x_{i}^{*}\right)\left(\sum_{i=1}^{k} E x_{i}\right)\right| \leq 2$ para todo intervalo $E$. 
Então $\left\|\sum_{i=1}^{k} x_{i}\right\| \leq(1+2 \epsilon) k / f(k)$.

\section{Demonstração:}

Seja $\mu=1 / 40$. Temos que $x_{i}$ é um vetor RIS normalizado, logo $x_{i}=\frac{x_{i}^{\prime}}{\left\|x_{i}^{\prime}\right\|}$ onde $x_{i}^{\prime}=x_{i 1}+\ldots+x_{i M_{i}}$ é a soma de uma RIS com constante $(1+\mu)$. Coloquemos $\alpha_{i}=\frac{1}{\left\|x_{i}^{\prime}\right\|}$.

Pelo Lema $4.14 \frac{x_{i}}{\alpha_{i}}$ é um $l_{1+}^{N_{i}}$ vetor com constante $(1+4 \mu)=(1+\epsilon)$ onde $N_{i}=M_{i}^{\epsilon / 4}$. Logo $\frac{x_{i}}{\alpha_{i}}=\sum_{j=1}^{N_{i}} y_{j}$ onde $\left\|y_{j}\right\| \leq \frac{(1+\epsilon)}{N_{i}}\left\|\frac{x_{i}}{\alpha_{i}}\right\|$, ou seja, $\left\|\alpha_{i} y_{j}\right\| \leq \frac{(1+\epsilon)}{N_{i}}$.

Então $x_{i}=\sum_{j=1}^{N_{i}} \alpha_{i} y_{j},\left\|x_{i}\right\|=1$ e $\left\|\alpha_{i} y_{j}\right\| \leq \frac{(1+\epsilon)}{N_{i}}$, portanto por definição $x_{i}$ é um $l_{1+}^{N_{i}}$ average com constante $(1+\epsilon)$, onde $N_{i}=M_{i}^{\epsilon / 4}$.

Temos que $M_{1}=j_{2 k}$, pois cada $x_{i}^{*}$ é uma $\left(M_{i}, f\right)$-forma e $x_{1}^{*}, \ldots, x_{k}^{*}$ é uma sequência especial, logo $x_{1}^{*} \in A_{j_{2 k}}^{*}$ e $x_{i+1}^{*} \in A_{\sigma\left(x_{i}^{*}, \ldots, x_{i}^{*}\right)}^{*}$ para qualquer $i, 1 \leq i \leq k-1$. Sabemos pelo Lema 4.2 que $M_{1}^{\epsilon / 4}=N_{1} \geq 4 M_{f}(k / \epsilon) / \epsilon f^{\prime}(1)$.

Lembremos que $\sigma$ foi escolhida de tal forma que se $z_{1} \ldots, z_{s}$ é uma sequência de vetores sucessivos em $Q, S=\sigma\left(z_{1}, \ldots, z_{s}\right)$ e $z=\sum_{i=1}^{s} z_{i}$, então $\frac{1}{20} f\left(S^{1 / 40}\right)^{1 / 2} \geq|\operatorname{ran}(z)|$. Logo

1) $N_{1} \geq 4 M_{f}(k / \epsilon) / \epsilon f^{\prime}(1)>2(1+\epsilon) M_{f}(k / \epsilon) / \epsilon f^{\prime}(1)$.

2) cada $x_{i}$ é um $l_{1+}^{N_{i}}$-average com constante $(1+\epsilon), x_{1}<\ldots<x_{k}$.

3) $\frac{1}{20} f\left(S^{1 / 40}\right)^{1 / 2} \geq|\operatorname{ran}(z)| \operatorname{logo} \frac{\epsilon}{2} f\left(S^{1 / 40}\right)^{1 / 2} \geq|\operatorname{ran}(z)|$.

$S^{1 / 40}=S^{\epsilon / 4}=M_{i}^{\epsilon / 4}=N_{i}$, pois $M_{i}=\sigma\left(x_{1}^{*}, \ldots, x_{i-1}^{*}\right)$ onde $x_{1}^{*}, \ldots, x_{k}^{*}$ é uma sequência especial.

$|\operatorname{ran}(z)|=\left|\operatorname{ran}\left(z_{1}+\ldots+z_{s}\right)\right| \geq\left|\operatorname{ran}\left(z_{i}\right)\right|$, para qualquer $i, 1 \leq i \leq s$.

Portanto $\frac{\epsilon}{2} f\left(N_{i}\right)^{1 / 2} \geq\left|\operatorname{ran}\left(x_{i-1}\right)\right|, i=2, \ldots, k$.

Por 1$), 2)$ e 3 ) segue que $x_{1}, \ldots, x_{k}$ é uma RIS de comprimento $k$ com constante $(1+\epsilon)$.

Para provar o Lema vamos usar o Lema 3.21. Mas primeiro mostraremos que se $z_{1}^{*}, \ldots, z_{k}^{*}$ é uma sequência especial de funcionais de comprimento $k$ e $E$ é qualquer intervalo, 
então $\left|z^{*}(E x)\right| \leq 1 / 4$, onde $z^{*}$ é a $(k, \sqrt{f})$-forma $f(k)^{-1 / 2} \sum_{i=1}^{k} z_{i}^{*}$ e $x=\sum_{i=1}^{k} x_{i}$.

Seja $t$ o maior índice tal que $z_{t}^{*}=x_{t}^{*}$ ou zero se $t$ não existir. Suponha que $i \neq j$ ou que $i$ ou $j$ é maior do que $t+1$. Então, como $\sigma$ é injetora podemos achar $L_{1} \neq L_{2} \in L$ tal que $z_{i}^{*}$ é uma $\left(L_{1}, f\right)$-forma e $x_{j}$ é a soma de uma RIS normalizada de comprimento $L_{2}$ e também um $l_{1+}^{L_{2}^{\prime}}$-average com constante $(1+\epsilon)$, onde $L_{2}^{\prime}=L_{2}^{\epsilon / 4}$. Como no fim do capítulo 3 nós usaremos os Lemas 3.13 e 3.16 para mostrar que $\left|z_{i}^{*}\left(E x_{j}\right)\right|<k^{-2}$.

Se $L_{1}<L_{2}$ segue do Lema 4.2 que $4 L_{1}^{2} \leq \ln \ln \ln L_{2}$, logo $L_{2} \geq e^{4 L_{1}^{2}}$ consequentemente $L_{2}^{1 / 40} \geq e^{\frac{4 L_{1}^{2}}{40}}=e^{\frac{L_{1}^{2}}{10}} \geq \frac{L_{1}^{2}}{10}=\underbrace{\frac{L_{1}}{10}}_{>1} L_{1}>L_{1}$. Portanto se $L_{1}<L_{2}$ temos $L_{1}<L_{2}^{\prime}$.

Sabemos que $L_{1} \geq j_{2 k}$, visto que $L_{1}$ aparece em uma sequência especial de comprimento $k$. Assim temos, $\left|z_{i}^{*}\left(E x_{j}\right)\right| \leq\left|z_{i}^{*}\left(x_{j}\right)\right|=\left|\sum_{k=1}^{L_{1}} y_{k}^{*}\left(x_{j}\right)\right| \leq \sum_{k=1}^{L_{1}}\left|y_{k}^{*}\left(x_{j}\right)\right|$, onde $z_{i}^{*}=\sum_{k=1}^{L_{1}} y_{k}^{*}$. Considerando $E_{k}=\operatorname{ran}\left(y_{k}^{*}\right)$ temos $\sum_{k=1}^{L_{1}}\left|y_{k}^{*}\left(x_{j}\right)\right|=\sum_{k=1}^{L_{1}}\left|E_{k}\left(y_{k}^{*}\left(x_{j}\right)\right)\right|=\sum_{k=1}^{L_{1}}\left|y_{k}^{*}\left(E_{k} x_{j}\right)\right| \leq$ $\sum_{k=1}^{L_{1}}\left\|y_{k}^{*}\right\|\left\|\left(E_{k} x_{j}\right)\right\| \leq f\left(L_{1}\right)^{-1} \sum_{k=1}^{L_{1}}\left\|E_{k} x_{j}\right\| \leq f\left(L_{1}\right)^{-1}(1+\epsilon)\left(1+2 L_{1} / L_{2}^{\prime}\right) . \quad L_{1}<L_{2}^{\prime} \operatorname{logo}$ $L_{1} / L_{2}^{\prime}<1$. portanto $\left|z_{i}^{*}\left(E x_{j}\right)\right| \leq \frac{3(1+\epsilon)}{f\left(L_{1}\right)}$.

A conclusão deste caso segue do fato de que $f(l) \geq 4 k^{2}$ quando $l \geq j_{2 k}$.

Observação: De $\ln x<x \leq x+1$ segue que $\ln \ln x \leq \ln (x+1)$. Mas $\ln (x+1)=\frac{\log _{2} x+1}{\log _{2} e}$, $\operatorname{logo} \underbrace{\ln \ln x}_{A} \leq \frac{\log _{2} x+1}{\log _{2} e}$ então $\log _{2} e \ln \ln x \leq \log _{2}(x+1)$.

Mas como $\ln A \leq A \leq \log _{2} e A$ segue que $\ln \ln \ln x \leq \log _{2} e \ln \ln x \leq \log _{2}(x+1)$.

Vamos provar que se $j_{2 k} \leq l$ então $f(l) \geq 4 k^{2}$.

Sabemos que $4 k^{2} \leq 4.4 k^{2} \leq 4\left(j_{2 k}\right)^{2} \leq \ln \ln \ln l \leq f(l)$.

Portanto, $\left|z_{i}^{*}\left(E x_{j}\right)\right| \leq \frac{3(1+\epsilon)}{f\left(L_{1}\right)} \leq \frac{3(1+\epsilon)}{4 k^{2}}<\frac{4}{4 k^{2}}=\frac{1}{k^{2}}=k^{-2}$. Logo $\left|z_{i}^{*}\left(E x_{j}\right)\right|<k^{-2}$ se $L_{1}<L_{2}$.

Se $L_{2}<L_{1}$ aplicaremos o Lema 3.16 para $x_{j}^{\prime}$, onde $x_{j}^{\prime}$ é a soma da RIS que originou $x_{j}$, com $\epsilon_{1}=1$. mas primeiro temos que verificar que $M_{f}\left(L_{2}\right)<L_{1}$. 
Como $L_{2}<L_{1}$ segue do Lema 4.2 que $\ln \ln \ln L_{1} \geq 4 L_{2}^{2}$, $\log L_{1} \geq e^{e^{e^{4 L_{2}^{2}}}}$. Como $L_{1} \geq e^{e^{e^{4 L_{2}^{2}}}}$ temos que $f\left(L_{1}\right) \geq f\left(e^{e^{e^{4 L_{2}^{2}}}}\right)$. Vamos verificar agora que $f\left(e^{e^{e^{4} L_{2}^{2}}}\right)>36 L_{2}^{2}$.

$f\left(e^{e^{e^{4 L_{2}^{2}}}}\right)>36 L_{2}^{2} \Longleftrightarrow \log _{2}\left(e^{e^{e^{4 L_{2}^{2}}}}+1\right)>36 L_{2}^{2} \Longleftrightarrow 2^{36 L_{2}^{2}}=\left(2^{9}\right)^{4 L_{2}^{2}}<e^{e^{e^{4 L_{2}^{2}}}}$ o que é verdade pois $e^{e^{e}}>2^{9}=512$. Então temos $f\left(L_{1}\right) \geq f\left(e^{e^{e^{4} L_{2}^{2}}}\right)>36 L_{2}^{2}$.

$M_{f}\left(L_{2}\right)=f^{-1}\left(36 L_{2}^{2}\right), M_{f}\left(L_{2}\right)<L_{1} \Longleftrightarrow f\left(M_{f}\left(L_{2}\right)\right)<f\left(L_{1}\right) \Longleftrightarrow 36 L_{2}^{2}<f\left(L_{1}\right)$ o que já vimos acima que é verdade. Portanto temos que se $L_{2}<L_{1}$ então $M_{f}\left(L_{2}\right)<L_{1}$.

Vamos aplicar o Lema 3.16 para $x_{j}^{\prime} \operatorname{com} f=g, \epsilon_{1}=1$. Podemos aplicar este Lema porque $f \geq f^{1 / 2}, X$ satisfaz uma $f$-estimativa inferior, $\epsilon_{1}>0, L_{1}>M_{f}\left(L_{2}\right)$ e $z_{i}^{*}$ é uma $\left(L_{1}, f\right)$-forma. Portanto $\left|z_{i}^{*}\left(E x_{j}^{\prime}\right)\right| \leq\left(1+\epsilon_{1}+\epsilon_{1}^{\prime}\right)=3$.

$X$ satisfaz uma $f$-estimativa inferior $\log \left\|x_{j}^{\prime}\right\| \geq f\left(L_{2}\right)^{-1} \sum_{i=1}^{L_{2}}\left\|E_{i} x_{j}^{\prime}\right\|$. Considere $E_{i}=$ $\operatorname{ran}\left(x_{j i}\right), i=1,2, \ldots, L_{2}$. Então $\left\|x_{j}^{\prime}\right\| \geq f\left(L_{2}\right)^{-1} L_{2}$.

Quando $l \geq j_{2 k}$ temos que $\frac{f(l)}{l} \leq \frac{1}{4 k^{2}}$, logo $\frac{L_{2}}{f\left(L_{2}\right)} \geq 4 k^{2}$.

De fato, temos $j_{2 k} \leq l$ e sabemos que a função $\frac{x}{f(x)}$ é não decrescente, então $\frac{j_{2 k}}{f\left(j_{2 k}\right)} \leq \frac{l}{f(l)}$ $\log \frac{f(l)}{l} \leq \frac{f\left(j_{2 k}\right)}{j_{2 k}}$. Usando derivada é fácil verificar que $\log _{2}(x+1) \leq \sqrt{x}$.

Sendo assim temos $\frac{f(l)}{l} \leq \frac{f\left(j_{2 k}\right)}{j_{2 k}} \leq \frac{\sqrt{j_{2 k}}}{j_{2 k}}=\frac{1}{\sqrt{j_{2 k}}}$. Pelo ítem 7 do Lema 4.2 temos $\sqrt{J_{2 k}} \geq 4 k^{2} \log 0 \frac{1}{\sqrt{32 k}} \leq \frac{1}{4 k^{2}}$.

Então $\left|z_{i}^{*}\left(E \frac{x_{j}^{\prime}}{\left\|x^{\prime},\right\|}\right)\right|=\frac{1}{\left\|x_{j}^{\prime}\right\|}\left|z_{i}^{*}\left(E x_{j}^{\prime}\right)\right| \leq \frac{3}{\left\|x_{j}^{\prime}\right\|} \leq \frac{3}{4 k^{2}}<\frac{1}{k^{2}}=k^{-2}$.

Portanto $\left|z_{i}^{*}\left(E x_{j}\right)\right|<k^{-2}$ se $L_{2}<L_{1}$.

Agora consideremos $F$ um intervalo tal que $\left|\left(\sum_{i=1}^{t} z_{i}^{*}\right)(E x)\right|=\left|\left(\sum_{i=1}^{k} x_{i}^{*}\right)(F x)\right| \leq 2$.

( Basta pegar $F$ igual ao intervalo $\left\{j_{1}, \ldots, j_{m}\right\}$ com $j_{1}$ sendo o minimo de $E$ tal que $\operatorname{ran}\left(z_{i}^{*}\right) \cap E \neq \emptyset$ e $j_{m}$ sendo o máximo de $E$ tal que $\operatorname{ran}\left(z_{j}^{*}\right) \cap E \neq \emptyset$, onde $i$ e $j$ são respectivamente o menor e o maior valor tal que $E z_{i}^{*}$ e $E z_{j}^{*}$ são não nulos, $1 \leq i, j \leq t$.)

$$
\begin{aligned}
& \text { Segue que }\left|\left(\sum_{i=1}^{k} z_{i}^{*}\right)(E x)\right| \leq\left|\left(\sum_{i=1}^{t} z_{i}^{*}\right)(E x)\right|+\left|z_{t+1}^{*}(E x)\right|+\left|\left(\sum_{i=t+2}^{k} z_{i}^{*}\right)(E x)\right| \leq \\
& \leq 2+\left|z_{t+1}^{*}\left(\sum_{i=1}^{k} E x_{i}\right)\right|+\left|\left(\sum_{i=t+2}^{k} z_{i}^{*}\right)\left(\sum_{j=1}^{k} E x_{j}\right)\right| .
\end{aligned}
$$

Calculando cada módulo da ultima desigualdade separadamente temos, 
1) $\left|z_{t+1}^{*}\left(\sum_{i=1}^{k} E x_{i}\right)\right| \leq \sum_{i=1}^{k}\left|z_{t+1}^{*}\left(E x_{i}\right)\right| \leq\left|z_{t+1}^{*}\left(E x_{t+1}\right)\right|+(k-1) k^{-2}$.

2) $\left|\sum_{i=t+2}^{k} z_{i}^{*}\left(\sum_{j=1}^{k} E x_{j}\right)\right|=\left|\sum_{i=t+2}^{k} \sum_{j=1}^{k} z_{i}^{*}\left(E x_{j}\right)\right| \leq \sum_{i=t+2}^{k} \sum_{j=1}^{k}\left|z_{i}^{*}\left(E x_{j}\right)\right| \leq(k-t-2+1)\left(k k^{-2}\right)=$ $(k-t-1)\left(k k^{-2}\right)$.

Juntando tudo ficamos com,

$\left|\left(\sum_{i=1}^{k} z_{i}^{*}\right)(E x)\right| \leq 2+\left|z_{t+1}^{*}\left(E x_{t+1}\right)\right|+(k-1) k^{-2}+k^{-2}\left(k^{2}-k t-k\right)=2+\left|z_{t+1}^{*}\left(E x_{t+1}\right)\right|+$ $k^{-2}\left(k^{2}-k t-1\right) \leq 2+\left|z_{t+1}^{*}\left(E x_{t+1}\right)\right|+k^{-2} k^{2} \leq 2+\underbrace{\left\|z_{t+1}^{*}\right\|}_{\leq 1} \underbrace{\left\|\left(E x_{t+1}\right)\right\|}_{\leq 1}+1 \leq 4$.

Finalmente obtemos que $\left|z^{*}(E x)\right|=\left|\left(f(k)^{-1 / 2} \sum_{i=1}^{k} z_{i}^{*}\right)(E x)\right|=f(k)^{-1 / 2}\left|\left(\sum_{i=1}^{k} z_{i}^{*}\right)(E x)\right| \leq$ $f(k)^{-1 / 2} 4=\frac{4}{\sqrt{f(k)}}<\frac{4}{16}=\frac{1}{4}$, já que $\sqrt{f\left(j_{1}\right)}>16$.

Agora seja $\phi^{\prime}$ a função

$$
\begin{cases}\left(\log _{2}(y+1)\right)^{1 / 2}, & y \in K, y \neq k \\ \log _{2}(y+1), & y \notin K, y=k\end{cases}
$$

Seja $g^{\prime}$ a função obtida de $\phi^{\prime}$ pela construção explicada na Observação 2.10 no caso $K_{0}=K \backslash\{k\}$.

Pelo Lema 4.12 temos que $G(y)=y f(y)^{-1}$ para todo $y$ no intervalo $[\ln l$, expl] onde $l \in L \cup\{k\}$. Assim temos $g^{\prime}(l)=\frac{l}{G(l)}=\frac{l}{\frac{l}{f(l)}}=f(l)$ para todo $l \in L \cup\{k\}$.

Segue do que mostramos sobre sequências especiais de comprimento $k$ que $\frac{1}{4}<$ $\|E x\| \leq \sup \left\{\left|x^{*}(E x)\right|: M \geq 2, x^{*}\right.$ é uma $\left(M, g^{\prime}\right)-$ forma $\}$ quando $E$ é um intervalo de comprimento maior ou igual a 1 .

Como $x$ é a soma de uma RIS, temos pelo Lema 3.21 que $\|x\| \leq\left(1+\epsilon+\epsilon^{\prime}\right) k g^{\prime}(k)^{-1}=$ $(1+2 \epsilon) k g^{\prime}(k)^{-1}=(1+2 \epsilon) k f(k)^{-1}$.

Lema 4.16 Sejam $z^{*}$ uma $(M, f)$-forma, $M \in L, k \in K, x$ um vetor RIS normalizado de comprimento $M$ satisfazendo $\operatorname{ran}\left(z^{*}\right) \subset \operatorname{ran}(x)$ e $z^{*}(x)=\frac{1}{2}$. Então existe uma $(M, f)$ forma $x^{*} \in Q$ tal que $\left|x^{*}(x)-1 / 2\right| \leq 1 / k \operatorname{com} \operatorname{ran}\left(x^{*}\right)=\operatorname{ran}(x)$. 


\section{Demonstração}

Temos por hipótese que $z^{*}=\sum_{i=1}^{M} z_{i}^{*}$, onde $\left\|z^{*}\right\| \leq 1,\left\|z_{i}^{*}\right\| \leq f(M)^{-1}$ e $z_{1}^{*}<\ldots<z_{M}^{*}$; $x=\sum_{i=1}^{M} x_{i}, x_{1}<\ldots<x_{M}$ e $\operatorname{ran}\left(z_{i}^{*}\right) \subset \operatorname{ran}\left(x_{i}\right)$.

Sejam $n_{i}=\max \operatorname{ran}\left(x_{i}\right), i=1, \ldots, M, N=\max _{1 \leq i \leq M} n_{i}$ e coloquemos $z_{i}^{*}=\left(c_{1}^{i}, c_{2}^{i}, \ldots, c_{n_{i}}^{i}\right)$, onde alguns desses $c_{j^{\prime} s}^{i}$ são nulos.

Seja $\epsilon \in \mathbb{R}$, definindo

1. $\alpha=\frac{1}{f(M)}$,

2. $3(\epsilon)=\epsilon N+a$.

3. $\gamma(\epsilon)=\epsilon N M+1$,

4. $\theta(\epsilon)=\left(1-\frac{\alpha}{\epsilon N+\alpha}\right)(\epsilon N M+1)+\epsilon N M$,

5. $\mu(\epsilon)=\frac{\alpha}{\beta(\epsilon) \gamma(\epsilon)}$.

Como $\lim _{\epsilon \rightarrow 0^{+}} \theta(\epsilon)=0, \mu(0)=1, \mu(\epsilon) \leq 1, \forall \epsilon \in \mathbb{R}$ e $\mu$ é contínua, pelo teorema do valor intermediario do cálculo, existe $\epsilon \in \mathbb{R}$ tal que

6. $\theta(\epsilon)<\frac{1}{k} \mathrm{e}$

†. $\mu(\epsilon)$ é um número racional.

Seja $r_{i}^{*}=\left(b_{1}^{i}, b_{2}^{i}, \ldots, b_{n_{i}}^{i}\right) \in Q$ onde os $b_{j^{\prime} s}^{i}$ são não nulos, $i=1, \ldots, M \operatorname{com}\left|c_{j}^{i}-b_{j}^{i}\right| \leq \epsilon$, $j=1, \ldots, n_{i}$.

Seja $w \in X,\|w\| \leq 1$ com $w=\sum_{i=1}^{\infty} a_{i} e_{i}$, temos $\left|r_{i}^{*}(w)-z_{i}^{*}(w)\right|=\left|\sum_{j=1}^{n_{i}} a_{j}\left(c_{j}^{i}-b_{j}^{i}\right)\right| \leq$ $\epsilon n_{i} \max _{1 \leq j \leq n_{i}}\left|a_{j}\right| \leq \epsilon N$. Logo $\left|r_{i}^{*}(w)\right| \leq\left|r_{i}^{*}(w)-z_{i}^{*}(w)\right|+\left|z_{i}^{*}(w)\right| \leq \epsilon N+\frac{1}{f(M)}=\beta(\epsilon)$ e ainda $\left\|\frac{r_{i}^{*}}{\beta(\epsilon)}\right\| \leq 1, \forall i, \frac{\alpha}{\beta(\epsilon)} \leq 1$.

Consideremos, agora, $r^{*}=\sum_{i=1}^{M} r_{i}^{*}$. Seja $w \in X,\|w\| \leq 1$. Logo $\left|r^{*}(w)-z^{*}(w)\right| \leq$ $\sum_{i=1}^{M}\left|r_{i}^{*}(w)-z_{i}^{*}(w)\right| \leq \epsilon$ VM. Portanto $\left|r^{*}(w)\right| \leq\left|r^{*}(w)-z^{*}(w)\right|+\left|z^{*}(w)\right| \leq \epsilon N M+1=\gamma(\epsilon)$ e ainda $\left\|\frac{r^{\circ}}{\gamma(\epsilon)}\right\| \leq 1, \gamma(\epsilon) \geq 1$. 
Vamos definir $x^{*}$ pondo $x^{*}=\frac{\alpha}{\beta(\epsilon) \gamma(\epsilon)} r^{*}, \operatorname{logo} x^{*}=\sum_{i=1}^{M} \frac{\alpha}{\beta(\epsilon) \gamma(\epsilon)} r_{i}^{*}$. Portanto $x^{*}$ tem as seguintes propriedades:

a) $\left\|\frac{\alpha}{\beta(\epsilon) \gamma(\epsilon)} r_{i}^{*}\right\|=\frac{1}{\gamma(\epsilon)}\left\|\frac{a}{\beta(\epsilon)} r_{i}^{*}\right\| \leq \alpha=\frac{1}{f(M)}$.

b) $\left\|x^{*}\right\|=\left\|\frac{a}{\beta(\epsilon) \gamma(\epsilon)} r^{*}\right\| \leq \frac{a}{\beta(\epsilon)}\left\|\frac{1}{\gamma(\epsilon)} r^{*}\right\| \leq 1$.

c) $r_{1}^{*}<\ldots<r_{M}^{*}$ e $\operatorname{ran}\left(x^{*}\right)=\operatorname{ran}(x)$.

d) $\left|x^{*}(x)-\frac{1}{2}\right|=\left|\frac{\alpha}{\beta(\epsilon) \gamma(\epsilon)} r^{*}(x)-z^{*}(x)\right| \leq\left|\frac{a}{\beta(\epsilon) \gamma(\epsilon)} r^{*}(x)-r^{*}(x)\right|+\left|r^{*}(x)-z^{*}(x)\right| \leq$ $\left\|r^{*}(x)\right\|\left(1-\frac{\alpha}{\beta(\epsilon)}\right)+\epsilon N M \leq\left(1-\frac{\alpha}{\beta(\epsilon)}\right)(\epsilon N M+1)+\epsilon N M<\frac{1}{k}$.

Por a), b), c) e d) temos que $x^{*}$ é uma $(M, f)$-forma, $x^{*} \in Q$ e $\left|x^{*}(x)-\frac{1}{2}\right|<\frac{1}{k}$.

Finalmente já estamos em condições de demonstrarmos o seguinte Teorema.

Teorema 4.17 O espaço de Gowers e Maurey é hereditariamente indecomponível.

\section{Demonstração}

Sejam $Y$ e $Z$ dois subespaços fechados de dimensão infinita de $X_{G M}$ tal que $Y \cap Z=$ $\{0\}$. Mostraremos que a projeção de $Y+Z$ para $Y$ dada por $y+z \rightarrow y$ não é contínua, logo $Y+Z$ não é fechado em $X_{G M}$ e então nào teremos $W$ fechado tal que $W=Y \oplus Z$.

Pois se $Y \cap Z=\{0\}$ e $Y+Z$ é fechado em $X_{G M}$ então definindo $P: Y \oplus Z \rightarrow Y$ por $P(y+z)=y$. temos que $P$ é contínua. Vamos demonstrar esta afirmação usando o Teorema do Gráfico fechado.

Sejam $y_{n}+z_{n} \rightarrow 0$ e $P\left(y_{n}+z_{n}\right) \rightarrow y$. Temos que $P\left(y_{n}+z_{n}\right)=y_{n} \rightarrow y$ e $z_{n}=$ $\left(y_{n}+z_{n}\right)-y_{n}, \operatorname{logo} z_{n} \rightarrow-y$ e como $Z$ é fechado $-y \in Z \operatorname{logo} y \in Y \cap Z$. Portanto $y=0$. Sendo assim segue pelo Teorema do Gráfico Fechado que $P$ é contínua.

Para mostrarmos que a projeção de $Y+Z$ para $Y$ não é contínua construiremos, para todo $\delta>0$, vetores $y \in Y$ e $z \in Z$ tal que $\delta\|y+z\|>\|y-z\|$. Pois neste caso teremos $\left\|\frac{y-z}{\delta\|y+z\|}\right\|<1$ e $P\left(\frac{y-z}{\delta\|y+z\|}\right)=\frac{y}{\delta\|y+z\|}$.

Mas $\left\|\frac{y}{\delta\|y+z\|}\right\| \geq \frac{1-\delta}{2 \delta}$, pois $\|y+z\|=\|-y-z\|=\|-y-z+y-y\| \leq\|-2 y\|+\|y-z\|<$ $\|2 y\|+\delta\|y+z\|$.

Portanto $\|y+z\|<\|2 y\|+\delta\|y+z\|$, logo $(1-\delta)\|y+z\|<2\|y\|$ o que implica que 
$\frac{1-\delta}{2 \delta}<\frac{\|y\|}{\delta\|y+z\|}$

Conclusão: $\|P\|$ não é limitada, logo não é contínua.

Vamos dividir o estudo em dois casos.

caso 1: $Y$ e $Z$ são gerados por bases de blocos.

Consideremos $\delta>0$, e $k \in K$ tal que $f(k)^{-1 / 2}<\delta / 4, k$ existe porque sabemos que $\sqrt{f(k)} \stackrel{k \rightarrow+\infty}{\rightarrow}+\infty$.

Como $X$ satisfaz uma $f$-estimativa inferior temos pelo Lema 3.11 que todo subespaço de bloco de $X$ contém, para todo $\epsilon>0$ e $N \in \mathbb{N}$, um $l_{1+}^{N}$-average com constante $1+\epsilon$. (Tomamos $C=1+\epsilon$ no Lema 3.11 ).

Seja $g$ a função obtida de $\phi$ na Observação 2.10 no caso $K_{0}=K$, então como foi verificado na demonstração do Lema 4.13 temos que todo vetor ou tem a norma do supremo ou satisfaz a inequação $\|E x\| \leq \sup \left\{\left|x^{*}(E x)\right|: M \geq 2, x^{*}\right.$ é uma (M,g)-forma $\}$. Faremos agora as seguintes construções.

Sejam $M_{1}=j_{2 k} \in L, \mu=1 / 40$ e $\epsilon=1 / 10$. Consideremos $x_{1}^{\prime}=x_{11}+\ldots+x_{1 M_{1}}$, onde $x_{11}, \ldots, x_{1 M_{1}}$ é uma RIS com constante $(1+\mu)$ em $Y$. Notemos que para construir a RIS

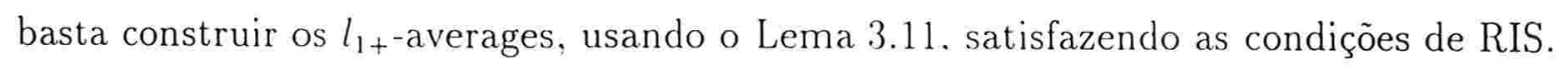

Seja $x_{1}=\frac{x_{1}^{\prime}}{\left\|x_{1}^{\prime}\right\|} \operatorname{logo} x_{1}=\frac{1}{\left\|x_{1}^{\prime}\right\|}\left(x_{11}+\ldots+x_{1 M_{1}}\right)$ é um vetor RIS normalizado com constante $(1+\mu)$ em $Y$. Coloquemos $\alpha_{1}=\frac{1}{\left\|x_{1}^{\prime}\right\|}$.

Análogo ao que foi feito na demonstração do Lema 4.15 temos que $x_{1}$ é um $l_{1+}^{N_{1}}$ average com constante $(1+4 \mu)=(1+\epsilon)$, onde $M_{1}^{\epsilon / 4}=N_{1} \geq 4 M_{f}(k / \epsilon) / \epsilon f^{\prime}(1)$.

Temos que $\frac{x_{1}}{\alpha_{1}}$ é uma RIS com constante $1+\mu, 0<\mu<\frac{1}{2}$, então pelo Lema 3.20 temos que $\left\|E \frac{x_{1}}{\alpha_{1}}\right\| \geq \frac{1}{4}$ para intervalos com $\lambda(E) \geq 1$ e análogo ao que fizemos no capítulo 3 , pág 77 , temos que $\left\|E \frac{x_{1}}{\alpha_{1}}\right\|_{\infty}<\frac{1}{4}$, logo não ocorre a norma do supremo para $\frac{x_{1}}{\alpha_{1}}$. Portanto pela observação feita no começo desta demonstração temos que $\left\|E \frac{x_{1}}{\alpha_{1}}\right\| \leq \sup \left\{\left|x^{*}\left(E \frac{x_{1}}{\alpha_{1}}\right)\right|\right.$ : $M \geq 2, x^{*}$ é uma $(M, g)$-forma $\}$.

Logo pelo Lema 3.21 temos que $\left\|\frac{x_{1}}{\alpha_{1}}\right\| \leq\left(1+\mu+\mu^{\prime}\right) M_{1} g\left(M_{1}\right)^{-1} \leq(1+\epsilon) M_{1} g\left(M_{1}\right)^{-1}$. Portanto $\left\|x_{1}\right\| \leq(1+\epsilon) M_{1} g\left(M_{1}\right)^{-1}\left|\alpha_{1}\right|=(1+\epsilon) M_{1} g\left(M_{1}\right)^{-1} \frac{1}{\left\|x_{1}^{\prime}\right\|}$.

Pelo Lema $4.12 G(x)=x f(x)^{-1}$ se $N \in J \backslash K_{0}$ e $x \in[\ln N, \exp N]$ então $g(x)=$ 
$\frac{x}{\frac{x}{f(x)}}=f(x)$ para todo $x \in L$. Logo não existe diferença entre uma $\left(M_{1}, g\right)$-forma e uma $\left(M_{1}, f\right)$-forma .

Para cada $j$ entre 1 e $M_{1}$ sejam $E_{j}=\operatorname{ran}\left(x_{1 j}\right), x_{1 j}^{*}$ o funcional suporte de $E_{j}\left(\frac{x_{1}}{\alpha_{1}}\right)$ e $x_{1}^{* *}=g\left(M_{1}\right)^{-1} \sum_{j=1}^{M_{1}} x_{1 j}^{*}$, a definição dos funcionais suporte é análoga a feita na demonstração do Corolário 3.17, e como foi visto nesta demonstração $x_{1}^{\prime *}$ é uma $\left(M_{1}, g\right)$-forma.

Então $x_{1}^{\prime *}\left(x_{1}\right)=\alpha_{1} x_{1}^{\prime *}\left(\frac{x_{1}}{\alpha_{1}}\right)=\alpha_{1} g\left(M_{1}\right)^{-1} \sum_{j=1}^{M_{1}} x_{1 j}^{*}\left(\frac{x_{1}}{\alpha_{1}}\right)=\frac{1}{\left\|x_{1}^{\prime}\right\|} g\left(M_{1}\right)^{-1}\left(\left\|E_{1}\left(\frac{x_{1}}{\alpha_{1}}\right)\right\|+\ldots+\right.$ $\left.\left\|E_{M_{1}}\left(\frac{x_{1}}{\alpha_{1}}\right)\right\|\right)=\frac{1}{\left\|x_{1}^{\prime}\right\|} g\left(M_{1}\right)^{-1} M_{1}$. Temos que $\frac{\left\|x_{1}\right\|}{1+\epsilon} \leq \frac{M_{1} g\left(M_{1}\right)^{-1}(1+\epsilon)}{\left\|x_{1}^{\prime}\right\|(1+\epsilon)}=\frac{M_{1} g\left(M_{1}\right)^{-1}}{\left\|x_{1}^{\prime}\right\|}$.

Portanto $x_{1}^{\prime *}\left(x_{1}\right) \geq(1+\epsilon)^{-1}\left\|x_{1}\right\|=\frac{1}{1+\epsilon}=\frac{10}{11}$.

Temos que $x_{1}^{\prime *}\left(x_{1}\right)=\frac{10}{11}$, coloquemos $\beta=\frac{10}{11} \operatorname{logo} \beta \geq \frac{1}{2}$ e $\frac{x_{1}^{\prime}\left(x_{1}\right)}{2 \beta}=\frac{1}{2}$.

Seja $z^{*}=\frac{x_{1}^{\prime}}{2 \beta}, z^{*}$ é uma $\left(M_{1}, g\right)$-forma com $\operatorname{ran}\left(z^{*}\right)=\operatorname{ran}\left(x_{1}^{\prime *}\right)$. De fato, $z^{*}=\sum_{j=1}^{M_{1}} \frac{x_{1 j}^{\prime *}}{2 \beta}, \frac{x_{i j}^{\prime}}{2 \beta}<\ldots<\frac{x_{1 M_{1}}^{\prime}}{2 \beta}$.

$\left\|\frac{x_{1 j}^{\prime}}{2 \beta}\right\|=\frac{1}{2 \beta}\left\|x_{1 j}^{\prime *}\right\| \leq \frac{1}{g\left(M_{1}\right)}$ e $\left\|z^{*}\right\|=\left\|\frac{x_{i}^{\prime}}{2 \beta}\right\|=\frac{1}{2 \beta}\left\|x_{1}^{{ }^{*} *}\right\| \leq 1$. Portanto $z^{*}$ é uma $\left(M_{1}, g\right)$-forma com $\operatorname{ran}\left(z^{*}\right)=\operatorname{ran}\left(x_{1}^{\prime *}\right)$ e $z^{*}\left(x_{1}\right)=\frac{1}{2}$.

Pelo Lema 4.16 temos que existe uma $\left(M_{1}, g\right)$-forma $x_{1}^{*} \in Q$ tal que $\left|x_{1}^{*}\left(x_{1}\right)-1 / 2\right| \leq$ $k^{-1}$ e $\operatorname{ran}\left(x_{1}^{*}\right)=\operatorname{ran}\left(x_{1}\right)$.

Agora seja $M_{2}=\sigma\left(x_{1}^{*}\right)$, e considere um vetor RIS normalizado $x_{2} \in Z$ de comprimento $M_{2}$ com constante $(1+\mu)$ tal que $x_{1}<x_{2}$ ( a construção de $x_{2}$ é análoga a de $x_{1}$ ). Como no caso de $x_{1}$ temos que $x_{2}$ é um $l_{1+}^{N_{2}}$-average com constante $(1+\epsilon)$, onde $N_{2}=M_{2}^{\epsilon / 4}$ e, como acima, podemos achar uma $\left(M_{2}, g\right)$-forma $x_{2}^{*}$ tal que $\left|x_{2}^{*}\left(x_{2}\right)-1 / 2\right| \leq k^{-1} \mathrm{e}$ $\operatorname{ran}\left(x_{2}^{*}\right)=\operatorname{ran}\left(x_{2}\right)$.

Continuando desta maneira, obtemos um par de sequências $x_{1}, \ldots, x_{k}$ e $x_{1}^{*}, \ldots, x_{k}^{*}$ com várias propriedades que precisamos.

1) $x_{i} \in Y$ quando $i$ é ímpar e $x_{i} \in Z$ quando $i$ é par.

2) $\left\|x_{i}\right\|=1$ para qualquer $i$ e $\left\|x_{i}^{*}\right\| \leq 1, x_{i}^{*}$ é uma $\left(M_{i}, g\right)$-forma para todo $i$.

3) $\left|x_{i}^{*}\left(x_{i}\right)-1 / 2\right| \leq 1 / k$ para todo $i$. 
Como na demonstração do Lema 4.15, nossa escolha de $\sigma$ e a baixa limitação para $N_{1}$ garante que $x_{1}, \ldots, x_{k}$ é uma RIS de comprimento $k$. A sequência $x_{1}^{*}, \ldots, x_{k}^{*}$ é uma sequência especial de comprimento $k$, pois $x_{i}^{*} \in Q, \forall i, x_{1}^{*} \in A_{j_{2 k}}^{*}(X)$ já que $x_{1}^{*}$ é uma $\left(M_{1}, f\right)$-forma e $x_{i+1}^{*} \in A_{\sigma\left(x_{i}^{*}, \ldots, x_{i}^{*}\right)}^{*}(X), i=1, \ldots, k-1$.

Segue da definição de norma que $\| \sum_{i=1}^{k} x_{i}|| \geq \sup \left\{\left|\bar{g}\left(E \sum_{i=1}^{k} x_{i}\right)\right|: k \in K, \bar{g} \in B_{k}^{*}(X)\right.$, $E \subset \mathbb{N}\} \geq\left|\bar{g}\left(E \sum_{i=1}^{k} x_{i}\right)\right|$. Tomando $E=\operatorname{ran}(w)$ onde $w=\sum_{i=1}^{k} x_{i}$ temos que $\left|\bar{g}\left(E \sum_{i=1}^{k} x_{i}\right)\right|=$ $\left|f(k)^{-1 / 2} \sum_{j=1}^{k} g_{j}\left(\sum_{i=1}^{k} x_{i}\right)\right| \geq f(k)^{-1 / 2} \sum_{j=1}^{k} g_{j}\left(\sum_{i=1}^{k} x_{i}\right)$

$\left(g_{j}\right)_{j=1}^{k}$ é uma sequência especial de comprimento $k$ então em particular podemos tomar $g_{j}=x_{j}^{*}$, para qualquer $j, 1 \leq j \leq k$. Então continuando temos $f(k)^{-1 / 2} \sum_{j=1}^{k} g_{j}\left(\sum_{i=1}^{k} x_{i}\right)=f(k)^{-1 / 2} \sum_{j=1}^{k} x_{j}^{*}\left(\sum_{i=1}^{k} x_{i}\right)=f(k)^{-1 / 2} \sum_{j=1}^{k} \sum_{i=1}^{k} x_{j}^{*}\left(x_{i}\right)$.

Como $\operatorname{ran}\left(x_{i}^{*}\right)=\operatorname{ran}\left(x_{i}\right)$ segue que $f(k)^{-1 / 2} \sum_{j=1}^{k} \sum_{i=1}^{k} x_{j}^{*}\left(x_{i}\right)=f(k)^{-1 / 2} \sum_{i=1}^{k} x_{i}^{*}\left(x_{i}\right)$. Portanto $\left\|\sum_{i=1}^{k} x_{i}\right\| \geq f(k)^{-1 / 2} \sum_{i=1}^{k} x_{i}^{*}\left(x_{i}\right)$. Mas $\left|x_{i}^{\pi}\left(x_{i}\right)-1 / 2\right| \leq 1 / k \cdot \operatorname{logo} \frac{1}{2}-\frac{1}{k} \leq x_{i}^{*}\left(x_{i}\right) \leq \frac{1}{2}+\frac{1}{k}$ então $x_{i}^{*}\left(x_{i}\right) \geq \frac{1}{2}-\frac{1}{k}$ para todo $i$. Portanto $f(k)^{-1 / 2} \sum_{i=1}^{k} x_{i}^{*}\left(x_{i}\right) \geq f(k)^{-1 / 2}(k / 2-1)$.

A prova estará completa se acharmos para $\left\|\sum_{i=1}^{k}(-1)^{i-1} x_{i}\right\|$ uma limitação superior conveniente. Para isto vamos utilizar o Lema 4.15, mas para usá-lo temos que verificar que $\left|\left(\sum_{i=1}^{k} x_{i}^{*}\right)\left(\sum_{i=1}^{k}(-1)^{i-1} E x_{i}\right)\right| \leq 2$ para todo intervalo $E$.

Podemos verificar que para $x_{i}^{*}\left(E x_{i}\right)$ também temos $\left|x_{i}^{*}\left(E x_{i}\right)-1 / 2\right| \leq 1 / k, \forall i$ então vejamos,

$\left|\left(\sum_{i=1}^{k} x_{i}^{*}\right)\left(\sum_{i=1}^{k}(-1)^{i-1} E x_{i}\right)\right|=\left|\sum_{i=1}^{k}(-1)^{i-1} x_{i}^{*}\left(E x_{i}\right)\right|$, pois como $\operatorname{ran}\left(x_{i}^{*}\right)=\operatorname{ran}\left(x_{i}\right)$ temos que $x_{i}^{*}\left(E x_{j}\right)=0$ para $i \neq j$.

Sabemos que $\left|x_{i}^{*}\left(E x_{i}\right)-\frac{1}{2}\right| \leq \frac{1}{k} \log \frac{1}{2}-\frac{1}{k} \leq x_{i}^{*}\left(E x_{i}\right) \leq \frac{1}{2}+\frac{1}{k} \mathrm{e}-\frac{1}{2}-\frac{1}{k} \leq-x_{i}^{*}\left(E x_{i}\right) \leq$ 
$-\frac{1}{2}+\frac{1}{k}$

Suponha que $k$ é par então,

$\left|\sum_{i=1}^{k}(-1)^{i-1} x_{i}^{*}\left(E x_{i}\right)\right| \leq \frac{k}{2}\left(\frac{1}{2}+\frac{1}{k}-\frac{1}{2}+\frac{1}{k}\right)=\frac{k}{2}\left(\frac{2}{k}\right)=1 \leq 2$.

Suponha que $k$ é ímpar então,

$\left|\sum_{i=1}^{k}(-1)^{i-1} x_{i}^{=}\left(E x_{i}\right)\right| \leq \frac{k-1}{2}\left(\frac{2}{k}\right)+\frac{1}{2}+\frac{1}{k}=\frac{k-1}{k}+\frac{1}{k}+\frac{1}{2}=1+\frac{1}{2} \leq 2$.

Portanto $\left|\left(\sum_{i=1}^{k} x_{i}^{*}\right)\left(\sum_{i=1}^{k}(-1)^{i-1} E x_{i}\right)\right| \leq 2$.

Portanto podemos utilizar o Lema 4.15 já que todas as hipóteses estão satisfeitas.

Com isso construimos $y \in Y$, onde $y$ é a soma dos $x_{i^{\prime} s}$ ímpares, e $z \in Z$, onde $z$ é a soma dos $x_{i^{\prime} s}$ pares, com $\|y+z\|=\left\|\sum_{i=1}^{k} x_{i}\right\| \geq f(k)^{-1 / 2}(k / 2-1) \mathrm{e}$ $\|y-z\|=\left\|\sum_{i=1}^{k}(-1)^{i-1} x_{i}\right\| \leq(1+2 \epsilon) k f(k)^{-1}$.

Temos também que $\frac{\|y-z\| f(k)^{1 / 2}}{3} \leq \frac{(1+2 \epsilon) k f(k)^{-1} f(k)^{1 / 2}}{3}=\frac{(1+2 \epsilon) k f(k)^{-1 / 2}}{3}$. Verificaremos agora que $\frac{(1+2 \epsilon) k}{3} \leq \frac{(k-2)}{2}$.

$\frac{(1+2 \epsilon) k}{3} \leq \frac{(k-2)}{2} \Longleftrightarrow(1+2 \epsilon) 2 k \leq 3(k-2) \Longleftrightarrow 2 k+4 k \epsilon-3 k+6 \leq 0 \Longleftrightarrow-k+4 k \epsilon+6 \leq$ $0 \Longleftrightarrow-k+\frac{4 k}{10}+6 \leq 0 \Longleftrightarrow k \geq 10$, o que é verdade.

Portanto $\frac{(1+2 \epsilon) k f(k)^{-1 / 2}}{3} \leq(k / 2-1) f(k)^{-1 / 2} \leq\|y+z\|$. Sabemos que $f(k)^{-1 / 2}<\delta / 4$ $\operatorname{logo} \sqrt{f(k)}>4 / \delta$. Sendo assim temos que $\frac{f(k)^{1 / 2}}{3}\|y-z\|>\frac{4}{3 \delta}\|y-z\|>\frac{1}{\delta}\|y-z\|=$ $\delta^{-1}\|y-z\|$. Portanto $\|y+z\| \geq \frac{f(k)^{1 / 2}}{3}\|y-z\|>\delta^{-1}\|y-z\|$.

caso 2: Sejam agora $Y$ e $Z$ subespaços quaisquer de $X_{G M}$.

Seja $0<\delta<1$. Lembrando que $\epsilon=1 / 10$ e que $\lim _{k \rightarrow+\infty} \frac{1}{\sqrt{f(k)}}=0 \mathrm{e}$ $\lim _{k \rightarrow+\infty} \sqrt{f(k)}\left(\frac{k / 2-1}{k}\right)=+\infty$, podemos escolher $k \in K$ tal que $f(k)^{-1 / 2}<\delta / 4$ e $\frac{(1+2 \epsilon) k}{f(k)}<$ $\frac{\delta(k / 2-1)}{\sqrt{f(k)}}$.

Seja $c$ o ponto médio do segmento que une $\frac{(1+2 \epsilon) k}{f(k)}$ e $\frac{\delta(k / 2-1)}{\sqrt{f(k)}}$ e seja $\theta, \theta<\frac{1}{2}$ tal que $2 \theta+\frac{(1+2 \epsilon) k}{f(k)}<c$. 
Pelo Teorema 0.10 e sua demonstração temos que existe $\left(y_{n}\right)_{n}$ base de $Y$ equivalente a uma base de blocos $\left(b_{n}\right)_{n}$ de $\left(e_{n}\right)_{n}$, com $\sum_{n=1}^{\infty}\left\|y_{n}-b_{n}\right\| \leq \theta$ e $\left(z_{n}\right)_{n}$ base de $Z$ equivalente a uma base de blocos $\left(B_{n}\right)_{n}$ de $\left(e_{n}\right)_{n}, \operatorname{com} \sum_{n=1}^{\infty}\left\|z_{n}-B_{n}\right\| \leq \theta$.

Consideremos agora $Y^{\prime}=\left[\left(b_{n}\right)_{n}\right]$ e $Z^{\prime}=\left[\left(B_{n}\right)_{n}\right]$. Análogo ao que foi feito no caso 1 temos que existe $\overline{x_{1}}, \overline{x_{2}}, \ldots, \overline{x_{k}}$ tais que os $\overline{x_{i^{\prime} s}}$ ímpares estão em $Y^{\prime}$ e os $\overline{x_{i^{\prime} s}}$ pares estão em $Z^{\prime}$, onde os $\overline{x_{i^{\prime} s}}$ tem a seguinte forma:

$\overline{x_{1}}=\alpha_{1}[\underbrace{\left(b_{11}+b_{12}+\ldots+b_{1 n_{1}}\right)}_{=x_{11}}+\underbrace{\left(b_{21}+b_{22}+\ldots+b_{2 n_{2}}\right)}_{=x_{12}}+\ldots+\underbrace{\left(b_{M_{1} 1}+b_{M_{1} 2}+\ldots+b_{M_{1} n_{M_{1}}}\right)}_{=x_{1 M_{1}}}]$ , onde $b_{i j} \in\left\{b_{n}\right\}$ e $\alpha_{1}=\frac{1}{\left\|x_{11}+\ldots+x_{1 M_{1}}\right\|}$, $\overline{x_{2}}=\alpha_{2}[\underbrace{\left(B_{11}+B_{12}+\ldots+B_{1 m_{1}}\right)}_{=x_{21}}+\underbrace{\left(B_{21}+B_{22}+\ldots+B_{2 m_{2}}\right)}_{=x_{22}}+\ldots+$ $+\underbrace{\left(B_{M_{2} 1}+B_{M_{2} 2}+\ldots+B_{M_{2} m_{M_{2}}}\right)}_{=x_{2 M_{2}}}]$, onde $B_{i j} \in\left\{B_{n}\right\}$ e $\alpha_{2}=\frac{1}{\left\|x_{21}+\ldots+x_{2 M_{2} \|}\right\|}$, e assim por diante.

Com isto construímos $y^{\prime} \in Y^{\prime}$ onde $y^{\prime}$ é a soma dos $\overline{x_{i^{\prime} s}}$ impares e $z^{\prime} \in Z^{\prime}$ onde $z^{\prime}$ é a soma dos $\overline{x_{i^{\prime}} s}$ pares.

Temos pelo que vimos no caso 1 que $\delta\left\|y^{\prime}+z^{\prime}\right\| \geq \frac{\delta(k / 2-1)}{\sqrt{f(k)}},\left\|y^{\prime}-z^{\prime}\right\| \leq \frac{(1+2 \epsilon) k}{f(k)}$ e $\delta\left\|y^{\prime}+z^{\prime}\right\|>\left\|y^{\prime}-z^{\prime}\right\|$.

Sejam $x_{1}=\alpha_{1}\left[\left(y_{11}+y_{12}+\ldots+y_{1 n_{1}}\right)+\left(y_{21}+y_{22}+\ldots+y_{2 n_{2}}\right)+\ldots+\left(y_{M_{1} 1}+y_{M_{1} 2}+\right.\right.$ $\left.\left.\ldots+y_{M_{1} n_{M_{1}}}\right)\right]$ pertencente a $Y$, onde $y_{i j} \in\left\{y_{n}\right\}$, e $i j$ são os indices usados para construir $b_{i j}$

$x_{2}=\alpha_{2}\left[\left(z_{11}+z_{12}+\ldots+z_{1 m_{1}}\right)+\left(z_{21}+z_{22}+\ldots+z_{2 m_{2}}\right)+\ldots+\left(z_{M_{2} 1}+z_{M_{2} 2}+\ldots+z_{M_{2} m_{M_{2}}}\right)\right]$ pertencente a $Z$, onde $z_{i j} \in\left\{z_{n}\right\}$, e $i j$ são os índices usados para construir $B_{i j}$

Procedendo analogamente obtemos $x_{1}, \ldots, x_{k}$. Consideremos $y \in Y$ onde $y$ é a soma dos $x_{i^{\prime} s}$ ímpares e $z \in Z$ onde $z$ é a soma dos $x_{i^{\prime} s}$ pares. Então,

I) $\|y-z\| \leq\left\|y-y^{\prime}\right\|+\left\|y^{\prime}-z^{\prime}\right\|+\left\|z^{\prime}-z\right\| \leq\left\|y^{\prime}-z^{\prime}\right\|+2 \theta<c$.

II) $\left\|y^{\prime}+z^{\prime}\right\| \leq\left\|y^{\prime}-y\right\|+\|y+z\|+\left\|-z+z^{\prime}\right\| \leq\|y+z\|+2 \theta$. Logo $\delta\left\|y^{\prime}+z^{\prime}\right\| \leq$ $\delta\|y+z\|+2 \theta \delta \leq \delta\|y+z\|+2 \theta$, mas $c<\delta\left\|y^{\prime}+z^{\prime}\right\|-2 \theta \leq \delta\|y+z\|$.

Portanto por I) e II) temos que $\|y-z\|<\delta\|y+z\|$. Assim $Y$ e $Z$ não formam uma soma direta topológica, então $X_{G M}$ é hereditariamente indecomponível. 
Corolário 4.18 O espaço de Gowers e Maurey nâo tem nenhuma sequência básica incondicional.

\section{Demonstração}

Suponhamos que existe uma sequência básica incondicional $\left(x_{n}\right)_{n}$ em $X_{G M}$. Seja $W=\left[\left(x_{n}\right)_{n}\right]$ então podemos tomar $Y=\left[\left(x_{2 n}\right)_{n}\right]$ e $Z=\left[\left(x_{2 n-1}\right)_{n}\right] \operatorname{logo} W=Y \oplus Z$, o que é absurdo já que $X_{G M}$ é hereditariamente indecomponível.

Portanto $X_{G M}$ não contém nenhuma sequência básica incondicional.

Proposição $4.19 X_{G M}$ é reflexivo.

\section{Demonstração}

Vamos fazer esta demonstração provando que a base $\left(e_{n}\right)_{n}$ de $X_{G M}$ é shrinking e limitadamente completa.

1) $\left(e_{n}\right)_{n}$ é shrinking.

Suponhamos que $\left(e_{n}\right)_{n}$ não seja shrinking, então existe $x^{*} \in X_{G M}^{*}$ tal que $\left\|\left.x^{*}\right|_{\left(e_{n_{0}}, e_{n_{1}}, \ldots\right]}\right\| \nrightarrow \rightarrow 0$. Podemos supor que $\left\|x^{*}\right\|=1$, pois se $\left\|x^{*}\right\| \neq 1$ basta tomar $\frac{x^{*}}{\left\|x^{*}\right\|}$ e ainda teremos a condição $\left.\| x^{*} \mid \epsilon_{n_{0}}, e_{n_{1}}, \ldots\right] \| \not \rightarrow 0$.

De $\left\|\left.x^{*}\right|_{\left(e_{n_{0}}, e_{n_{1}}, \ldots .\right)}\right\| \nrightarrow 0$ temos que existe $\epsilon>0$, podemos supor que $2 \epsilon<1$, tal que $\forall n_{0}$, existe $n \geq n_{0}$ de modo que $\left\|\left.x^{*}\right|_{\left[e_{n}, e_{n+1}, \ldots\right]}\right\| \geq 2 \epsilon$.

Tomemos $n_{0}=1$ então existe $n_{1}>1 \mathrm{com}\left\|\left.x^{*}\right|_{\left[e_{n_{1}}, e_{n_{1}+1}, \ldots\right]}\right\| \geq 2 \epsilon$, ou seja, existe $v_{1}=\sum_{i=n_{1}}^{\infty} a_{i} e_{i},\left\|v_{1}\right\| \leq 1$, satisfazendo $\left\|x^{*}\left(v_{1}\right)\right\| \geq 2 \epsilon$.

Como $x^{*}\left(v_{1}\right)=\lim _{n \rightarrow+\infty} x^{*}\left(\sum_{i=n_{1}}^{n} a_{i} e_{i}\right)$, segue que existe $n_{2}$ tal que $\left\|x^{*}\left(\sum_{i=n_{1}}^{n_{2}} a_{i} e_{i}\right)\right\| \geq \epsilon$. Seja $w_{1}=\sum_{i=n_{1}}^{n_{2}} a_{i} e_{i}$, então $\left\|x^{*}\left(\frac{w_{1}}{\left\|w_{1}\right\|}\right)\right\| \geq \frac{1}{\left\|w_{1}\right\|} \epsilon \geq \frac{1}{\left\|v_{1}\right\|} \epsilon \geq \epsilon$ já que $w_{1}$ é uma projeção de $v_{1}$ e $\left\|v_{1}\right\| \leq 1$.

Coloquemos $x_{1}=\frac{w_{1}}{\left\|w_{1}\right\|}$. e eventualmente trocando por $-x_{1}$, podemos supor que $x^{*}\left(x_{1}\right) \geq \epsilon$. 
Consideremos $n_{2}$ então existe $n_{3}>n_{2}$ com $\left\|\left.x^{*}\right|_{\left(e_{n_{3}}, e_{n_{3}+1}, \ldots\right]}\right\| \geq 2 \epsilon$, ou seja, existe $v_{2}=\sum_{i=n_{3}}^{\infty} a_{i} e_{i},\left\|v_{2}\right\| \leq 1$, satisfazendo $\left\|x^{*}\left(v_{2}\right)\right\| \geq 2 \epsilon$.

Como $x^{*}\left(v_{2}\right)=\lim _{n \rightarrow+\infty} x^{*}\left(\sum_{i=n_{3}}^{n} a_{i} e_{i}\right)$, segue que existe $n_{4}$ tal que $\left\|x^{*}\left(\sum_{i=n_{3}}^{n_{4}} a_{i} e_{i}\right)\right\| \geq \epsilon$. Seja $w_{2}=\sum_{i=n_{3}}^{n_{4}} a_{i} e_{i}$, então $\left\|x^{*}\left(\frac{w_{2}}{\left\|w_{2}\right\|}\right)\right\| \geq \frac{1}{\left\|w_{2}\right\|} \epsilon \geq \frac{1}{\left\|v_{2}\right\|} \epsilon \geq \epsilon$ já que $w_{2}$ é uma projeção de $v_{2} \mathrm{e}$ $\left\|v_{2}\right\| \leq 1$.

Coloquemos $x_{2}=\frac{w_{2}}{\left\|w_{2}\right\|}$, e eventualmente trocando por $-x_{2}$, podemos supor que $x^{*}\left(x_{2}\right) \geq \epsilon$.

Continuando o raciocínio temos que existe $\left(x_{n}\right)_{n}$ sequência normalizada de blocos, tais que $x^{*}\left(x_{n}\right) \geq \epsilon$.

Afirmação: $\sum_{n \in A} x_{n}$ é um $l_{1+}^{|A|}$ vetor com constante $\frac{1}{\epsilon}, \forall A$ finito contido nos naturais.

Temos que provar que $\left\|x_{n}\right\| \leq \frac{1}{\lfloor|A|}\left\|\sum_{n \in A} x_{n}\right\|$, isto é, $\epsilon|A| \leq\left\|\sum_{n \in A} x_{n}\right\|$. Ora $x^{*}\left(\sum_{n \in A} x_{n}\right)=$ $\sum_{n \in A} x^{*}\left(x_{n}\right) \geq \epsilon|A|$ e como $\left\|\sum_{n \in A} x_{n}\right\|=\sup _{\left\|x^{*}\right\| \leq 1}\left|x^{*}\left(\sum_{n \in A} x_{n}\right)\right|$ temos $\left\|\sum_{n \in A}^{n \in A} x_{n}\right\| \geq \epsilon|A|$. Temos que $\frac{1}{\epsilon}>1$, então seja $\frac{1}{\epsilon}=1+\epsilon_{1}$.

Dado $N \in L$, vamos construir uma RIS, $y_{1}, y_{2}, \ldots, y_{N}$, com constante $1+\epsilon_{1}$ onde cada $y_{i}$ é da forma $\lambda_{i}\left(\sum_{j \in A_{i}} x_{j}\right)$, com $\frac{1}{\lambda_{1}} \leq\left|A_{i}\right|, A_{i}$ finito.

Tomemos $A_{1} \subset \mathbb{N}$ finito com $\left|A_{1}\right|>2\left(1+\epsilon_{1}\right) M_{f}\left(N / \epsilon_{1}^{\prime}\right) / \epsilon_{1}^{\prime} f^{\prime}(1)$ e $y_{1}=\lambda_{1}\left(\sum_{j \in A_{1}} x_{j}\right)$, onde $\lambda_{1}=\frac{1}{\left\|\sum_{j \in A_{1}} x_{j}\right\|}$, portanto $\frac{1}{\lambda_{1}}=\left\|\sum_{j \in A_{1}} x_{j}\right\| \leq\left|A_{1}\right|$. Vamos agora escolher $y_{2}$. Sejam $n_{2} \in \mathbb{N}$ satisfazendo $\frac{\epsilon_{1}^{\prime}}{2} f\left(n_{2}\right)^{1 / 2} \geq \mid$ ran $y_{1} \mid$ e $j_{1}=\max A_{1}$. Tomemos agora $n_{2} x_{j^{\prime} s}$, tais que $j>j_{1}$ e indiquemos por $A_{2}$ o conjunto desses índices. Coloquemos $y_{2}=\lambda_{2}\left(\sum_{j \in A_{2}} x_{j}\right)$, onde $\lambda_{2}=\frac{1}{\left\|\sum_{j \in A_{2}} x_{j}\right\|}$, portanto $\frac{1}{\lambda_{2}}=\left\|\sum_{j \in A_{2}} x_{j}\right\| \leq\left|A_{2}\right|$.

Continuando o raciocínio construímos a RIS $y_{1}, \ldots, y_{N}$. 
$\operatorname{Logo} x^{*}\left(y_{1}+\ldots+y_{N}\right)=x^{*}\left(\lambda_{1}\left(\sum_{j \in A_{1}} x_{j}\right)+\lambda_{2}\left(\sum_{j \in A_{2}} x_{j}\right)+\ldots+\lambda_{N}\left(\sum_{j \in A_{N}} x_{j}\right)\right)=\lambda_{1}\left(\sum_{j \in A_{1}} x^{*}\left(x_{j}\right)\right)+$ $\lambda_{2}\left(\sum_{j \in A_{2}} x^{*}\left(x_{j}\right)\right)+\ldots+\lambda_{N}\left(\sum_{j \in A_{N}} x^{*}\left(x_{j}\right)\right) \geq \lambda_{1} \epsilon\left|A_{1}\right|+\lambda_{2} \epsilon\left|A_{2}\right|+\ldots+\lambda_{N} \epsilon\left|A_{N}\right|$ e como $\frac{1}{\lambda_{i}} \leq\left|A_{i}\right|$ temos que $\lambda_{i}\left|A_{i}\right| \geq 1$, portanto $\lambda_{1} \epsilon\left|A_{1}\right|+\lambda_{2} \epsilon\left|A_{2}\right|+\ldots+\lambda_{N} \epsilon\left|A_{N}\right| \geq \epsilon+\epsilon+\ldots+\epsilon=\epsilon N$.

Então em particular temos que $\left\|y_{1}+\ldots+y_{N}\right\| \geq N \epsilon$. E portanto para $N$ suficientemente grande temos uma contradição pelo Lema 4.13. Pois por este Lema temos, $N \epsilon \leq\left\|\sum_{i=1}^{N} y_{i}\right\| \leq\left(1+\epsilon_{1}+\epsilon_{1}^{\prime}\right) N f(N)^{-1}$, isto é, $f(N) \leq \frac{\left(1+\epsilon_{1}+\epsilon_{1}^{\prime}\right)}{\epsilon}$, absurdo para $N$ suficientemente grande.

Portanto $\left(e_{n}\right)_{n}$ é shrinking.

2) $\left(e_{n}\right)_{n}$ é limitadamente completa.

Seja $\left(a_{n}\right)_{n}$ sequência em $\mathbb{R}$, tal que $\sum_{n=1}^{N} a_{n} e_{n}$ é limitada, ou seja, existe $k$ tal que $\left\|\sum_{n=1}^{N} a_{n} e_{n}\right\| \leq k$ para qualquer $N$.

Mostraremos que $\sum_{n=1}^{\infty} a_{n} \epsilon_{n}$ converge, isto é. $\left(S_{n}\right)_{n}$ é de Cauchy em $X_{G M}$ onde $S_{n}=$ $\sum_{i=1}^{n} a_{i} e_{i}$. Suponhamos que $\left(S_{n}\right)_{n}$ não é de Cauchy. Portanto existe $\epsilon>0$ tal que para qualquer $m \in \mathbb{N}$, existe $m_{1}, m_{2} \geq m$ tal que $\left\|S_{m_{2}}-S_{m_{1}}\right\| \geq \epsilon$. Seja $M \in \mathbb{N}$ tal que $\frac{M}{f(M)}>\frac{k}{\epsilon}$ e consideremos os seguintes intervalos $E_{i}=\{M+i\}$ com $1 \leq i \leq M$.

Pela definição de norma temos

$k \geq\left\|S_{M+M}\right\| \geq \frac{1}{f(M)} \sum_{i=1}^{M}\left\|E_{i}\left(S_{M+M}\right)\right\|=\frac{1}{f(M)} \sum_{i=1}^{M}\left\|S_{i+M}-S_{(i-1)+M}\right\| \geq \frac{1}{f(M)} \epsilon M ;$ absurdo.

Portanto $\left(S_{n}\right)_{n}$ é de Cauchy, logo $\left(e_{n}\right)_{n}$ é limitadamente completa.

Como já mencionamos na introdução desta dissertação ainda não é conhecida a solução do seguinte problema.

Problema 4.20 Existe espaço de Banach HI não separável? 


\section{Capítulo 5}

\section{Operadores sobre espaços hereditariamente indecomponíveis}

Neste capítulo estudaremos algumas propriedades de operadores sobre espaços hereditariamente indecomponíveis complexos e reais. A partir dessas propriedades demonstraremos que espaços hereditariamente indecomponíveis tanto reais como complexos são contra-exemplos para o Problema do Hiperplano, isto é, eles não são isomorfos a nenhum de seus hiperplanos.

Inicialmente estudaremos os operadores sobre espaços hereditariamente indecomponíveis complexos e para isto precisamos de algumas definições e Lemas relacionados com a Teoria Espectral.

A teoria apresentada neste capítulo pode ser encontrada em [11], exceto a definição 5.10 que se encontra em [14].

Definição 5.1 Dizemos que $T \in L(X, Y) \operatorname{com} T(X)$ fechado é um operador de Fredholm se o indice de $T$ é finito, isto é colocando $\alpha(T)=\operatorname{dim} \operatorname{Ker} T$ e $\beta(T)=\operatorname{dim} \frac{Y}{T(X)}$ então $i(T)=\alpha(T)-\beta(T)$ é finito.

Definição 5.2 Seja $T \in L(X), \lambda \in \mathbb{C}$ é infinitamente singular para $T$ se $\forall \epsilon>0, \exists Y_{\epsilon}$ subespaço fechado de dimensão infinita de $X$ tal que $\left\|\left.(T-\lambda I)\right|_{Y_{\epsilon}}\right\| \leq \epsilon$.

Lema 5.3 Seja $T \in L(X)$ são equivalentes: 
1) T não é um isomorfismo quando restrito a qualquer subespaço de codimensão finita de $X$.

2) $\forall \epsilon>0, \exists Y_{\epsilon}$ subespaço fechado de dimensão infinita de $X$ tal que $\left\|\left.T\right|_{Y_{\epsilon}}\right\| \leq \epsilon$.

\section{Demonstração:}

1) $\rightarrow$ 2) Segue do Teorema 0.22 .

2) $\rightarrow$ 1) Vamos negar 1) e chegar na negação de 2).

Seja $Y$ um subespaço de codimensão finita de $X$. Suponhamos que $\left.T\right|_{Y}$ é um isomorfismo, então existe $\epsilon>0$ tal que $\epsilon\|y\| \leq\|T(y)\|, \forall y \in Y$. Seja $Z$ um subespaço fechado de dimensão infinita de $X$, pelo Teorema 0.20 existe $V$ subespaço de $X$ de dimensão finita tal que $Z=Z \cap Y \oplus V$.

Portanto existe $z \in Z \cap Y,\|z\|=1$. logo $\|T(z)\| \geq \epsilon$. Sendo assim, $\left\|\left.T\right|_{Z}\right\| \geq \epsilon$.

Lema 5.4 Sejam $T \in L(X)$ e $F_{T}=\{\lambda \in \mathbb{C}: \lambda$ não é infinitamente singular para $T\}$. Então:

1) $F_{T}$ é aberto em $\mathbb{C}$.

2) $\operatorname{Ker}(T-\lambda I)$ é de dimensão finita quando $\lambda \in F_{T}$.

\section{Demonstração}

1) De fato, seja $\lambda_{0} \in F_{T} \operatorname{logo}$ existe $Z$ subespaço de $X$ de codimensão finita tal que $T-\lambda_{0} I$ restrito a $Z$ é um isomorfismo. isto é, $\exists k \in \mathbb{R}$ tal que $k\|z\| \leq\left\|T z-\lambda_{0} z\right\|, \forall z \in Z$.

Seja $\lambda$ tal que $\left|\lambda-\lambda_{0}\right|<\frac{k}{2}$, como $T z-\lambda_{0} z=T z-\lambda z+\lambda z-\lambda_{0} z$ temos que $k\|z\| \leq$ $\left\|T z-\lambda_{0} z\right\| \leq\|T z-\lambda z\|+\left|\lambda-\lambda_{0}\right|\|z\|$, logo $k\|z\| \leq\|T z-\lambda z\|+\frac{k}{2}\|z\|$. Portanto $\frac{k}{2}\|z\| \leq\|T z-\lambda z\|, \forall z \in Z$, isto é, $\lambda \in F_{T}$.

2) De fato, existe $Z$ subespaço de $X$ de codimensão finita tal que $T-\left.\lambda I\right|_{Z}$ é um isomorfismo.

Como o $\operatorname{Ker}(T-\lambda I) \cap Z=\{0\}$, pois $T-\left.\lambda I\right|_{Z}$ é um isomorfismo, segue que $\operatorname{Ker}(T-\lambda I)$ só pode ter uma quantidade finita de vetores linearmente independente, logo $\operatorname{dim} \operatorname{Ker}(T-\lambda I)$ é finita.

Lema 5.5 Seja $T \in L(X)$ se $\lambda \in F_{T}$ e se $\left(x_{n}\right)_{n}$ é uma sequência limitada tal que (T- 
$\lambda I) x_{n}$ é convergente na norma, então $\left(x_{n}\right)_{n}$ tem uma subsequência convergente na norma; além disso, a imagem de qualquer subespaço fechado de $X$ por $T-\lambda I$ é fechado.

\section{Demonstração:}

Seja $S=T-\lambda I$, como $\lambda \in F_{T}$ existe $Y$ subespaço de $X$ de codimensão finita tal que $\left.S\right|_{Y}$ é um isomorfismo sobre a imagem.

Sejam $X=Y \oplus Z$, onde $Z$ é um subespaço de dimensão finita de $X$, e $x_{n}=y_{n}+z_{n}$ com $y_{n} \in Y$ e $z_{n} \in Z$, então $S\left(x_{n}\right)=S\left(y_{n}+z_{n}\right)=S\left(y_{n}\right)+S\left(z_{n}\right)$.

Consideremos $P: X \rightarrow Z$ uma projeção, $\operatorname{logo} z_{n}=P\left(x_{n}\right)$ e portanto $\left\|z_{n}\right\|=$ $\left\|P\left(x_{n}\right)\right\| \leq\|P\|\left\|x_{n}\right\|$.

Como $\left(x_{n}\right)_{n}$ é limitada temos que $\left(z_{n}\right)_{n}$ também é limitada. consequentemente existe subsequência $\left(z_{n_{k}}\right)_{k}$ que converge, logo $S\left(z_{n_{k}}\right)$ é convergente, pois $S$ é contínuo.

Desde que $S\left(z_{n_{k}}\right)$ é convergente, segue que $S\left(y_{n_{k}}\right)=S\left(x_{n_{k}}\right)-S\left(z_{n_{k}}\right)$ é convergente, mas $\left.S\right|_{Y}$ é isomorfismo logo $\left(y_{n_{k}}\right)_{k}$ é convergente, consequentemente $x_{n_{k}}=y_{n_{k}}+z_{n_{k}}$ também é convergente.

Para terminar a demonstração consideremos $F$ um subespaço fechado de $X$, pelo Teorema 0.20 existe $G$ subespaço de $X$ de dimensão finita tal que $F=F \cap Y \oplus G$.

Portanto $S(F)=S(F \cap Y \oplus G)=S(F \cap Y)+S(G)$.

Temos que $S(G)$ é de dimensão finita porque $G$ é de dimensão finita e $S$ é linear e $S(F \cap Y)$ é fechado porque $F \cap Y$ é fechado em $Y$ e $\left.S\right|_{Y}$ é um isomorfismo, logo $\left.S\right|_{F \cap Y}$ ainda é um isomorfismo.

Vamos definir $\bar{T}: X \rightarrow \frac{X}{S(F \cap Y)}$ pondo $\bar{T}(x)=\bar{x}$, claramente $\bar{T}$ é linear e como $\|\bar{T}(x)\|=\|\bar{x}\|=\inf _{u \in \bar{x}}\|u\| \leq\|x\|$ temos que $\bar{T}$ é contínua.

Então como $S(G)$ é de dimensão finita e $\bar{T}$ é linear temos que $\bar{T}(S(G))$ é de dimensão finita e com isto $\bar{T}(S(G))$ é fechado, consequentemente $\bar{T}^{-1} \bar{T}(S(G))=S(G)+S(F \cap Y)$ é fechado.

Lembrando que $S_{p}(T)$ é o espectro de $T$, veja definição $0.2 \bar{\imath}$. e indicando por $\partial S_{p}(T)$ a sua fronteira temos 
Lema 5.6 Seja $T \in L(X)$ se $\lambda \in \partial S_{p}(T) \cap F_{T}$, então $\lambda$ é um autovalor de $T$ com multiplicidade finita.

\section{Demonstração:}

Como $\lambda \in \partial S_{p}(T)$ temos pelo Teorema 0.29 que $\lambda$ é um autovalor aproximado de $T$, isto é, existe uma sequência $\left(x_{n}\right)_{n}$ de norma 1 tal que $(T-\lambda I)\left(x_{n}\right) \stackrel{n \rightarrow+\infty}{\rightarrow} 0$.

A sequência $\left(x_{n}\right)_{n}$ é limitada. Então pelo Lema $5.5,\left(x_{n}\right)_{n}$ tem uma subsequência convergente, ou seja, $\exists x_{i}$, convergente para $x$, logo $(T-\lambda I) x_{i}, \rightarrow(T-\lambda I) x$.

Mas como $\left(x_{i},\right)_{j}$ é subsequência de $\left(x_{n}\right)_{n}$ e $(T-\lambda I) x_{n} \rightarrow 0$ temos $(T-\lambda I) x_{i}, \rightarrow 0$ logo $(T-\lambda I) x=0$. ou seja, $T x=\lambda x$.

Com isso temos que $x$ é um autovetor com autovalor $\lambda$. A multiplicidade de $\lambda$ é a dimensão do $\operatorname{Ki} \operatorname{er}(T-\lambda I)$ que é finita pois $\lambda \in F_{T}$.

Lema 5.7 Seja $T \in L(X)$ se $\lambda \in \partial S_{p}(T) \cap F_{T}$ então $\lambda$ é um ponto isolado do $S_{p}(T)$.

\section{Demonstração:}

Como $F_{T}$ é aberto basta mostrarmos que $\lambda$ é um ponto isolado de $\partial S_{p}(T) \cap F_{T}$, pois se $\lambda$ é ponto isolado da $\partial S_{p}(T) \cap F_{T}$ é porque existe um aberto $A$ tal que $A \cap \partial S_{p}(T) \cap$ $F_{T}=\{\lambda\}, \operatorname{logo} A \cap F_{T} \cap \partial S_{p}(T)=\{\lambda\}$ onde $A \cap F_{T}$ é aberto.

Suponhamos que $\lambda$ não é ponto isolado de $\partial S_{p}(T) \cap F_{T}$.

Como $\lambda$ não é ponto isolado, existe uma sequência $\left(\lambda_{n}\right)_{n} \in \partial S_{p}(T) \cap F_{T}$ que converge para $\lambda, \operatorname{com} \lambda_{n} \neq \lambda$ para todo $n$.

Desde que $\lambda_{n} \in F_{T} \cap \partial S_{p}(T)$, temos pelo Lema 5.6 que $\lambda_{n}$ é um autovalor de $T$.

Seja $x_{n},\left\|x_{n}\right\|=1$, um autovetor para $\lambda_{n}$. Como $T x_{n}-\lambda x_{n}=\lambda_{n} x_{n}-\lambda x_{n}=\left(\lambda_{n}-\lambda\right) x_{n}$ temos que $(T-\lambda I)\left(x_{n}\right) \stackrel{n \rightarrow+\infty}{\rightarrow} 0$, logo como $\left(x_{n}\right)_{n}$ é limitada podemos assumir pelo Lema 5.5 que $x_{n}$ é convergente na norma para algum $x,\|x\|=1$. Então temos que $x_{n} \rightarrow x \operatorname{logo}$ $(T-\lambda I) x_{n} \rightarrow(T-\lambda I) x$, mas $(T-\lambda I) x_{n} \rightarrow 0$ portanto $(T-\lambda I) x=0$, ou seja, $T x=\lambda x$.

Sejam $Y=\left[\left(x_{n}\right)_{n}\right]$ e $U=\left.(T-\lambda I)\right|_{Y}$. Temos que $\left(x_{n}\right) \in Y$ e $x_{n} \rightarrow x$ logo $x \in Y$. pois $Y$ é fechado. 
$U(Y) \subset Y$, pois $U\left(x_{n}\right)=(T-\lambda I)\left(x_{n}\right)=T x_{n}-\lambda x_{n}=\lambda_{n} x_{n}-\lambda x_{n}=\left(\lambda_{n}-\lambda\right) x_{n}$, logo $U\left(x_{n}\right)=x_{n}\left(\lambda_{n}-\lambda\right)$ e pela continuidade de $U$ segue que $U(Y) \subset Y$.

Como $(T-\lambda I)(Y)=U(Y)$ e $\lambda \in F_{T}$ segue do Lema 5.5 que $U(Y)$ é fechado, mas $U\left(x_{n}\right)=\alpha x_{n} \operatorname{logo} x_{n} \subset U(Y), \forall n$. Então pela definição de $Y$ temos $Y=\left[\left(x_{n}\right)_{n}\right] \subset$ $U(Y)$, consequentemente $U Y=Y$. Como já vimos anteriormente $(T-\lambda I) x_{n} \rightarrow 0 \mathrm{e}$ $(T-\lambda I) x_{n} \rightarrow(T-\lambda I) x$ então $U(x)=0, \operatorname{logo} x \in \operatorname{Ker} U=\left.\operatorname{Ker}(T-\lambda I)\right|_{Y}$ e como $\lambda \in F_{T}$ temos que $\operatorname{Ker} U$ tem dimensão finita. Seja $Y_{0}=K \operatorname{er} U$.

Escrevendo $Y=Y_{0} \oplus Y_{1}$, temos $U(Y)=U\left(Y_{0} \oplus Y_{1}\right)=U\left(Y_{0}\right)+U\left(Y_{1}\right)$ mas como $U\left(Y_{0}\right)=0$ temos que $U(Y)=U\left(Y_{1}\right)$, e como $U(Y)=Y$ segue que $U\left(Y_{1}\right)=Y$. Para $\epsilon$ suficientemente pequeno ainda temos $(U-\epsilon I)\left(Y_{1}\right)=Y$, pois $U: Y \rightarrow Y$ é linear, contínua e sobrejetora então considerando $\left.U\right|_{Y_{1}}: Y_{1} \rightarrow Y$ temos que $\left.U\right|_{Y_{1}}$ é linear, contínua, sobrejetora e injetora.

Então pelo Teorema da Aplicação Aberta temos que $\left.U\right|_{Y_{1}}$ é um isomorfismo. Desde que $\left\|\left.U\right|_{Y_{1}}-\left(\left.U\right|_{Y_{1}}-\epsilon I\right)\right\|=\epsilon$ temos que pelo Teorema 0.30 que para $\epsilon<\frac{1}{\left\|U^{-1} \mid Y_{1}\right\|}$ $(U-\epsilon I)\left(Y_{1}\right)$ também é um isomorfismo, ou seja, é bijetor logo $(U-\epsilon I)\left(Y_{1}\right)=Y$.

Mas como para $\epsilon \neq 0$ e $\forall y_{0} \in Y_{0},(U-\epsilon I)\left(\frac{y_{0}}{-\epsilon}\right)=y_{0}$ temos $(U-\epsilon I)\left(Y_{0}\right)=Y_{0}$.

Visto que $(L-\epsilon I)\left(Y_{1}\right)=Y$ temos $(U-\epsilon I)(Y)=(U-\epsilon I)\left(Y_{0} \oplus Y_{1}\right)=Y_{0}+Y=Y$.

Seja $y_{0} \neq 0, y_{0} \in Y_{0}$ então $(U-\epsilon I)\left(\frac{y_{0}}{-\epsilon}\right)=y_{0}$. mas sabemos que $(U-\epsilon I)\left(Y_{1}\right)=Y$ o que implica que $(U-\epsilon I)\left(y_{1}\right)=y_{0}$ para algum $y_{1} \neq 0, y_{1} \in Y_{1}$ e portanto $(U-\epsilon I)\left(\frac{y_{0}}{-\epsilon}-y_{1}\right)=0$, onde $\frac{y_{0}}{-\epsilon}-y_{1} \neq 0$.

Assim temos $\operatorname{Ker}(U-\epsilon I) \neq\{0\}$ para todo $\epsilon$ pequeno, contradizendo o fato de que $\lambda \in \partial S_{p}(T)$, pois como $\operatorname{Ker}(U-\epsilon I) \neq\{0\}$ existe $z \neq 0, z \in X$ tal que $(U-\epsilon I)(z)=0$ logo $(T-\lambda I-\epsilon I)(z)=0$, ou seja, $T(z)=(\lambda+\epsilon) z$.

Como $\operatorname{Ker}(T-(\lambda+\epsilon) I) \neq\{0\}$ temos que $T-(\lambda+\epsilon) I$ não é injetora logo $T-(\lambda+\epsilon) I$ não é bijetora, assim $T-(\lambda+\epsilon) I$ não é inversível então $\lambda+\epsilon \in S_{p}(T)$, absurdo pois $\lambda \in \partial S_{p}(T)$.

Lema 5.8 Seja $T \in L(X)$. Suponha que $S_{p}(T)=\{0\}$. Se $X$ é de dimensão infinita então zero é infinitamente singular para $T$.

\section{Demonstração:}


Suponhamos que $0 \in F_{T}$ mas que $X$ é de dimensão infinita. Então $\left.T\right|_{Z}$ é um isomorfismo sobre a imagem para algum subespaço $Z$ de $X$ de codimensão finita.

Como é isomorfismo temos que $k\|z\| \leq\|T(z)\| \leq l\|z\|, \forall z \in Z$ e algum $k, l \in \mathbb{R}$. Portanto $\|z\| \leq\left\|\frac{T}{k}(z)\right\|$. Logo trocando $T$ por um multiplo de $T$ podemos supor que $\|z\| \leq\|T(z)\|, \forall z \in Z$.

Agora vamos definir $Z_{0}=Z, Z_{1}=Z \cap T(Z), \ldots, Z_{k+1}=Z \cap T\left(Z_{k}\right)$. Todos estes subespaços de $X$ são de dimensão infinita, pois $X=Z \oplus V$ onde $\operatorname{dim} V<\infty$. Como $\operatorname{dim} X=\infty$ e $\operatorname{dim} V<\infty$ temos $\operatorname{dim} Z=\infty$.

$T(Z)=Z \cap T(Z) \oplus W$, onde $\operatorname{dim} W<\infty$ e $Z \cap T(Z)=Z_{1}$. T é isomorfismo e $Z$ é de dimensão infinita $\operatorname{logo} \operatorname{dim} T(Z)=\infty$, e como a dimensão de $W$ é finita temos que a dimensão de $Z_{1}$ é infinita, prosseguindo neste raciocínio temos o resultado.

Seja $z \neq 0$ um elemento de $Z_{k}$, então $z=T^{k}\left(z_{0}\right)$ para algum $z_{0} \neq 0, z_{0} \in Z$. De fato, $z=T\left(z_{k-1}\right)=T\left(T\left(z_{k-2}\right)\right)=\ldots \ldots \ldots=T^{k}\left(z_{0}\right)$ e $0<\left\|z_{0}\right\| \leq\left\|T\left(z_{0}\right)\right\| \leq\left\|T^{2}\left(z_{0}\right)\right\| \leq \ldots \leq$ $\left\|T^{k}\left(z_{0}\right)\right\|$.

Portanto $\left\|z_{0}\right\| \leq\left\|T^{k}\left(z_{0}\right)\right\|$, dividindo por $\left\|z_{0}\right\|$ temos $1 \leq\left\|T^{k} \frac{\left(z_{0}\right)}{\left\|z_{0}\right\|}\right\|$, isto é, $1 \leq\left\|T^{k}\right\|$.

Contradizendo o fato de que $0=r_{S_{p}(T)}=\lim _{n \rightarrow+\infty} \sup \left(\left\|T^{n}\right\|\right)^{1 / n}$, onde $r_{S_{p}(T)}$ é o raio espectral de $T$. O raio espectral de $T$ é igual a zero porque $r_{S_{p}(T)}=\sup |\lambda|, \lambda \in S_{p}(T)$ e como $S_{p}(T)=\{0\}$ temos que $r_{S_{p}(T)}=0$.

Lema 5.9 Se $X$ é de dimensão infinita então $F_{T} \neq \mathbb{C}$.

\section{Demonstração:}

Suponhamos que $F_{T}=\mathbb{C}$. Segue do Lema 5.7 que cada ponto na fronteira do $S_{p}(T)$ é isolado no $S_{p}(T)$, logo $S_{p}(T)$ é finito pelo Teorema de Bolzano-Weierstrass.

Vamos então escrever $S_{p}(T)=\left\{\lambda_{1}, \lambda_{2}, \ldots, \lambda_{n}\right\}$ e consideremos o polinômio $P=(z-$ $\left.\lambda_{1}\right)\left(z-\lambda_{2}\right) \ldots .\left(z-\lambda_{n}\right)$.

Para todo $\lambda \neq 0$ podemos escrever $P-\lambda=\left(z-\mu_{1}\right)\left(z-\mu_{2}\right) \ldots .\left(z-\mu_{n}\right)$ onde $\mu_{i} \notin S_{p}(T)$, pois apenas $\lambda_{1}, \lambda_{2}, \ldots ., \lambda_{n} \in S_{p}(T)$, os $\mu_{i^{\prime} \text { s }}$ são raízes de $P-\lambda$.

Logo $(P-\lambda)(T)=\left(T-\mu_{1} I\right)\left(T-\mu_{2} I\right) \ldots .\left(T-\mu_{n} I\right)=P(T)-\lambda I$ tem inversa contínua, para qualquer $\lambda \neq 0$. A imagem de $(P-\lambda)(T)$ é densa em $X$, pois a imagem de 
$\left(T-\mu_{n} I\right) X$ é denso em $X$ e $\left(T-\mu_{n} I\right)$ é contínua e sobrejetora e sabemos que a imagem de um denso por uma função contínua e sobrejetora é denso. Portanto $S_{p}(P(T))=\{0\}$.

Como $X$ é de dimensão infinita temos pelo Lema 5.8 que zero é infinitamente singular para $P(T)$, isto é, $0 \notin F_{P(T)}$.

Portanto existe uma sequência básica normalizada $\left(x_{n}\right)_{n}$ tal que $P(T) x_{n} \stackrel{n \rightarrow+\infty}{\rightarrow} 0$ ( ver demonstração do Teorema 0.22).

Escrevendo $P(T)=\left(T-\lambda_{1} I\right)\left(T-\lambda_{2} I\right) \ldots . .\left(T-\lambda_{n-1} I\right)\left(T-\lambda_{n} I\right)$ e fazendo $n$ repetidas aplicações do Lema 5.5 , temos que existe $\left(x_{n_{k}}\right)_{k}$ subsequência convergente de $\left(x_{n}\right)_{n}$. O que é absurdo, pois $\left(x_{n}\right)_{n}$ é sequência básica então $\left\|\sum_{i=1}^{n} a_{i} x_{i}\right\| \leq k\left\|\sum_{i=1}^{m} a_{i} x_{i}\right\| \operatorname{logo}\left\|x_{n}\right\| \leq$ $k\left\|x_{n}-x_{m}\right\|, n<m$.

Mas $1=\left\|x_{n}\right\| \leq k\left\|x_{n}-x_{m}\right\|$ então $\left\|x_{n}-x_{m}\right\| \geq \frac{1}{k}$. Portanto $\left(x_{n}\right)_{n}$ não é Cauchy $\operatorname{logo}\left(x_{n}\right)_{n}$ não é convergente.

Na Proposição 5.12 será apresentada uma visão geométrica dos espaços hereditariamente indecomponiveis, mas antes de enunciá-la definiremos ângulo entre subespaços e provaremos uma Proposição.

Definição 5.10 Sejam $Y$ e $Z$ subespaços fechados de dimensão infinita. $\gamma(Y, Z)=\inf \{\|y-z\|:\|y\|=\|z\|=1, y \in Y, z \in Z\}$ é definido como sendo o ângulo entre $Y$ e $Z$.

Proposição 5.11 Sejam $Y$ e $Z$ subespaços fechados de dimensão infinita de um espaço de Banach $X$ tal que $Y \cap Z=\{0\}$. Então $Y+Z$ é fechado se, e somente se, $\gamma(Y, Z)>0$.

\section{Demonstração:}

$\Rightarrow$ ) Suponha que $Y+Z$ é fechado e defina $T: Y \oplus Z \rightarrow Y+Z$ por $T(y, z)=y-z$, $Y \oplus Z$ com a norma infinito.

1) Claramente $T$ é linear.

Como $Y+Z$ é fechado, basta mostrarmos que o gráfico de $T$ é fechado para termos pelo Teorema do Gráfico Fechado que $T$ é contínua. 
2) T é contínua.

De fato, sejam $\left(y_{n}, z_{n}\right) \rightarrow(0,0)$ e $T\left(y_{n}, z_{n}\right) \rightarrow x$. Como $\left(y_{n}, z_{n}\right) \rightarrow(0,0)$ temos que $y_{n} \rightarrow 0$ e $z_{n} \rightarrow 0$ logo $T\left(y_{n}, z_{n}\right)=y_{n}-z_{n} \rightarrow 0$ e assim segue que $x=0$. Portanto pelo Teorema do Gráfico Fechado temos que $T$ é contínua.

3) $T$ é injetora.

De fato, $T(y, z)=0 \Longleftrightarrow y-z=0 \Longleftrightarrow y=z$ e como $Y \cap Z=\{0\}$ temos $y=z=0$.

4) $T$ é sobre a imagem.

Então pelo Teorema da Aplicação Aberta $T$ é um isomorfismo, logo existe $k>0$ tal que $k\|(y, z)\|_{\infty} \leq\|T(y, z)\|$, ou seja, $k \max (\|y\|,\|z\|) \leq\|T(y, z)\|$. Em particular, se $\|y\|=\|z\|=1$, temos $\|y-z\| \geq k>0$. Portanto, inf $\{\|y-z\|:\|y\|=\|z\|=1, y \in$ $Y, z \in Z\}>0$.

$\Leftarrow)$ Defina $T: Y \oplus Z \rightarrow Y+Z$ por $T(y, z)=y-z, Y \oplus Z$ com a norma infinito.

1) Claramente $T$ é linear.

2) $T$ é contínua.

De fato, $\|T(y, z)\|=\|y-z\| \leq\|y\|+\|z\| \leq 2\|(y, z)\|_{\infty}$.

3) $T$ é injetora.

De fato, $T(y, z)=0 \Longleftrightarrow y-z=0 \Longleftrightarrow y=z$ e como $Y \cap Z=\{0\}$ temos $y=z=0$.

4) $T$ é sobre a imagem.

Suponhamos que $T$ não tem inversa contínua. Então existe $\left(m_{i}, n_{i}\right) \in Y \oplus Z$, $\max \left(\left\|m_{i}\right\|,\left\|n_{i}\right\|\right)=1$ tal que $\left\|m_{i}-n_{i}\right\| \stackrel{i \rightarrow+\infty}{\rightarrow} 0$.

Escolha $i_{j}$ tal que $\left\|m_{i},\right\|=1$. Como $1=\left\|m_{i},\right\|=\left\|m_{i,}-n_{i,}+n_{i},\right\| \leq\left\|m_{i,}-n_{i,}\right\|+$ $\left\|n_{i},\right\|$, temos $1-\left\|m_{i},-n_{i},\right\| \leq\left\|n_{i},\right\| \leq 1$, logo $\left\|n_{i},\right\| \stackrel{j \rightarrow+\infty}{\rightarrow} 1$. Sendo assim podemos supor que $n_{i,} \neq 0, \forall j$.

$\left\|m_{i_{j}}-\frac{n_{i j}}{\left\|n_{i},\right\|}\right\|=\left\|m_{i,}-n_{i,}+n_{i,}-\frac{n_{i j}}{\left\|n_{i j}\right\|}\right\| \leq\left\|m_{i,}-n_{i,}\right\|+\left\|n_{i,}-\frac{n_{i,}}{\left\|n_{i},\right\|}\right\|$.

Fazendo $j \rightarrow+\infty$ temos que $\left\|m_{i_{j}}-\frac{n_{i}}{\left\|n_{i},\right\|}\right\|=0$, absurdo pois deveriamos ter $\| m_{i}$, $\frac{n_{i}}{\left\|n_{i},\right\|} \|>0$ 
Portanto $T$ tem inversa contínua. Logo $T$ é um isomorfismo e com isto temos que $Y+Z$ é fechado.

Proposição 5.12 X' é hereditariamente indecomponivel se, e somente se, para qualquer $Y$ e $Z$ subespaços fechados de dimensão infinita de $X$, e para qualquer $\epsilon>0$ existe $y \in Y$, $z \in Z,\|y\|=\|z\|=1$ e $\|y-z\|<\epsilon$.

\section{Demonstração:}

$\Rightarrow$ S Suponhamos que não, então existem $Y, Z$ subespaços fechados de dimensão infinita de $X$ e $\epsilon>0$ tal que para qualquer $y \in Y, z \in Z$ com $\|y\|=\|z\|=1$, temos $\|y-z\| \geq \epsilon>0$.

1) $Y \cap Z=\{0\}$. pois se $x \in Y \cap Z$ então $\|x-x\| \geq \epsilon$, absurdo.

2) $Y+Z$ é fechado em $X$ pela Proposição 5.11 .

Então tomando $W=Y+Z$ temos que $W$ é fechado e $W=Y \oplus Z$, absurdo pois $X$ é hereditariamente indecomponível.

$\Leftarrow)$ Suponhamos que $X$ não é H.I, então existem $Y$ e $Z$ subespaços fechados de dimensão infinita de $X$ tal que $Y \cap Z=\{0\}$ e $Y+Z$ é fechado em $X$. Então pela Proposição 5.11 temos que inf $\{\|y-z\|:\|y\|=\|z\|=1, y \in Y, z \in Z\}>0$, absurdo.

Agora já estamos em condições de provar o principal resultado envolvendo operadores em espaços complexos hereditariamente indecomponíveis. Mostraremos que eles se escrevem de uma maneira simples e como Corolário teremos a demonstração de que esse espaço é um contra-exemplo para o Problema do Hiperplano.

Teorema 5.13 Se $X$ é um espaço de Banach complexo hereditariamente indecomponivel então todo $T \in L(X)$ pode ser escrito como $T=\lambda I+S$, onde $\lambda \in \mathbb{C}$ e $S$ é um operador estritamente singular.

\section{Demonstração:}

Suponhamos que existam $\lambda_{1}$ e $\lambda_{2}$ infinitamente singular para $T \operatorname{com} \lambda_{2}=\lambda_{1}+\beta, \beta \neq$ 0. 
Então $T-\lambda_{i} I$ não é um isomorfismo quando restrito a um subespaço de codimensão finita de $X$.

Se $Y_{0}=K \operatorname{er}\left(T-\lambda_{i} I\right)$ é de dimensão finita, então $X=Y_{0} \oplus Y$, onde $Y$ é um subespaço de $X$ de codimensão finita. Para $T-\left.\lambda_{i} I\right|_{Y}$ temos

1) $T-\left.\lambda_{i} I\right|_{Y}$ é linear e contínua.

2) $T-\left.\lambda_{i} I\right|_{Y}$ é injetor, pois $\operatorname{Ker}\left(T-\left.\lambda_{i} I\right|_{Y}\right)=\{0\}$.

3) $T-\left.\lambda_{i} I\right|_{Y}$ é sobre a imagem.

Então pelo Teorema da Aplicação Aberta $T-\left.\lambda_{i} I\right|_{Y}$ é um isomorfismo sobre a imagem. Absurdo, logo $Y_{0}$ tem dimensão infinita.

Consideremos $M=\operatorname{Ker}\left(T-\lambda_{1} I\right)$ e $N=\operatorname{K} \operatorname{er}\left(T-\lambda_{2} I\right)$. $M$ e $N$ são subespaços fechados de dimensão infinita.

1) $M \cap N=\{0\}$, pois suponha que exista $0 \neq x \in M \cap N$ então $T(x)=\lambda_{1} x$ e $T(x)=\lambda_{2} x, \operatorname{logo} \lambda_{1}=\lambda_{2} ;$ absurdo.

2) Vamos calcular $\|m-n\|$. Sejam $n \in N,\|n\|=1$ e $m \in M,\|m\|=1$. Temos $T(m)=\lambda_{1} m$ e $T(n)=\lambda_{2} n$.

$\|T\|\|m-n\| \geq\|T(m-n)\|=\|T(m)-T(n)\|=\left\|\lambda_{1} m-\lambda_{2} n\right\|=\left\|\lambda_{1} m-\lambda_{1} n-\beta n\right\|=$ $\left\|-\left(\lambda_{1} m-\lambda_{1} n-\beta n\right)\right\|=\left\|\beta n-\left(\lambda_{1} m-\lambda_{1} n\right)\right\| \geq\|\beta n\|-\left\|\lambda_{1}(m-n)\right\|=|\beta|\|n\|-$ $\left|\lambda_{1}\right|\|m-n\|$.

Com isto temos $\|T\|\|m-n\| \geq|\beta|\|n\|-\left|\lambda_{1}\right|\|m-n\|$ então $\|T\|\|m-n\|+\left|\lambda_{1}\right|\|m-n\| \geq$ $|\beta|$, logo $\|m-n\| \geq \frac{|\beta|}{\left(|T||+| \lambda_{1} \mid\right)}>0$.

Então pela Proposição $5.11 M+N$ é fechado em $X$. Tomando $W=M+N$ temos que $W$ é fechado e $W=M \oplus N$, absurdo pois $X$ é H.I. Portanto só pode existir no máximo um $\lambda$ infinitamente singular para $T$, então seja $\lambda$ infinitamente singular para $T$.

Suponhamos agora que $U=T-\lambda I$ não é estritamente singular, logo existem $\delta>0$ e $M$ subespaço fechado de $X$ de dimensão infinita tal que $\delta\|x\| \leq\|U(x)\|$.

O Kernel de $U$ é de dimensão infinita, pois se o Kernel fosse de dimensão finita $T-\left.\lambda I\right|_{Y}$ seria um isomorfismo, onde $Y$ é um subespaço de $X$ de codimensão finita; absurdo já que $\lambda$ é infinitamente singular. Seja $N=$ Ker $U$ então temos 
1) $M \cap N=\{0\}$, pois $\left.U\right|_{M}$ é isomorfismo logo dos elementos que estão em $M$ apenas $0 \in K \operatorname{Ker} U$.

2) Sejam $x \in M,\|x\|=1$ e $y \in N,\|y\|=1$, então $\|U\|\|x-y\| \geq\|U(x-y)\|=$ $\|U(x)-U(y)\|=\|U(x)\| \geq \delta\|x\|=\delta>0$.

Logo pela Proposição 5.11 segue que $M+N$ é fechado em $X$, então tomando $W=$ $M+N$ temos que $W$ é fechado e $W=M \oplus N$; absurdo pois $X$ é H.I.

Portanto $T-\lambda I$ é estritamente singular, ou seja $T-\lambda I=S$ onde $S$ é estritamente singular, logo $T=S+\lambda I$.

Corolário 5.14 Se X é um espaço de Banach complexo hereditariamente indecomponível, então $X$ não é isomorfo a nenhum subespaço próprio fechado. Em particular $X$ não é isomorfo a nenhum hiperplano fechado.

\section{Demonstração:}

Seja $Y$ um subespaço fechado de $X^{\prime}$ e $T: X \rightarrow Y \subset X$ um operador qualquer.

Pelo Teorema 5.13 existe $\lambda \in \mathbb{C}$ e $S^{\prime}: X \rightarrow X$ estritamente singular tal que $T=\lambda I+S$.

Se $\lambda=0$, então $T$ não é um isomorfismo, pois $T=S$.

Se $\lambda \neq 0$, então $i(\lambda I)=0$ e pelo Teorema 0.26 temos que $i(T)=i(\lambda I+S)=i(\lambda I)=$ 0 . Se $T$ é injetor então $\beta(T)=0$, logo $T$ é sobrejetor, em particular $X=Y$. Portanto $Y$ não é um subespaço próprio de $X$.

A partir de agora passaremos a estudar os operadores sobre espaços hereditariamente indecomponíveis reais.

Para demonstrarmos que esses espaços também são contra-exemplos para o Problema do Hiperplano, veja Teorema 5.17, precisamos de dois Lemas que nos ajudarão a trabalhar com o complexificado do espaço real, veja [20] pág 81, que estaremos considerando.

Para os próximos resultados $\hat{X}$ indicará o complexificado do espaço de Banach real $X$, ou seja, $\hat{X}=\{(u, v): u, v \in X\}$. Lembremos que o produto de $(u, v) \in \hat{X}$ pelo número complexo $a+i b$ é dado por $(a+i b)(u, v)=(a u-b v, a v+b u)$ e a norma em $\hat{X}$ é dada por $\|(u, v)\|=\max \left\{\left(\|a u-b v\|^{2}+\|a v+b u\|^{2}\right)^{1 / 2}: a^{2}+b^{2}=1\right\}$. 
Lema 5.15 Seja $X$ um espaço de Banach real de dimensão infinita e $Y$ um subespaço de codimensão finita de $X$, então $\hat{Y}$ também é um subespaço de codimensão finita de $\hat{X}$.

\section{Demonstração:}

Como $Y$ é um subespaço de codimensão finita de $X$ podemos escrever $X=Y \oplus A$ onde $A$ é um subespaço de $X$ de dimensão finita. Verificaremos agora que $\hat{X}=\hat{Y} \oplus \hat{A}$.

Seja $\left(x_{1}, x_{2}\right) \in \hat{X}$ então $x_{1}=y_{1}+a_{1}$ e $x_{2}=y_{2}+a_{2}, y_{i} \in Y, a_{i} \in A$ onde $i=1,2$, . $\left(x_{1}, x_{2}\right)=\left(y_{1}+a_{1}, y_{2}+a_{2}\right)=\left(y_{1}, y_{2}\right)+\left(a_{1}, a_{2}\right)$.

Logo cada elemento de $\hat{X}$ é escrito como a soma de um elemento de $\hat{Y}$ mais um elemento de $\hat{A}$. Verificaremos agora que esta soma é direta.

1) $\hat{Y} \cap \hat{A}=\{0\}$.

De fato, seja $\left(x_{1}, x_{2}\right) \in \hat{Y} \cap \hat{A},\left(x_{1}, x_{2}\right) \neq(0,0)$. Logo $\left(x_{1}, x_{2}\right)=\left(y_{1}, y_{2}\right)=\left(a_{1}, a_{2}\right)$ o que implica que $y_{1}=a_{1}$ e $y_{2}=a_{2}$, com isto temos que $Y \cap A \neq\{0\}$, absurdo.

2) $\hat{A}$ tem dimensão finita.

De fato, seja $\left\{\epsilon_{1}, \ldots, e_{n}\right\}$ base real de $A$, logo $\left\{\left(e_{1}, 0\right),\left(0, \epsilon_{1}\right),\left(e_{2}, 0\right),\left(0, e_{2}\right), \ldots,\left(e_{n}, 0\right)\right.$, $\left.\left(0, e_{n}\right)\right\}$ é base para $\hat{A}$, pois consideremos $(a, b) \in \hat{A}$ então $(a, b)=\left(\sum_{i=1}^{n} \alpha_{i} e_{i}, \sum_{i=1}^{n} \beta_{i} e_{i}\right)$, com $\alpha_{i}, \beta_{i} \in \mathbb{R}$.

$\left(\alpha_{1} e_{1}+\alpha_{2} e_{2}+\ldots+\alpha_{n} e_{n}, \beta_{1} e_{1}+\beta_{2} e_{2}+\ldots+\beta_{n} e_{n}\right)=\left(\alpha_{1} e_{1}, \beta_{1} e_{1}\right)+\ldots+\left(\alpha_{n} e_{n}, \beta_{n} e_{n}\right)=$ $=\alpha_{1}\left(e_{1}, 0\right)+\beta_{1}\left(0, \epsilon_{1}\right)+\ldots+\alpha_{n}\left(e_{n}, 0\right)+\beta_{n}\left(0, e_{n}\right)$.

Portanto $\hat{A}$ tem dimensão finita.

Por 1) e 2) temos que $\hat{X}=\hat{Y} \oplus \hat{A}, \hat{A}$ de dimensão finita e $\hat{Y}$ codimensão finita.

Lema 5.16 Seja $V: X \rightarrow X$ onde $X$ é um espaço de Banach real de dimensão infinita e considere $U: \hat{X} \rightarrow \hat{X}$ tal que $U(x, y)=(V(x), V(y))$. Se existe $Y \subset X$ de codimensão finita, tal que $\left.V\right|_{Y}$ é isomorfismo sobre a imagem então $\left.U\right|_{\hat{Y}}$ também é isomorfismo sobre a imagem.

\section{Demonstração:}

Por hipótese, existe $k \in \mathbb{R}$ tal que $k\|y\| \leq\|V(y)\|, \forall y \in Y$. Calculemos $U(x, y)$, $(x, y) \in \hat{Y} . U(x, y)=(V(x), V(y)), \log \circ$ 
$\|U(x, y)\| \geq \max \{\|(V(x)\|\| V,(y))\|\} \geq \max \{k\|x\|, k\|y\|\} \geq k \max \{\|x\|,\|y\|\} \geq$ $\frac{k}{2 \sqrt{2}}\|(x, y)\|$. Portanto $\left.U\right|_{\hat{Y}}$ é isomorfismo sobre a imagem.

Teorema 5.17 Seja $X$ um espaço de Banach real hereditariamente indecomponível, então $X$ não é isomorfo a nenhum de seus subespaços próprios.

\section{Demonstração:}

Consideremos $T \in L(X)$ e denotemos por $S$ a extensão natural de $T$ para a complexificação de $X$, ou seja, $S(x, y)=(T(x), T(y))$

Pelo Lema 5.9 existe $\lambda$ tal que $\lambda \notin F_{S}$, então $\lambda$ é infinitamente singular para $S$, ou seja, $S-\left.\lambda I\right|_{Z}$ nào é isomorfismo sobre a imagem, onde $Z$ é um subespaço de codimensão finita de $\hat{X}$.

caso $1: \lambda=0$.

Afirmamos que $\left.T\right|_{Y}$ não é isomorfismo sobre a imagem para todo subespaço $Y$ de codimensão finita de $X$. Pois se existisse $Y \subset X, Y$ de codimensão finita tal que $\left.T\right|_{Y}$ fosse um isomorfismo sobre a imagem então teríamos pelos Lemas 5.15 e 5.16 que $\hat{Y}$ seria de codimensão finita e $\left.S\right|_{\hat{Y}}$ seria um isomorfismo sobre a imagem, absurdo pois $\lambda$ é infinitamente singular para $S$. Verificaremos agora que $T-\lambda I=T$ é estritamente singular.

De fato, suponhamos que $T$ não é estritamente singular, logo existem $\delta>0$ e $M$ subespaço fechado de $X$ de dimensão infinita tal que $\delta\|x\| \leq\|T(x)\|$.

O Kernel de $T$ é de dimensão infinita, pois se o Kernel de $T$ fosse de dimensão finita $\left.T\right|_{Y}$ seria um isomorfismo, $Y$ de codimensão finita, absurdo. Seja $N$ o Kernel de $T$, temos

a) $M \cap N=\{0\}$.

De fato, $\left.T\right|_{M}$ é um isomorfismo, logo dos elementos que estão em $M$ apenas $0 \in \operatorname{KerT}$.

b) $M+N$ é fechado em $X$.

De fato, sejam $x \in M$ e $y \in N,\|x\|=\|y\|=1$ então $\|T\|\|x-y\| \geq\|T(x-y)\|=\|T(x)-T(y)\|=\|T(x)\| \geq \delta\|x\|=\delta>0$.

Portanto $M+N$ é fechado em $X$ pela Proposição 5.11. Então tomando $W=M+N$ 
temos que $W$ é fechado e $W=M \oplus N$, absurdo, pois $X$ é H.I.

Portanto $T$ é estritamente singular, logo $T$ não é um isomorfismo.

caso $2: \lambda \neq 0$.

Consideremos $T_{\lambda}=T^{2}-2 \operatorname{Re} \lambda T+|\lambda|^{2} I$. Então $(S-\bar{\lambda} I)(S-\lambda I)=\left(T_{\lambda}(x), T_{\lambda}(y)\right)$.

De fato, seja $\lambda=a+i b$, temos que $S(x, y)=(T(x), T(y))$.

$(S-\bar{\lambda} I)(S-\lambda I)=(T(T(x)-a x+b y), T(T(y)-a y-b x))-(a[T(x)-a x+b y]+b[T(y)-$ $a y-b x], a[T(y)-a y-b x]-b[T(x)-a x+b y])=\left(T^{2}(x)-2 a T(x)+a^{2} x+b^{2} x, T^{2}(y)-\right.$ $\left.2 a T(y)+a^{2} y+b^{2} y\right)=\left(T_{\lambda}(x), T_{\lambda}(y)\right)$.

Como $\lambda \notin F_{S}$ temos que $\forall \epsilon>0$ existe $Z_{\epsilon}$ subespaço fechado de dimensão infinita de $\hat{X}$ tal que $\|S-\lambda I\| \leq \frac{\epsilon}{\|S-\bar{\lambda} I\|}$ sobre $Z_{\epsilon}$, logo $\|(S-\bar{\lambda} I)(S-\lambda I)\| \leq\|(S-\bar{\lambda} I)\|\|(S-\lambda I)\| \leq \epsilon$ sobre $Z_{c}$.

Segue do Lema 5.3 que $\left.(S-\bar{\lambda} I)(S-\lambda I)\right|_{W}$ não é isomorfismo sobre qualquer subespaço $W$ de codimensão finita de $\hat{X}$.

Seja $L=(S-\bar{\lambda} I)(S-\lambda I): \hat{X} \rightarrow \hat{X}$ definida por $L(x, y)=\left(T_{\lambda}(x), T_{\lambda}(y)\right)$.

Análogo ao que vimos anteriormente temos que $\left.T_{\lambda}\right|_{Y}$ não é um isomorfismo sobre qualquer subespaço $Y, Y \subset X$ de codimensão finita. Novamente pelo Lema 5.3 temos que $\forall \epsilon>0$, existe $Y_{\epsilon}$ subespaço fechado de $X$ de dimensão infinita tal que $\left\|T_{\lambda}\right\| \leq \epsilon$ sobre $Y_{\epsilon}$. Verificaremos agora que $T_{\lambda}$ é estritamente singular.

Suponhamos que $T_{\lambda}$ não é estritamente singular, então existe $Y$ subespaço fechado de dimensão infinita de $X$ e $\delta>0$ tal que $\delta\|y\| \leq\left\|T_{\lambda}(y)\right\|, \forall y \in Y$. Tomemos $\epsilon<\delta$ e consideremos $Y$ e $Y_{\epsilon}$.

a) $Y \cap Y_{\epsilon}=\{0\}$.

De fato, pois se $0 \neq x \in Y \cap Y_{\epsilon}$, tomemos $\|x\|=1$, então $\delta\|x\| \leq\left\|T_{\lambda}(x)\right\|<\epsilon \operatorname{logo}$ $\delta \leq\left\|T_{\lambda}(x)\right\|<\epsilon$ portanto $\delta<\epsilon$, absurdo.

b) $Y+Y_{\epsilon}$ é fechado.

De fato, seja $y \in Y,\|y\|=1$ e $x \in Y_{\epsilon},\|x\|=1$.

$\left\|T_{\lambda}\right\|\|(y-x)\| \geq\left\|T_{\lambda}(y)-T_{\lambda}(x)\right\| \geq\left\|T_{\lambda}(y)\right\|-\left\|T_{\lambda}(x)\right\| \geq \delta-\epsilon>0$. 
Portanto $\|y-x\|>0$, logo pela Proposição $5.11 Y+Y_{\epsilon}$ é fechado.

Então tomando $W=Y+Y_{\epsilon}$ temos que $W$ é fechado e $W=Y \oplus Y_{\epsilon}$, absurdo $X$ é H.I. Portanto $T_{\lambda}$ é estritamente singular.

Verificaremos agora que $T$ é Fredholm com índice zero. Seja $K=T^{2}-2 \operatorname{Re} \lambda T+|\lambda|^{2} I$.

Afirmação: Escrevendo $T^{2}-2 \operatorname{Re} \lambda T+|\lambda|^{2} I=(T-a I)(T-b I)$, onde $a, b \in \mathbb{C}$ temos que $T-z$ é Fredholm para todo $z \neq a$ e $z \neq b$.

De fato, seja $v \in \mathbb{C}, v \neq a$ e $v \neq b$. Escolhamos $w \in \mathbb{C}$, tal que $v+w=2 R e \lambda$.

Logo $v w \neq|\lambda|^{2}$, pois caso contrário $v$ seria raiz de $z^{2}-2 R e \lambda z+|\lambda|^{2}=0$ e portanto seria igual a $a$ ou $b$.

Como $(T-v I)(T-w I)=T^{2}-(v+w) T+v w I=T^{2}-2 \operatorname{Re} \lambda T+v w I=K-\left(|\lambda|^{2}-\right.$ $v w) I$ e $K$ é estritamente singular e ainda $|\lambda|^{2}-v w \neq 0$, temos pelo Teorema 0.26 que $(T-v I)(T-w I)$ é Fredholm.

Consequentemente $T-v I$ também é Fredholm, pois $\operatorname{Ker}(T-v I) \subset K \operatorname{ker}((T-w I)(T-$ $v I))$ e $\operatorname{Im}((T-v I)(T-w I)) \subset \operatorname{Im}(T-v I)$.

Observemos que $a \neq 0$ e $b \neq 0$, pois $a$ e $b$ são raízes de $z^{2}-2 \operatorname{Re} \lambda z+|\lambda|^{2}=0$ e $\lambda \neq 0$.

Resta mostrarmos que o índice de $T$ é zero.

Vamos definir $\varphi: \mathbb{C} \backslash\{a, b\} \rightarrow \mathbb{Z}$, pondo $\varphi(z)=i(T-z I)$. $\varphi$ é contínua.

De fato, seja $z_{0} \in \mathbb{C}$, pelo Teorema 0.25 existe $\mu=\mu\left(T-z_{0} I\right)$ tal que se $P \in L(X)$ e $\|P\|<\mu$ então $i\left(P+T-z_{0} I\right)=i\left(T-z_{0} I\right)$.

Logo se $z \in \mathbb{C}$ é tal que $\left\|z-z_{0}\right\|<\mu$, então $\left\|\left(-z+z_{0}\right) I\right\|<\mu$, logo $i(T-z I)=$ $i\left(T-z_{0} I\right)$ e portanto $\varphi$ é contínua.

Mas $\mathbb{C} \backslash\{a, b\}$ é conexo, portanto $\varphi$ é constante. No entanto sabemos que se $|z|>\|T\|$ então $-z I+T$ é inversível, isto é, $i(T-z I)=0$, consequentemente $\varphi$ é nula, em particular $\varphi(0)=i(T)=0$.

Consequentemente se $T \in L(X)$ e é injetor então $\beta(T)=0$, isto é a imagem de $T$ é todo $X$ e portanto $X$ não pode ser isomorfo a nenhum de seus subepaço próprios.

Terminamos a dissertação observando ainda que não se sabe se o Teorema 5.13 é 
verdadeiro para espaços de Banach reais, isto é:

Problema 5.18 Seja X um espaço de Banach real hereditariamente indecomponível. É verdade que todo $T \in L(X)$ pode ser escrito na forma $T=\lambda I+S$, onde $\lambda \in \mathbb{R}$ e S é um operador estritamente singular? 


\section{Referências Bibliográficas}

[1] B. Beauzamy, Introduction to Banach Spaces and their Geometry, North-Holland, New York 1982.

[2] C. Bessaga and A. Pelczynski, A generalization of results R. C. James concerning absolute bases in Banach spaces, Studia Math., 17, 1958, 165-174.

[3] P. G. Casazza and T. J. Shura, Tsirelson's space, Lecture Notes in Mathematics, 1363.

[4] J. Diestel, Sequences and Series in Banach Spaces, Springer-Verlag, New York 1984.

[5] N. Dunford and J. Schwartz, Linear operator, vol 1. New York Interscience, 1958.

[6] E. M. Galego, Alguns aspectos de Geometria de espaços de Banach atraves de $c_{0}(\mathbb{N})$, IME-USP 1996.

[7] S. Goldberg, Unbounded Linear Operators, Theory and Aplications, Mc Graw-Hill Book Company.

[8] W. T. Gowers, Banach spaces not contaning $c_{0}, l_{1}$ or reflexive subspace, Trans. Amer. Math. Soc. 344, 1994, 407-420.

[9] W. T. Gowers, A solution to Banach's hyperplane problem, Bull. London Math. Soc. 26, 1994, 523-530.

[10] W. T. Gowers, A solution to the Schroeder-Bernstein problem for Banach space, Bull. London Math. Soc. 28, 1996, 297-304.

[11] W. T. Gowers, The unconditional basic sequence problem. Journal of the Americam Mathematical Society, vol. 6, número 4, outubro de 1993. 851-874. 
[12] W. T. Gowers, Banach spaces with small spaces of operator, Math. Ann. 307, (1997) 543-568.

[13] W. T. Gowers, A new dichotomy for Banach spaces, Geom. Funct. Anal. 6 (1996), número 6,1083-1093.

[14] R. B. Holmes, Geometric Functional Analysis and its applications, Springer-Verlag, New York 1975.

[15] C. S. Honig, Análise Funcional e Aplicações, vol 1, IME-USP 1970.

[16] R. C. James, Bases and reflexivity of Banach spaces, Ann. of Math. 52, 1950, 518-527.

[17] R. C. James, Uniformly non-square Banach spaces, Ann. of Math. 80 (1964), 542-550.

[18] R. C. James, Bases in Banach spaces, Amer. Math. Monthly 89 (1982), 9, 625-640.

[19] Elon Lages Lima, Curso de análise,vol. 2, R.J, Inst. de Matemática Pura e Aplicada, 1981.

[20] J. Lindenstrauss and L. Tzafriri, Classical Banach spaces, vol 1, Springer-Verlag, New York 1977.

[21] H. P. Rosenthal, The unconditional basic sequence problem. Contemp. Math. 52, AMS, 1986.

[22] Thomas Schlumprecht, An arbitrarily distortable Banach space, Israel Journal of Mathematics 76 (1991), 81-95.

[23] M. Spivak, Cálculo Infinitesimal, Editorial Reverté, S. A, 1970.

[24] A. E. Taylor, Introduction to functional analysis, second edition, New York Chichester Brisbane Toronto, 1979. 